\title{
Beam Diagnosis and Lattice Modeling of the Fermilab Booster
}

\author{
Xiaobiao Huang
}

Submitted to the faculty of the University Graduate School in partial fulfillment of the requirements

for the degree

Doctor of Philosophy

in the Department of Physics,

Indiana University

September 2005 
Accepted by the Graduate Faculty, Indiana Univeristy, in partial fulfillment of the requirement for the degree of Doctor of Philosophy.

Shyh-Yuan Lee, Ph.D.

John Beggs, Ph.D.

Doctoral

Committee

Michael W. Snow, Ph.D.

Rex Tayloe, Ph.D.

Eric Prebys, Ph.D.

August 29th, 2005 
Copyright (C)2005 by

Xiaobiao Huang

ALL RIGHTS RESERVED 


\section{ACKNOWLEDGEMENTS}

First of all, I wish to express my gratitude to my advisor, Dr. S. Y. Lee, for his persistent guidance over the course of this research effort. The physical insights that Dr. Lee shared with me have been invaluable to my study. His encouragement has been a driving force that constantly pushes me forward. I am also sincerely grateful to Eric Prebys, who, as the head of the Proton Source Department of Fermilab, along with Dr. Lee, has created the opportunity for me to participate the research for the Booster. Eric provided original orbit response data and BPM turn-by-turn data and stimulated further interests which lead to this research topic. His support ensured me to have necessary equipment and beam time for experiment. He also helped me getting initial experimental experience on the Booster. The completion of this research effort is made possible through the help from many colleagues at Fermilab. Raymond Tomlin helped me to understand the operation of the Booster and many technical details of its various components. Ray helped me to learn how to prepare the machine for experiments and in many cases set up the experiment and took data with me. Charles Ankenbrandt often provided thoughtful comments on my studies. His suggestions were critical for solving the orbit response problem in this study. Jim Lackey and Milorad Popvic proivded valuable input and help in various situations. Help from William Pellico and Todd Sullivan in the experiments were important. Jean-Francois Ostiguy offered valuable discussions on the singular value decomposition and other issues. Discussions with K. Y. Ng were important. Alexander Drozhdin provided valuable help on the original lattice model. James Amundson provided important help for the analysis of ion profile monitor data with his code. Discussions with Linda and Panagiotis Spentzouris were beneficial. I especially thank Sho Ohnuma for his continuing concern and interests in my research on the Booster. His comments 
and suggestions are thought-provoking and are often very helpful. The Booster staff are appreciated for their assistance in experiments. My office mate, Robert Zwaska, enlightened me through our discussions in many situations. My friend, Dr. Weiming Guo deserves special thanks for always being accessible for advices on various issues. The discussions with him on independent component analysis and the turn-by-turn data on APS he supplied to me have been important. Many other people offered their generous help in numerous occasions. I apologize for not being able to thank them all by names.

The support of my family in the years is essential to any success I can possibly have. My parents' expectations on me have led me overcome many difficulties along the way to today. My wife, Suyan Ling, has given up so much of herself just to support me with love and care. The importance of her support cannot be exaggerated. It is to her and our newborn baby, Julie, that I dedicate this thesis.

This work is supported by grants from DE-AC02-76CH03000, DOE DE-FG0292ER40747 and NSF PHY-0244793. 


\title{
ABSTRACT
}

\author{
Xiaobiao Huang
}

\section{Beam Diagnosis and Lattice Modeling of the Fermilab Booster}

A realistic lattice model is a fundamental basis for the operation of a synchrotron. In this study various beam-based measurements, including orbit response matrix (ORM) and BPM turn-by-turn data are used to verify and calibrate the lattice model of the Fermilab Booster. In the ORM study, despite the strong correlation between the gradient parameters of adjacent magnets which prevents a full determination of the model parameters, an equivalent lattice model is obtained by imposing appropriate constraints. The fitted gradient errors of the focusing magnets are within the design tolerance and the results point to the orbit offsets in the sextupole field as the source of gradient errors.

A new method, the independent component analysis (ICA) is introduced to analyze multiple BPM turn-by-turn data taken simultaneously around a synchrotron. This method makes use of the redundancy of the data and the time correlation of the source signals to isolate various components, such as betatron motion and synchrotron motion, from raw BPM data. By extracting clean coherent betatron motion from noisy data and separates out the betatron normal modes when there is linear coupling, the ICA method provides a convenient means to measure the beta functions and betatron phase advances. It also separates synchrotron motion from the BPM samples for dispersion function measurement. The ICA method has the capability to separate other perturbation signals and is robust over the contamination of bad BPMs. The application of the ICA method to the Booster has enabled the measurement of the linear lattice functions which are used to verify the existing lattice 
model. The transverse impedance and chromaticity are measured from turn-by-turn data using high precision tune measurements. Synchrotron motion is also observed in the BPM data.

The emittance growth of the Booster is also studied by data taken with ion profile monitor (IPM). Sources of emittance growth are examined and an approach to cure the space charge induced emittance growth for low energy synchrotron beams is discussed. 


\section{Contents}

Acceptance $\quad$ ii

$\begin{array}{lll}\text { Acknowledgements } & \text { v }\end{array}$

Abstract vii

1 The Booster Synchrotron 1

1.1 Overview of the Booster . . . . . . . . . . . . . . . 2

1.2 Motivation of Study . . . . . . . . . . . . . . . . . . . . . 12

2 Introduction to Accelerator Physics 18

2.1 Coordinate System . . . . . . . . . . . . . . . . . . 19

2.2 Basic Components of an Accelerator . . . . . . . . . . . . 20

2.2.1 Dipole ........................ 20

2.2.2 Quadrupole and skew quadrupole . . . . . . . . . . 21

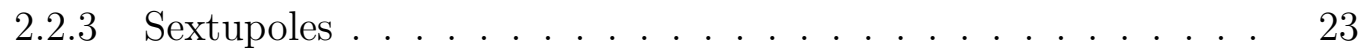

2.2 .4 RF cavity ...................... 23

2.3 Transverse Motion . . . . . . . . . . . . . . . . . . . 24

2.3.1 Equation of motion ............... 25

2.3.2 Transfer matrix . . . . . . . . . . . . . . . . . 27

2.3.3 Emittances ..................... 28 


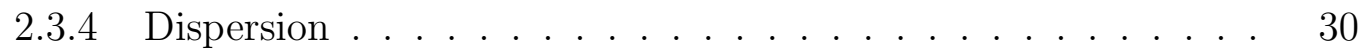

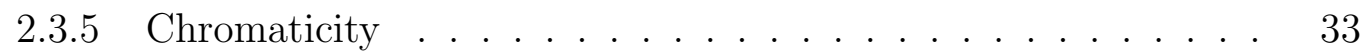

2.4 Longitudinal Motion . . . . . . . . . . . . . . . . . . 33

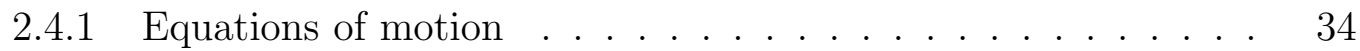

2.4.2 RF phase stability and $\mathrm{rf}$ bucket . . . . . . . . . . 35

2.4.3 Longitudinal emittance . . . . . . . . . . . . . . . . . . 38

2.4.4 Transition crossing ............... 40

3 Application of Orbit Response Measurements at Fermilab Booster 43

3.1 Introduction . . . . . . . . . . . . . . . . . . . . . 43

3.2 The ORM Fitting Model and Algorithm . . . . . . . . . . . . 46

3.2.1 The unconstrained fitting scheme . . . . . . . . . . . 46

3.2.2 Simulation with the PSR model . . . . . . . . . . . 50

3.2.3 Simulations with the Booster model . . . . . . . . . . . . . 51

3.3 The Constrained ORM Fitting Scheme . . . . . . . . . . . . . 56

3.4 Application to Experimental Data . . . . . . . . . . . . . . 59

3.4.1 Measurements of ORM . . . . . . . . . . 59

3.4.2 The fitting results . . . . . . . . . . . . . . . 61

3.5 Summary ............................ 70

4 Independent Component Analysis for BPM Data Analysis $\quad 72$

4.1 The Basics of PCA-based MIA . . . . . . . . . . . . . . . 74

4.2 ICA for Synchrotron Beam Diagnosis . . . . . . . . . . . . . 76

4.2.1 Principles of independent component analysis . . . . . . . . 77

4.2.2 The time-correlation based ICA and its application to BPM data 79

4.3 Simulation Studies . . . . . . . . . . . . . . 86

4.3.1 The simulation model with linear coupling . . . . . . . 86 
4.3.2 Application to tracking data . . . . . . . . . . . 96

4.4 Lattice Modeling with Measured Lattice Functions _ . . . . . . . . . 99

5 Application of ICA to the Fermilab Booster 102

5.1 Linear lattice function measurements . . . . . . . . . . . . . . . . . . 104

5.1.1 Data taken in DC mode . . . . . . . . . . . . . . . . . 104

5.1.2 Data taken in AC mode . . . . . . . . . . . . . . 105

5.2 Measurements of Transverse Impedance . . . . . . . . . . . . . . . . . 114

5.3 Chromaticity Measurements . . . . . . . . . . . . . 126

5.4 Observation of Synchrotron Motion . . . . . . . . . . . . 133

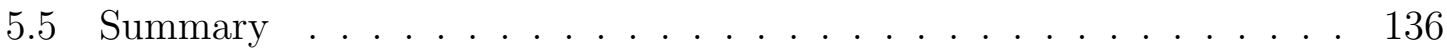

6 Emittance Growth $\quad 140$

6.1 The Emittance Measurements . . . . . . . . . . . . . . . . . . . . 141

6.2 The Vertical Emittance . . . . . . . . . . . . . . . . . 145

6.2.1 Linear growth region . . . . . . . . . . . . . . . . . 147

6.2.2 Space charge dominated region . . . . . . . . . . . . . 149

6.3 Horizontal Emittance . . . . . . . . . . . . . . . . . 154

6.3.1 Below transition energy . . . . . . . . . . . . . 155

6.3 .2 Across the transition energy . . . . . . . . . . . . 161

6.3.3 Microwave instability . . . . . . . . . . . . . . . . . . 162

6.3.4 Bunch mismatch oscillations in the synchrotron phase space . 162

6.3.5 Above transition energy . . . . . . . . . . . . . 165

6.4 Emittance Dilution and Other Observations . . . . . . . . . . . . 171

6.4.1 Effect of rf cavities at non-zero dispersive locations . . . . . . 171

6.4.2 Other observations .................. 173

6.5 Space Charge Half-integer Stopband Correction . . . . . . . . . . . . 173 
6.5.1 Envelope oscillation and half-integer stopband . . . . . . . . . 174

6.5.2 Stopband correction . . . . . . . . . . . . . . . . 175

6.5.3 Stopband correction for the Booster . . . . . . . . . . . . 178

6.6 Summary . . . . . . . . . . . . . . . . . . . . 180

7 Conclusions and Discussions 183

$\begin{array}{ll}\text { A Booster kinematic parameters } & 189\end{array}$ 


\section{List of Tables}

1.1 Maximum beta functions and dispersion function $\ldots \ldots \ldots$

1.2 The basic Booster parameters . . . . . . . . . . . . . . . . 13

3.1 The $\chi^{2}$ contributions in ORM fitting . . . . . . . . . . . 62

3.2 The ORM data frames and corresponding $\chi^{2} \ldots \ldots \ldots$

5.1 Chromaticity measurement in DC cycles . . . . . . . . . . 133

6.1 The concerned harmonics before and after correction $\ldots \ldots \ldots$

A.1 Booster kinematic parameters . . . . . . . . . . . . . . . . . 192

A.2 Booster kinematic parameters - continued . . . . . . . . . . 193 


\section{List of Figures}

1.1 Layout of the Booster tunnel. . . . . . . . . . . . . . . 2

1.2 Schematics of the combined-function magnets . . . . . . . . . . 4

1.3 The Booster injection scheme . . . . . . . . . . . . . . 8

1.4 The Booster extraction scheme . . . . . . . . . . . . . 8

1.5 The beta and dispersion functions of an ideal Booster cell. . . . . . . 9

1.6 Beam loss before and after 2003 fall shutdown . . . . . . . . . . . 15

2.1 The coordinate system. . . . . . . . . . . . . . . . . . . . . . . 19

2.2 Schematic of a dipole magnet. . . . . . . . . . . . . . . . . . 21

2.3 Schematics of quadrupole magnets . . . . . . . . . . . . . . . . 22

2.4 The Poicare section. . . . . . . . . . . . . . . . 37

2.5 The rf phase stability condition. . . . . . . . . . . . . . 37

2.6 Synchrotron Hamiltonian tori and separatrix . . . . . . . . . . . . . . 39

2.7 Matched and mismatched beam . . . . . . . . . . . . . . . 41

$3.1 \chi^{2}$ history for ORM simulation with PSR model . . . . . . . . . 52

3.2 Comparisons of solutions to simulation parameters for PSR . . . . . 53

3.3 Simulation fitting result with single model parameter error . . . . . . 54

3.4 The correlation coefficients for model parameters of neighboring magnets 55

$3.5 \sqrt{\chi^{2}}$ vs. norm of model parameters . . . . . . . . . . 57 
3.6 Examples of constrained parameter combinations _ . . . . . . . 60

3.7 Comparison of dispersion functions before and after the fitting . . . . 63

3.8 Model and measured orbit response for trim HS1 before and after fitting 64

3.9 The fitted $\frac{\Delta p}{p}$ and orbit response at section L20 $\ldots \ldots \ldots \ldots$

3.10 The fitted BPM and kicker gains . . . . . . . . . . 67

3.11 Constrained parameter combinations in the fitting result . . . . . 68

3.12 Linear correlation of the gradient errors and horizontal orbit offsets . 71

4.1 Mode separation ability of ICA and PCA with linear coupling . . . . 89

4.2 Mode separation ability of ICA and PCA with colored bad BPM signal 92

4.3 Mode separation ability of ICA and PCA with noisy bad BPM . . . . 93

4.4 Errors of measured $\beta$ and $\psi$ with random noise $\ldots \ldots \ldots \ldots$

4.5 Effect of finite number of turns _ . . . . . . . . . . . . 95

4.6 Verifying that ICA betatron modes are normal modes . . . . . . . . 98

4.7 Nonlinear resonance signal in tracking data . . . . . . . . . . . . . . . 99

5.1 Temporal and spatial patterns of DC data $\ldots \ldots \ldots \ldots$

5.2 FFT spectra of the temporal patterns . . . . . . . . . . . . 107

5.3 Horizontal beta function and phase advance . . . . . . . . . . 108

5.4 The injection momentum mismatch and the dispersion function . . . 109

5.5 Temporal patterns of betatron modes and the FFT spectra of AC data 110

5.6 Spatial patterns of betatron modes for AC data . . . . . . . . . . 111

5.7 Beta functions measured with $\mathrm{AC}$ data . . . . . . . . . . . . . 112

5.8 Horizontal and vertical betatron tunes in the Booster cycle . . . . . . 113

5.9 Temporal patterns of the betatron modes of AC data with two repositioned doglegs . . . . . . . . . . . . . . . . . 115

5.10 Spatial patterns of betatron modes of AC data with two re-positioned

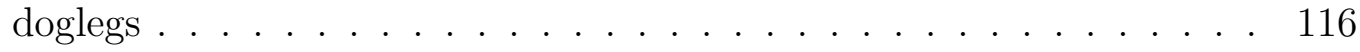


5.11 Beta functions and phase advances with two re-positioned doglegs . . 117

5.12 Vertical betatron tunes before transition for various intensity levels . 118

5.13 Typical betatron modes before and after transition . . . . . . . . . 119

5.14 Fitting $\Delta \nu_{z}$ vs. intensity to linear curves . . . . . . . . . . . . . . . . 121

5.15 The fitted zero-charge betatron tune and tune-intensity dependence . 122

5.16 The measured and calculated bunching factor . . . . . . . . . . . 123

5.17 The measured imaginary transverse impedance . . . . . . . . . . . . 124

5.18 The betatron tunes of the chromaticity measurement . . . . . . . . 128

5.19 The measured chromaticity . . . . . . . . . . . . . . . . 129

5.20 Chromaticities calculated with the model . . . . . . . . . . . . 130

5.21 Currents of chromaticity sextupoles . . . . . . . . . . . . . 131

5.22 Horizontal chromaticity reduction due to SEXTS sextupoles . . . . . 131

5.23 The measured chromaticity with both SEXTL, SEXTS off . . . . . . 132

5.24 Examples of synchrotron modes in Booster ramping cycle . . . . . . . 135

5.25 Synchrotron tune measured from the turn-by-turn BPM data . . . . . 136

5.26 The variation of momentum deviation for 50 turns . . . . . . . . . 137

5.27 The synchrotron spatial patterns normalized by dispersion function . 138

6.1 Total charge for cycles with different injection turns . . . . . . . . . . 142

6.2 The space charge perveance parameter for an entire cycle . . . . . . . 143

6.3 Beta and dispersion functions at the IPM in the Booster cycle . . . . 144

6.4 Comparison of the raw and filtered $\sigma_{z}^{2} \ldots \ldots$. . . . . . . . . 145

6.5 The properties of the digital filter . . . . . . . . . . . . . 146

6.6 Vertical emittance in the cycle for two intensity levels . . . . . . . . . 146

6.7 Linear growth region of vertical emittance. . . . . . . . . . . . . . 148

6.8 The vertical emittance growth rate before and after transition . . . . 149

6.9 Vertical emittance growth in the early cycle . . . . . . . . . . . 150 
6.10 Fitting the early vertical emittance growth to model . . . . . . . . . 152

6.11 The fitted parameters of the emittance growth model . . . . . . . . . 153

$6.12 \sigma_{x}^{2}$ for 4 -turn and 12 -turn injection $\ldots \ldots \ldots \ldots \ldots \ldots$

6.13 The rf voltage $V$ (RFSUM) and the synchronous phase $\phi_{s} \ldots \ldots$.

6.14 The rf bucket area, rms momentum width and rms bunch length . . . 156

6.15 Scaling laws of horizontal emittance and momentum spread . . . . . . 157

6.16 Fitting curves for the separation of horizontal emittance and momentum spread contributions . . . . . . . . . . . . . . . . 158

6.17 Fitting results for the separation of horizontal emittance and momentum spread contributions . . . . . . . . . . . . . . 160

6.18 The bunch length and momentum spread measured with resistive wall

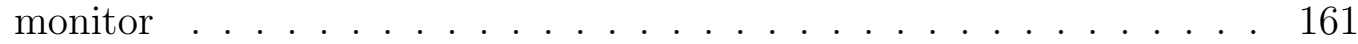

6.19 The effective space charge voltage $V_{\text {spchg }} \ldots \ldots \ldots \ldots \ldots$

6.20 Example of post-transition $\sigma_{x}^{2}$ fitting $\ldots \ldots \ldots \ldots \ldots$

6.21 The post-transition $\sigma_{x}^{2}$ fitting parameters $\ldots \ldots \ldots \ldots$

6.22 The post-transition $\sigma_{x}^{2}$ fitting parameters - continued . . . . . 167

6.23 The maximum and minimum rms momentum width $\delta_{1}$ and $\delta_{2}$ calculated from the oscillation amplitude of $\sigma_{x}^{2}$. . . . . . . . 168

6.24 The average rms momentum width $\bar{\delta}$ obtained with two methods . . . 170

6.25 The coherent betatron motion of the beam excited by the energy gain in $\mathrm{rf}$ cavities . . . . . . . . . . . . . . . . . . . . . . 172

6.26 Comparison of $\Delta \phi_{x}$ of MAD calculation to harmonic expansion . . 176

6.27 Envelop stopband integral before and after correction . . . . . . . . 179

A.1 Ramping parameters of turn number, $\beta, E_{k}$ and $P \ldots \ldots \ldots$.

A.2 Ramping parameters of $f_{\mathrm{rf}}, \Delta E_{k}, \eta$ and $T_{0} \ldots \ldots \ldots \ldots$ 


\section{Chapter 1}

\section{The Booster Synchrotron}

The Fermilab Booster is a fast cycling proton synchrotron. It is the first synchrotron in the Fermilab accelerator chain. The Booster takes proton beams from the Linac at a kinetic energy of $400 \mathrm{MeV}$ and accelerates them to $8 \mathrm{GeV}$ in $33.5 \mathrm{~ms}$. The beams are then extracted, to be transferred to the Main Injector for further acceleration or sent to the target of the MiniBooNE experiment. The Booster was built over 35 years ago and was first operated in 1970. It had a major upgrade in 1993 with the injection energy increased to the current $400 \mathrm{MeV}$ from the original $200 \mathrm{MeV}$. It is able to deliver $5 \times 10^{1} 2$ protons per pulse at $5 \mathrm{~Hz}$ repetition rate. As the Booster is the common source of high energy proton beams at Fermilab, its performance is crucial for all major Fermilab experiments. This chapter will first give an overview of the Booster in section 1.1 which includes the general aspects of the Booster, its lattice structure, correction elements and diagnostics. Then a brief description of the motivation of this study is given in section 1.2. 


\subsection{Overview of the Booster}

The Booster is composed of 24 identical cells with a total circumference of $474.2 \mathrm{~m}$. The cells are labeled $1,2, \cdots, 24$ along the direction of the beam with cell 1 contains the injection section. The layout of the Booster ring is shown in Fig. 1.1. Each

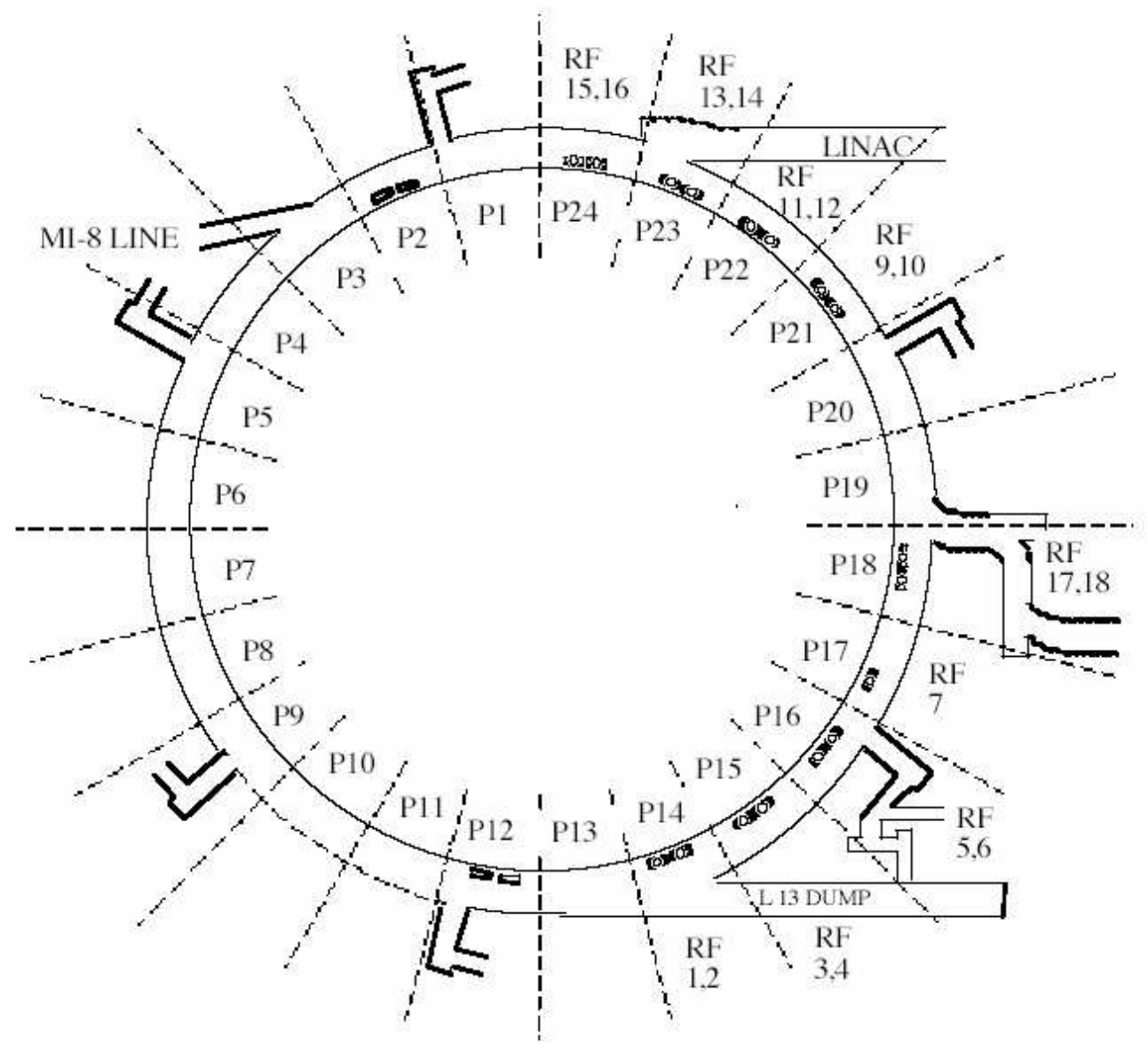

Figure 1.1: Layout of the Booster tunnel.

cell has 4 main magnets. The 96 main magnets bend the beam to form a circular path. Unlike most modern synchrotrons, the Booster magnets are combined-function magnets which serve as both dipole magnets and quadrupole magnets, i.e., they also 
provide transverse focusing of the beam. There are two types of main magnets, focusing (F magnets, see Fig. 1.2(a)) and defocusing (D magnets, see Fig. 1.2(b)) ${ }^{1}$. There are two F magnets and two D magnets in one cell which are configured as shown in Fig. 1.2(c), where FU, FD are focusing magnets and DU, DD are defocusing magnets, respectively. The second letter "U" or "D" indicate upstream or downstream. The straight section between the two defocusing magnets is $6 \mathrm{~m}$ long and is referred as a Long straight section. The space between the two neighboring focusing magnets is $1.2 \mathrm{~m}$ and is called a Short straight section. The long straight sections are used to install injection/extraction magnets, rf cavities, correction magnets and diagnostics, etc. Correction magnets and diagnostics are also installed in short straight sections.

The magnetic field in the magnets should ramp up together with the beam momentum to confine the beam around the fixed radial orbit within the vacuum pipe. The bending dipole field is of the form

$$
B(t)=B_{\mathrm{dc}}-B_{\mathrm{ac}} \cos 2 \pi f t, \quad f=15 \mathrm{~Hz}
$$

with $t=0$ at injection and $t=1 / 30 \mathrm{~s}$ at extraction. Because the radial orbit of the beam is fixed in the pipe on average, the beam momentum has to follow the magnetic field. Thus Eq. (1.1) can be used to predict the momentum in the cycle. A magnetic field of the form of Eq. (1.1) is generated by the power supply system through a resonant circuit. Each pair of F and D magnets are connected with a capacitor bank and a choke to form a resonance cell. There are a total of 48 cells which are connected in series and are powered by four power supplies. The resonance circuit oscillates at $15 \mathrm{~Hz}$ with sinusoidal current of the form of Eq. (1.1).

The beam gains energy from the electric field in the gaps of rf cavities during

\footnotetext{
${ }^{1}$ Since a horizontal focusing magnet is defocusing in the vertical plane and vice versa, one needs to specify in which plane the magnet is focusing. It is conventional to refer to the horizontal plane by default.
} 

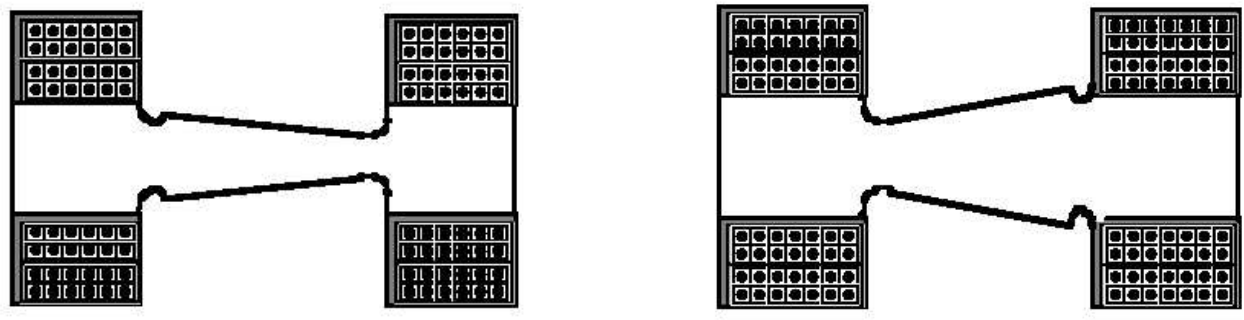

(a) focusing

(b) defocusing

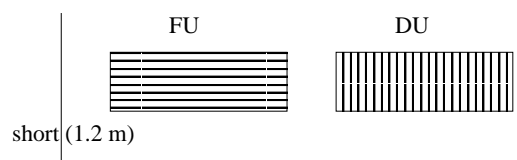

long (6 m)

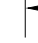

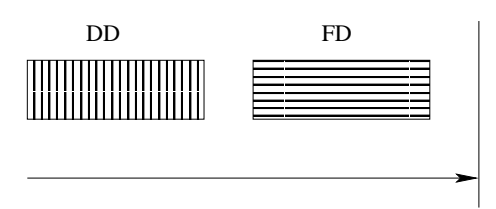

(c) cell layout

Figure 1.2: (a)(b) Cross sections of the combined-function magnets. (c) The layout of one Booster cell. The magnet length is $2.89 \mathrm{~m}$. 
acceleration. There are a total of $17 \mathrm{rf}$ cavities in the Booster. The cavities are installed in the long straight sections of 9 cells as shown in Fig. 1.1. The cavities are divided into two groups, group "A" and "B". The phase difference between the two groups determines the net acceleration voltage per turn. The rf frequency is 84 times the revolution frequency. It ranges from $37.87 \mathrm{MHz}$ at injection to $52.81 \mathrm{MHz}$ at extraction, corresponding to revolution time of $2.22 \mu \mathrm{s}$ and $1.59 \mu \mathrm{s}$, respectively. The ramping rf frequency is specified by a program which is stored in an electronics card. The rf frequency is changed by varying the current of the ferrite tuners. Two feedback loops, the phase lock loop (PLL) and the radial position loop, are used to make corrections to the program to keep the beam in correct positions and damp synchrotron oscillations. The radial position loop uses a BPM at Long 18 to detect the radial position (RPOS) of the beam ${ }^{2}$. The radial position is compared to the radial offset curve $(\mathrm{ROF})$. The difference signal is used to change the acceleration voltage and maintain the desired radial position. The PLL measures the phase error between the beam and the rf through a resistive wall monitor. The phase error signal is processed to generate corrections which then are applied to the rf cavities. The synchrotron oscillation is damped by accelerating or decelerating the beam.

The rf cavities not only accelerate the particles but also provide a longitudinal focusing force to hold them together to form a bunch structure. There are 84 stable phase space areas, or rf buckets that can capture and hold particles. Hence there are typically 84 bunches inside the ring. The entire sequence of bunches in one cycle is called a batch. The bunched particles occupy certain areas in phase space which is called the longitudinal emittance. The typical longitudinal emittance for the Booster is $0.1 \mathrm{eV} \cdot \mathrm{s}$. There is a critical point in the cycle called the transition when particles with different energies complete a revolution with the same time. At transition the

\footnotetext{
${ }^{2}$ After the shutdown in fall 2004, RPOS is picked up in Long 20.
} 
rf cavities lose their focusing power temporarily. The rf phase needs to be quickly shifted to maintain longitudinal focusing after transition. For the Booster, transition occurs at $17 \mathrm{~ms}$ after injection when the relativistic gamma is 5.446 .

The Booster adopts a multi-turn $\mathrm{H}^{-}$stripping injection scheme. The particles from the Linac are negative hydrogen ions $\left(\mathrm{H}^{-}\right)$. They are transferred from the Linac to the Booster tunnel through the $400 \mathrm{MeV}$ transport line. The final section of the transfer line is a matching section which consists of several suitably-configured quadrupole magnets. These quadrupoles rotate the transverse phase space ellipse of the beam to match that of the Booster to increase injection efficiency. The Debuncher is also located in this section. The Debuncher rotates the beam in longitudinal phase space to reduce the momentum spread and to reduce the $805 \mathrm{MHz}$ rf structure of the Linac. A set of four magnets (Orbump, see Fig. 1.3) are used to merge the injection beam to the Booster orbit. The injection beam at the end of the $400 \mathrm{MeV}$ line is bent to a trajectory parallel to the Booster beam path and meets the circulating beam (protons) at the second Orbump magnet. Both the proton and $\mathrm{H}^{-}$beams then pass the stripping foil which removes the electrons of $\mathrm{H}^{-}$ions. The stripped $\mathrm{H}^{-}$ions become protons and are bent to Booster beam orbit by the downstream Orbump magnets. The Orbump magnets are powered on only during injection which can last up to $40 \mu \mathrm{s}$, or 18 turns. The regular operations often inject 10 turns. Fig. 1.3 illustrates the injection scheme of the Booster.

The beam is extracted to the MI- 8 line at Long 03 to be transferred to the Main Injector. It can also be extracted to the beam dump at Long 13 (see Fig. 1.1). The extraction schemes are the same at both locations. Take the Long 03 extraction as an example. There are four fast kickers in the long section before the extraction section, i.e., Long 02. The extraction kickers have a rise time of approximately 30 ns. When fired, they push the beam upward by about $25 \mathrm{~mm}$ at the extraction section such that 
the beam passes the field region of the septum ${ }^{3}$ which then bends the beam further up by $44 \mathrm{mrad}$ to the MI- 8 line. Because the vertical aperture of the F magnets is small ( $42 \mathrm{~mm}$ ) and the septum plate has to be close to the beam centerline $(10 \mathrm{~mm}$ above), the beam does not have much space to occupy during acceleration. A set of four magnets are used to bend the beam downward at the septum to avoid scraping (see Fig. 1.4). These magnets form the extraction chicane and are commonly referred to as "dogleg" magnets. The dogleg magnets are not ramped because the beam size shrinks as its energy increases. A set of three magnets work together with the dogleg magnets to maintain aperture.

The nominal horizontal and vertical betatron tunes are 6.7 and 6.8 , respectively. The design lattice has strict 24-fold periodicity. Fig. 1.5 shows the beta function and dispersion function of one ideal Booster cell. It was believed that the ideal lattice was a good approximation of the real Booster lattice until recently when a more realistic lattice model [5] was built. It is found that the edge-focusing of the dogleg magnets perturb the linear lattice functions much more than expected. For example, the maximum horizontal beta is $\max \left(\beta_{x}\right)=47.1 \mathrm{~m}$ with dogleg effect while the design lattice has only $\max \left(\beta_{x}\right)=33.7 \mathrm{~m}$. The maximum vertical beta function and dispersion function are also increased. Increased beta function and dispersion function leads to increased beam size which in turn increases the possibility of beam loss. The maximum horizontal beam size is about $25 \%$ larger and the maximum vertical beam size is about $10 \%$ larger than the ideal lattice due to the dogleg effect. The perturbation of doglegs also reduces the periodicity of the lattice structure and causes other problems. For example, it can increase the resonance stopband width.

Theoretically, the dogleg effect can be partially compensated by properly arranging the trim quadrupoles [6]. However, it is difficult to implement because the

\footnotetext{
${ }^{3}$ The septum is a pulsed magnet which has a field-free region and a field region separated by a plate.
} 


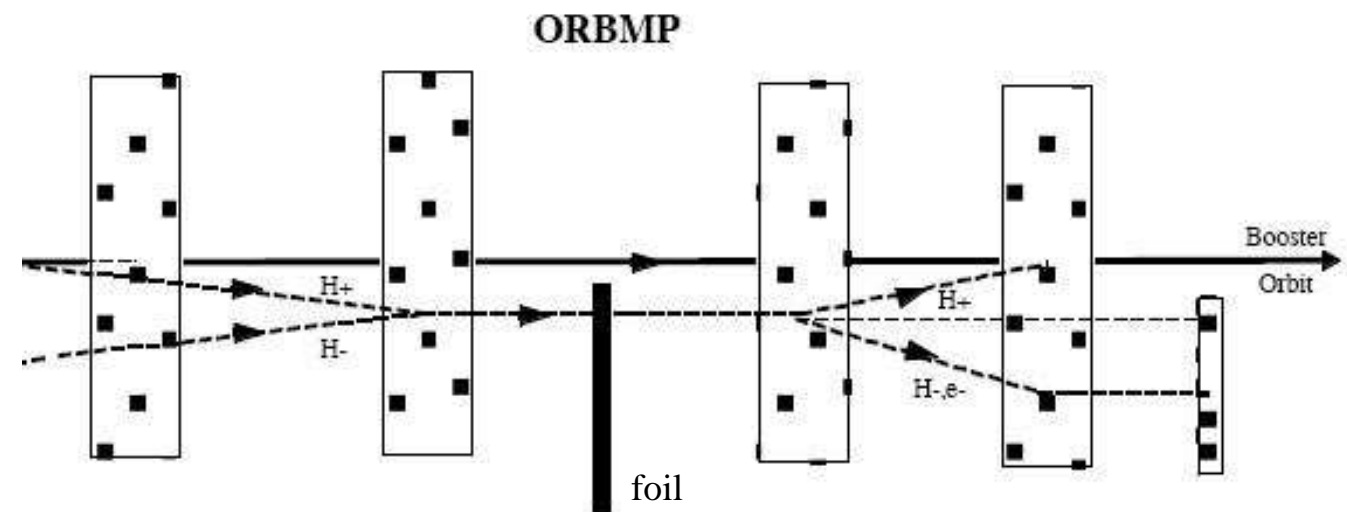

Figure 1.3: Schematic of the Booster injection scheme (from Ref.

$[3])$.

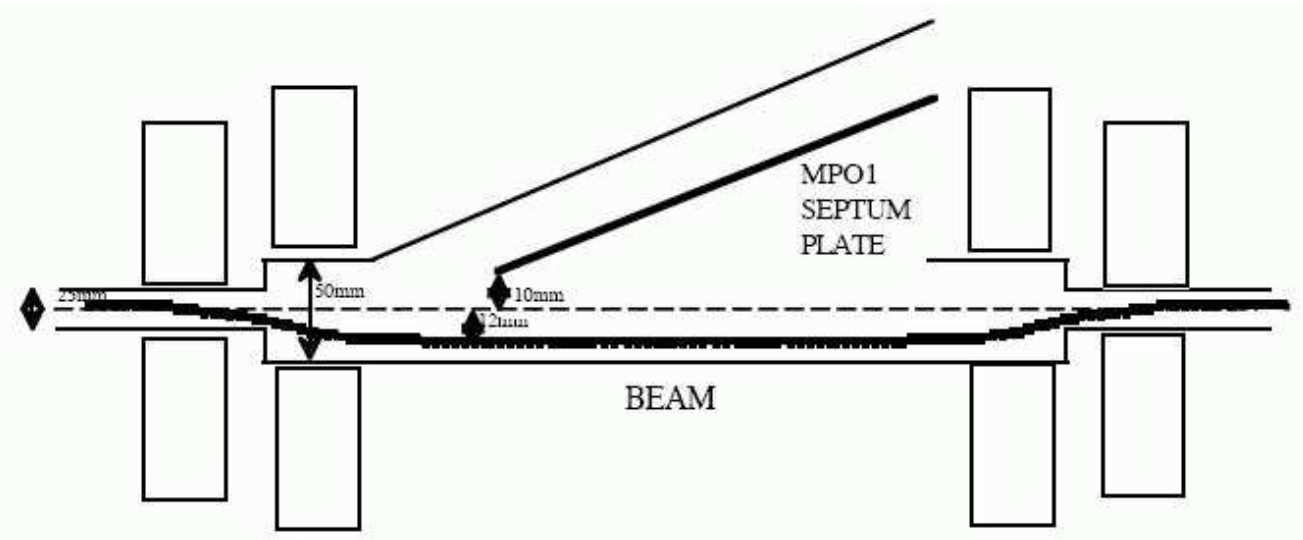

Figure 1.4: Schematic of the Booster extraction region (from Ref. $[3])$. 


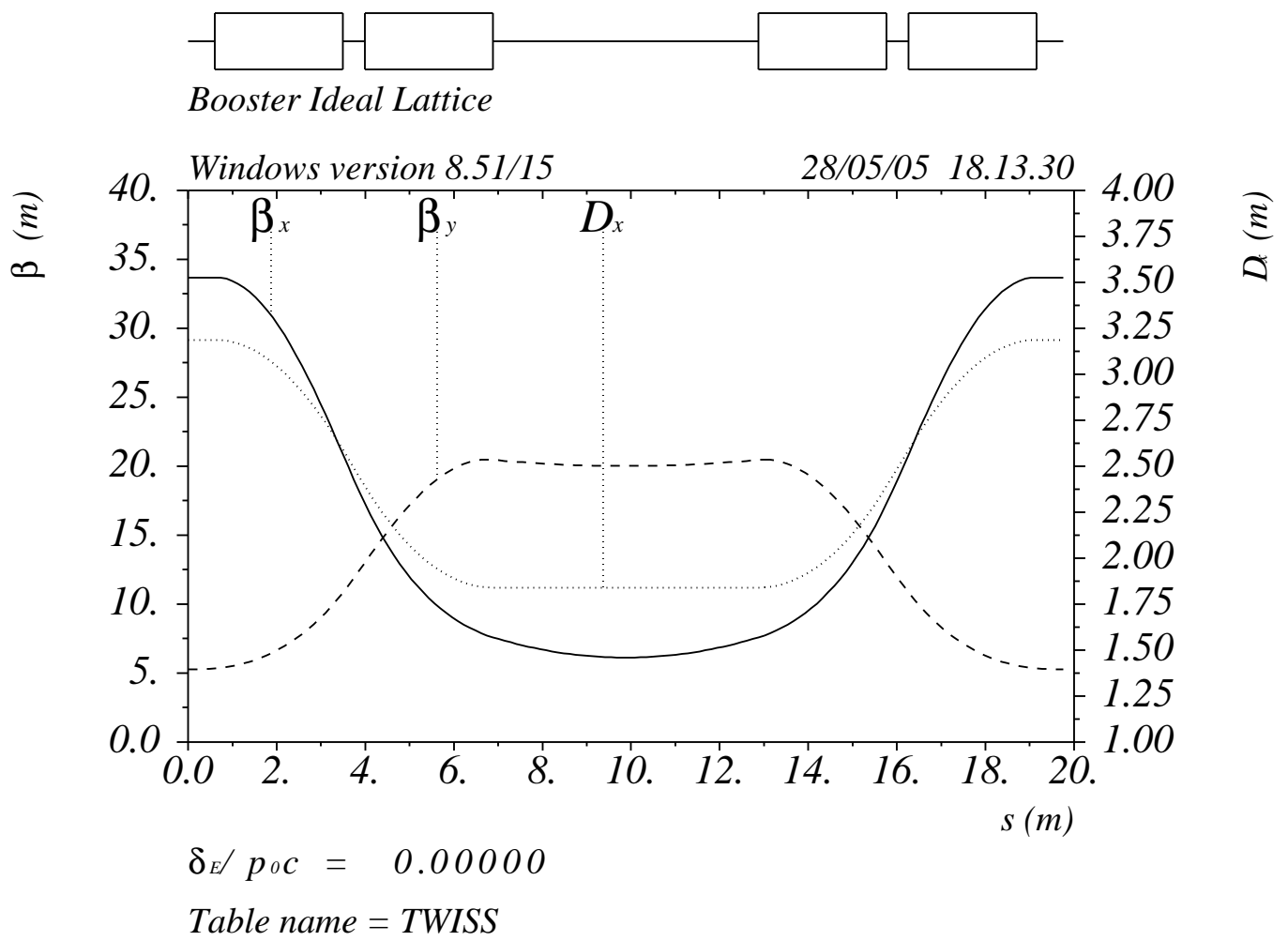

Figure 1.5: The beta and dispersion functions of an ideal Booster cell. 
applicability of the calculated compensation setting depends on the accuracy of the lattice model and the compensation result is not conveniently measurable. Even if the compensation scheme works, it reduces the flexibility of betatron tune control. An effective way to mitigate the dogleg effect is to reduce the bending angle $\theta$ of the dogleg magnet because the perturbation is proportional to $\theta^{2}$. For this reason, the two sets of dogleg magnets were repositioned in the 2003 fall shutdown and the 2004 fall shutdown, respectively. The distances between the two upstream (to the septum) and the two downstream dogleg magnets were both increased from original $0.21 \mathrm{~m}$ to $0.77 \mathrm{~m}$. With the new dogleg layout, the maximum horizontal beta function is $36.5 \mathrm{~m}$. The lattice periodicity is also restored. The maximum beta and dispersion function with different dogleg layout are listed in Table 1.1. The repositioning of dogleg magnets has resulted in sizeable improvements of injection efficiency. The lattice functions before and after the dogleg magnet repositioning have been measured through BPM (beam position monitor) turn-by-turn data in this study.

Table 1.1: The maxima of linear lattice functions

\begin{tabular}{||l|l|l|l||}
\hline dogleg layout & $\beta_{x}(\mathrm{~m})$ & $\beta_{z}(\mathrm{~m})$ & $D_{x}(\mathrm{~m})$ \\
\hline ideal (no doglegs) & 33.7 & 20.5 & 3.2 \\
old 03, old 13 & 47.1 & 24.2 & 6.1 \\
new 03, old 13 & 41.3 & 23.9 & 4.7 \\
new 03, new 13 & 36.5 & 23.6 & 3.7 \\
\hline
\end{tabular}

The 96 combined-function magnets form the main frame of the Booster lattice. However, a real machine is never perfect because of the inevitable manufacturing defects, installation errors and operation variations. It is necessary to have additional magnets which can be used to fine-tune the machine to improve performance. 
The Booster has various correction elements, including dipoles, quadrupoles, skew quadrupoles and sextupoles. There are a total of 48 corrector packages evenly distributed in the ring with one in each long straight section and each short straight section. A corrector package is a combination of a horizontal trim dipole, a vertical trim dipole, a trim quadrupole and a skew quadrupole. These elements are all independently controllable. The trim dipoles are used to control the beam orbits. Since one trim dipole causes orbit distortion globally, the trim dipoles are often used in combinations to create local orbit "bumps". The short section horizontal dipoles are most efficient for horizontal orbit control because the short sections correspond to large horizontal beta function. Similarly the long section vertical dipoles are most efficient for vertical orbit control. The trim dipoles are usually powered with DC current (i.e., not ramped). The trim quadrupoles are used to control the betatron tunes. The long section quadrupoles are better for vertical tune control and the short section quadrupoles are better for horizontal tune control. The trim quadrupoles are ramped. The long section trim quadrupoles share a ramping curve and the short section trim quadrupoles share another. A change of current by $1 \mathrm{~A}$ at $400 \mathrm{MeV}$ in a short section quadrupole would result in a horizontal tune shift of 0.020 and the same amount of change in a long section quadrupole would result in a vertical tune shift of 0.011 . The skew quadrupoles are used to compensate linear coupling between horizontal and vertical betatron motions. The Booster has chromaticity sextupoles in some short sections and some long sections to control the horizontal and vertical chromaticities. The short section chromaticity sextupoles is ramped with the SEXTS curve and the long section chromaticity sextupoles is ramped with the SEXTL curve. The Booster also has sextupoles in some of the long sections for third order resonance compensation.

The diagnostics of the Booster provides various means to monitor the beam. There is a beam position monitor (BPM) in each of the 48 straight sections. The BPM 
detector is composed of four striplines in a cylinder. The difference between signals from the pair of top-bottom striplines are used for vertical beam position detection and the left-right pair are for horizontal beam position. The horizontal and vertical beam positions are simultaneously measured at the same location. The positions can be digitized on a turn-by-turn basis. The Booster ion profile monitors (IPMs) are non-destructive tools for transverse beam profile measurements. They are located at long straight section 05 . The IPMs detect ions generated by the interaction between the beam and residual gas in the vacuum pipe to derive the transverse profile of the beam. Both horizontal and vertical IPM readings are recorded turn by turn. The transverse sizes are measured from the recorded profiles. Since the space charge force of the beam affects the motion of ions and thus the ion profile, the transverse beam sizes derived from the profiles need to be calibrated with respect to beam intensity [7]. A resistive wall monitor (RWM) is located at the Long 18 section to pick up the beam current signal. The signal from the RWM is used to measure the longitudinal bunch positions (phase) and profiles. About 60 beam loss monitors (BLM) are in the Booster tunnel to measure the proton beam loss.

The basic parameters of the Booster are listed in Table 1.2.

\subsection{Motivation of Study}

For some time, the primary proton use of the Booster was antiproton production for the Tevatron $p \bar{p}$ collision experiment. It requires about $7 \mathrm{E} 15$ protons per hour, a task that the Booster can handle easily. However, the Fermilab neutrino programs, namely, the MiniBooNE and NuMi/Minos experiments, have increased the proton demand significantly over the next few years. The proton demand was increased to as much as 1.8E17 protons per hour. Since the Booster is now able to accelerate 5E12 protons per pulse and the maximum repetition rate allowed by hardware is 7.5 
Table 1.2: The basic Booster parameters

\begin{tabular}{||l|l||}
\hline Circumference $(\mathrm{m})$ & 474.2 \\
Injection kinetic energy $(\mathrm{MeV})$ & 400 \\
Extraction kinetic energy $(\mathrm{GeV})$ & 8 \\
Cycling rate $(\mathrm{Hz})$ & 15 \\
RF frequency $(\mathrm{MHz})$ & $37.8-52.8$ \\
Harmonic number & 84 \\
Protons per cycle & $5 \times 10^{12}$ \\
Super-periodicity & 24 \\
Magnets per cell & 4 \\
Betatron tunes $\nu_{x}, \nu_{z}$ & $6.7,6.8$ \\
max/min $\beta_{x}(\mathrm{~m})$ & 33.7 (Short)/6.1 (Long) \\
max/min $\beta_{z}(\mathrm{~m})$ & 20.5 (long)/5.3 (Short) \\
max/min $D_{x}(\mathrm{~m})$ & 3.19 (Short)/1.84 (Long) \\
Transition $\gamma_{t}$ & 5.446 \\
Transverse emittance ${ }^{a} \epsilon_{n}(\mathrm{~mm}-\mathrm{mrad})$ & $12 \pi$ \\
Longitudinal emittance ${ }^{b}(\mathrm{eV} \cdot \mathrm{s})$ & 0.1 \\
\hline
\end{tabular}

${ }^{a}$ normalized $95 \%$ emittance

${ }^{b} 95 \%$ emittance 
$\mathrm{Hz}$, it seems not far from the goal. However, beam loss prevents the Booster from delivering its full production rate. Proton beam loss in the Booster causes potential radiation damage to the accelerator components and the radiation activation of the components makes the maintenance difficult. The beam loss is currently the main limiting factor of the Booster which allows an operational proton flux level of only $0.5 \mathrm{E} 17$ protons per hour. Thus the major challenge is to increase the proton output without increasing beam loss.

It is important to understand the causes of beam loss to be able to reduce it. The lattice can have a large impact over the performance of the machine, which can be seen from the improvement of injection efficiency of the Booster after the distance between the dogleg magnets was stretched out during the fall shutdown in 2003 (see Fig. 1.6). Although the Booster is more than 35 years old, our understanding of its lattice was not very good. For example, the beta functions had never been measured. The lattice distortion effect of the extraction doglegs was not discovered until recently because there was no effort to check the lattice model with experiment. Is the lattice model realistic after the discovery of dogleg effect? Are there any other large lattice distortion sources such as main magnet imperfections? We are not able to tell without verification of the model with beam-based measurements.

The first effort in this study is to calibrate the lattice model with orbit response matrix (ORM) measurements. Orbit responses are the beam orbit deviations when the beam is perturbed by trim dipoles. The orbit responses are determined entirely by the linear properties of the lattice. By comparing the measured orbit responses to that predicted by the lattice, the lattice model can be calibrated. The ORM method has been successfully applied to many electron storage rings. Even though it is somewhat challenging to apply it to a fast-cycling proton machine because of the reduced orbit stability and BPM resolution, it is still a promising approach to verify and correct the Booster lattice model. 

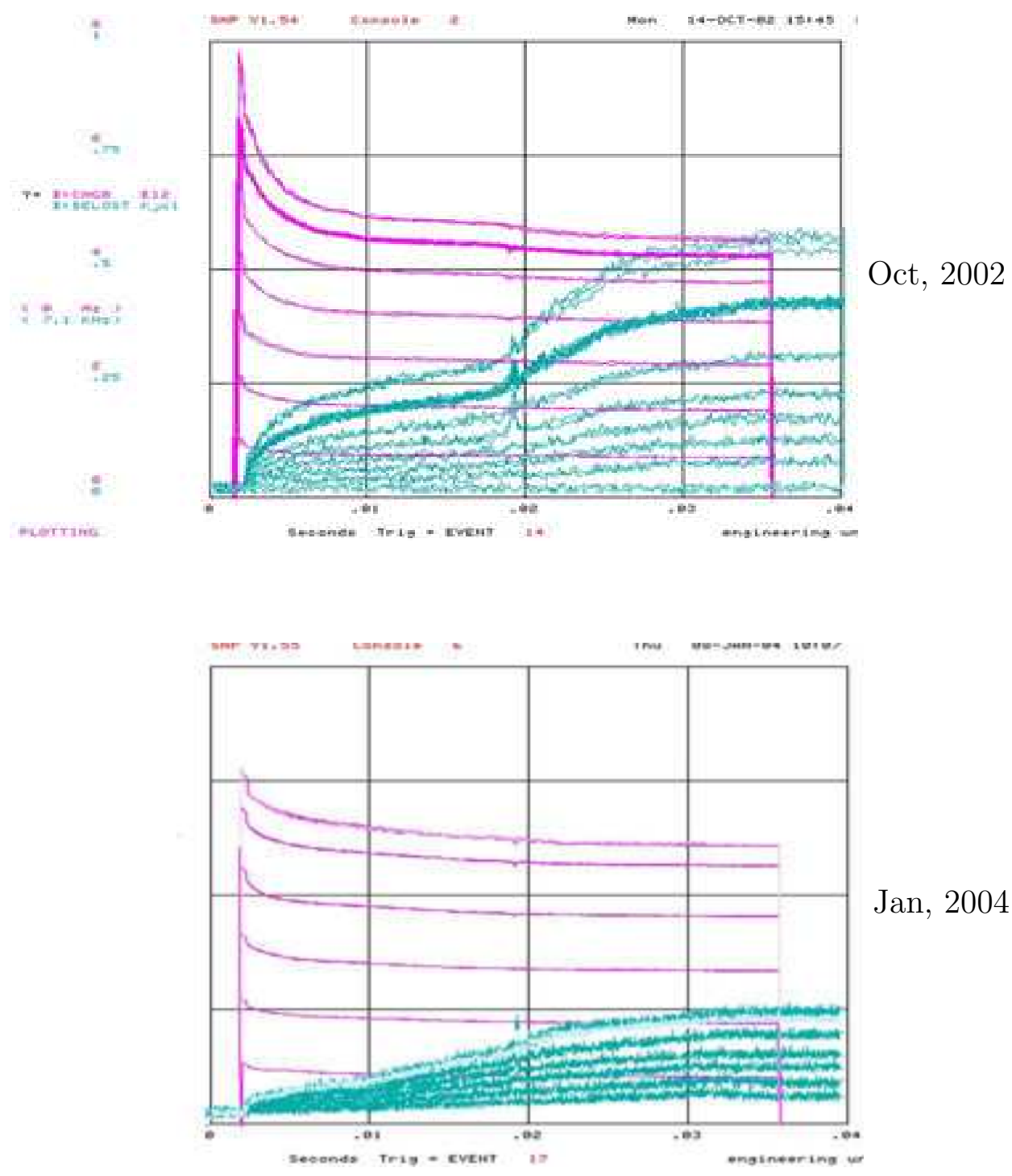

Jan, 2004

Figure 1.6: (Color) Typical beam intensity (magenta) and beam loss (green) before and after 2003 fall shutdown when the dogleg in L13 is stretched out. The two plots use the same scale. The intensity scale is 0 to $8 \times 10^{12}$ protons per pulse. The time axis (horizontal) corresponds to one cycle. (from Eric Prebys) 
The Booster BPMs have been upgraded to be able to measure the beam orbit on turn-by-turn basis for the entire cycle. The turn-by-turn BPM data contain a vast amount of information about the beam motion. The beta functions, betatron phase advances and dispersion function can be measured from the turn-by-turn data, which in turn can be used to verify and calibrate the lattice model. Betatron tunes can be measured from turn-by-turn data. By measuring the dependence of betatron tunes on relevant factors, other information such as the transverse impedance and the chromaticities can be derived. BPM turn-by-turn data also has information about the synchrotron motion. However, the turn-by-turn readings of each BPM have mixed information of the betatron and, synchrotron motions and are often contaminated by white or colored noise due to electronics system. It is difficult to study one component without being bothered by the others. The signal-to-noise ratio of single BPM readings is often not high because the amplitude of coherent motion of the beam is limited by beam loss. Thus it is important to take advantage of the data of all BPMs to uncover the information of the machine. As BPMs of more and more accelerators are able to read turn-by-turn data, the suitable data analysis method has become a compelling issue. In this study, the applicability of a new data analysis technique called the independent component analysis (ICA) is studied. This method has many desirable features for turn-by-turn data analysis and is expected to become a common beam diagnosis tool.

Emittance growth is also a concern in Booster operation. The early blowup of transverse emittance is a major cause of injection beam loss for high intensity beams in the Booster. The cause of emittance growth is believed to be the space charge effect. The pattern of emittance growth and its dependence on beam intensity is studied through the ion profile monitor (IPM) measurements. A possible mechanism of transverse emittance growth is the space charge stopband associated with the halfinteger envelope resonance. A cure of the space charge induced emittance growth and 
beam loss is proposed on the basis of this theory. Beam loss at transition is a major source of tunnel activation. It is also an important limiting factor of the maximum batch size (proton per pulse). The longitudinal non-adiabatic motion near transition and longitudinal space charge effect cause phase space mismatch which in turn lead to post-transition oscillation. This is observed and studied with both IPM and resistive wall monitor signal.

This thesis is organized into seven chapters. This first chapter describes the Fermilab Booster and the motivation of study. A concise introduction of the basics of accelerator physics is given in Chapter two. Chapter three presents the orbit response matrix measurements and model calibration. Chapter four introduces the method of independent component analysis (ICA) for BPM turn-by-turn data analysis. Application of the ICA method to the Booster is described in Chapter five. The study of emittance growth and a possible cure is found in Chapter six. Conclusions are summarized in Chapter seven. 


\section{Chapter 2}

\section{Introduction to Accelerator}

\section{Physics}

In a circular accelerator, particles are guided by the magnetic field of the bending magnets to follow the design orbit. Quadrupole magnets are also needed to prevent loss of particles due to divergence. Since a quadrupole magnetic field always provides a focusing force on one transverse direction and a defocusing force on the other transverse direction, modern accelerators adopt an alternating gradient focusing scheme which applies focusing and defocusing quadrupole magnets alternately to obtain an overall focusing effect on both transverse directions. Particles in a beam oscillate around the nominal orbit in the transverse directions.

Particles gain energy only at the gaps of rf cavities which have longitudinal electric field. The electric field oscillates at radio frequencies. Particles pick up energy at an rf gap only if their arrival time is in the right half rf period when the electric field favors the increase of energy, and the amount of energy each particle gains also depends on its arrival time. Usually the revolution time of particles depends on their energy. By properly setting the rf phase, particles with higher energy can be made to gain less 
energy from rf cavities and vice versa. Particles are held together to form bunches due to the longitudinal focusing effect of rf cavities. Particles in bunched beams oscillate in the longitudinal direction around the nominal energy and phase.

This chapter intends to introduce the basics of accelerator physics as a basis for the discussions in the following chapters. The coordinate system used in this study is introduced in section 2.1. A brief description of the basic components of a synchrotron is in section 2.2. The theory of transverse and longitudinal motions are given in section 2.3 and section 2.4 , respectively.

\subsection{Coordinate System}

In accelerator physics the orbits of particles are measured by their deviation from a reference orbit. The reference orbit is the ideal path of the beam determined by the location and magnetic fields of the deflecting magnets. It is a fixed, closed path. An ideal particle with right momentum and initial conditions would travel along the reference orbit. But in general particles oscillate around it.

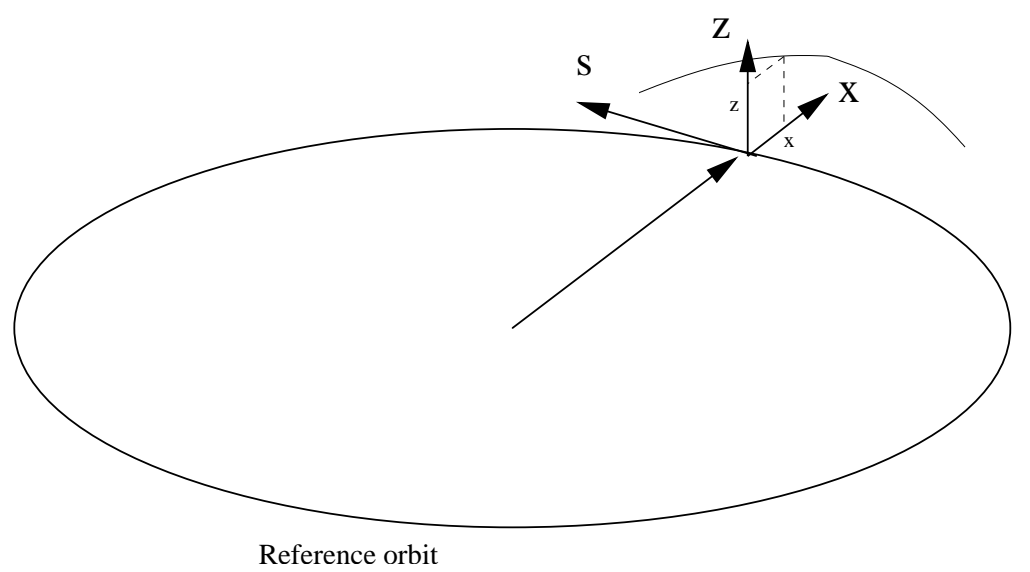

Figure 2.1: The coordinate system. 
The coordinate system used in this thesis is as shown in Fig. 2.1. The local Cartesian coordinate axis $(\mathbf{x}, \mathbf{s}, \mathbf{z})$ moves along the reference orbit $\mathbf{S}$ with $\mathbf{s}$ pointing to the tangent of $\mathbf{S}, \mathbf{x}$ in the tangential plane to the outer side and $\mathbf{z}=\mathbf{x} \times \mathbf{s}$. The $\mathbf{x}, \mathbf{z}$ direction are referred as horizontal and vertical directions, respectively.

The arc length $s$ traveled by the ideal particle along the reference orbit is often used as independent variable instead of time $t$. The relation of the two is $s=\beta c t$ with $\beta c$ the speed of the ideal particle. The transverse "velocity" is then

$$
x^{\prime}=\frac{\mathrm{dx}}{\mathrm{ds}}, \quad z^{\prime}=\frac{\mathrm{dz}}{\mathrm{ds}}
$$

which actually depicts the deflecting angle in the two transverse planes respectively.

\subsection{Basic Components of an Accelerator}

An accelelerator consists of various magnets such as dipole magnets, quadrupole and skew quadrupole magnets, sextupole and skew sextupole magnets, etc. RF cavities are also essential for acceleration and longitudinal manipulation. The properties of these components are summarized below.

\subsubsection{Dipole}

A dipole magnet generates uniform magnetic field which bends the beam running through it by a certain angle $\theta$. The dipole length $l$ and bending radius of curvature $\rho$ are related by $l=\rho \theta$. A schematic drawing of a dipole magnet is shown in Fig. 2.2. The bending radius is determined by both the magnetic field and the momentum of the beam. Let $B_{0}$ be the dipole field and $p$ the momentum of a particle with charge $e$, then

$$
\rho=\frac{p}{e} \frac{1}{B_{0}}
$$


where $p / e$ is defined as the momentum rigidity of the beam and is often written as $B \rho=p / e$.

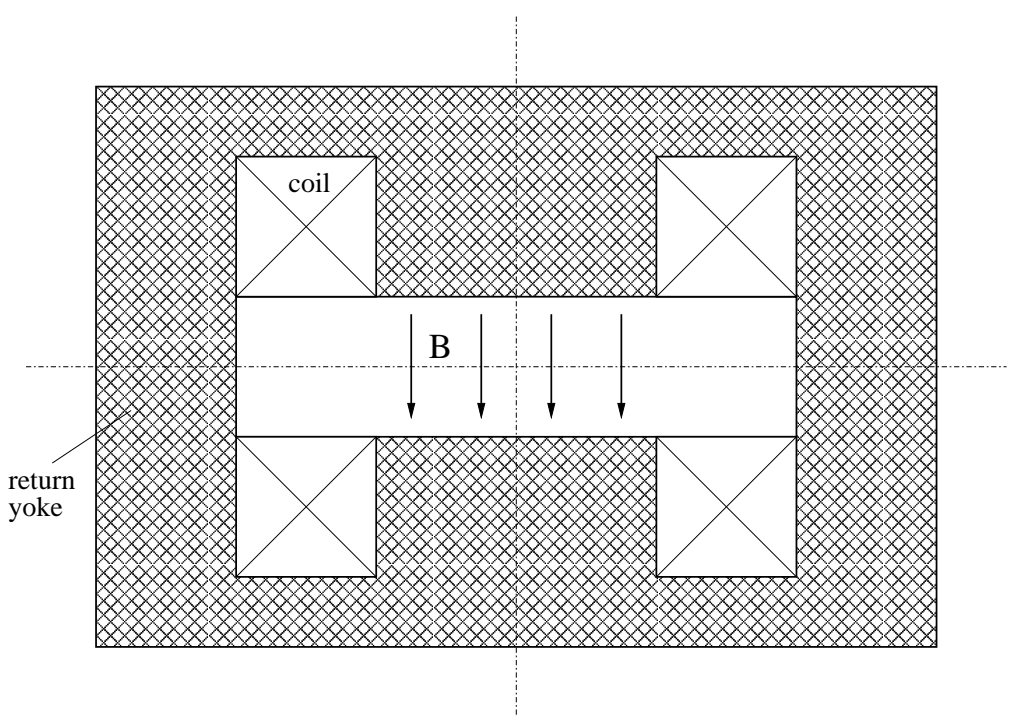

Figure 2.2: Schematic of a dipole magnet.

\subsubsection{Quadrupole and skew quadrupole}

Quadrupole magnets are used to generate a transverse focusing force to confine the beam in the vacuum pipe. Skew quadrupole magnets are used to correct the linear coupling of motions between the two transverse planes. Fig. 2.3 shows schematics of both magnet types.

In a quadrupole magnet the magnetic field depends on position linearly. The magnetic field gradient in the two transverse directions are equal by symmetry. The quadrupole coefficient is defined as

$$
B_{1}=\frac{\partial B_{x}}{\partial z}=\frac{\partial B_{z}}{\partial x}
$$




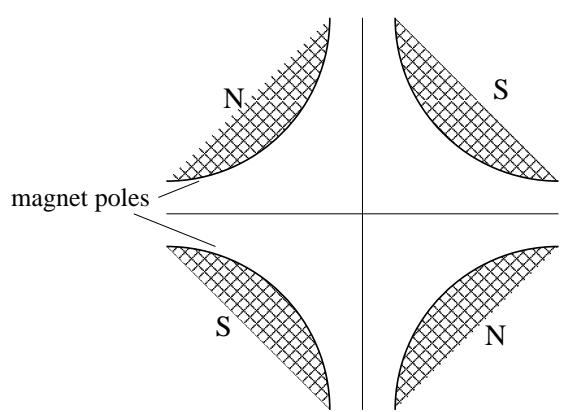

(a) quadrupole

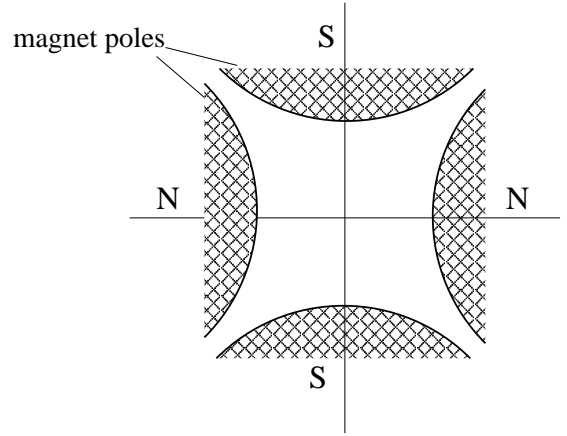

(b) skew quadrupole

Figure 2.3: Schematics of a normal quadrupole and a skew quadrupole.

and it is normalized by the momentum rigidity to give

$$
K_{1}=\frac{B_{1}}{B \rho}
$$

The reference orbit usually runs through the center of the quadrupole magnets. So the magnetic field inside a quadrupole magnet is

$$
\begin{aligned}
B_{x} & =B_{1} z, \\
B_{z} & =B_{1} x .
\end{aligned}
$$

A particle passing the quadrupole magnetic field receives an angular kick that depends on its transverse position. For a thin quadrupole magnet with length $l$, the angular kicks are

$$
\begin{aligned}
\Delta x^{\prime} & =\frac{\int B_{z} \mathrm{dl}}{B \rho}=K_{1} l x, \\
\Delta z^{\prime} & =-\frac{\int B_{x} \mathrm{dl}}{B \rho}=-K_{1} l z .
\end{aligned}
$$


So a focusing magnetic field for the horizontal plane is defocusing for the vertical plane and vice versa.

Skew quadrupoles are normal quadrupoles rotated 45 degrees about the $s$ axis. It relates the focusing force in one transverse plane with the position in the other transverse plane. The transverse motions in both planes are coupled by skew quadrupoles. They are usually used to compensate the linear coupling caused by skew quadrupole components due to magnet imperfections or misalignments.

\subsubsection{Sextupoles}

Sextupoles and skew sextupoles are nonlinear components because their magnetic field depend quadratically on transverse position. The magnetic field of a sextupole is

$$
\begin{aligned}
& B_{x}=B_{2} x z \\
& B_{z}=B_{2} \frac{\left(x^{2}-z^{2}\right)}{2},
\end{aligned}
$$

where the sextupole coefficient $B_{2}$ is defined as

$$
B_{2}=\frac{\partial^{2} B_{z}}{\partial x^{2}}
$$

and it is normalized by defining $K_{2}=B_{2} /[B \rho]$. Sextupoles provide position-dependent focusing/defocusing magnetic field. They are used to compensate the chromaticity as will be discussed in section 2.3. Skew sextupoles are obtained by rotating sextupoles about the $s$ axis by $30 \mathrm{deg}$.

\subsubsection{RF cavity}

RF cavities supply energy to accelerate the beam or compensate its energy loss and provide longitudinal focusing. An rf cavity affects the beam by establishing a longitudinal electric field across its gap. Charged particles passing the gap will gain or lose 
energy according to the arrival time. The electric field oscillates with radio frequency $f_{\text {rf }}$. The voltage across the gap is of the form

$$
V(t)=V_{0} \sin \left(\omega_{\mathrm{rf}} t+\phi\right)
$$

where $\omega_{\mathrm{rf}}=2 \pi f_{\mathrm{rf}}$ is angular frequency and $\phi$ is a phase factor. If the gap is narrow, the energy gain of a particle in one pass is $e V(t)$. For the beam to be synchronized with the rf cavity, the rf frequency $f_{\text {rf }}$ should be an integral multiple of the revolution frequency of the beam. This integer $h$ is called the harmonic number

$$
f_{\mathrm{rf}}=h f_{\mathrm{rev}} \text {. }
$$

\subsection{Transverse Motion}

Under the alternating focusing/defocusing force of gradient magnets, particles in a beam undergo oscillations around the reference orbit in the transverse directions. Such oscillation is called betatron motion which is to be discussed in this section. 


\subsubsection{Equation of motion}

Motion of charged particles in magnetic field is determined by the Lorentz force law. The equations of motion can be derived ${ }^{1}$ in our curvilinear coordinate system to get

$$
\begin{aligned}
x^{\prime \prime}-\frac{\rho+x}{\rho^{2}} & =\frac{B_{z}}{B \rho} \frac{p_{0}}{p}\left(1+\frac{x}{\rho}\right)^{2}, \\
z^{\prime \prime} & =-\frac{B_{x}}{B \rho} \frac{p_{0}}{p}\left(1+\frac{x}{\rho}\right)^{2},
\end{aligned}
$$

where $\rho$ is the local radius of curvature of the orbit and $p$ is momentum of the particle being described while $p_{0}$ is the momentum of the ideal particle which travels along the reference orbit and $B \rho=p_{0} / e$ is the momentum rigidity of this particle. Particles with momentum $p=p_{0}$ are said to be on-momentum. For on-momentum particles, if we consider only up to the linear term, i.e., the dipole and quadrupole fields, the above equations are reduced to

$$
\begin{aligned}
x^{\prime \prime}+K_{x}(s) x & =0, \quad K_{x}=1 / \rho^{2}-K_{1}(s), \\
z^{\prime \prime}+K_{z}(s) z & =0, \quad K_{z}=K_{1}(s) .
\end{aligned}
$$

Since modern accelerators are often composed of sequential identical sections (each section is called a superperiod), the focusing functions $K_{x}(s), K_{z}(s)$ are often periodic

\footnotetext{
${ }^{1}$ This can be done by starting with the general Hamiltonian of a charged particle in electromagnetic field [1]

$$
H=e \phi+\sqrt{E_{0}^{2}+|\mathbf{P}-e \mathbf{A}|^{2}}
$$

where $\phi$ is the electric potetial, $\mathbf{A}$ is the magnetic vector potential, $E_{0}$ and $\mathbf{P}$ are the inertial energy and the canonical momentum of the particle, respectively. Alternatively one may consider Newton's law under the Lorentz force

$$
\frac{\mathrm{d} \mathbf{p}}{\mathrm{dt}}=e(\mathbf{E}+\mathbf{v} \times \mathbf{B})
$$

with $\mathbf{p}=\mathbf{P}-e \mathbf{A}$ represents the mechanical momentum, $\mathbf{E}$ is the electric field and $\mathbf{B}$ is the magnetic field.
} 
(for circular accelerators, the circumference $C$ is a natural period). An equation of the form

$$
y^{\prime \prime}+K(s) y=0
$$

with periodic coefficient $K(s+L)=K(s)$ is called Hill's equation. Our discussion of betatron motion is based on Eq. (2.17) with $y$ represents either $x$ or $z$.

According to Floquet's theory, the general solutions of Hill's equation can be expressed in terms of two basis functions of the form

$$
y_{1}(s)=a w(s) \exp [i \psi(s)], \quad y_{2}(s)=a w(s) \exp [-i \psi(s)]
$$

with periodic condition that $w(s+L)=w(s)$. Hill's equation requires that

$$
\begin{gathered}
w^{\prime \prime}+K(s) w-\frac{1}{w^{3}}=0, \\
\psi^{\prime}=\frac{1}{w^{2}} .
\end{gathered}
$$

From Eq. (2.18) we see $w(s)$ is related to the oscillation amplitude. Eq. (2.19) is the envelope equation which is also difficult to solve. In general it may be solved numerically. Conventionally one defines the Courant-Snyder parameters as

$$
\beta=\frac{1}{w^{2}}, \quad \alpha=-w w^{\prime}, \quad \gamma=\left(1+\alpha^{2}\right) / \beta
$$

in which $\beta$ is called betatron amplitude function or beta function. From Eq. (2.20) we have

$$
\psi(s)=\int_{0}^{s} \frac{\mathrm{ds}}{\beta(s)}
$$

where $\psi(s)$ is the phase function. Eq. (2.22) implies that beta function is the local wave number of betatron oscillation. Both $\beta(s)$ and $\psi(s)$ are determined only by the 
placements and strengths of the magnets of the accelerator or its machine lattice. A general solution of Hill's equation can be written as

$$
y(s)=\sqrt{\epsilon \beta(s)} \sin (\psi(s)+\xi)
$$

with constant $\epsilon$ and $\xi$ determined by initial conditions.

\subsubsection{Transfer matrix}

An alternative approach to study the transverse motion is to trace the phase-space coordinate $\left(y, y^{\prime}\right)$ as the particle travels along the accelerator elements. The phasespace coordinate at both ends of an element is related by a symplectic map. For linear systems this map reduces to a $2 \times 2$ transfer matrix $M$ such that

$$
\left(\begin{array}{c}
y \\
y^{\prime}
\end{array}\right)_{s}=M\left(s, s_{0}\right)\left(\begin{array}{c}
y \\
y^{\prime}
\end{array}\right)_{s_{0}} .
$$

Transfer matrices of some common linear elements are listed below as examples

1. a drift space of length $l$

$$
M_{\mathrm{drift}}=\left(\begin{array}{ll}
1 & l \\
0 & 1
\end{array}\right)
$$

2. a sector dipole with bending radius $\rho$, bending angle $\theta$

$$
M_{\text {dipole }}=\left(\begin{array}{cc}
\cos \theta & \rho \sin \theta \\
-\frac{1}{\rho} \sin \theta & \cos \theta
\end{array}\right)
$$

3. a quadrupole of length $l$, focusing constant $K$

$$
\begin{aligned}
& M_{\text {quad }}(K>0)=\left(\begin{array}{cc}
\cos (\sqrt{K} l) & \frac{1}{\sqrt{K}} \sin (\sqrt{K} l) \\
-\sqrt{K} \sin (\sqrt{K} l) & \cos (\sqrt{K} l)
\end{array}\right) \\
& M_{\text {quad }}(K<0)=\left(\begin{array}{cc}
\cosh (\sqrt{K} l) & \frac{1}{\sqrt{K}} \sinh (\sqrt{K} l) \\
\sqrt{K} \sinh (\sqrt{K} l) & \cosh (\sqrt{K} l)
\end{array}\right)
\end{aligned}
$$


The transfer matrix of a section is the product of the transfer matrices of sequential elements, i.e.

$$
M(s+L, s)=M_{n} M_{n-1} \ldots M_{1} .
$$

The transfer matrix for a section described by Hill's equation (2.17) can be written explicitly

$$
M\left(s_{2}, s_{1}\right)=\left(\begin{array}{cc}
\sqrt{\beta_{2}} & 0 \\
-\frac{\alpha_{2}}{\sqrt{\beta_{2}}} & \frac{1}{\sqrt{\beta_{2}}}
\end{array}\right)\left(\begin{array}{cc}
\cos \psi & \sin \psi \\
-\sin \psi & \cos \psi
\end{array}\right)\left(\begin{array}{cc}
\frac{1}{\sqrt{\beta_{1}}} & 0 \\
\frac{\alpha_{1}}{\sqrt{\beta_{1}}} & \sqrt{\beta_{1}}
\end{array}\right),
$$

where subscript 1,2 indicate parameters at $s_{1}$ and $s_{2}$, respectively and $\psi=\psi\left(s_{2}\right)-$ $\psi\left(s_{1}\right)$ is the phase advance from $s_{1}$ to $s_{2}$.

The transfer matrix for a complete revolution can always be written in the form

$$
M=\left(\begin{array}{cc}
\cos \Phi+\alpha \sin \Phi & \beta \sin \Phi \\
-\gamma \sin \Phi & \cos \Phi-\alpha \sin \Phi
\end{array}\right)
$$

where the subscripts are dropped and $\Phi$ is phase advance in one revolution. The number of betatron oscillations in one revolution is defined as the betatron tune denoted by $Q_{y}$ or $\nu_{y}$

$$
Q_{y}=\nu_{y}=\frac{\Phi}{2 \pi}=\frac{1}{2 \pi} \int_{s}^{s+C} \frac{\mathrm{ds}}{\beta_{y}(s)} .
$$

\subsubsection{Emittances}

From Eq. (2.30) we see if we define

$$
\left(\begin{array}{c}
u \\
u^{\prime}
\end{array}\right)=\left(\begin{array}{cc}
\frac{1}{\sqrt{\beta}} & 0 \\
\frac{\alpha}{\sqrt{\beta}} & \sqrt{\beta}
\end{array}\right)\left(\begin{array}{c}
y \\
y^{\prime}
\end{array}\right)
$$

then

$$
\left(\begin{array}{l}
u_{2} \\
u_{2}^{\prime}
\end{array}\right)=\left(\begin{array}{cc}
\cos \psi & \sin \psi \\
-\sin \psi & \cos \psi
\end{array}\right)\left(\begin{array}{l}
u_{1} \\
u_{1}^{\prime}
\end{array}\right)
$$


In other words, the motion of $\left(u, u^{\prime}\right)$ in phase space is purely rotation about the origin, which means

$$
\begin{aligned}
u^{2}+u^{\prime 2} & =\frac{1}{\beta}\left(y^{2}+\left(\alpha y+\beta y^{\prime}\right)^{2}\right) \\
& =C\left(y, y^{\prime}\right)=\epsilon=\text { constant. }
\end{aligned}
$$

This constant is called the Courant-Snyder invariant. Eq. (2.33) can also be verified directly from Eq. (2.23). It specifies an ellipse in the $\left(y, y^{\prime}\right)$ plane which is the intersection of the Hamiltonian torus ${ }^{2}$ and the plane $\left(y, y^{\prime}\right)$ at each location $s$, as illustrated in Fig. 2.4. Since $\alpha, \beta$ are $s$-dependent, the shape and orientation of the ellipses are also $s$-dependent. The phase space area enclosed by the ellipse is $\pi \epsilon$. Particles will follow such ellipses according to their initial values of $\left(y_{0}, y_{0}^{\prime}\right)$ as they pass location $s$ consecutively.

Particles in a beam are distributed on the phase space plane and occupy a certain area. This phase space area is an important measure of the quality of the beam. The emittance measures the phase space area occupied by the beam. The rms beam emittance is defined as

$$
\epsilon_{\mathrm{rms}}=\sqrt{\sigma_{y}^{2} \sigma_{y^{\prime}}^{2}-\sigma_{y y^{\prime}}^{2}}
$$

where $\sigma_{y}, \sigma_{y^{\prime}}$ are rms beam widths and $\sigma_{y y^{\prime}}$ is the correlation of $y$ and $y^{\prime}$. The rms emittance can be thought as the phase space area enclose by the ellipse of an rms

\footnotetext{
${ }^{2}$ The Hill's equation can be derived from a pseudo-Hamiltonian

$$
H=\frac{1}{2} y^{2}+\frac{1}{2} K(s) y^{\prime 2}
$$
}

which gives rise to an invariant torus

$$
J=\frac{1}{2 \beta(s)}\left[y^{2}+\left(\beta(s) y^{\prime}+\alpha(s) y\right)^{2}\right]
$$

in the $\left(y, y^{\prime}\right)$ phase space. 
particle. The $95 \%$ emittance denotes the phase space area ${ }^{3}$ occupied by the core of the beam with $95 \%$ of the particles. For gaussian distribution, the $95 \%$ emittance and rms emittance are related by

$$
\epsilon_{95 \%}=6 \epsilon_{\mathrm{rms}}
$$

The emittance is related to the rms width of the beam. For gaussian beams, the relation is

$$
\epsilon_{\mathrm{rms}}=\frac{\sigma_{y}^{2}}{\beta_{y}}
$$

where $\beta_{y}$ is the beta function of the location at which beam width is measured. This relation does not take dispersion into account so it is valid only for the vertical plane.

In deriving the invariant ellipse equation we assumed constant momentum $p$ of the particles. If the beam is accelerated, a damping term, $p^{\prime} x^{\prime} / p$ has to be add to the lefthand side of Hill's equation (2.17), or equivalently we may use canonical momentum $p_{y}$ instead of $y^{\prime}=p_{y} / p$. So the true invariant is the normalized emittance

$$
\epsilon_{\mathrm{n}}=\gamma \beta \epsilon
$$

where $\gamma, \beta$ are Lorentz relativistic parameters.

\subsubsection{Dispersion}

The discussion about the transverse motion so far is for on-momentum particles. However most particles in a beam are off-momentum, i.e., having momentum $p \neq p_{0}$. Since the bending radii in a dipole are larger for particles with momentum $p>p_{0}$ and smaller for those with $p<p_{0}$, the off-momentum orbits have deviations from the

\footnotetext{
${ }^{3}$ Usually the area is divided by $\pi$ and the unit of $\pi$-mm-mrad is used to measure phase space area.
} 
on-momentum orbit. Similarly, the focusing effects of quadrupoles also depend on the particle momentum and this affects the betatron motion of the off-momentum particles.

The momentum deviation $\delta$ is defined as

$$
\delta=\frac{\Delta p}{p}, \quad \Delta p=p-p_{0}
$$

Then the bending radius for an off-momentum particle is $\rho /(1+\delta)$ with $\rho$ the onmomentum radius. Expanding Eq. (2.13) in terms of $x, \delta$ and keeping only linear terms, the equation of motion becomes [1]

$$
\begin{aligned}
x^{\prime \prime}+\left(K_{x}+\Delta K_{x}\right) x= & \frac{\delta}{\rho}, \\
K_{x}=\frac{1}{\rho^{2}}-K_{1}(s), & \Delta K_{x}=\left(-\frac{2}{\rho^{2}}+K_{1}(s)\right) \delta .
\end{aligned}
$$

The inhomogeneous term on the right-hand side gives rise to orbit deviations and the $\Delta K_{x}$ term represents the momentum-dependence of the focusing effect which causes chromatic aberration to be discussed in the next subsection.

A solution of Eq. (2.40) can be written in the form

$$
x=x_{\beta}(s)+D(s) \delta
$$

with

$$
\begin{aligned}
x_{\beta}^{\prime \prime}+K_{x}(s) x_{\beta} & =0, \\
D^{\prime \prime}+K_{x}(s) D & =\frac{1}{\rho},
\end{aligned}
$$

where the chromatic term $\Delta K_{x}$ is omitted here. So the off-momentum particle is undergoing betatron oscillation around a deviated closed orbit. The $x_{\beta}$ term corresponds to betatron motion and $D(s) \delta$ is the deviation from the on-momentum closed orbit. The periodic condition $D(s+C)=D(s)$ is imposed to ensure the offset of the 
reference orbit is also closed. Function $D(s)$ is called the dispersion function. The dispersion function is usually vanishing for the vertical plane because the bending is only in the horizontal plane.

In presence of non-zero dispersion, the beam width depends on its momentum spread as well as beam transverse emittance. Assuming that there is no correlation between the longitudinal and horizontal distribution, the relation is

$$
\sigma_{x}^{2}=\epsilon_{x} \beta_{x}+D^{2} \sigma_{\delta}^{2}
$$

where $\epsilon_{x}$ is horizontal $\mathrm{rms}$ emittance and $\sigma_{\delta}$ is rms momentum spread.

Because an off-momentum particle takes a different path than the on-momentum particle, the total path length usually depends on the momentum deviation by a term

$$
\Delta C=\left[\oint \frac{D(s)}{\rho} \mathrm{ds}\right] \delta .
$$

The momentum compaction factor $\alpha_{c}$ is defined as

$$
\alpha_{c}=\frac{1}{C} \frac{\mathrm{d} \Delta \mathrm{C}}{\mathrm{d} \delta} .
$$

The revolution period $T=C / v$ also depends on momentum by

$$
\frac{\Delta T}{T}=\frac{\Delta C}{C}-\frac{\Delta v}{v}=\left(\alpha_{c}-\frac{1}{\gamma^{2}}\right) \frac{\Delta p}{p}=\eta \delta,
$$

where the phase-slip factor $\eta$ is defined as

$$
\eta=\alpha_{c}-\frac{1}{\gamma^{2}}
$$

For a particular energy corresponding to

$$
\gamma_{\mathrm{T}}=\sqrt{1 / \alpha_{c}}
$$

the revolution frequency is independent of momentum deviation. This energy is called the transition energy. The phase slip factor and transition energy are of great importance for the longitudinal motion of the beam. 


\subsubsection{Chromaticity}

The focusing strength deviations $\Delta K_{x, z}$ term are proportional to momentum deviation,

$$
\Delta K_{x} \approx-K_{x} \delta, \quad \Delta K_{z} \approx-K_{z} \delta
$$

which correspond to "gradient errors" for off-momentum particles. The error of the focusing strength causes tune shifts. The ratio of tune shift to momentum deviation is called chromaticity, in particular, natural chromaticity, if the tune shift is due to error of quadrupole focusing strengths only. The natural chromaticity is defined as

$$
C_{y, \text { nat }} \equiv \frac{\Delta \nu_{y}}{\delta}=-\frac{1}{4 \pi} \oint \beta_{y} K_{y} \mathrm{ds}
$$

The natural chromaticity is usually in the order of $-\nu_{y}$. The large tune spread due to chromaticity is sometime dangerous because it can sit a fraction of the beam on a resonance line. It is often necessary to correct the chromaticity. This can be achieved using sextupoles since the orbit offsets of the off-momentum particles in sextupole magnetic field contribute a focusing force that is proportional to $D \delta$. Including the sextupole corrections, the chromaticity is

$$
\begin{aligned}
C_{x} & =-\frac{1}{4 \pi} \oint \beta_{x}\left(K_{x}+K_{2} D\right) \mathrm{ds}, \\
C_{z} & =-\frac{1}{4 \pi} \oint \beta_{z}\left(K_{z}-K_{2} D\right) \mathrm{ds},
\end{aligned}
$$

where $K_{2}(s)$ is the sextupole component.

\subsection{Longitudinal Motion}

RF cavities provide energy to the beam through the longitudinal electric field across their gaps. The electric field oscillates sinusoidally with radio frequency. Only particles that arrive at the rf gap at the right time (i.e. with right phases) can gain energy. 
The longitudinal motion of the particles must be synchronized with the rf phase to ensure that particles always gain energy. Consequently the oscillation frequency of the rf cavities must be an integral multiple of the revolution frequency of the beam.

We assume a particle which always arrives at the rf gap with the same phase. We call this particular particle the synchronous particle and define the phase as the synchronous phase. The revolution frequency of a particle depends on its momentum. A particle with momentum different than the synchronous particle will become out of phase with the rf eventually. However, if the rf phase is set properly, particles with higher momentum will gain less energy from the cavities and thus be pulled back to the synchronous particle and vice versa. In this case, a typical particle oscillates around the synchronous particle. This is the longitudinal motion to be discussed in this section.

\subsubsection{Equations of motion}

The electric field across the gap of an rf cavity is of the form

$$
\mathcal{E}=\mathcal{E}_{0} \sin \left(h \omega_{0} t+\phi_{s}\right)
$$

where $\omega_{0}$ and $\phi_{s}$ are the angular revolution frequency and the phase angle of the synchronous particle, respectively and $h$ is the harmonic number. The synchronous particle crosses the gap at time $t=0$ and sees a voltage $V \sin \phi_{s}$.

Suppose the synchronous particle has energy $E$, a general particle has energy $E+\Delta E$ and sees an rf phase $\phi$. The mapping relation of the longitudinal coordinate $(\phi, \Delta E)$ of a general particle for two consecutive passes is [1]

$$
\begin{aligned}
\Delta E_{n+1} & =\Delta E_{n}+e V\left(\sin \phi_{n}-\sin \phi_{s}\right), \\
\phi_{n+1} & =\phi_{n}+\frac{2 \pi h \eta}{\beta^{2} E} \Delta E_{n+1},
\end{aligned}
$$


where subscript $n, n+1$ denote the $n$ th, $n$th +1 pass. When the acceleration rate is low, the mapping equation can be approximated by differential equations

$$
\begin{aligned}
\frac{\mathrm{d}}{\mathrm{dt}}\left(\frac{\Delta E}{\omega_{0}}\right) & =\frac{1}{2 \pi} e V\left(\sin \phi-\sin \phi_{s}\right), \\
\frac{\mathrm{d} \phi}{\mathrm{dt}} & =\frac{h \omega_{0}^{2} \eta}{\beta^{2} E}\left(\frac{\Delta E}{\omega_{0}}\right)
\end{aligned}
$$

with coordinate $\left(\phi, \Delta E / \omega_{0}\right)$, or

$$
\begin{aligned}
\dot{\delta} & =\frac{\omega_{0}}{2 \pi \beta^{2} E} e V\left(\sin \phi-\sin \phi_{s}\right) \\
\dot{\phi} & =h \omega_{0} \eta \delta
\end{aligned}
$$

with coordinate $(\phi, \delta)$, where the dot indicates the derivative with respect to time $t$. The above equations can be derived from a Hamiltonian

$$
H=\frac{1}{2} h \omega_{0} \eta \delta^{2}+\frac{\omega_{0} e V}{2 \pi \beta^{2} E}\left[\cos \phi-\cos \phi_{s}+\left(\phi-\phi_{s}\right) \sin \phi_{s}\right] .
$$

\subsubsection{RF phase stability and rf bucket}

The equations of motion Eqs. (2.59), (2.60) lead to

$$
\ddot{\phi}-\frac{h \omega_{0}^{2} e V \eta}{2 \pi \beta^{2} E}\left(\sin \phi-\sin \phi_{s}\right)=0
$$

which is reduced to

$$
\ddot{\phi}-\omega_{0}^{2} \frac{h e V \eta \cos \phi_{s}}{2 \pi \beta^{2} E}\left(\phi-\phi_{s}\right)=0
$$

when the phase error $\phi-\phi_{s}$ is small. It is seen that the longitudinal motion is stable only if

$$
\eta \cos \phi_{s}<0
$$

In other words, the phase stability condition is $0 \leq \phi_{s}<\pi / 2$ for $\eta<0$ (before transition) and $\pi / 2<\phi_{s} \leq \pi$ for $\eta>0$ (after transition). The phase stability 
condition can be understood as follows. Below the transition energy, a particle with higher energy completes the revolution with less time and thus arrives earlier than the synchronous particle. When $\phi_{s}<\pi / 2$, particles see the rising slope of rf wave and therefore pick up less energy than the synchronous particle. So its phase is pulled back to the synchronous particle. Similarly a particle with lower energy picks up more energy than the synchronous particle and thus its phase catches up with the synchronous particle. Therefore the beam is stable under acceleration. Above the transition energy, a higher energy particle takes more time to complete one revolution and lags behind the synchronous particle. The beam stays stable only if the particles see the falling slope, i.e., $\pi / 2<\phi_{s} \leq \pi$. The condition is illustrated in Fig. 2.5.

The Hamiltonian Eq. (2.61) specifies the longitudinal dynamics in the $(\phi, \delta)$ phasespace. Since the synchrotron Hamiltonian is time-independent, it is a constant of motion. Particles follow curves of constant Hamiltonian $H$ (i.e., Hamiltonian tori) according to their initial conditions. The Hamiltonian torus that passes the unstable fixed point (UFP) at $\left(\pi-\phi_{s}, 0\right)$ is called the separatrix. The separatrix divides the synchrotron phase space into stable and un-stable regions. The tori inside the separatrix are closed which means the motion is stable. The tori outside the separatrix are open. Particles on open tori will not be kept synchronized with the rf and will be lost during acceleration eventually. The phase space area enclosed by the separatrix is called the rf bucket area. Particles inside the rf bucket oscillate around the stable fixed point $(\mathrm{SFP})$ at $\left(\phi_{s}, 0\right)$ and remain in phase with the rf on average. These particles are grouped together by the rf and form a bunch. Since only these particles survive the acceleration, the beams in synchrotrons are bunched. Fig. 2.6 shows examples of the separatrix and stable/un-stable regions in $(\phi, \delta)$ phase space with different settings. The current of a bunched beam observed with a pickup reflects the bunch structure. The bunching factor $B_{f}$ is defined as the ratio of peak current to average current. For 

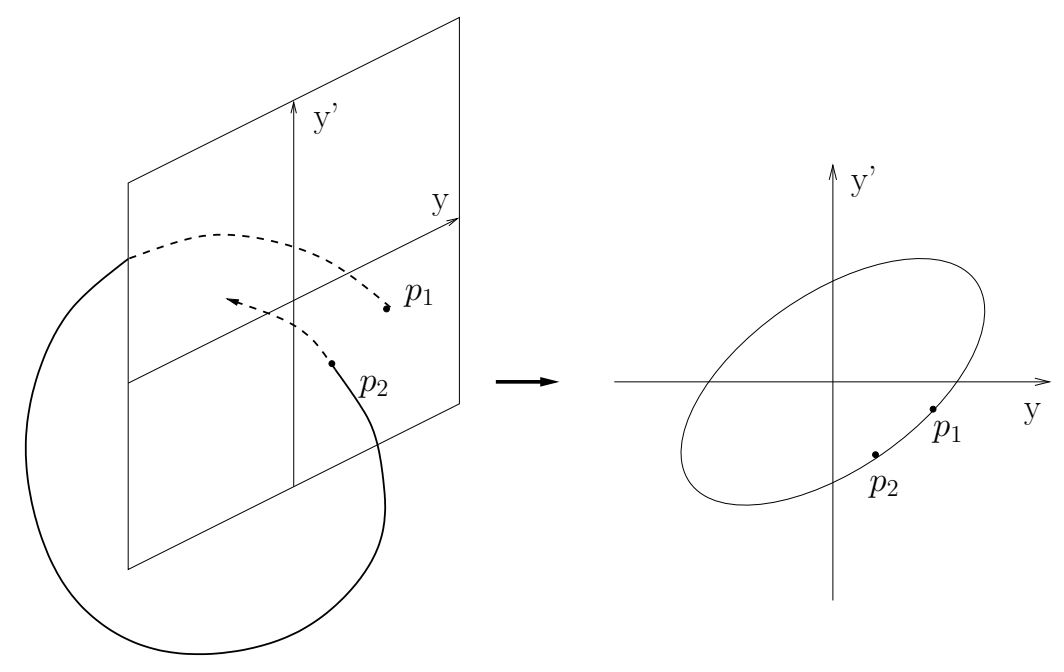

Figure 2.4: The Poicare section.

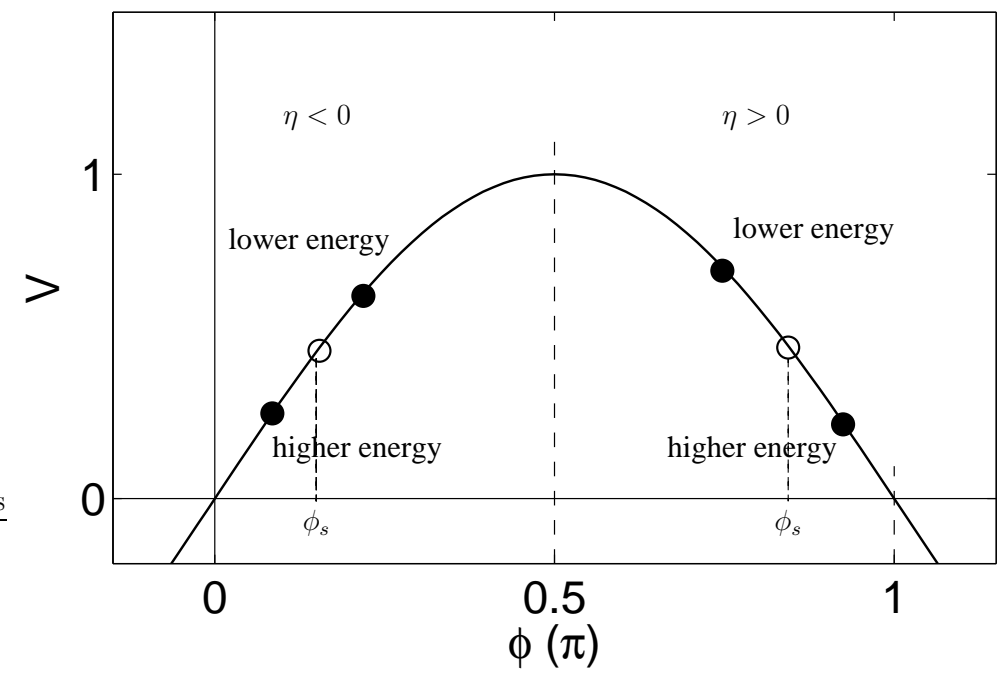

Figure 2.5: The rf phase stability condition. 
gaussian beams,

$$
B_{f}=\frac{\sqrt{2 \pi}}{\sigma_{\phi}},
$$

where $\sigma_{\phi}$ is the rms bunch length.

The motion of particles with small oscillation amplitude are simple harmonic oscillation with angular synchrotron frequency $\omega_{s}$ which are given below with the definition of the synchrotron tune $Q_{s}$

$$
\omega_{s}=\omega_{0} \sqrt{\frac{h e V \eta \cos \phi_{s}}{2 \pi \beta^{2} E}}, \quad Q_{s}=\frac{\omega_{s}}{\omega_{0}} .
$$

The corresponding Hamiltonian tori are ellipses centered at the SFP. The maximum momentum deviation $\hat{\delta}$ and maximum phase error $\hat{\phi}$ of the ellipses are related by

$$
\frac{\hat{\delta}}{\hat{\phi}}=\frac{Q_{s}}{h|\eta|} .
$$

However, particles with large oscillation amplitude follow Eq. (2.62). The oscillation frequency decreases with increased amplitude.

\subsubsection{Longitudinal emittance}

Typically particles are populated in a fraction of the rf buckets around the SFP. For an equilibrium gaussian distribution, the phase space area the beam occupies is related to the rms momentum spread $\sigma_{\delta}$ and rms bunch length $\sigma_{\phi}$ by

$$
\tilde{A}_{\mathrm{rms}}=\pi \sigma_{\delta} \sigma_{\phi}, \quad \tilde{A}_{0.95}=6 \tilde{A}_{\mathrm{rms}},
$$

where $\tilde{A}_{\text {rms }}$ is the rms phase space area and $\tilde{A}_{0.95}$ is the $95 \%$ phase space area. The synchrotron phase-space area is often measured in $\left(\phi / h, \Delta E / \omega_{0}\right)$ phase space. Its value $A$, in unit of $\mathrm{eV} \cdot \mathrm{s}$, is related to the value $\tilde{A}$ by

$$
\tilde{A}=A \frac{h \omega_{0}}{\beta^{2} E}
$$




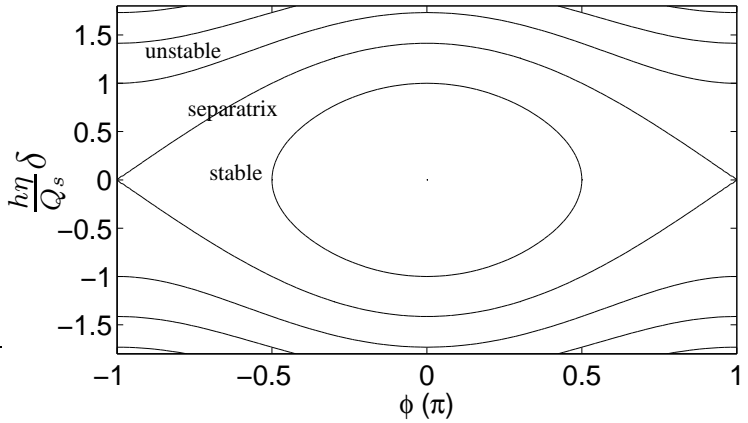

(a) $\phi_{s}=0, \eta<0$

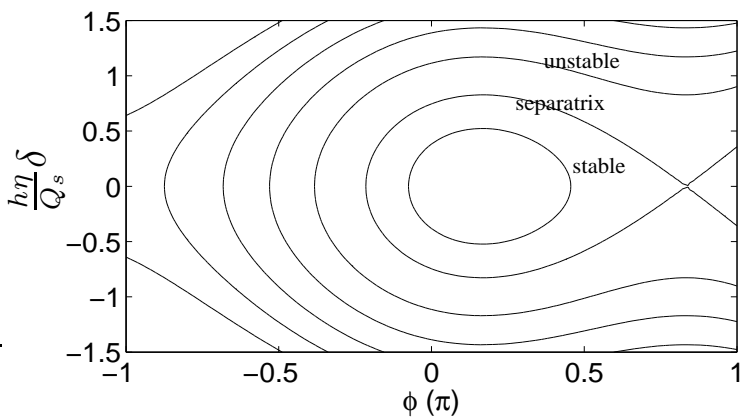

(b) $\phi_{s}=\pi / 6, \eta<0$

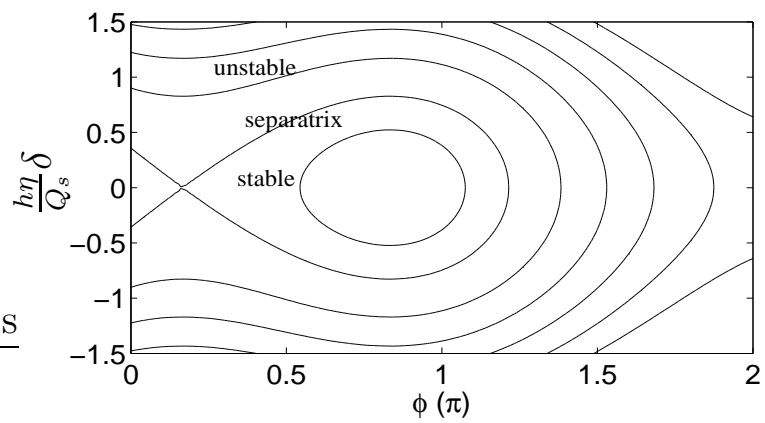

(c) $\phi_{s}=5 \pi / 6, \eta>0$

Figure 2.6: Synchrotron Hamiltonian tori in different settings. (a) Stationary bucket below transition. (b) Moving bucket below transition. (c) Moving bucket above transition. 
The synchrotron phase-space area is an important indication of the beam quality. It is also referred as longitudinal emittance.

If the phase-space distribution of a bunch, or the bunch shape, matches the Hamiltonian ellipses, it will stay matched as the oscillations of all particles follow the ellipses. The projection onto either the $\delta$ or $\phi$ direction will remain unchanged. However, if it is mismatched, the bunch shape will be constantly changing as each particle follow its own ellipse. In this case, the bunch length and the peak current will oscillate with twice of the synchrotron frequency. The matched and mismatched beam are illustrated in Fig. 2.7.

\subsubsection{Transition crossing}

The longitudinal phase stability condition Eq. (2.64) requires a sudden change of synchronous phase from $\phi_{s}$ to $\pi-\phi_{s}$ when the phase slip factor $\eta$ crosses zero, as indicated in Fig. 2.5. This happens for synchrotrons whose transition energy is within the beam energy range. As the beam energy crosses the transition energy, it causes some important complication to the operation of a synchrotron. This is called transition crossing.

Recalling that $\frac{\Delta T}{T}=\eta \frac{\Delta p}{p}$ and $\eta=1 / \gamma_{T}^{2}-1 / \gamma^{2}$, we see transition crossing corresponds to $\gamma=\gamma_{T}$ and at transition crossing the revolution time is momentum independent. The longitudinal motion is normally adiabatic because the longitudinal Hamiltonian varies very slowly with time so that it can be considered as timeindependent. This adiabatic condition is not satisfied near transition region because when the phase slip factor $\eta$ is very small, its linear growth term due to acceleration becomes significant. The linear non-adiabatic motion near transition causes the Hamiltonian tori to tilt in phase space. The momentum dependence term of $\eta$ also becomes important near transition. Expanding to linear term with respect to 

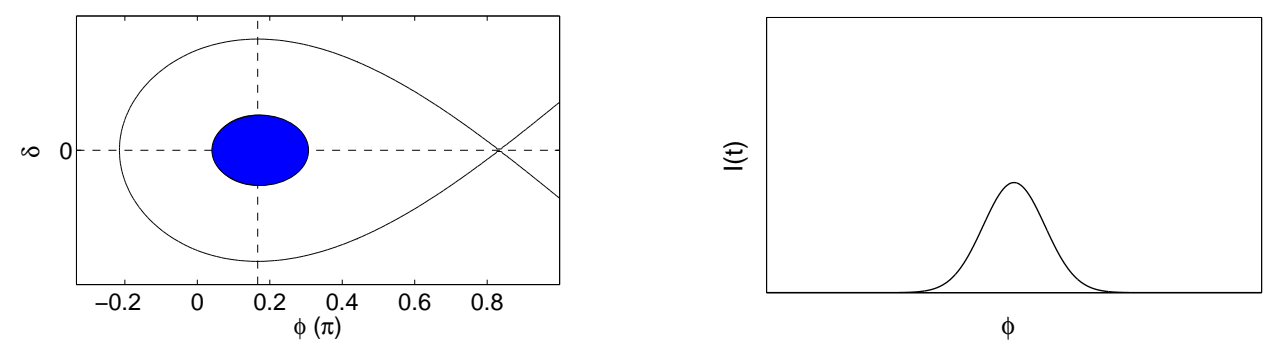

(a) matched beam
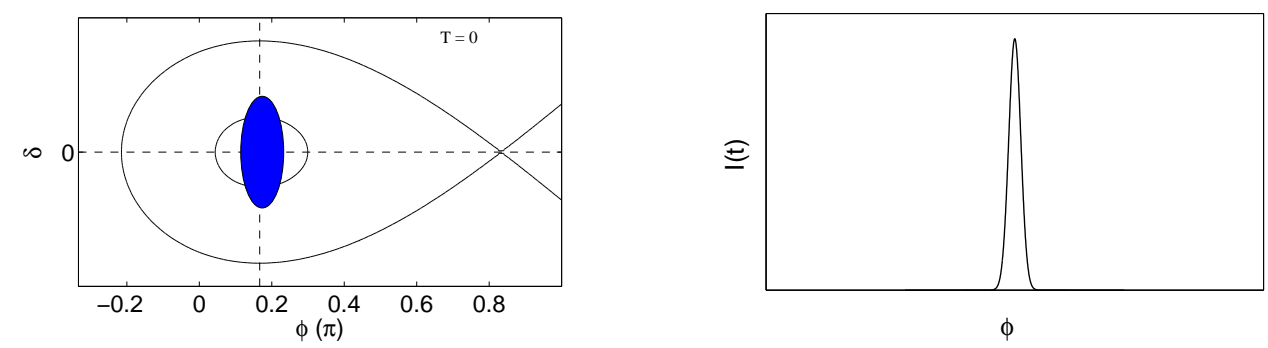

(b) mismatched beam, $T=0$
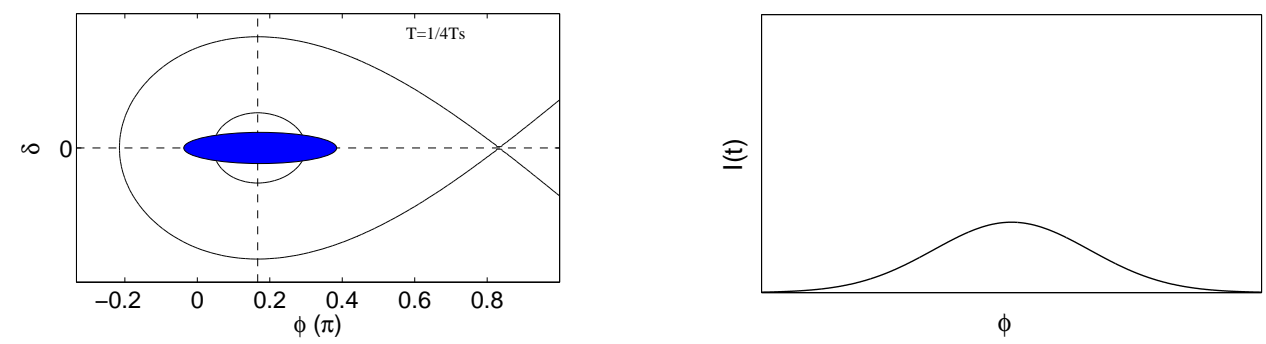

(c) mismatched beam, $T=T_{s} / 4$

Figure 2.7: Left: Schematics of matched and mismatched beam in rf buckets. Right: charge density distribution on the $\phi$ direction. (a) matched bunch. (b) mismatched bunch; (c) the mismatched bunch after $1 / 4$ synchrotron period. 
momentum $\delta$, the phase slip factor is

$$
\eta=\frac{2 \dot{\gamma} t}{\gamma_{T}^{3}}+\eta_{1} \delta
$$

The $\eta_{1} \delta$ term means that particles with different momentum deviation crosses transition at slightly different time. Since the beam always has momentum spread, the synchrotron motion is unstable for a portion of the beam during transition. This causes the longitudinal emittance to grow.

At transition the rf bucket becomes very large in the momentum direction (i.e., large momentum acceptance), the phase space ellipse becomes very thin in the $\phi$ direction, corresponding to very short bunches. The short bunches have large space charge effect for intense beams, which could cause microwave instability and beam loss. The longitudinal space charge effect also perturbs the rf potential well and distorts the phase space ellipses. Below transition, space charge effect is a longitudinal defocusing force. Above transition, it is a focusing force since $\eta$ changes sign. So the phase space distortion due to space charge effect is different across the transition energy. It causes phase space mismatch and a quadrupole mode oscillation after transition as shown in Fig. 2.7. Such oscillations with large amplitude can also lead to beam loss. 


\section{Chapter 3}

\section{Application of Orbit Response Measurements at Fermilab Booster}

\subsection{Introduction}

Linear optics is one of the most important properties of a synchrotron. Depending on the purpose of the synchrotron, it affects the luminosity or brightness, beam life time, beam loss, injection efficiency, operations of diagnostics, etc. The linear optics is determined by the placement and strengths of the dipole and quadrupole magnets which are often in a repetitive structure called the lattice of the machine. We usually rely on computer programs such as MAD [9], ELEGANT [10], COMFORT, etc. to analyze the lattice model and study the linear optics. The lattice model is based on the design model or direct measurements, including surveys and magnet field measurements. However, the model parameters may not thoroughly represent the real machine because of various errors that can be introduced during construction and operation. Hence it is very desirable to build a realistic model that represents the actual machine as seen by the beam. The model parameters have to be calibrated 
with beam-based measurements. The orbit response matrix (ORM) method [8] is one approach to achieve this goal.

The closed-orbit of a synchrotron is mainly determined by the dipole magnets around the ring. The beam centroid follows this orbit if it is on-momentum and there is no betatron excitation. Perturbations of the magnetic dipole field causes global orbit deviations according to the actual machine lattice. The orbit deviations due to changes of localized dipole magnets (i.e., trim dipoles) are called orbit responses or differential orbits. It can be easily measured with beam position monitors (BPMs). On the other hand, orbit responses can be predicted with the lattice model. The orbit response matrix (ORM) method measures the orbit responses around the ring using many trim dipoles. The model parameters are then calibrated by minimizing the differences between the measurements and model predictions.

The ORM method has been successfully applied to many synchrotrons, mostly electron storage rings [11]. It has been used to locate and correct or compensate the errors of the lattice. These studies enabled the detection of wiring mistakes and magnet imperfections and restored design lattice periodicity to improve the performance of the machines by reducing the beam emittance and increasing the beam lifetime. The ORM method also obtains information to calibrate the BPMs and the trim dipole magnets. The method has been more successful for electron storage rings because of their high orbit stability and the high precision of orbit measurements. The Fermilab Booster is a fast-ramping synchrotron whose beam energy ramps up from $400 \mathrm{MeV}$ to $8 \mathrm{GeV}$ in $33 \mathrm{msec}$. Since the steering magnets are not ramped up and even the main dipole magnets can be mismatched with the beam momentum, the beam orbit drifts around swiftly during the cycle, especially in the horizontal plane. The orbit also varies from cycle to cycle due to different initial injection conditions. The BPM resolution is not very high. These factors contribute to the difficulties of applying ORM to the Booster. 
A more specific difficulty arises from the fact that the Booster is composed of combined-function magnets. Since the quadrupole components are distributed in a large portion of the entire ring and are very close to the adjacent magnets, they perturb the lattice structure in very similar patterns. The ORM doesn't have enough information to distinguish the neighboring magnets. Thus it is very hard or even impossible to separate the effects of these adjacent magnets. This places a significant limitation to the Booster ORM problem. It also introduces big uncertainties to the solutions that have to be solved by imposing constraints to the solutions. However, our study shows that although the individual parameters of the combined-function magnets cannot be fully determined, the solution still provides useful insights into the real Booster because some simple combinations (sum or difference) of these parameters are well constrained and can be used to infer the status of the real Booster.

Because of the reduced accuracy of orbit measurements and the specific nature of the Booster ORM problem described above, the least-square solving algorithm of Ref [8] often fails to produce a reasonable solution to proceed. We had to adopt a more robust algorithm to overcome this difficulty.

Our constrained fitting scheme reduces the normalized $\chi^{2}$ from the initial value of near 80 down to near 2.5, while the expected final $\chi^{2}$ is 1.0 if the final difference is entirely from random noise. The solution suggests that gradient errors of the magnets come from the sextupole focusing effects due to beam orbit offsets in the sextupole components of the combined-function magnets. 


\subsection{The ORM Fitting Model and Algorithm}

\subsubsection{The unconstrained fitting scheme}

The ORM measures the closed orbit deviations due to dipole kicks. Let $\Delta y_{i}$ be the orbit deviation at the $i$ 'th BPM due to a kick angle $\Delta \theta_{j}$ of the $j$ 'th dipole kicker ${ }^{1}$, then the corresponding ORM element is

$$
M_{i j}=\frac{\Delta y_{i}}{\Delta \theta_{j}},
$$

where $y$ denotes horizontal orbit $x$ or vertical orbit $z$ and $\Delta \theta_{j}$ denotes a horizontal kick or a vertical kick. The matrix dimension is $m \times K$ if there are $m$ BPMs and $K$ kickers. A horizontal kicker causes orbit changes mainly in the same transverse plane and so does a vertical kicker. However a kicker can perturb the orbit in the other transverse plane if the dipole or quadrupole magnets are rolled about their axis. The rolls of the BPMs and kickers also add up to the observations of cross-plane orbit deviations. The uncoupled ORM refers to the matrix which contains the in-plane orbit responses only and the coupled ORM contains both in-plane and cross-plane orbit deviations.

The Booster has 48 BPMs which are all able to measure orbits in both transverse planes. And it has 48 corrector packages, each of which contains a horizontal trim dipole and a vertical trim dipole. So the coupled ORM is $96 \times 96$ in dimension, which may be divided into 4 blocks

$$
\mathbf{M}=\left(\begin{array}{ll}
M_{x x} & M_{x z} \\
M_{z x} & M_{z z}
\end{array}\right)
$$

\footnotetext{
${ }^{1}$ In the ORM literature, a "kicker" refers to a short dipole magnet which introduces small, localized orbit deflections called "kicks". For the Booster, we often call it a trim dipole or simply a trim.
} 
with the two diagonal blocks representing orbit deviations due to in-plane kicks and the off-diagonal blocks representing orbit deviations due to cross-plane kicks. The BPM and kickers in each straight section are practically in the same location and can be labeled with the same index which runs from 1 to 48 .

The uncoupled ORM is directly related to beta functions and phase advances at the locations of BPMs and kickers by [1]

$$
M_{i j}=\frac{\sqrt{\beta_{i} \beta_{j}}}{2 \sin \pi \nu} \cos \left(\pi \nu-\left|\psi_{i}-\psi_{j}\right|\right),
$$

which is just the Green's function of Hill's equation (2.17). The fully-coupled model ORM can be derived from the 4D transfer matrices using the closed-orbit condition. Let $\mathbf{T}$ be the $4 \mathrm{D}$ one-turn transfer matrix at the location of a horizontal kicker with kick $\theta$, the closed-orbit condition is

$$
\mathbf{T}\left(\begin{array}{c}
\Delta x_{0} \\
\Delta x_{0}^{\prime} \\
\Delta z_{0} \\
\Delta z_{0}^{\prime}
\end{array}\right)=\left(\begin{array}{c}
\Delta x_{0} \\
\Delta x_{0}^{\prime}-\theta \\
\Delta z_{0} \\
\Delta z_{0}^{\prime}
\end{array}\right),
$$

which enables us to solve for change of phase space coordinate per unit kick angle $\left(\Delta x_{0} / \theta, \Delta x_{0}^{\prime} / \theta, \Delta z_{0} / \theta, \Delta z_{0}^{\prime} / \theta\right)$ at the kicker's location. It can be propagated to other locations using the 4D transfer matrices and the ORM elements are then determined according to Eq. (3.1). The same calculation can be done for a vertical kicker by changing $-\theta$ to the $\Delta z_{0}^{\prime}$ component in Eq. (3.3).

One other choice is to let a computer program, e.g., MAD, calculate the closedorbit before and after the kick is applied. This would include the focusing effects of sextupoles and the steering effects of quadrupoles when the beam is off-center. Since this method requires model evaluation once per kicker, it costs significantly longer calculation time than the transfer matrix method, especially in Jacobian matrix calculation. We therefore use the transfer matrix method in this study. 
The differences between the measured ORM and the calculated model ORM come not only from the errors in the existing lattice model, but also from the imperfections of the measurement system, including the calibration factors (gains) and rolls of the BPMs and trim dipoles, and the momentum deviation caused by horizontal kicks. The effects of the BPM parameters are taken into account by

$$
\left(\begin{array}{c}
\Delta x \\
\Delta z
\end{array}\right)_{i}^{\text {act }}=B_{i}\left(\begin{array}{c}
\Delta x \\
\Delta z
\end{array}\right)_{i}^{\text {meas }}, \quad B_{i} \equiv\left(\begin{array}{cc}
\cos \theta & \sin \theta \\
-\sin \theta & \cos \theta
\end{array}\right)\left(\begin{array}{cc}
1 / b_{h} & 0 \\
0 & 1 / b_{v}
\end{array}\right)
$$

where $b_{h}, b_{v}$ are horizontal and vertical gains and $\theta$ is the roll of the BPM. Superscripts "act" and "meas" represent the corrected values and raw measurements, respectively. The kicker parameters are

$$
\left(\begin{array}{c}
\Delta \theta_{x} \\
\Delta \theta_{z}
\end{array}\right)_{i}^{\text {act }}=K_{j}\left(\begin{array}{c}
\Delta \theta_{x} \\
\Delta \theta_{z}
\end{array}\right)_{i}^{\text {meas }}, \quad K_{j} \equiv\left(\begin{array}{ll}
k_{h} \cos \phi_{h} & k_{v} \sin \phi_{v} \\
k_{h} \sin \phi_{h} & k_{v} \cos \phi_{v}
\end{array}\right)
$$

where $k_{h}$ and $\phi_{h}$ are gains and rolls of horizontal trims, $k_{v}$ and $\phi_{v}$ are gains and rolls of vertical trims. The additional orbit changes due to momentum deviations caused by the horizontal kickers should be subtracted from the measured orbit. Let $\delta_{j}$ denote the induced momentum deviation of the $j$ 'th horizontal kicker and $D_{i}$ denote the dispersion function at the $i$ 'th BPM, then the corrected ORM is found from

$$
\left(\begin{array}{cc}
M_{x x} & M_{x z} \\
M_{z x} & M_{z z}
\end{array}\right)_{i j}^{\mathrm{act}}=B_{i}\left[\left(\begin{array}{cc}
M_{x x} & M_{x z} \\
M_{z x} & M_{z z}
\end{array}\right)_{i j}^{\text {meas }}-D_{i} \delta_{j}\left(\begin{array}{cc}
k_{h} \cos \phi_{h} & k_{v} \sin \phi_{v} \\
0 & 0
\end{array}\right)\right] K_{j}^{-1}
$$

where index $i$ and $j$ run from 1 to 48 .

The BPM and kicker parameters, or correction parameters, are unknowns in the ORM fitting model. The parameters in the lattice model are the gradient errors and the rolls of all 96 combined-function magnets. There are a total of 576 fitting parameters. 
The difference between the model and measured (with correction) ORM is characterized by the residual vector $\mathbf{r}$ which is a column vector defined by

$$
r_{k}=\frac{M_{i j}^{\text {act }}-M_{i j}^{\text {model }}}{\sigma_{i j}}, \quad k=(j-1) K+i,
$$

where $K=96$ is the number of kickers, $i, j$ run over all BPM and kicker indices and $\sigma_{i j}$ denotes the uncertainty of the corresponding measured element, and the objective function

$$
\chi^{2}=\mathbf{r}^{T} \mathbf{r}
$$

The residual vector can be extended with the betatron tunes by including the terms $\left(\nu_{x}^{\text {meas }}-\nu_{x}^{\text {model }}\right) / \sigma_{\nu, x}$ and $\left(\nu_{z}^{\text {meas }}-\nu_{z}^{\text {model }}\right) / \sigma_{\nu, z}$. We may also include the dispersion function measurements if available. The dispersion terms are $\left(D_{i}^{\text {meas }}-D_{i}^{\text {model }}\right) /\left(b_{h, i} \sigma_{D, i}\right)$. Putting all fitting parameters in a column vector $\alpha$, The ORM problem is in the mathematical form of minimizing the function $f(\alpha)=\chi^{2}$, which is a nonlinear least-square problem.

An iterative approach with the trust-region searching strategy [12, 13] is taken to solve this problem. For each iteration the Jacobian matrix $\mathbf{J}$ defined by

$$
J_{i j}=\frac{\partial r_{i}}{\partial \alpha_{j}}
$$

is computed as a first step. The advance of $\alpha$ on the basis of the last iteration is found by solving the following equation

$$
\left(\mathbf{J}^{T} \mathbf{J}+\lambda \mathbf{I}\right) \Delta \alpha=-\mathbf{J}^{T} \mathbf{r},
$$

where $\mathbf{I}$ is the identity matrix and $\lambda$ is a parameter to be scaled up or down depending on whether the solution $\Delta \alpha$ brings $\chi^{2}$ down. This Levenberg-Marquardt method [12, $13]$ is more robust than the algorithm suggested in Ref. [8], which solves $\mathbf{J} \Delta \alpha=-\mathbf{r}$ with singular value decomposition (SVD) instead. The Levenberg-Marquardt method 
can take a steepest-descent path or a hybrid searching direction if the normal equation does not predict a reasonable solution, which happens when the linear approximation is not good enough. This method is also faster because the eigen-decomposition of matrix $\mathbf{J}^{T} \mathbf{J}+\lambda \mathbf{I}$ takes much less time to compute than the SVD of $\mathbf{J}$ due to the bigger size of the latter. The error bars of the fitting parameters are estimated by computing the covariance matrix

$$
\mathbf{C}=\mathbf{J}^{T} \mathbf{J}
$$

and then error bar is

$$
\sigma_{i}=\sqrt{C_{i i}}
$$

for the $i$ 'th parameter.

\subsubsection{Simulation with the PSR model}

The above fitting scheme would be an efficient method for model calibration with ORM data. We checked the applicability of the method by simulations with two synchrotron models, the LANL PSR and Fermilab Booster.

The simulations are conducted by generating "measured" ORM with certain simulation parameters and applying the fitting scheme to the ORM to obtain solutions. If the solutions recover the simulation parameters, then the fitting algorithm has achieved its goal successfully. The simulation parameters are set to random values with realistic distributions. Gaussian random noise can be added to the simulated ORM.

PSR is a proton storage ring with a circumference of $90.2 \mathrm{~m}$ and beam energy of $800 \mathrm{MeV}$. The PSR lattice is composed of 10 periods of FODO cells. The nominal horizontal betatron tune is 3.2 and the vertical betatron tune is 2.2 . There are 19 BPMs which measure both horizontal and vertical orbit. There are 9 vertical kickers. 
To study the roll parameters, we assume PSR has 9 horizontal kickers which are installed beside the vertical kickers just like the Fermilab Booster so the fully-coupled model can be directly used. The ORM is $38 \times 18$ by size and there are a total of $20 \times 2+19 \times 3+9 \times 4=133$ fitting parameters

The simulation parameters of the PSR model are set to random values as follows: (1) quadrupole gradients error $\Delta K_{1}$ within $[-0.02,0.02] \mathrm{m}^{-2} ;(2)$ quadrupole rolls $\theta$ within $[-10,10]$ mrad; (3) BPM gains and kicker gains within $[0.9,1.1]$; (4) BPM and kicker rolls within $[-10,10]$ mrad. Initially the parameters are set to their default values, i.e., all ones for gains and all zeros otherwise. The initial normalized $\chi^{2}$ is 627.6 (uncertainty level of matrix elements is set to $0.05 \mathrm{~m} / \mathrm{rad}$ in $\chi^{2}$ calculation.). It is reduced to $1.0 \times 10^{-7}$ in 5 iterations while the algorithm converged to the expected solution precisely.

We then add gaussian noises to the matrix elements with $\sigma=0.05 \mathrm{~m} / \mathrm{rad}$, which corresponds to BPM resolution of $0.1 \mathrm{~mm}$ and kick angle of $2 \mathrm{mrad}$. The initial normalized $\chi^{2}$ is 628.6. It is reduced to 0.9 in 5 iterations when converged. The history of $\chi^{2}$ for both cases, with or without noises, is plotted in Fig. 3.1. The fitted gradient errors and horizontal BPM gains are compared to the simulation parameters in Fig. 3.2 as examples. The simulation parameters are recovered.

\subsubsection{Simulations with the Booster model}

For the Booster model we start with simpler cases. In one simulation the "measured" ORM is generated by setting all simulation parameters to default values (i.e., 1.0 for gains and 0.0 otherwise) except for the gradient error of one magnet, which is set to $\Delta K_{1}=0.002 \mathrm{~m}^{-2}$, or about $4 \%$ of the nominal gradient. In another simulation all parameters are set to default except that the roll of one magnet is set to $5 \mathrm{mrad}$. Applying the fitting algorithm to the simulated ORM's, although $\chi^{2}$ is brought down 


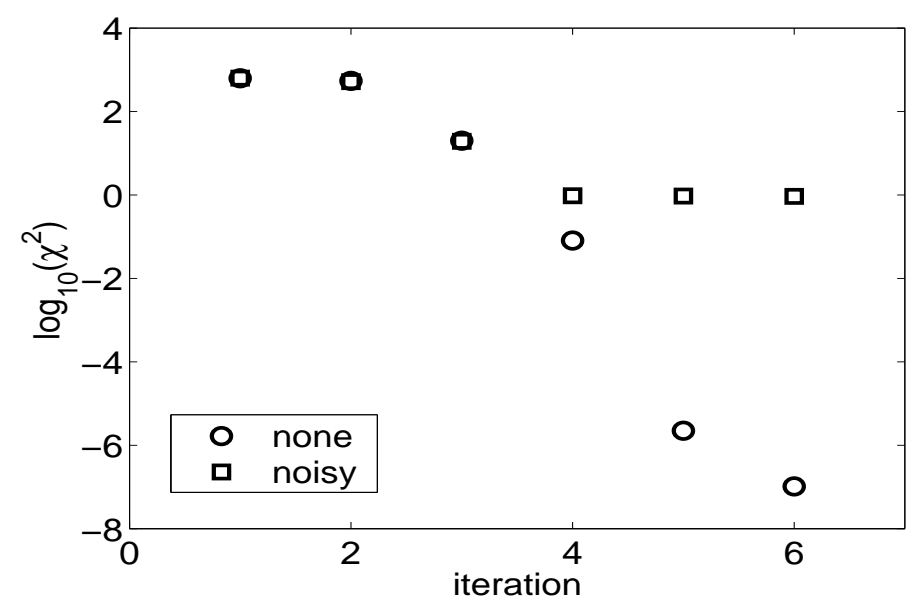

Figure 3.1: The $\chi^{2}$ history for PSR simulation, with ("noisy") or without ("none") random noises.

to practically zero, the solutions don't converge to the expected ones, as shown in Fig. 3.3. Instead, the parameters of nearby magnets pop up and make up part of the contributions to $\chi^{2}$. Such observations clearly suggest correlations between model parameters of the adjacent magnets, i.e., changes of these parameters perturb the ORM in similar patterns. The correlation of parameters can be characterized by the correlation coefficients of the corresponding column vectors in Jacobian matrix $\mathbf{J}$, which are defined as

$$
r=\frac{\mathbf{v}_{\mathbf{1}}^{T} \cdot \mathbf{v}_{\mathbf{2}}}{\left\|\mathbf{v}_{\mathbf{1}}\right\| \cdot\left\|\mathbf{v}_{\mathbf{2}}\right\|},
$$

where $\mathbf{v}_{1}$ and $\mathbf{v}_{2}$ are column vectors of the Jacobian matrix. Fig. 3.4 illustrates the correlation of the adjacent parameters with these coefficients. The gradient errors of neighboring focusing magnets (FD-FU), the rolls of neighboring upstream magnets (FU-DU) or downstream magnets (DD-FD) have strongest correlation.

The correlated parameters are difficult to separate. Their effects over the ORM can cancel each other when they change in certain patterns. For example, increasing 


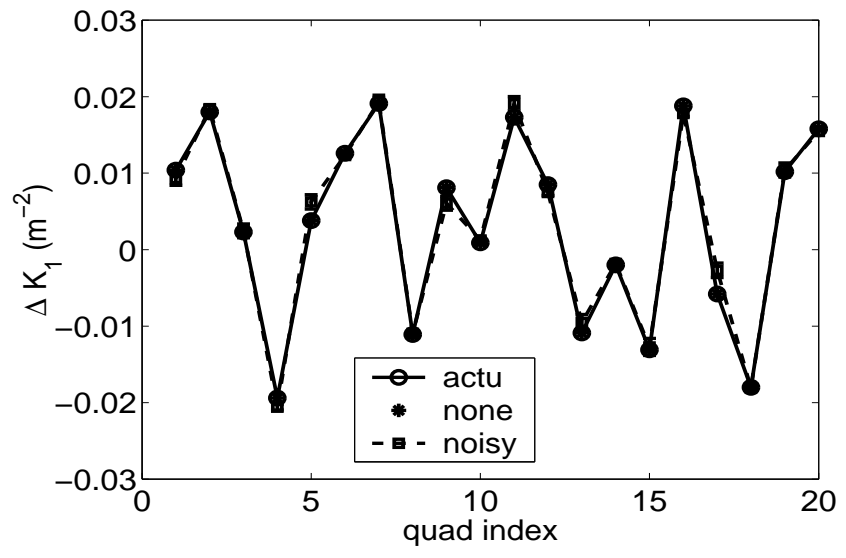

(a) gradients

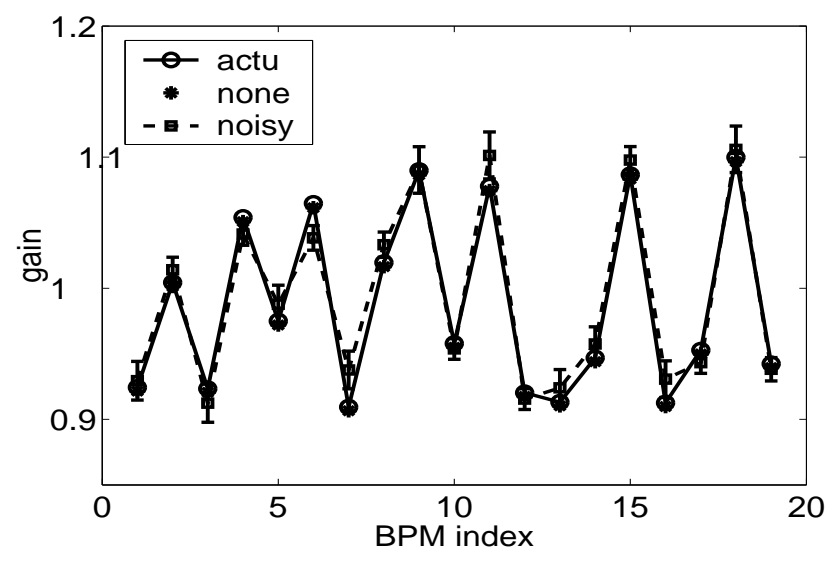

(b) Horizontal BPM gains

Figure 3.2: The fitted parameters of PSR ORM simulations are compared to simulation parameters ("actu") for cases with ("noisy") or without ("none") random noises in the simulated ORM. (a) gradient errors $\left(\Delta K_{1}\right.$ in $\left.\mathrm{m}^{-2}\right)$; (b) horizontal BPM gains. 


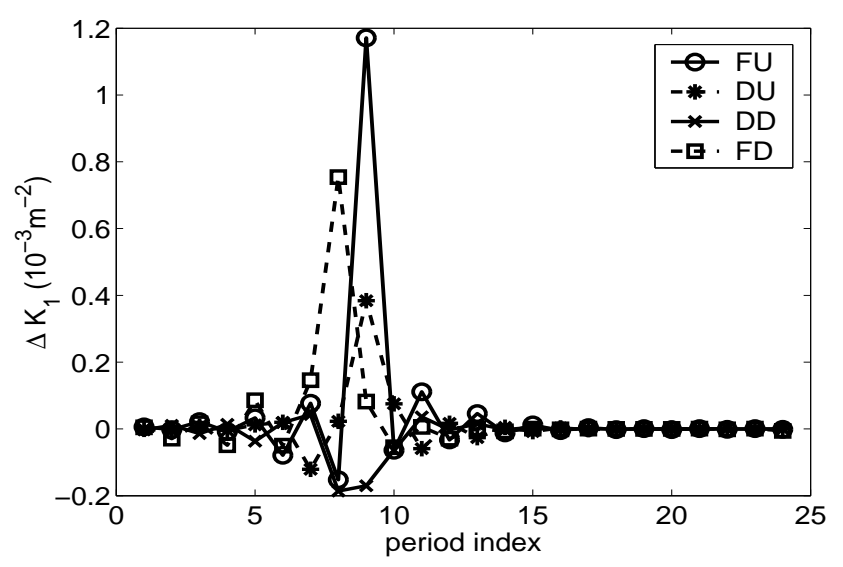

(a)

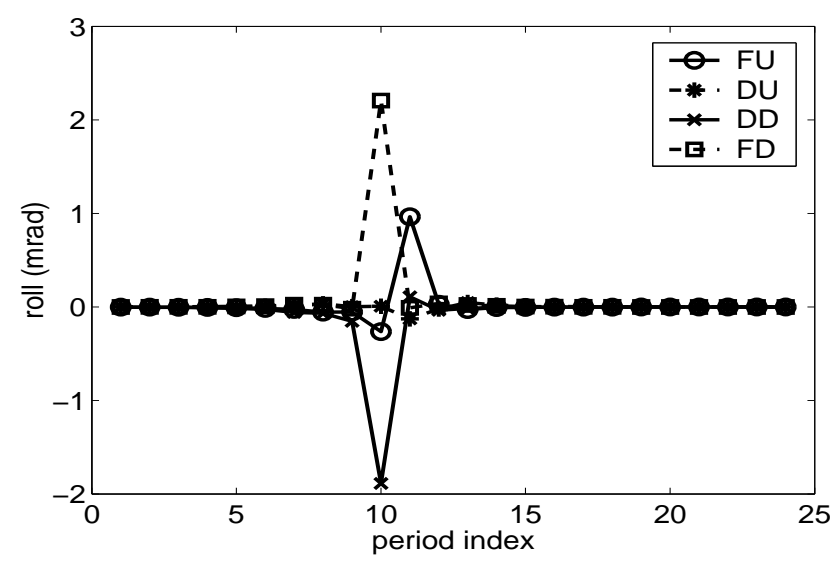

(b)

Figure 3.3: Fitting results for simulations with single model parameter error. (a) The fitted gradient errors for data simulated with $\Delta K_{1}(\mathrm{FU}, 9)=0.002 \mathrm{~m}^{-2}$. The normalized $\chi^{2}$ is reduced from 0.178 to $0.70 \mathrm{E}-5$. (b) The fitted rolls for data simulated with $\operatorname{roll}(\mathrm{FD}, 10)=5 \mathrm{mrad}$. The normalized $\chi^{2}$ is reduced from 0.003 to $0.8 \mathrm{E}-6$. 


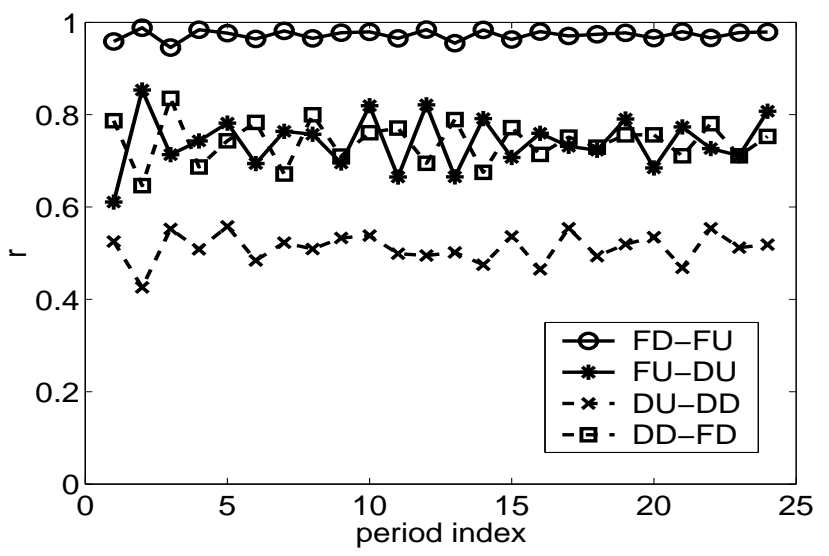

(a) gradients

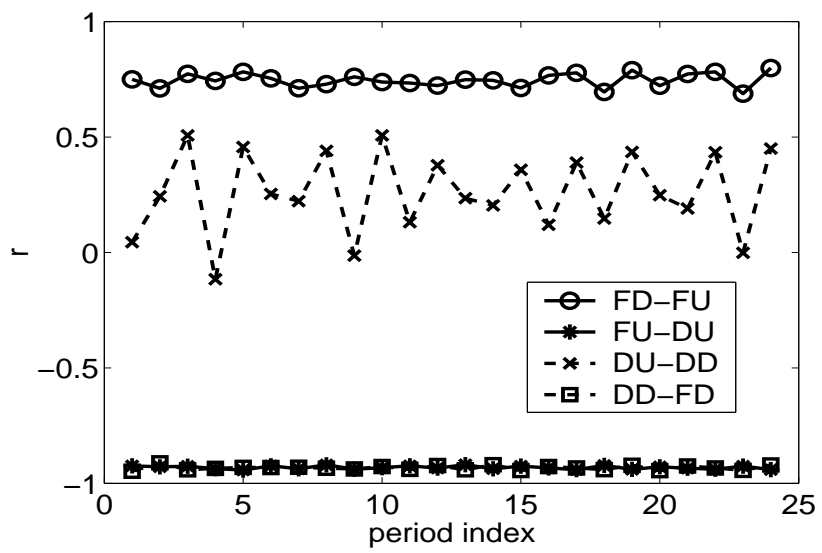

(b) rolls

Figure 3.4: The correlation coefficients for model parameters of neighboring magnets. (a) The gradient error parameters. (b) The roll parameters. 
$\Delta K_{1}$ (the gradient error) of a downstream focusing magnet and decreasing $\Delta K_{1}$ of its neighboring upstream focusing magnet by the same amount results in little change of $\chi^{2}$. These patterns correspond to unconstrained directions of the parameter space by the ORM fitting scheme. The fitting solution would stray along these unconstrained directions to gain even tiny amount of $\chi^{2}$ reduction when there is random noise in the ORM. Fig. 3.5 shows the history of $\sqrt{\chi^{2}}$ vs. the Euclidean norm of model parameters during the iterations for experimental ORM data. Clearly, the solution of the unconstrained fitting scheme drifts around in a large region with little reduction of $\chi^{2}$.

The solution is practically non-unique because it is very sensitive to random noise due to the unconstrained directions. Any vector in the subspace of the parameter space spanned by the unconstrained directions would be an equivalent solution. This causes erroneous behavior of the fitting scheme when it is applied to real data. For example, the gradient errors are as large as $10 \%$ of the nominal value; there are asymmetric patterns between the neighboring parameters; and the error bars of model parameters are huge.

\subsection{The Constrained ORM Fitting Scheme}

Although the individual parameters cannot be completely determined due to correlations, a definitive solution of the ORM problem is still desirable. The solution can be made unique by specifying certain properties of the solution. One choice is to restrain the Euclidean norm of the solution. A better approach is to limit the drifting along the unconstrained directions. The constraints can be imposed by appending additional components to the residual vector. For the "norm" constraints, terms like $\alpha_{i} / \sigma_{\text {type }}$ are added to the residual vector $\mathbf{r}$, where $\sigma_{\text {type }}$ is the characteristic value for the type of parameters. The unconstrained directions are the differences or sums of 


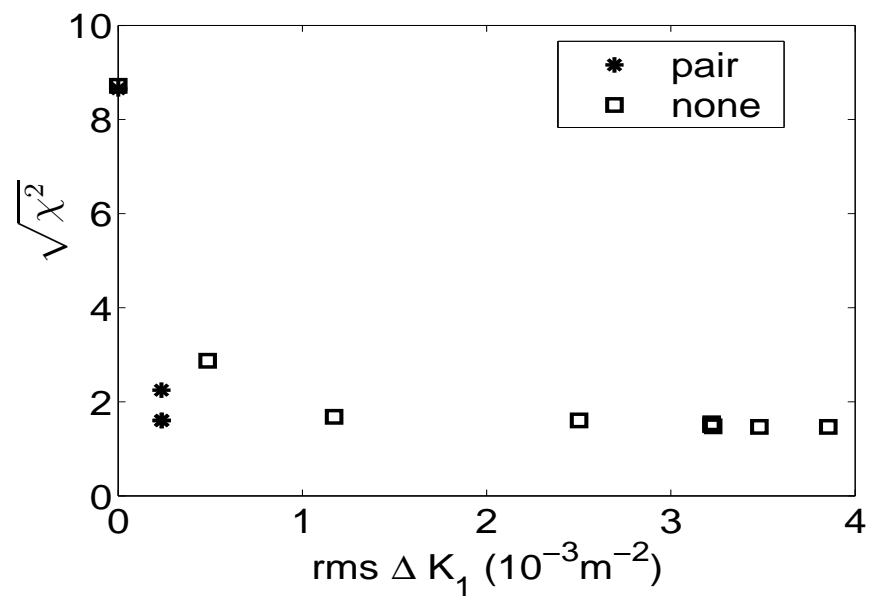

(a) gradient errors

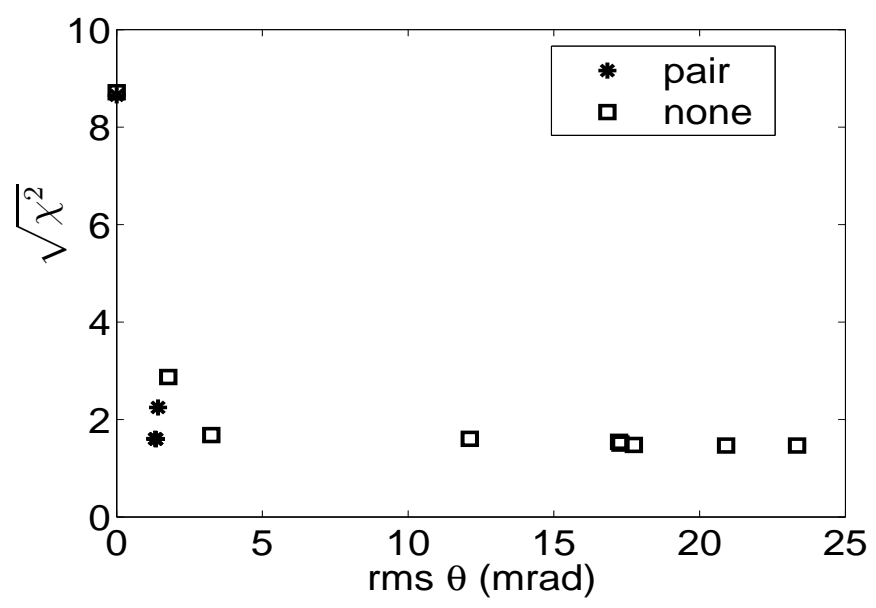

(b) magnet rolls

Figure 3.5: Norm of the residual vector $\left(\sqrt{\chi^{2}}\right)$ vs. norm of the model parameters at each iteration: (a) the norm of gradient errors $\left(\mathrm{m}^{-2}\right)$; (b) the norm of rolls in mrad. The solution is tightly confined with "pair" constraints and is free to drift without constraints ("none"). 
the correlated adjacent model parameters, depending on whether they are correlated positively or negatively. For example, $\Delta K_{1}(\mathrm{FD}, i)$ and $\Delta K_{1}(\mathrm{FU}, i+1)$ are correlated positively, so we can specify to minimize $\Delta K_{1}(\mathrm{FD}, i)-\Delta K_{1}(\mathrm{FU}, i+1)$ by appending $\left(\Delta K_{1}(\mathrm{FD}, i)-\Delta K_{1}(\mathrm{FU}, i+1)\right) / \sigma_{K_{1}}$ to the residual vector. The pairs of parameters required to be minimized include

(a) $2(\theta(\mathrm{FU}, i)+\theta(\mathrm{DU}, i)) / \sigma_{\text {roll }}$, rolls of upstream magnets.

(b) $2(\theta(\mathrm{DD}, i)+\theta(\mathrm{FD}, i)) / \sigma_{\text {roll }}$, rolls of downstream magnets.

(c) $(\theta(\mathrm{FD}, i)-\theta(\mathrm{FU}, i+1)) / \sigma_{\text {roll }}$, rolls of neighboring focusing magnets.

(d) $2\left(\Delta K_{1}(\mathrm{FD}, i)-\Delta K_{1}(\mathrm{FU}, i+1)\right) / \sigma_{K_{1}}$, gradients of neighboring focusing magnets

(e) $\left(\Delta K_{1}(\mathrm{FU}, i)-\Delta K_{1}(\mathrm{DU}, i)\right) / \sigma_{K_{1}}$, gradients of upstream magnets.

(f) $\left(\Delta K_{1}(\mathrm{DD}, i)-\Delta K_{1}(\mathrm{FD}, i)\right) / \sigma_{K_{1}}$, gradients of downstream magnets.

where the additional weighting factor 2 is added to reflect the fact that these parameter pairs are more strongly correlated than the others. These types of constraints are referred as "pair" constraints hereafter. The characteristic values $\sigma_{K_{1}}=0.00055 \mathrm{~m}^{-2}$ and $\sigma_{\text {roll }}=5 \mathrm{mrad}$ are used to scale the parameters for both types of constraints.

A comprehensive simulation is used to study the constrained fitting. The parameters were set to random values within a reasonable range to generate the ORM. The gains were set to within $[0.9,1.1]$, the rolls of BPMs and trims are $[-50,50]$ mrad, the gradient errors are set to $[-0.002,0.002] \mathrm{m}^{-2}$ and the magnet rolls are $[-8,8] \mathrm{mrad}$. The initial normalized $\chi^{2}$ (assuming matrix elements error $\sigma=1.0 \mathrm{~m} / \mathrm{rad}$ in $\chi^{2}$ calculation.) is brought down from 32.1 to below 0.01 for both free or constrained fitting. The correction parameters can be properly recovered. The model parameters don't converge to the expected solution. But they are in good agreement along the constrained directions, as shown in Fig. 3.6. 
The benefit of imposing constraints is a unique solution without loss of information of the ORM. This solution is insensitive to random noise as can be seen in the error bar estimation. Assuming the uncertainty level of each matrix element is $1.0 \mathrm{~mm} / \mathrm{mrad}$, the average error sigma's of gradients and rolls are $\sigma_{\mathrm{K}_{1}}=0.0042 \mathrm{~m}^{-2}$ and $\sigma_{\text {roll }}=$ $78.7 \mathrm{mrad}$ without constraints, $\sigma_{\mathrm{K}_{1}}=2.5 \times 10^{-4} \mathrm{~m}^{-2}$ and $\sigma_{\text {roll }}=1.7 \mathrm{mrad}$ with "pair" constraints. The big error bars of unconstrained fitting come mostly from the unconstrained directions.

\subsection{Application to Experimental Data}

\subsubsection{Measurements of ORM}

A column of the ORM can be measured by changing the current of the corresponding trim dipole to create a "bump" and measuring the changes of beam orbit at all BPMs. Several bump sizes are used and the orbit readings vs. bumps at each BPM are fitted to a linear curve. The slopes are turned to matrix elements according to the specifications of trim dipoles [2]. The relation between the kick angle $\theta$ and the bump $I$ is

$$
\theta=c I=\frac{3000 \times 10^{-6}[\mathrm{~T} \cdot \mathrm{m}]}{3.3357 p[\mathrm{GeV} / \mathrm{c}]} \frac{I}{I_{0}}
$$

where $I_{0}=5.6 \mathrm{~A}$ for a vertical kicker and $I_{0}=3.24 \mathrm{~A}$ for a horizontal kicker. A console program was used to automate the measurements [14].

The uncertainty level of the elements are estimated using the residual $\chi^{2}$ of the linear fittings. The maximum horizontal kick angles are $0.58 \mathrm{mrad}$ for trims at small horizontal beta (short straight sections) and $0.29 \mathrm{mrad}$ for large horizontal beta, resulting in maximum horizontal orbit shifts of $8.0 \mathrm{~mm}$. The maximum vertical kick angles are $0.34 \mathrm{mrad}$ at small vertical beta and $0.17 \mathrm{mrad}$ at large vertical beta with 


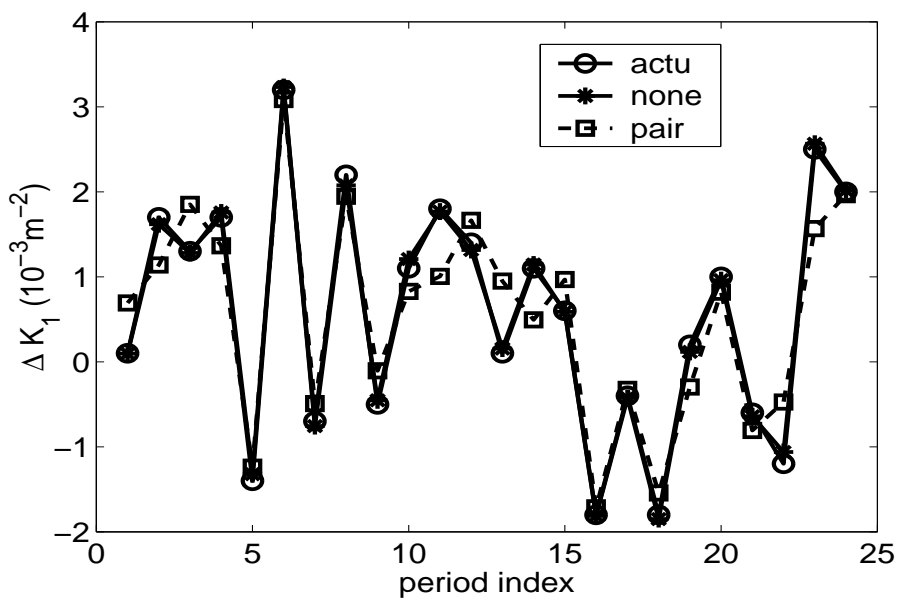

(a) $\Delta K_{1}(\mathrm{FD}, i)+\Delta K_{1}(\mathrm{FU}, i+1)$

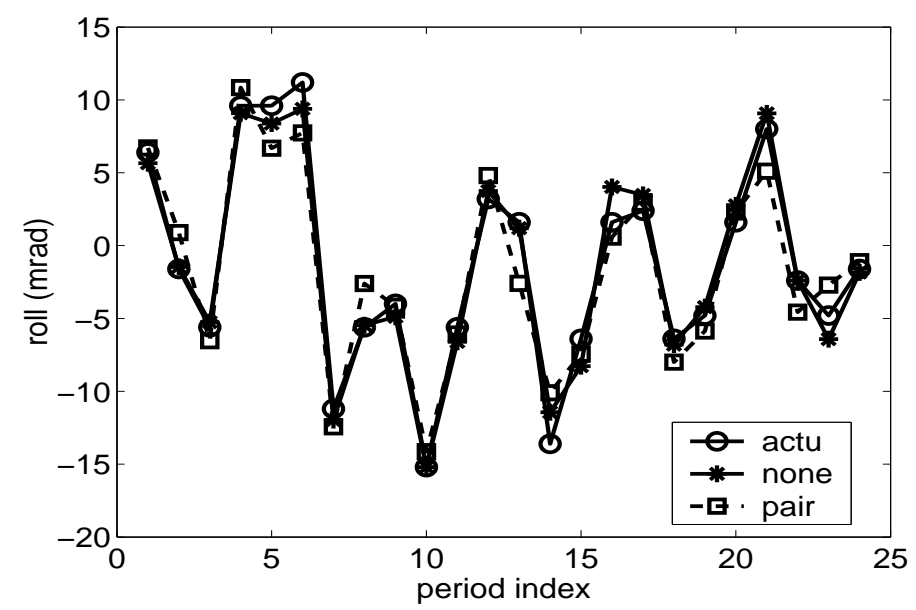

(b) $\operatorname{roll}(\mathrm{FU})-\operatorname{roll}(\mathrm{DU})$

Figure 3.6: Examples of constrained directions of model parameters in the comprehensive simulation. Combinations of simulation parameters ("actu") are compared to the fitting results with ("pair") or without ("none") constraints. 
maximum vertical orbit shift of $3.0 \mathrm{~mm}$. The average error sigma's are $0.33 \mathrm{~m} / \mathrm{rad}$ for $\mathbf{M}_{x x}, 0.54 \mathrm{~m} / \mathrm{rad}$ for $\mathbf{M}_{x z}, 0.07 \mathrm{~m} / \mathrm{rad}$ for $\mathbf{M}_{z x}$ and $0.15 \mathrm{~m} / \mathrm{rad}$ for $\mathbf{M}_{z z}$. The vertical blocks have better precision because the vertical orbit has less cycle-to-cycle variations than the horizontal orbit. The vertical BPM data have more weight in the fitting problem. When the ORM data were taken, two vertical BPMs (VL10 and VL13) and one vertical kicker (VS05) were malfunctioning.

The betatron tunes are measured by BPM turn-by-turn data taken while the beam is pinged. The dispersion functions are also measured by changing the radial reference position (i.e., ROF curve). The ROF curve, and hence $\Delta p / p$ is changed by several different levels. The resulting horizontal orbit offsets are fitted to $\Delta p / p$ to linear curves to obtain dispersion at each BPM along with its error estimation. The betatron tunes and dispersion data are included in the fitting by extending the residual vector. The dispersion terms are $\left(D_{i}^{\text {meas }}-D_{i}^{\text {model }}\right) /\left(b_{h, i} \sigma_{D, i}\right)$, where $b_{h, i}$ is horizontal BPM gain and $\sigma_{D, i}$ is error sigma for dispersion measurements. The inclusion of dispersion function would de-couple the BPM gains and kicker gains [8].

The settings of the trim quadrupoles and skew quadrupoles of the correctors were recorded and are used to construct the Booster lattice model as the basis model. The ORM data are taken at several different time points in the cycle. Each point is called a frame. Information of the data frames early in the cycle are shown in Table 3.2. The dispersion was measured at $1.0 \mathrm{~ms}$ after injection, corresponding to data frame 1.

\subsubsection{The fitting results}

The results presented below are for data frame 1 with dispersion data. Fig. 3.5 shows

the history of $\sqrt{\chi^{2}}$ vs. the norms of model parameters during the iterations. We see that the constrained solution converges quickly while the unconstrained solution 
Table 3.1: The $\chi^{2}$ contributions

\begin{tabular}{l|c}
\hline H BPM gains & 1.8 \\
V BPM gains & 11.8 \\
H trim gains & 0.15 \\
V trim gains & 0.6 \\
BPM rolls & 0.9 \\
H trim rolls & 0.4 \\
V trim rolls & 3.5 \\
dp/p & 18.6 \\
gradients & 3.8 \\
rolls & 24.2 \\
\hline
\end{tabular}

strays around with little reduction of $\chi^{2}$. The normalized $\chi^{2}$ is 76.0 initially and is reduced to 2.5 by constrained fitting.

The contribution to $\chi^{2}$ of any type of parameters is evaluated by setting all other types of parameters to their fitted values except for the type which is set to the default. The contributions are listed in Table 3.1. We notice the major contributors are magnet rolls, kick-induced momentum deviation, vertical BPM gains and the gradient errors.

As indicated by the normalized $\chi^{2}$, the model ORM and dispersion functions show good agreement with their measured counterparts through the fitting process. Comparisons are made in Fig. 3.7 and 3.8.

The horizontal bumps cause big changes of momentum deviation because of the Booster's radial orbit control mechanism which always tries to minimize the difference between the horizontal orbit at section L20 and the reference value (ROF curve). If the feedback system works perfectly with instant response time, the beam orbit at 


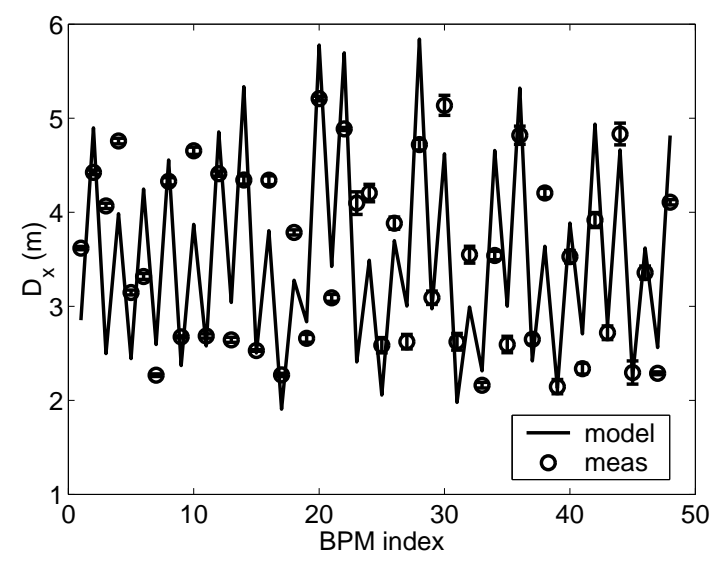

(a) before

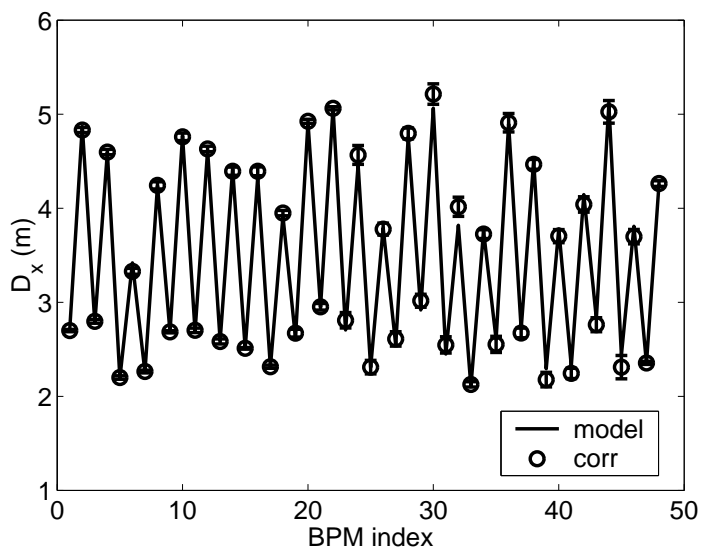

(b) after

Figure 3.7: Comparison of dispersion functions before and after the fitting. Note that the dispersion functions are corrected by the fitted horizontal BPM gains after the fitting.

L20 would be fixed, i.e.,

$$
D_{x}(\mathrm{~L} 20)\left(\frac{\Delta p}{p}\right)_{j}+M_{x x}(\mathrm{~L} 20, j) \theta=0
$$

is satisfied. Hence the momentum deviation due to unit kick angle of the $j$ 'th trim is expected to be $-M_{x x}(\mathrm{~L} 20, j) / D_{x}(\mathrm{~L} 20)$. The comparison is shown in Fig. 3.9. Although the orbit at L20 is not fixed, the overall effect is consistent with the picture described above.

The correction parameters (Fig. 3.10) found by fitting can be used to calibrate the BPMs or trims. There were several BPMs which had unexpected large gains with values near 1.5. It was then found the mis-calibration of these BPMs was due to a 

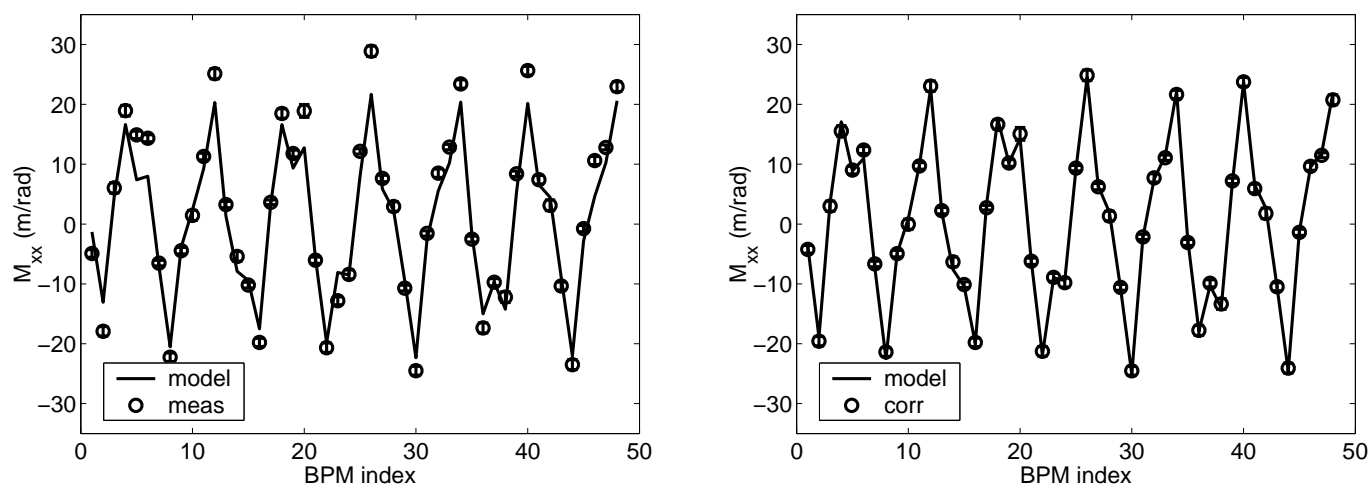

(a) before

(b) after
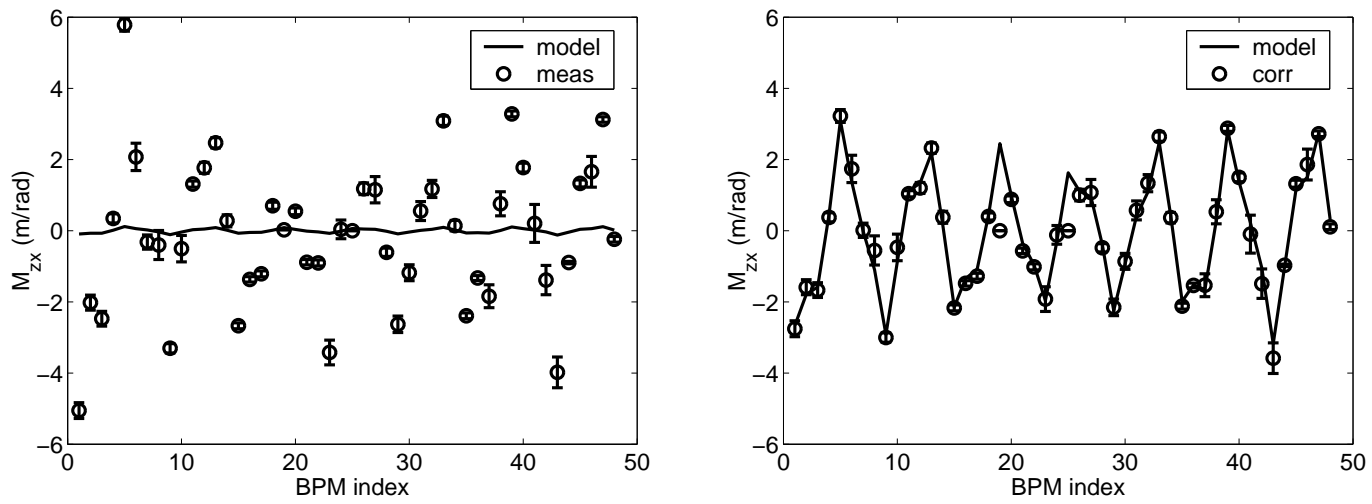

(c) before

(d) after

Figure 3.8: Comparisons of model and measured orbit response for horizontal trim S01, before and after fitting. (a)(b) Horizontal orbit response; (c)(d) Vertical orbit response. 


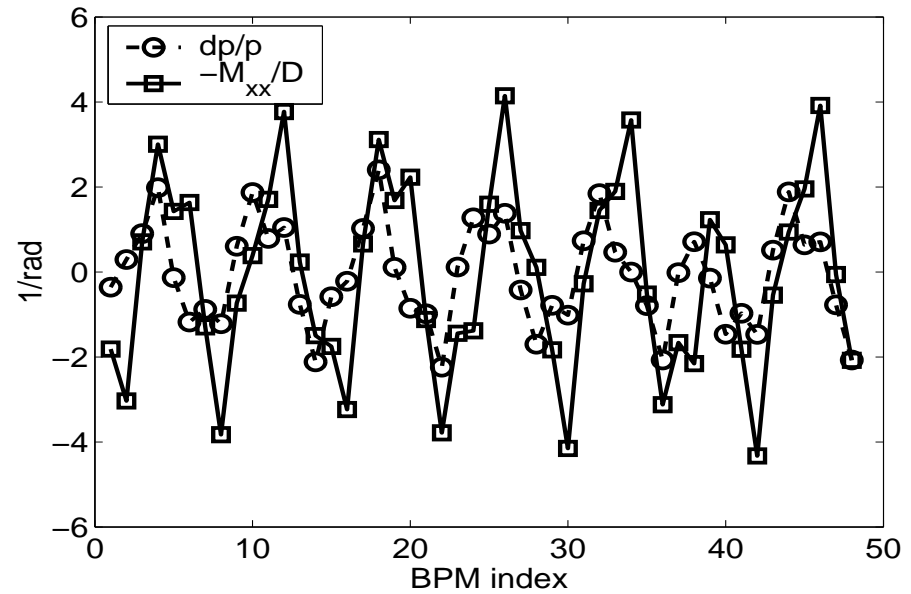

Figure 3.9: The fitted kick-induced momentum deviation per unit kick angle (circle) is compared to the orbit response at section L20 (square). The agreement of the two curves indicates the behavior of the radial position feedback loop. 
bug $^{2}$ in the BPM calibration routine [15]. These large gains disappeared when the bug was fixed. There were two horizontal trims with weaker gains near 0.8 . The other BPM and trim gains had rms deviations of $5 \%$ around their average values. The rolls of BPMs were found to be within $1.5 \mathrm{deg}$. The rolls of kickers were within $2 \mathrm{deg}$.

The fitting solution provides us with a model that is equivalent to the real Booster in the sense that it gives the same orbit response properties. Since the ORM is essentially specified by beta functions and betatron phase advances, the fitted model should produce the same lattice functions as the real Booster. In other words, we have derived the beta functions and phase advances from the measured ORM. Such information can be used in machine operations, e.g., to find suitable ratios of trim strengths to create local orbit bumps.

Besides the lattice functions, the fitted model also provides us with direct information about magnet imperfections. As we have seen in simulations, some combinations of the model parameters, such as the gradient error sums of adjacent focusing magnets, are well constrained by the fitting scheme. These combinations in the fitting solution should approximate that of the real Booster. Some constrained combinations of the model parameters are shown in Fig. 3.11. The average gradient errors of the focusing magnet in most periods are below $0.0004 \mathrm{~m}^{-2}$, i.e., $0.8 \%$ of the nominal values. Such deviations are within the design tolerance 1\% [2]. The relative rolls of the upstream or downstream magnets are below 6 mrad.

Uncoupled fitting is also conducted in which the off-diagonal blocks of the ORM and all roll parameters are excluded. This simpler fitting scheme found the same solution for relevant parameters, i.e., the gains, kick-induced momentum deviation and gradient errors. This is a reasonable result because the roll parameters are usually small so that their effects on the diagonal (uncoupled) blocks are second order, while

\footnotetext{
${ }^{2}$ These turned out to be modified BPM's for which the calibration data had not been set up.
} 


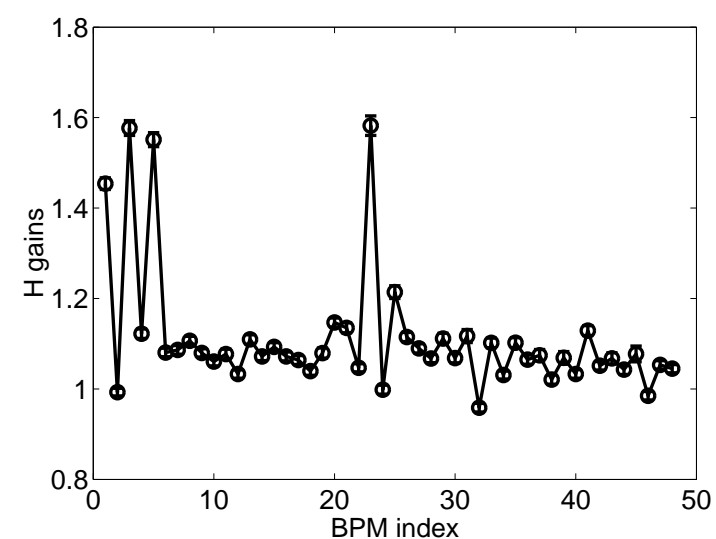

(a) H BPM

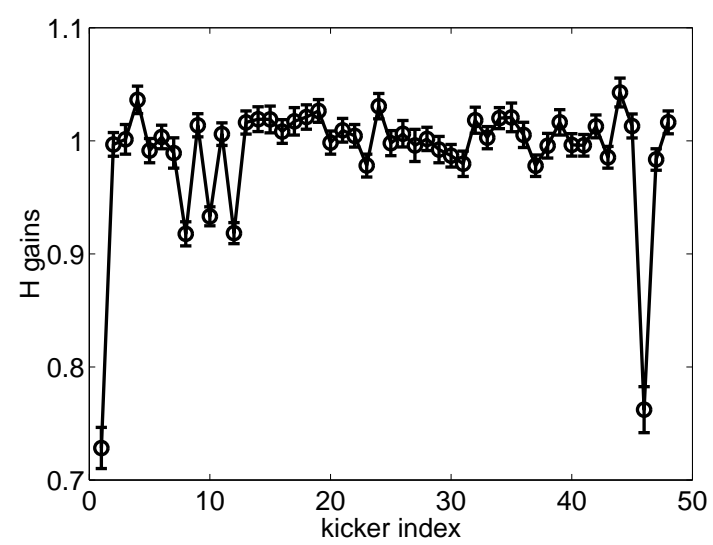

(c) H kicker

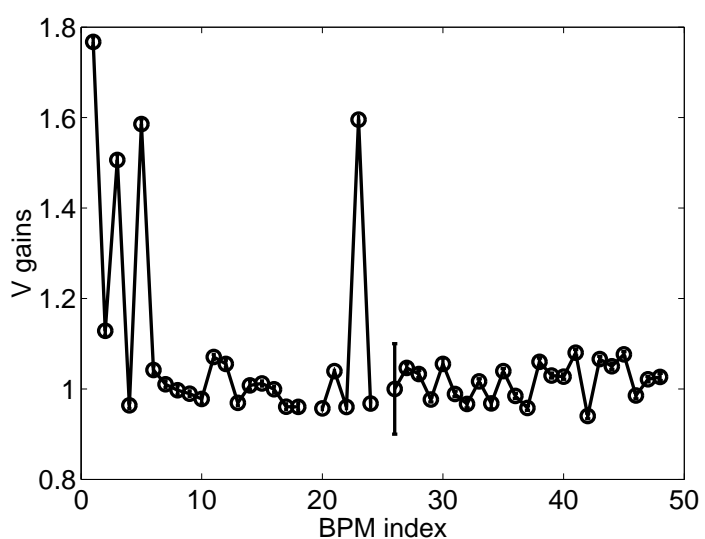

(b) V BPM

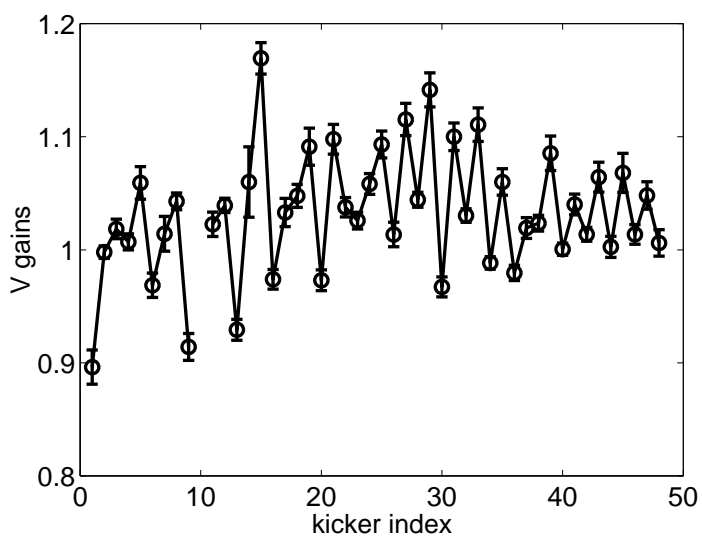

(d) V kicker

Figure 3.10: The fitted BPM and kicker gains. The large BPM gains in (a)(b) were found to be due to a bug in the calibration routine which had disappeared when the bug was fixed. The horizontal kicker gain at L01 (index 1) was known to be weaker than the others. 


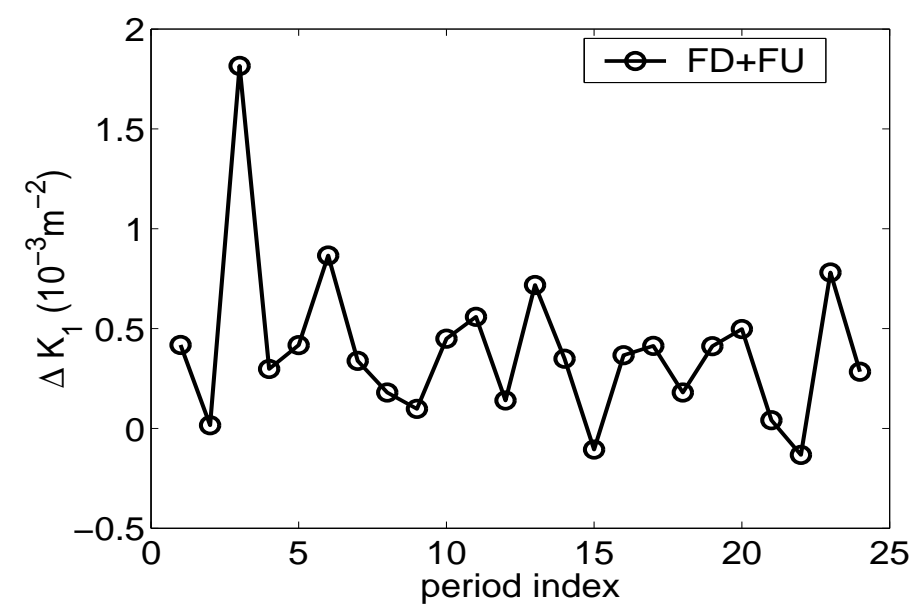

(a) gradients

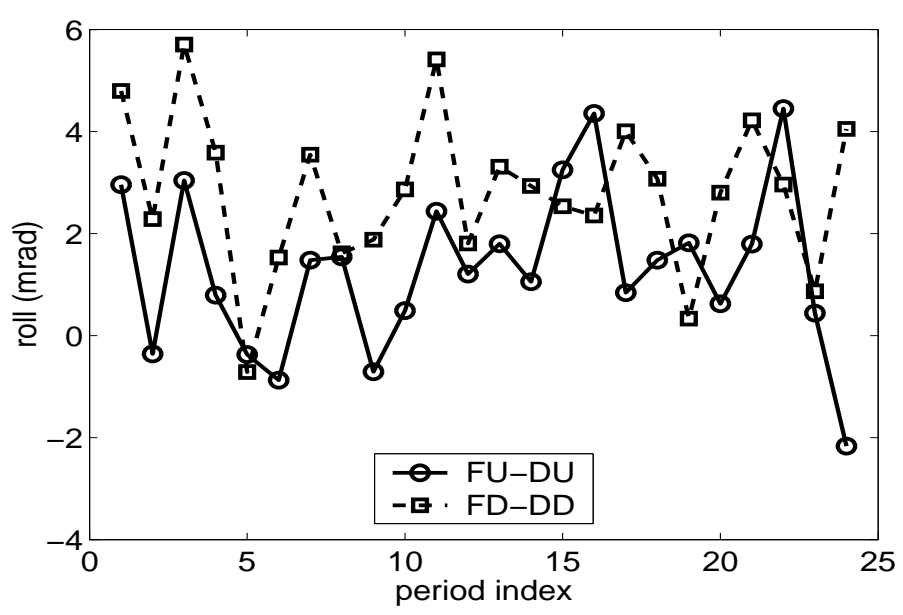

(b) rolls

Figure 3.11: Constrained combinations of the fitted model parameters. (a) Sum of the gradient errors of adjacent focusing magnets. (b) Relative rolls of the upstream magnets (FU-DU) and the downstream magnets (FDDD). 
Table 3.2: The ORM data frames and corresponding $\chi^{2}$

\begin{tabular}{llllll}
\hline frame & time $(\mathrm{ms})$ & $E_{k}(\mathrm{GeV})$ & $P_{0}(\mathrm{GeV} / \mathrm{c})$ & initial $\chi^{2}$ & final $\chi^{2}$ \\
\hline 1 & 0.9 & 0.41 & 0.97 & 71.9 & 2.7 \\
2 & 1.8 & 0.43 & 1.00 & 90.0 & 3.3 \\
3 & 2.7 & 0.47 & 1.05 & 94.5 & 3.3 \\
4 & 3.5 & 0.52 & 1.11 & 101.9 & 3.4 \\
\hline
\end{tabular}

their effects on the off-diagonal (coupled) blocks are first order.

We compared the fitting results for the four data sets (frames) in the Booster cycle. Table 3.2 shows information of the data frames and the initial and final normalized $\chi^{2}$ of the fittings. The correction parameters obtained from the 4 data frames are consistent. However the model parameters are different in general. It is noticed that the combined gradient errors of neighboring focusing magnets are correlated with the horizontal beam orbit.

The horizontal orbit of the Booster drifts during the cycle because the trim dipoles are fixed while the beam energy is ramped up. The horizontal orbit changed between the time when ORM data of different frames were taken. Since the sums of gradient errors of adjacent focusing magnets are contrained, we use them to study the correlation between the gradient errors and the orbit drifts.

The stable horizontal orbit $x_{0}$ at each BPM is obtained in the linear fitting of orbits vs. bumps. Its standard deviation $\sigma_{x_{0}}$ is used as error estimation, which represents the cycle-to-cycle orbit stability. It is found that on average $\sigma_{x_{0}}=0.11$ $\mathrm{mm}$ in LONG sections and $\sigma_{x_{0}}=0.17 \mathrm{~mm}$ in SHORT sections. The same constrained fitting scheme (not including dispersion data) is applied to four data frames early in the cycle.

We consider the SHORT sections that have large changes of horizontal orbit 
( $\geq 1 \mathrm{~mm})$. The combinations of gradient errors $\Delta K_{1}(\mathrm{FD})+\Delta K_{1}(\mathrm{FU})$ are plotted against the horizontal orbit changes $\Delta x_{0}$ in Fig. 3.12 for some sections. The linear correlation between them is clearly indicated. The linear relation between gradient errors and horizontal orbits implies that the source of gradient errors is the sextupole components, which contributes to quadrupole gradient through

$$
\Delta K_{1}=K_{2} \Delta x_{0}
$$

where $K_{2}$ is the normalized sextupole coefficient.

\subsection{Summary}

In this study we measured the fully-coupled orbit response matrix of the Fermilab Booster near injection and fit it to the lattice model. We found that the model parameters of the adjacent magnets are strongly correlated, which prevents the full determination of all individual model parameters. The fitting problem does not have a unique solution with standard ORM approach. By imposing constraints to remove the ambiguity of the unconstrained directions, we have obtained an unique, robust solution which determines all correction parameters and an equivalent lattice model of the Booster. The solution makes consistent predictions of the constrained combinations of the model parameters. By studying the linear correlation of fitted gradient errors and the changes of horizontal orbit, we have shown that the source of the gradient errors are orbit offsets in sextupole fields of the combined-function magnets. The study also confirms that the gradient errors of the focusing magnets are within design limits. 


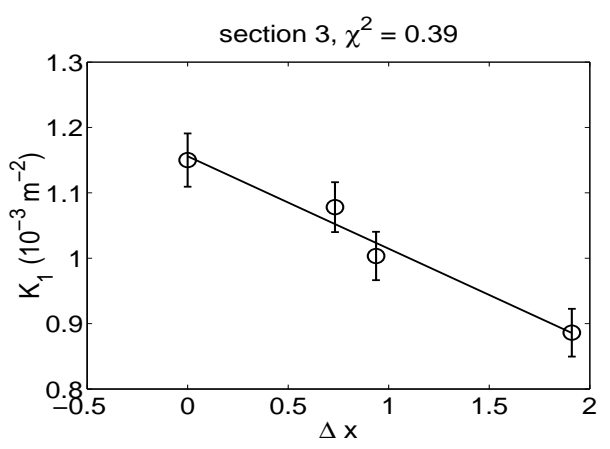

(a) S03

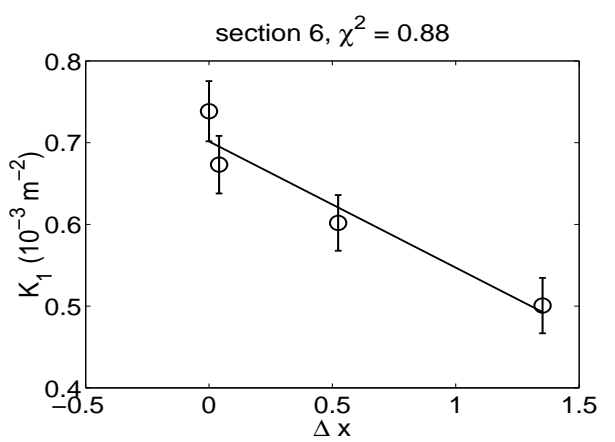

(c) S06

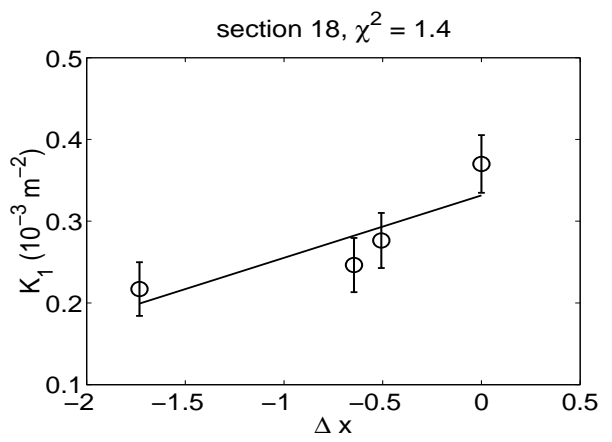

(e) S18

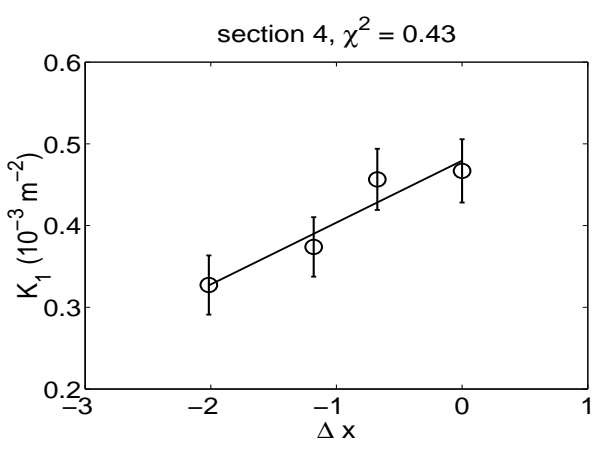

(b) S04

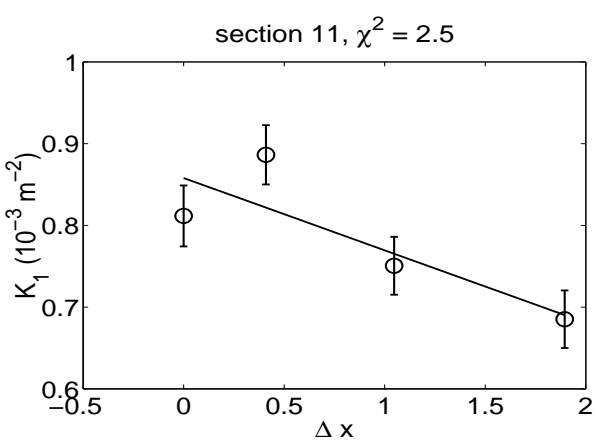

(d) S11

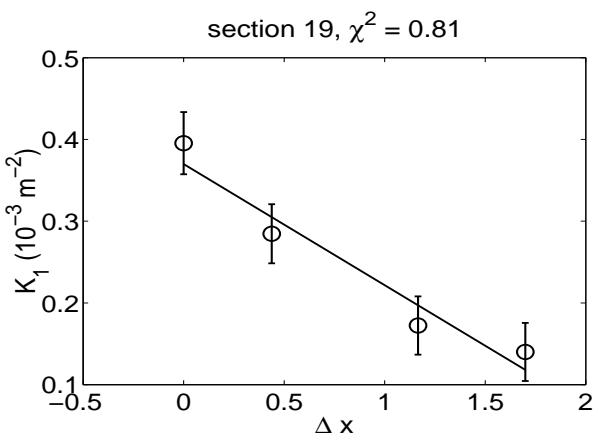

(f) S19

Figure 3.12: Examples of linear fitting curves of $\Delta K_{1}\left(10^{-3} \mathrm{~m}^{-2}\right)$ vs. $\Delta x_{0}(\mathrm{~mm})$ at locations with large changes of the horizontal orbit. The linear correlation indicates sextupole focusing. 


\section{Chapter 4}

\section{Independent Component Analysis for BPM Data Analysis}

Beam position monitors (BPMs) detect the transverse motion of the beam. There are usually many BPMs around the synchrotron ring, with one or more in a period. Since the BPMs of many accelerators are able to digitize and record their readings on a turn-by-turn basis, they can provide massive data about the evolution of the beam. The beam transverse motion is influenced by many physical factors. The multiple BPM turn-by-turn data contain much information about the underlying factors which affect the performance of the machine. However, it is important to have the right tool to uncover this information.

The data sampled by BPMs reflect the beam transverse motion, which is a combination of betatron motion, synchrotron motion (coupled through dispersion) and perturbations from other sources, such as noise, ground motion, vibrations, wake fields, etc. If the BPM system is linear, the sampled data can be considered as a linear mixture of a few physical source signals. These signals are independent of each other if they originate from different physical processes. The ultimate goal of data 
analysis is to uncover these independent source signals.

The model independent analysis (MIA) is a method to analyze multiple BPM turn-by-turn data of synchrotrons or pulse-by-pulse data for linacs. It is aimed at untangling the eigenmodes [16] with statistical methods and then use the resulting spatial and temporal functions of the modes to identify betatron motion and other sources of perturbations. The MIA method has been applied to study linac transport systems and beam dynamics in high brightness storage rings $[17,18]$.

MIA is essentially a principal component analysis (PCA), which tries to find a linear transformation of the samples to uncover the maximum amount of variance in the least number of uncorrelated components. When these source modes are weakly coupled or non-degenerate (unequal eigenvalues), MIA can properly isolate these independent modes. When eigenmodes are coupled, one needs to apply a narrowband filtering to isolated the relevant modes [18].

For high intensity rapid cycling accelerators, the source modes are normally found to be strongly coupled. For example, the data obtained from the Fermilab Booster show that the synchrotron motion is strongly coupled to the betatron modes. The contaminated signal data should be removed by averaging before the PCA analysis can be properly carried out. Other strong perturbing signals (e.g. from a bad BPM) can severely degrade the betatron modes.

The independent component analysis (ICA) method provides a remedy for MIA's limitations by identifying the independent source signals from the samples using unequal time correlations. Once identified, the source signals can provide information on the betatron and synchrotron motion and other perturbation modes according to their spatial and temporal functions. This new method is more immune to modemixing and noise. Because of its ability to isolate modes, the ICA can be used to identify and study the unknown beam motions due to various perturbing sources. Implementation of the ICA algorithms depends on the nature of the source signals 
$[21,23,22,26]$. The time-correlation based method is particularly efficient in isolating narrowband source modes and is therefore used in this study.

This chapter intends to establish the theoretical basis of the independent component analysis for beam diagnosis. It is organized as follows. The basics of PCA-based MIA and its limitations are given in section 4.1. The principles of ICA and the application of ICA to turn-by-turn BPM data analysis are presented in section 4.2. Section 4.3 covers simulation studies which compare MIA and ICA and show the advantages and limitations of this new method. In section 4.4 a new approach of lattice modeling using the measured lattice functions is discussed.

\subsection{The Basics of PCA-based MIA}

MIA considers the data sampled by BPMs as combinations of components that are driven by separate physical variables and it tries to isolate these components (or modes) to facilitate the study of the underlying physical variables. The separation of the modes is based on the fact that there is much redundancy in the data sampled by many BPMs at different locations. In fact, the number of significant modes is usually smaller than the number of BPMs. Making use of statistical characteristics of the data sample, it is possible to find an interpretation of the data with fewer number of orthogonal components. These components are treated as real physical variables. MIA does not assume a model of the underlying physical variables or the components beforehand. Instead, it explores the second-order statistics information with singular value decomposition (SVD).

Let the BPM readings of $N$ turns or pulses from $m$ BPMs be put into a matrix 
form as

$$
\mathbf{X}=\left(\begin{array}{cccc}
x_{1}(1) & x_{1}(2) & \ldots & x_{1}(N) \\
x_{2}(1) & x_{2}(2) & \ldots & x_{2}(N) \\
\vdots & \vdots & \ddots & \vdots \\
x_{m}(1) & x_{m}(2) & \ldots & x_{m}(N)
\end{array}\right)
$$

Each row of the matrix is made "zero mean" by subtracting the average of the row. Apply SVD to matrix $X$ to obtain

$$
\begin{aligned}
\mathbf{X}_{m \times N} & =\mathbf{U}_{m \times m} \mathbf{S}_{m \times N} \mathbf{V}_{N \times N}^{T} \\
& =\sum_{i}^{m} s_{i} \mathbf{u}_{i} \mathbf{v}_{i}^{T}
\end{aligned}
$$

with orthogonal matrices $\mathbf{U}=\left(\mathbf{u}_{1}, \mathbf{u}_{2}, \cdots, \mathbf{u}_{m}\right), \mathbf{V}=\left(\mathbf{v}_{1}, \mathbf{v}_{2}, \cdots, \mathbf{v}_{N}\right)$ and diagonal matrix $\mathbf{S}$ with diagonal elements $s_{i}$. The column vectors of $\mathbf{U}$ and $\mathbf{V}$ are called spatial patterns and temporal patterns of the modes, respectively.

In the case of synchrotron BPM turn-by-turn data, the main component is the coherent betatron motion of the beam centroid if it is excited. It has been proven that if the BPM data are composed of betatron motion and random noises, the results of SVD are two non-trivial modes representing betatron motion and the other modes are from noises [16]. The two betatron modes are orthogonal and their spatial patterns can be used to derive the beta function and betatron phase advance. This feature has made MIA a convenient method to measure beta function and phase advance [17]. By throwing away the noise modes, one can re-construct the BPM data with reduced noise level.

The SVD-based MIA is a principal component analysis (PCA) method. Suppose the multiple-BPM sample of one pulse or one turn denote a point in an $m$-dimensional space, then the whole data set corresponds to $N$ points in this space which form an ellipsoid-like distribution. MIA obtains the principal axis of this distribution via SVD. The principal axes form an orthogonal basis of the data space. The components are 
ordered by their strengths which correspond to the singular values. The betatron motion-dominated distribution is a $2 \mathrm{D}$ plane in the $m$-dimensional space.

When the strengths (e.g., the eigen-values) of two or more components are nearly equal, these components tend to be mixed by the PCA method because any combination of the involved components would also have nearly equal strength and can serve as a principal component. For physical factors that are weaker than the betatron motion, they often contribute variances of the same order of magnitude and can easily be mixed by the PCA-based MIA. The betatron modes are often mixed with other modes if, for example, there are strong synchrotron motion or bad BPMs with high noises. Thus it is absolutely necessary to pick out the bad or noisy BPMs and the low-frequency components have to be filtered out before MIA can obtain pure betatron modes in a fast-ramping synchrotron like the Booster. MIA faces difficulties even with betatron motion alone if there is linear coupling. The mixing of two tune signals degrade the result of beta function measurement and make it difficult to measure the betatron phase advance.

These limitations of PCA-based MIA prevent it from gaining the full power of mode separation. The ICA method, however, can overcome these limitations by introducing advanced methods that use information beyond plain second-order statistics. The MIA and ICA methods will be compared and contrasted in the next sections.

\subsection{ICA for Synchrotron Beam Diagnosis}

In this section we will introduce the application of an independent component analysis method which is based on the time correlation of the signals to extract the important driving sources and less-important perturbation sources from BPM turn-by-turn data taken around synchrotrons. Compared to the PCA-based MIA, it has better ability to separate and identify the underlying physical variables. 
A brief introduction to the general ICA is given first, followed by details of the time-correlation based method which is efficient for BPM data. Then we will show how the latter can be applied to BPM turn-by-turn data to separate betatron motion, synchrotron motion and other possible perturbation sources. The linear lattice functions including betatron amplitude function, betatron phase advance and dispersion function are measured from the separated modes. Simulation studies which demonstrate the advantages of this method will be presented in the next section.

\subsubsection{Principles of independent component analysis}

The independent component analysis (ICA) is a relatively new data analysis technique whose goal is to uncover the underlying random variables or source signals from multivariate samples. It considers the samples as linear mixtures of the variables or sources. In achieving the goal of source separation, it does not assume any specific knowledge of how these variables or sources are mixed in the samples other than that they are mutually independent. The "cocktail-party" problem is a perfect example to illustrate the situation. Consider $n$ people speaking simultaneously in a party and their voices are picked up by $m$ microphones distributed around the room. The signal recorded by each microphone is a linear mixture of the voices (sources) depending on the distances and directions of the sources. Let $x_{i}, i=1,2, \cdots, m$ be the samples of microphones and $s_{j}, j=1,2, \cdots, n$ be source signals, then

$$
\left(\begin{array}{c}
x_{1} \\
x_{2} \\
\vdots \\
x_{m}
\end{array}\right)=\mathbf{A}_{m \times n}\left(\begin{array}{c}
s_{1} \\
s_{2} \\
\vdots \\
s_{n}
\end{array}\right)
$$

where $\mathbf{A}$ is a matrix with constant elements called the mixing matrix. The ICA method intends to isolate the sources $s_{j}$ from the sample signals $x_{i}$ without considering 
the positions of the people or microphones. This is often referred as the blind source separation (BSS) problem. In a more general sense, $\mathbf{x}$ and $\mathbf{s}$ need not be signals. They can be just random variables.

The ICA is the same as the PCA in that both methods try to find an interpretation of the samples which helps the understanding of the physical causes. The PCA method is based on the uncorrelatedness of the latent random variables (or source signals). Or equivalently it seeks an orthogonal basis such that the covariance matrix is diagonal. If the latent variables are white (i.e., no time correlation) and gaussian, uncorrelatedness is equivalent to statistical independence. In this case, the PCA does the best one can do and there is no additional information to identify the latent variables. However, if the random variables have more features or are more structured than white gaussian noise, the ICA method can uncover the original variables or sources.

The ICA techniques fall into three categories according to what features of the sources are used [23]. The first category assumes the sources are non-gaussian. Since the independent variables are nonlinearly uncorrelated, meaning that the nonlinear functions $g\left(s_{1}\right)$ and $h\left(s_{2}\right)$ of two independent variable $s_{1}$ and $s_{2}$ are uncorrelated, the independent variables can be found by seeking a transformation of the samples such that the resulting components are nonlinearly uncorrelated with properly chosen nonlinear functions. On the hand, according to the central limit theorem, the sums of non-gaussian variables are statistically more gaussian, the independent components thus have maximum non-gaussianity. The independent components can be obtained by transforming the samples to maximize the non-gaussianity of the resulting components with a proper measure of the non-gaussianity. The ICA algorithms in this category include Joint Approximate Diagonalization of Eigen-matrices (JADE) [32], FastICA [33], etc.

The second category makes use of time correlations of the source signals. The 
source signals are assumed to have narrow-band power spectra and their spectra are non-overlapping. Consequently the time-lag covariance matrices are diagonal. The algorithms include AMUSE [25], Second Order Blind Identification (SOBI) [26], etc. Since the BPM signals generally satisfy the conditions, these methods are most efficient for BPM turn-by-turn data analysis. The SOBI algorithm is more robust because of the use of multiple time-lag covariance matrices and is therefore our choice. The details of SOBI algorithm will be given later in this section.

The third category assumes the source signals are non-stationary which means their variances are time-varying. By examining the covariance matrices of the samples in different time windows, the independent components can be found [27, 22]. There are also algorithms that combine the advantage of the second and third categories, e.g., Second-Order Nonstationary source Separation (SEONS) [29].

\subsubsection{The time-correlation based ICA and its application to BPM data}

The time correlation-based ICA method assumes that the source signals have nonoverlapping power spectra, which is usually true for BPM turn-by-turn signals because the source signals are often harmonic oscillations with different tunes. The assumption assures that the un-equal time correlation matrices of the source signals are diagonal. The de-mixing matrix, which transform the sample data vector to the source signal vector is found as the joint diagonalizer of the un-equal time correlation matrices of the sample data with selected time-lag constants. Since the source modes can be isolated, their origin can be identified. Some of these modes are horizontal and vertical betatron oscillations and synchrotron oscillation. These modes enable us to study not only the betatron motions but also the linear coupling and synchrotron motion from BPM data. 
The linear response of a dynamical system is represented by the relation between the $m$-dimensional observation vector $\mathbf{X}(t)$ and the $n$-dimensional source signal vector $\mathbf{s}(t)$ by

$$
\mathbf{X}(t)=\mathbf{A s}(t)+\mathcal{N}(t),
$$

where $\mathbf{A} \in \Re^{m \times n}$ is the mixing matrix with $m \geq n$ ( $n$ is unknown $\grave{a}$ priori) and $\mathcal{N}(t)$ is the noise vector. The noises are assumed to be stationary, zero mean, temporally white and statistically independent of the source signal $\mathbf{s}(t)$. The task is to determine both the mixing matrix $\mathbf{A}$ and the source signals $\mathbf{s}(t)$ from the sample signal $\mathbf{x}(t)$.

The source signals are assumed to be mutually independent and temporally correlated. Consequently the time-lagged covariance matrix $\mathbf{C}_{\mathbf{s}}(\tau) \equiv\left\langle\mathbf{s}(t) \mathbf{s}(t+\tau)^{T}\right\rangle$ is diagonal, i.e. $\left\langle\mathbf{s}_{\mathbf{i}}(t) \mathbf{s}_{\mathbf{j}}(t+\tau)^{T}\right\rangle=S_{i}(\tau) \delta_{i j}$. Here $\langle\cdots\rangle$ stands for mathematical expectation, $\delta_{i j}$ is Kronecker delta and $\tau$ is the time-lag constant. From Eq. (4.4) we derive

$$
\begin{aligned}
& \mathbf{C}_{\mathbf{X}}(0)=\mathbf{A C}_{\mathbf{s}}(0) \mathbf{A}^{T}+\sigma^{2} \mathbf{I}, \\
& \mathbf{C}_{\mathbf{X}}(\tau)=\mathbf{A C}_{\mathbf{s}}(\tau) \mathbf{A}^{T}, \quad \tau \neq 0,
\end{aligned}
$$

where $\mathbf{C}_{\mathbf{X}}(\tau) \equiv\left\langle\mathbf{X}(t) \mathbf{X}(t+\tau)^{T}\right\rangle$ is the time-lagged covariance matrix of sample vector $\mathbf{X}$ with time-lag constant $\tau$ and we have assumed the random noises have identical distribution with standard deviation $\sigma$. Since the covariance matrix of the source signals $\mathbf{C}_{\mathbf{s}}(\tau)$ is diagonal, the mixing matrix $\mathbf{A}$ is the diagonalizer of $\mathbf{C}_{\mathbf{X}}(\tau)$.

In principle, source signal separation can be achieved by diagonalizing $\mathbf{C}_{\mathbf{X}}(\tau)$ with any time-lag constant $\tau$ if the matrix is non-degenerate. However, it is more reliable and more robust to use several time-lag covariance matrices instead of one. The mixing matrix $\mathbf{A}$ is found by jointly diagonalizing the covariance matrices with selected time-lag constants. The algorithm we use is the second order blind identification (SOBI) method [26], which is equivalent to the AMUSE algorithm with multiple time-lag constants [21]. The algorithm is listed below. 
First, compute the $m \times m$ sample covariance matrix $\mathbf{C}_{\mathbf{X}}(0) \equiv\left\langle\mathbf{X}(t) \mathbf{X}(t)^{T}\right\rangle$. Perform eigenvalue decomposition on $\mathbf{C}_{\mathbf{X}}(0)$ to obtain

$$
\mathbf{C}_{\mathbf{X}}(0)=\left(\mathbf{U}_{1}, \mathbf{U}_{2}\right)\left(\begin{array}{cc}
\boldsymbol{\Lambda}_{1} & 0 \\
0 & \boldsymbol{\Lambda}_{2}
\end{array}\right)\left(\begin{array}{c}
\mathbf{U}_{1}^{T} \\
\mathbf{U}_{2}^{T}
\end{array}\right),
$$

where $\boldsymbol{\Lambda}_{1}, \boldsymbol{\Lambda}_{2}$ are diagonal matrices with $\min \left(\operatorname{diag}\left[\boldsymbol{\Lambda}_{1}\right]\right) \geq \lambda_{c}>\max \left(\operatorname{diag}\left[\boldsymbol{\Lambda}_{2}\right]\right) \geq 0$, $\lambda_{c}$ is a cut-off threshold set to remove the singularity of the data matrix, and $\boldsymbol{\Lambda}_{1}$ is $n \times n$ diagonal matrix with eigenvalues $\lambda_{1} \geq \lambda_{2} \geq \cdots \geq \lambda_{n} \geq \lambda_{c}$. Using the matrix

$$
\mathbf{V} \equiv \boldsymbol{\Lambda}_{1}^{-1 / 2} \mathbf{U}_{1}^{T},
$$

we construct an $n$-component vector as $\xi=\mathbf{V X}$. The vector $\xi$ is called white because $\left\langle\xi \xi^{T}\right\rangle=\mathbf{I}$, where $\mathbf{I}$ is the $n \times n$ identity matrix. This step reduces the dimension of the data space, reduces the noise in the original data, and de-correlates and normalizes the data to facilitate the next step.

For a selected set of time-lag constants $\left\{\tau_{k}\right\}(k=1,2, \ldots, K)$, compute the timelagged covariance matrices $\left\{\mathbf{C}_{\xi}\left(\tau_{k}\right)=\left\langle\xi(t) \xi\left(t+\tau_{k}\right)^{T}\right\rangle\right\}$, form symmetric matrices $\overline{\mathbf{C}_{\xi}}\left(\tau_{k}\right)=\left(\mathbf{C}_{\xi}\left(\tau_{k}\right)+\mathbf{C}_{\xi}\left(\tau_{k}\right)^{T}\right) / 2$, and find a unitary matrix $\mathbf{W}$ that diagonalizes all matrices $\overline{\mathbf{C}_{\xi}}\left(\tau_{k}\right)$ of this set, i.e. $\overline{\mathbf{C}_{\xi}}\left(\tau_{k}\right)=\mathbf{W D}_{\mathbf{k}} \mathbf{W}^{T}$, where $\mathbf{D}_{\mathbf{k}}$ is diagonal. In practice, joint diagonalization can be achieved only approximately. Algorithms for approximate joint diagonalization can be found in Ref. [28].

Finally, the source signals and the mixing matrix are $\mathbf{s}=\mathbf{W}^{T} \mathbf{V X}$ and $\mathbf{A}=\mathbf{V}^{-1} \mathbf{W}$ respectively, where $\mathbf{V} \equiv \boldsymbol{\Lambda}_{1}^{-1 / 2} \mathbf{U}_{1}^{T}$ and $\mathbf{V}^{-1}=\mathbf{U}_{1} \boldsymbol{\Lambda}_{1}^{1 / 2}$.

For digitized sample data $X_{i}(t)$, constants $\tau_{k}$ are discrete integers. The expectation functional $\langle\cdots\rangle$ is replaced with sample average in practice. Improvement on the above algorithm have been studied by using robust whitening in Ref. [29, 30], or a combination of non-stationarity and time-correlation algorithm in Ref. [29]. In accelerator application, we find that our algorithm is sufficient to isolate all known independent signals. 
The application of ICA to beam diagnosis involves three phases: data acquisition and pre-processing, source signal separation and beam motion identification. To gain more information of the beam lattice, the beam needs to undergo coherent transverse motion while turn-by-turn data are taken. A pinger or rf resonant excitation kicker should be fired once or periodically to excite the beam.

The data sampled by BPMs around the ring are put into a data matrix as in Eq. (4.1). BPM gains may be applied to correct the BPM calibration error if necessary. The ICA algorithm is then applied to extract the mixing matrix $\mathbf{A}$ and source signals $\mathbf{S}$ from the data matrix $\mathbf{x}$. Each source signal $\mathbf{s}_{i}$ and its spatial distribution $\mathbf{A}_{\mathbf{i}}$, where $\mathbf{A}_{\mathbf{i}}$ is the $i$ 'th column of $\mathbf{A}$, is called a mode. The physical origin of a mode can be identified by its spatial and temporal vectors.

An oscillating signal (e.g. betatron oscillation) that has different phase at each BPM will appear as two modes with identical frequency spectra. Coherent betatron motion excited by the pinger should be damped by decoherence. An important signature of betatron modes is their tunes. Let $\mathbf{u}(t)$ be the betatron oscillation component of the transverse motion, then

$$
\mathbf{u}(t)=\mathbf{A}_{\mathbf{1}} s(t)+\mathbf{A}_{\mathbf{2}} c(t)
$$

where $s(t)$ and $c(t)$ are temporal patterns of the sine-like and cosine-like modes, respectively. The betatron amplitude function and phase advance can thus be derived

$$
\begin{aligned}
& \beta_{i}=a^{2}\left(A_{1, i}^{2}+A_{2, i}^{2}\right), \\
& \psi_{i}=\tan ^{-1}\left(\frac{A_{1, i}}{A_{2, i}}\right),
\end{aligned}
$$

where $a$ is a constant depending on initial conditions. The fractional part of the betatron tune can be obtained by the FFT of the temporal function. Advanced tune evaluation methods such as the interpolated FFT can be used to increase the accuracy [31]. 
The synchrotron mode can be recognized because its temporal pattern reflects the synchrotron oscillation of momentum deviation $\Delta p / p_{0}=\delta(t)$. The spatial pattern of the synchrotron mode is the dispersion function. Let $\mathbf{v}(t)$ be the synchrotron oscillation component in the transverse motion:

$$
\mathbf{v}(t)=\mathbf{A}_{s} s_{s}(t) .
$$

Note that there is only one mode because the synchrotron tune is much smaller than 1 , i.e., $\nu_{s} \ll 1$. The dispersion $D$ and the synchrotron coordinate $\delta(t)$ are related by

$$
\begin{gathered}
\mathbf{D}=b \mathbf{A}_{s}, \\
\delta(t)=s_{s}(t) / b
\end{gathered}
$$

with a constant $b$. The constant $a$ can be "determined" by the calibration of kicker strength and the "modeled" $\beta$ function at the kicker location through several kicker strengths. The constant $b$ can be calibrated through simultaneous phase measurement for the synchrotron motion.

In the presence of linear coupling, betatron motions of both transverse planes have two eigen-frequencies $\left(\nu_{+}\right.$and $\left.\nu_{-}\right)$. Consequently the betatron motion is decomposed into four modes, two with frequency $\nu_{+}$and the others with $\nu_{-}$. The betatron components of a horizontal and a vertical BPMs can be written in the form

$$
\begin{aligned}
& x(t)=A_{1} s_{-}(t)+A_{2} c_{-}(t)+a_{1} s_{+}(t)+a_{2} c_{+}(t), \\
& z(t)=b_{1} s_{-}(t)+b_{2} c_{-}(t)+B_{1} s_{+}(t)+B_{2} c_{+}(t),
\end{aligned}
$$

where $s(t)$ and $c(t)$ are the sine-like and cosine-like modes, respectively and subscripts "+/-" indicate the tunes. This decomposition corresponds to Edwards-Teng's parameterization theory of linear coupling [35], in which the betatron motion is expressed as a combination of two normal modes. The "+" modes and "-" modes 
correspond to the two normal modes of the theory. In Edwards-Teng theory, the general betatron motion takes the form

$$
\begin{aligned}
& x=\sqrt{W_{1} \beta_{1}} \cos \phi \cos \psi_{1}+\sqrt{W_{2}} \sin \phi\left[\left(d \sqrt{\beta_{2}}+\frac{b \alpha_{2}}{\sqrt{\beta_{2}}}\right) \cos \psi_{2}+\frac{b}{\sqrt{\beta_{2}}} \sin \psi_{2}\right], \\
& z=\sqrt{W_{1}} \sin \phi\left[\left(\frac{b \alpha_{1}}{\sqrt{\beta_{1}}}-a \sqrt{\beta_{1}}\right) \cos \psi_{1}+\frac{b}{\sqrt{\beta_{1}}} \sin \psi_{1}\right]+\sqrt{W_{2} \beta_{2}} \cos \phi \cos \psi_{2},
\end{aligned}
$$

where $\beta_{1,2}$ and $\psi_{1,2}$ denote the beta functions and phase advances of the normal modes, $W_{1,2}$ denotes the single-particle emittances which depend on initial conditions, parameters $a, b, d$ are related to coupling blocks of the local one-turn transfer matrix and the $\phi$ parameter is related to the coupling depth. The $\phi$ parameter depends on the location and varies across skew quadrupole components. By comparing Eq. (4.15),(4.16) to Eq. (4.17),(4.18), we see that

$$
\begin{aligned}
\sqrt{A_{1}^{2}+A_{2}^{2}} & =\sqrt{W_{1} \beta_{1}} \cos \phi \\
\sqrt{a_{1}^{2}+a_{2}^{2}} & =\sqrt{W_{2}} \sin \phi \sqrt{\left(d \sqrt{\beta_{2}}+\frac{b \alpha_{2}}{\beta_{2}}\right)^{2}+\frac{b^{2}}{\beta_{2}}} \\
\sqrt{b_{1}^{2}+b_{2}^{2}} & =\sqrt{W_{1}} \sin \phi \sqrt{\left(-a \sqrt{\beta_{1}}+\frac{b \alpha_{1}}{\beta_{1}}\right)^{2}+\frac{b^{2}}{\beta_{1}}} \\
\sqrt{B_{1}^{2}+B_{2}^{2}} & =\sqrt{W_{2} \beta_{2}} \cos \phi
\end{aligned}
$$

and

$$
\begin{aligned}
& \psi_{1}=\tan ^{-1}\left(\frac{A_{1}}{A_{2}}\right) \\
& \psi_{2}=\tan ^{-1}\left(\frac{B_{1}}{B_{2}}\right)
\end{aligned}
$$

So beta functions and phase advances of normal modes can be derived only from the proper components of the BPM readings, namely Eq. (4.19), (4.22) should be used for 
beta function measurements and Eq. (4.23), (4.24) for phase advance measurements. The variation of $\phi$ affects the measured beta function. However, such variation is usually very small. For example, when all the 48 skew quadrupoles of the Booster are powered with $2 \mathrm{~A}$ at $400 \mathrm{MeV}$ (corresponding to a focusing length of $f=180 \mathrm{~m}$ ) and the betatron tune separation is $\nu_{z}-\nu_{x}=0.038$, the rms variation of $\phi$ at the BPMs is only $2 \times 10^{-4}$. The correspondence of the "+/-" modes to the $\mathrm{H} / \mathrm{V}$ planes is based on the no-coupling case. In practice with experimental data, it is determined according to the normal behavior of the machine or by changing trim quadrupoles and watching how the tunes shift. If the horizontal plane is identified with the "-" mode, then the horizontal spatial vector of the "-" mode should be used to calculate beta function and phase advance of the horizontal plane (or the "-" mode). If the horizontal part of the "+" mode, i.e., the $\sqrt{a_{1}^{2}+a_{2}^{2}}$ term is also included in beta calculation, the result will be degraded. The vectors $a_{1,2}$ cannot be used to calculate the phase advance of the "+" mode because of the complication of the parameters $b$, $d, \alpha$ and $\beta$, which in general depend on the location in the ring. Similar arguments apply to the vertical plane and the "+" mode. The above observations have been verified in simulation studies using turn-by-turn tracking data as will be discussed later in this chapter.

Higher order resonances, if they appear, can be recognized by their characteristic frequencies. Other signals can also occur. For example, ripples of magnet power supply can modulate the beam transverse motion. BPMs may pick up an RF signal of a nearby equipment. Some BPMs may possess an artificial signal in their output due to circuit malfunction. These signals can be identified and studied. The unidentified remainder of the original sample signals may be considered as the random noise of the BPM system.

If transverse motion for both the horizontal $(\mathbf{x})$ and vertical $(\mathbf{z})$ planes can be 
recorded simultaneously, we should put them together in a matrix $\mathbf{y}=\left(\begin{array}{l}\mathbf{x} \\ \mathbf{z}\end{array}\right)$ for analysis. This will increase the precision of the mode separation because of the additional sample data.

\subsection{Simulation Studies}

The simulation studies are carried out with a simple model and with tracking data using the Booster lattice model.

\subsubsection{The simulation model with linear coupling}

We study an ideal lattice model with known analytic solution. Let the equations of motion be

$$
\begin{aligned}
& \frac{d^{2}}{d \theta^{2}} x+\nu_{x}^{2} x+C y=0 \\
& \frac{d^{2}}{d \theta^{2}} y+\nu_{y}^{2} y+C x=0
\end{aligned}
$$

where $\nu_{x}, \nu_{y}$ are betatron tunes, $C$ is the coupling constant and $\theta=2 \pi f_{0} t$ is the orbital angle with revolution frequency $f_{0}$. The model assumes continuous, uniform focusing with linear coupling. The solution of the coupled equations is

$$
\begin{aligned}
& x=A_{1} \cos \nu_{+} \theta+A_{2} \cos \nu_{-} \theta, \\
& y=B_{1} \cos \nu_{+} \theta+B_{2} \cos \nu_{-} \theta,
\end{aligned}
$$

where the eigen-tunes are given by

$$
\nu_{ \pm}^{2}=\frac{1}{2}\left(\nu_{x}^{2}+\nu_{y}^{2} \pm \sqrt{\left(\nu_{x}^{2}-\nu_{y}^{2}\right)^{2}+4 C^{2}}\right) .
$$


The coefficients are determined by initial conditions. For $x(0)=x_{0}, x^{\prime}(0)=0$, $y(0)=y_{0}$, and $y^{\prime}(0)=0$, they are given by

$$
\begin{array}{ll}
A_{1}=\frac{C y_{0}+\left(\nu_{x}^{2}-\nu_{-}^{2}\right) x_{0}}{\nu_{+}^{2}-\nu_{-}^{2}}, & A_{2}=-\frac{C y_{0}+\left(\nu_{x}^{2}-\nu_{+}^{2}\right) x_{0}}{\nu_{+}^{2}-\nu_{-}^{2}}, \\
B_{1}=\frac{C x_{0}+\left(\nu_{y}^{2}-\nu_{-}^{2}\right) y_{0}}{\nu_{+}^{2}-\nu_{-}^{2}}, & B_{2}=-\frac{C x_{0}+\left(\nu_{y}^{2}-\nu_{+}^{2}\right) y_{0}}{\nu_{+}^{2}-\nu_{-}^{2}} .
\end{array}
$$

We generate multiple BPM turn-by-turn data so that we can compare the results of PCA and ICA with the analytic solution. We place $M$ BPMs uniformly distributed around the ring, i.e. the phase advances at the $i$ 'th $(i=0,1, \cdots, M-1)$ BPM are $\psi_{+, i}=2 \pi \nu_{+} i / M$ and $\psi_{-, i}=2 \pi \nu_{-} i / M$. The readings this BPM will record are

$$
\begin{aligned}
& x_{i}(n)=A_{1} \cos \left(\nu_{+} n+\psi_{+, i}\right)+A_{2} \cos \left(\nu_{-} n+\psi_{-, i}\right), \\
& y_{i}(n)=B_{1} \cos \left(\nu_{+} n+\psi_{+, i}\right)+B_{2} \cos \left(\nu_{-} n+\psi_{-, i}\right),
\end{aligned}
$$

where $n$ is the turn number. We can introduce bad-BPM modes and add white Gaussian random noise to each individual BPM to simulate the effect of noise.

\section{The de-mixing}

With the linear coupling model, each BPM detects a mixture of the normal modes ('+' and ' - ' modes, referring to signals with frequency $\nu_{+}$and $\nu_{-}$, respectively). It is desirable to get the pure '+' modes and '-' modes. Since the betatron phase at each BPM is different, there are two ' + ' and two '-' modes. Using the spatial function of these modes, we can calculate the coefficients $A_{1}, A_{2}, B_{1}, B_{2}$ and the phase advances of the ' + ' and ' - ' modes. The coefficients allow us to derive beta functions and the coupling angle.

In our simulation studies we apply both PCA and ICA methods to compare their ability in mode separation. We find that the PCA method can separate the modes in most cases when the singular values (SV) of the '+' and the '-' modes are substantially different from each other. However, when the SVs approach each other, PCA 
always produces modes with mixing. The closer the SVs are, the stronger the mixing is. On the other hand, ICA doesn't show any dependence on the relative magnitudes of the SVs. Figure 4.1 shows the spatial functions and tunes of modes 1 and 3 derived from the ICA and PCA respectively. We note that the SVs of these modes are about equal, and the resulting PCA modes are mixed.

The different mode separation ability of the two methods can be illustrated with a simple example. The unique orthogonal basis that the PCA obtains is determined by the variances of the components, or the relative strength of the signals, which obviously does not concern the signals' other properties such as their power spectra or probability density functions. Let $s_{1}$ and $s_{2}$ be two normalized independent signals with $\left\langle s_{1} s_{1}\right\rangle=\left\langle s_{2} s_{2}\right\rangle=1,\left\langle s_{1} s_{2}\right\rangle=\left\langle s_{2} s_{1}\right\rangle=0$. Let the data matrix be given by $x_{1}=2 s_{1}+s_{2}$ and $x_{2}=s_{1}+2 s_{2}$. We can use a set of new basis vectors $z_{1}=$ $\frac{1}{\sqrt{2}}\left(s_{1}+s_{2}\right)$ and $z_{2}=\frac{1}{\sqrt{2}}\left(s_{2}-s_{1}\right)$ or any orthogonal transformation to form the basis vectors for the source signals. However we prefer the basis $s_{1}$ and $s_{2}$ because they are independent. The additional conditions that we use to characterize the independence of signals are $\left\langle s_{1}(t) s_{1}(t+\tau)\right\rangle=S_{1}(\tau),\left\langle s_{2}(t) s_{2}(t+\tau)\right\rangle=S_{2}(\tau)$, and $\left\langle s_{1}(t) s_{2}(t+\tau)\right\rangle=\left\langle s_{2}(t) s_{1}(t+\tau)\right\rangle=0$. The new basis will inevitably be found to be $s_{1}$ and $s_{2}$ when the auto-correlation condition is imposed.

In the linear coupling model, the sample of each BPM is a mixture of 4 source signals $s_{i}(t)(i=1,2,3,4)$ among which are two ' + ' modes that make a ' + ' mode subspace and two '-' modes that make a '-' mode subspace. The resulting basis $\mathbf{s}$ contains only 4 non-trivial components. The source signals are considered separated if two components of $\mathbf{s}$ are in the ' + ' subspace and the other two are in the '-' subspace. However if there is a component which crosses the ' + ' and '-' subspace, the modes are still mixed.

From the above discussion, we see that PCA does not guarantee separation of the source signals. The basis $\mathbf{z}$ vectors are an orthogonal transformation of the source 


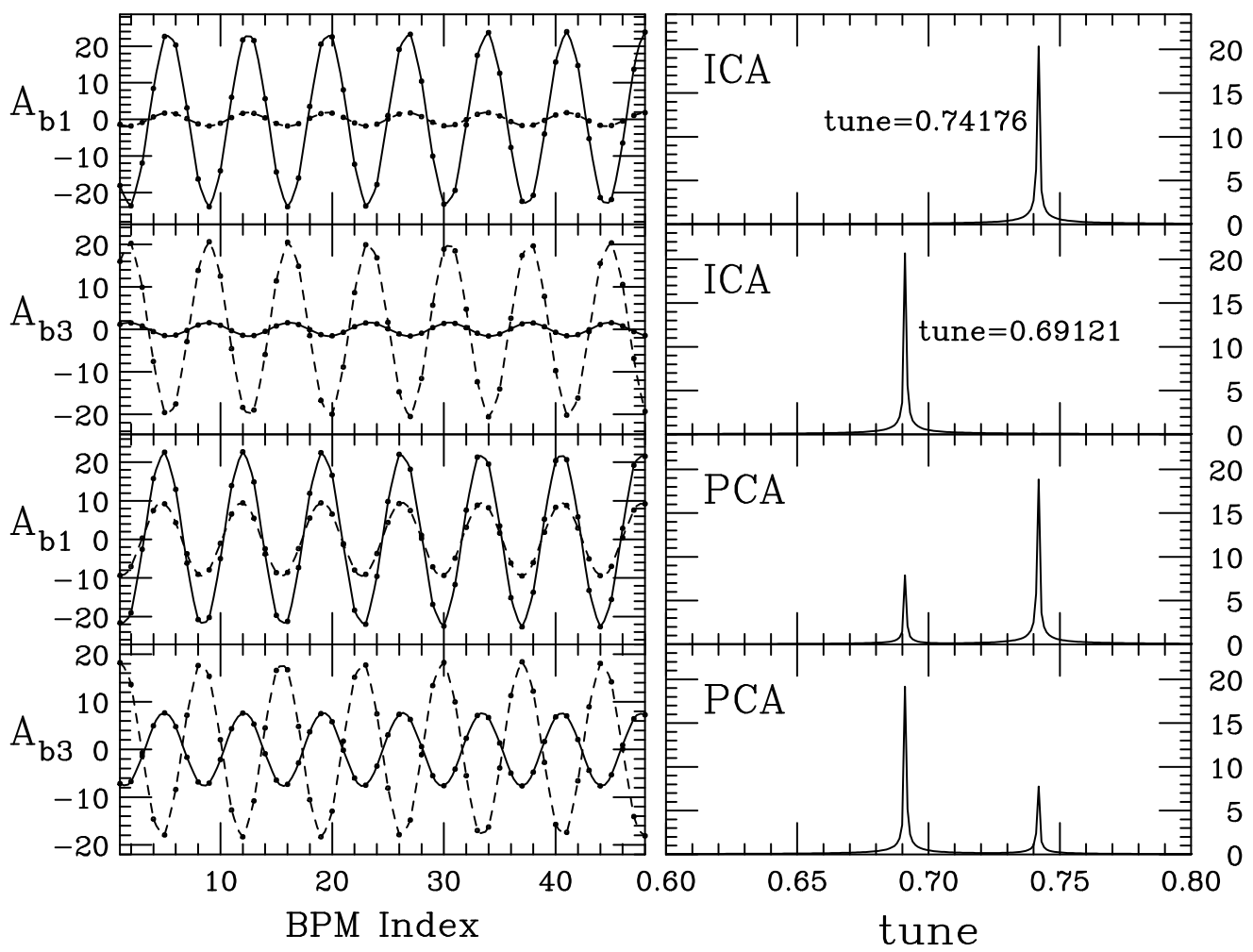

Figure 4.1: The mode separation ability of the ICA and PCA methods with linear coupling. The left column is spatial patterns of horizontal (solid) and vertical (dash) planes. The right column is the FFT spectra of the temporal patterns. Top and second rows: two ICA modes; third and bottom rows: two PCA modes. Model parameters are $\nu_{x}=6.74149, \nu_{y}=6.69149$, $C=0.05, x_{0}=y_{0}=1.0$. The SVs of the modes 1 and 3 are 122.0 and 98.8 respectively. 
signal basis vectors $\mathbf{s}$ depending on the strengths of the source signals in the sample data. The result depends on the distribution of the components of $\mathbf{x}$ in the space spanned by s. On the other hand, ICA makes use of the fact that the power spectra of source signals are distinct and the auto-correlation covariance matrices are diagonal to find the source signals.

\section{Effects of bad BPMs}

To further illustrate the behavior of PCA and ICA, we introduce a narrowband badBPM harmonic oscillation at a frequency far away from the betatron frequencies. This signal is added only to one BPM, i.e. the spatial vector of this mode is localized at a "bad" BPM. By changing the amplitude of this noise signal we can change the $\mathrm{SV}$ of this mode. It is observed that as the SV of this bad BPM mode is near that of the '-' modes or ' + ' modes, mode-mixing occurs. However the ICA mode is immune to such mode-mixing. The top two rows of Fig. 4.2 show that the betatron and the narrowband noise modes are mixed in PCA, where the SVs are 9.4 and 7.5 for the betatron and noise modes respectively. The bottom two rows of Fig. 4.2 show clearly that the ICA analysis is immune to mode mixing.

Another type of bad-BPM signal is white Gaussian noise. Applying noise to a single bad BPM and adjusting the noise level so that its SV is about the same as that of the betatron mode, we find that the PCA modes are mixed again. The results for both PCA and ICA are shown in Fig. 4.3, where the mode mixing occurs for PCA if the SVs of these two modes are close to each other. In the PCA, the betatron mode has leaked into the bad BPM mode as shown in the second row of Fig. 4.3. The ICA can easily isolate these two modes as shown in the row 3 and 4 of Fig. 4.3.

Since the PCA depends on the strengths of the source signals, it is sensitive to bad BPMs which are often noisy and strong. Thus it is absolutely necessary to exclude 
the bad BPMs before applying the PCA method. On the other hand, the ICA is more robust to bad-BPMs. This is an advantage, especially for on-line applications.

\section{The Effects of low level noises}

In reality BPM readings always contains random noise which affects the results of data analysis. We insert white Gaussian noise into the simulation data matrix. The rms errors of the resulting beta functions and phase advances of both the ICA and PCA methods are estimated as shown in Figure 4.4. The result agrees with the analytic error estimation of PCA in Eq. (17) of Ref. [17], which reads

$$
\sigma_{\psi} \approx \frac{1}{\sqrt{M}} \frac{\sigma_{r}}{\sigma_{s}}, \quad \sigma_{\Delta \beta / \beta} \approx 2 \sigma_{\psi},
$$

where $M$ is the number of BPMs, $\sigma_{r}$ is the BPM resolution, $\sigma_{s}=A / \sqrt{2}$ is the rms strength of the betatron signal and $A$ is the oscillation amplitude. It turns out that PCA and ICA have equal performances with respect to random noise. This is reasonable because ICA takes PCA as its first step (whitening) and white noise play little role in diagonalization of the unequal-time covariance matrices.

\section{The Effects of number of turns}

Both PCA and ICA are subject to deficiencies due to the finite sampling points. Both methods assume diagonal covariance matrix of the source signals, which is true only asymptotically, i.e. when the number of sampling points goes to infinity. In reality, the results of both PCA and ICA are affected by the number of sampling points. The off-diagonal elements of the covariance matrix of two harmonic signals can be estimated by

$$
\begin{aligned}
\left\langle s_{1}(t) s_{3}(t)\right\rangle & =\frac{1}{N_{t}} \sum_{n=0}^{N_{t}-1} \sin \left(2 \pi \nu_{+} n\right) \sin \left(2 \pi \nu_{-} n\right) \\
& \approx \frac{1}{2 N_{t}} \frac{\sin \pi \Delta \nu N_{t}}{\sin \pi \Delta \nu} \cos \pi \Delta \nu\left(N_{t}-1\right)
\end{aligned}
$$




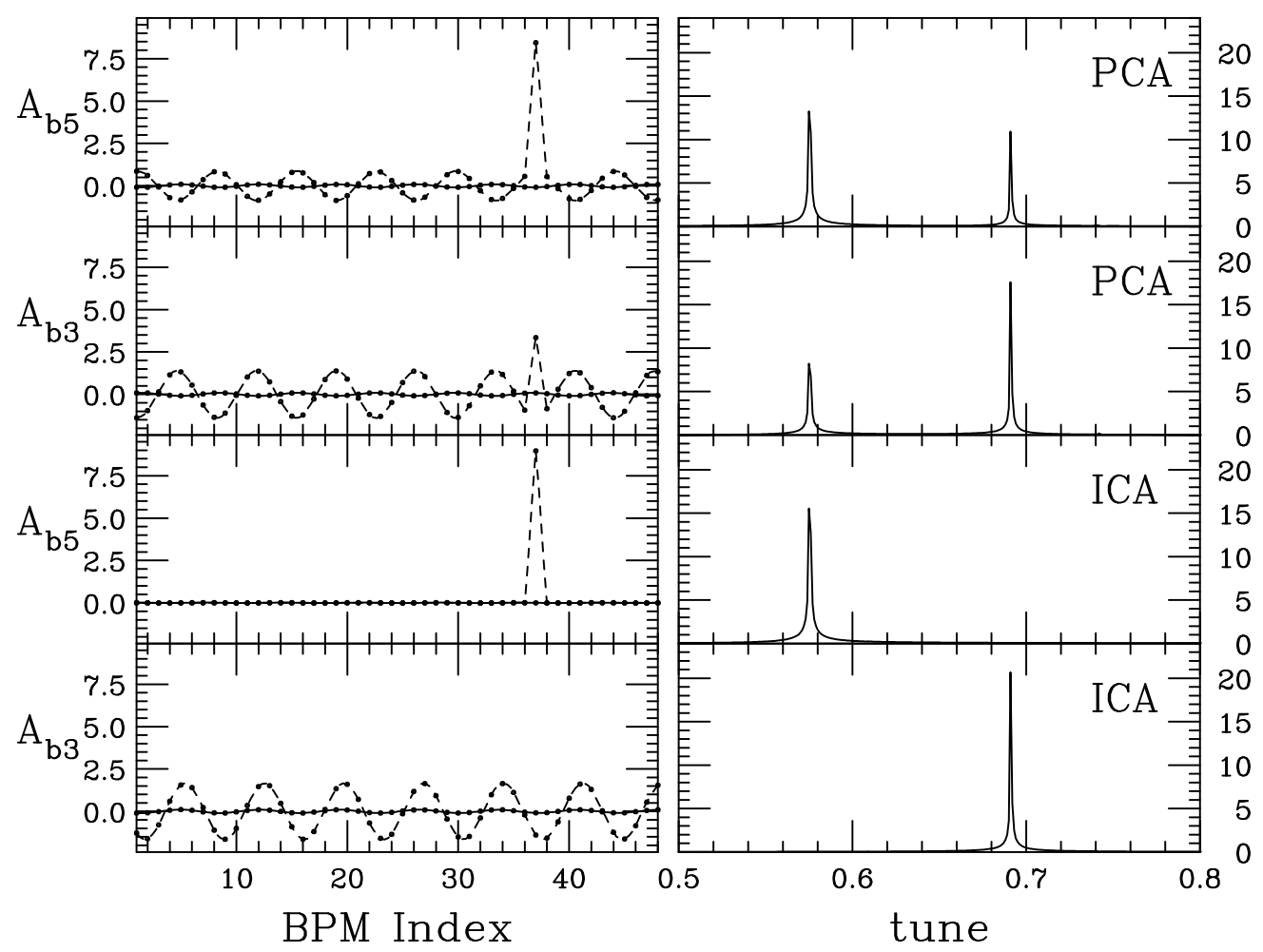

Figure 4.2: The PCA and ICA modes of the LC model with localized bad BPM signal. Model parameters: $\nu_{x}=$ $6.74149, \nu_{y}=6.69149, C=0.05, x_{0}=1.0, y_{0}=0.0$. A harmonic oscillation signal is added to BPM V37 with tune $\nu=0.57545$ and amplitude $D=0.4$. The top and the second rows: PCA modes with $\mathrm{SV}=9.4$ and $\mathrm{SV}=7.5$, respectively. These two modes are mixed. The third and the fourth rows: ICA modes of the same data. The localized 'bad-BPM' mode is completely separated. 


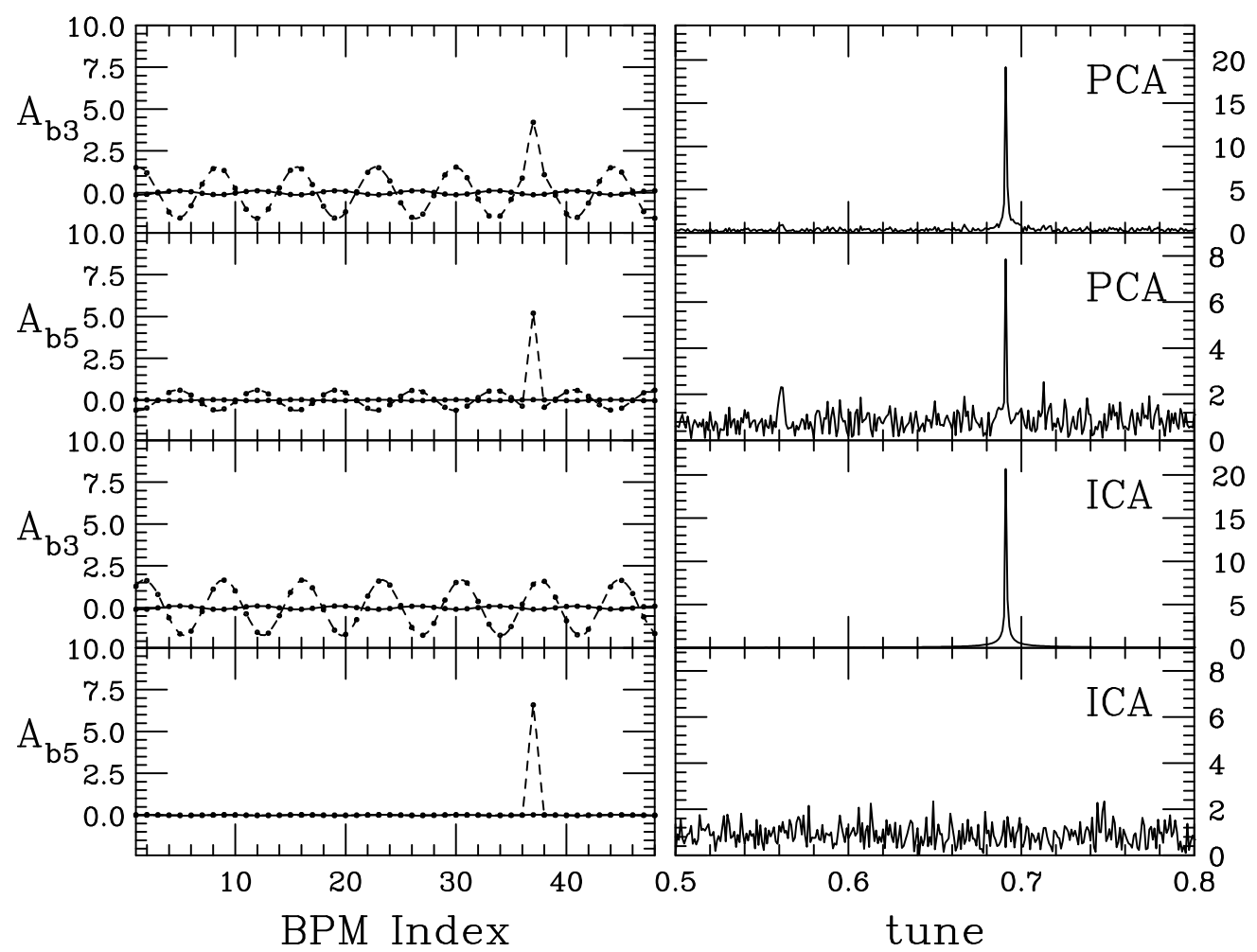

Figure 4.3: The PCA and ICA modes of the LC model with localized bad BPM with white Gaussian noise. Model parameters: $\nu_{x}=6.74149, \nu_{y}=6.69149, C=0.05$, $x_{0}=1.0, y_{0}=0.0$. The signal added to BPM V37 is white Gaussian noise. The top and the second rows are $\mathrm{PCA}$ modes with $\mathrm{SV}=8.4$ and $\mathrm{SV}=6.1$, respectively. The two PCA modes are mixed. The third and the fourth rows are ICA modes with the same data. The localized 'bad-BPM' mode is completely separated. 


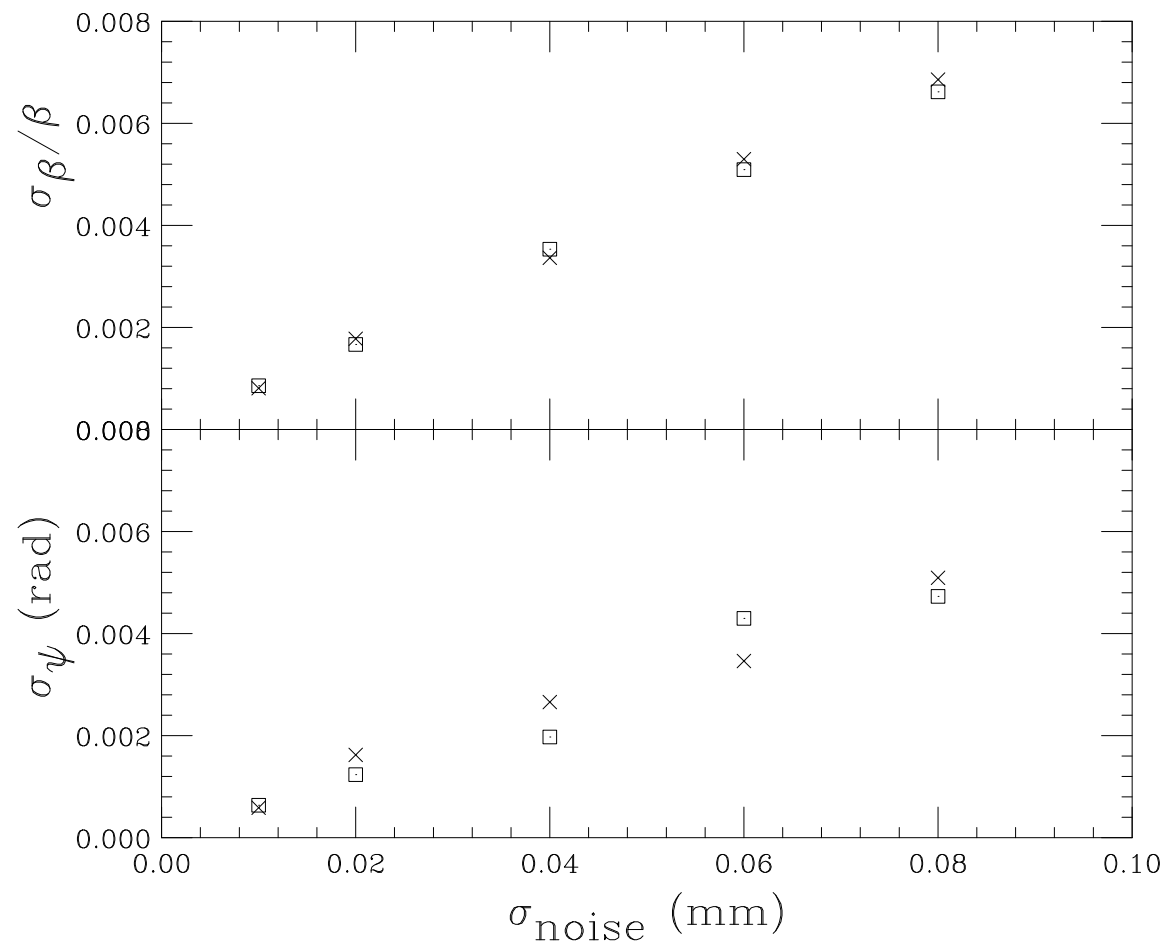

Figure 4.4: Estimation of errors of ICA (cross) and PCA (square) methods with various random noise levels in the LC model. The model parameters are the same as Figure 4.2. Data of 1000-turn are used to calculate $\sigma_{\beta} / \beta$ (top plot) and $\sigma_{\psi}$ (bottom plot). The estimation at each noise level $\sigma_{\text {noise }}$ (x-axis) is made by repeating the measurement of $\beta$ and $\psi 10$ times with white Gaussian random noises added to each BPM. 
where $s_{1}(t)$ and $s_{3}(t)$ are the two signals with tune $\nu_{+}$and $\nu_{-}$respectively, $\Delta \nu=$ $\nu_{+}-\nu_{-}$, and $N_{t}$ is the number of sampling points. The effects of $N_{t}$ to PCA and ICA can be simulated. Figure 4.5 shows the dependence of $\mathbf{C}_{\mathbf{s}}(1,3)=\left\langle s_{1}(t) s_{3}(t)\right\rangle$ on the sampling turns $N_{t}$ and the resulting error in $\sigma_{\beta} / \beta$ with respect to $N_{t}$. The ICA method is less affected by $N_{t}$ because its results are based on diagonalization of several auto-correlated matrices instead of only one.

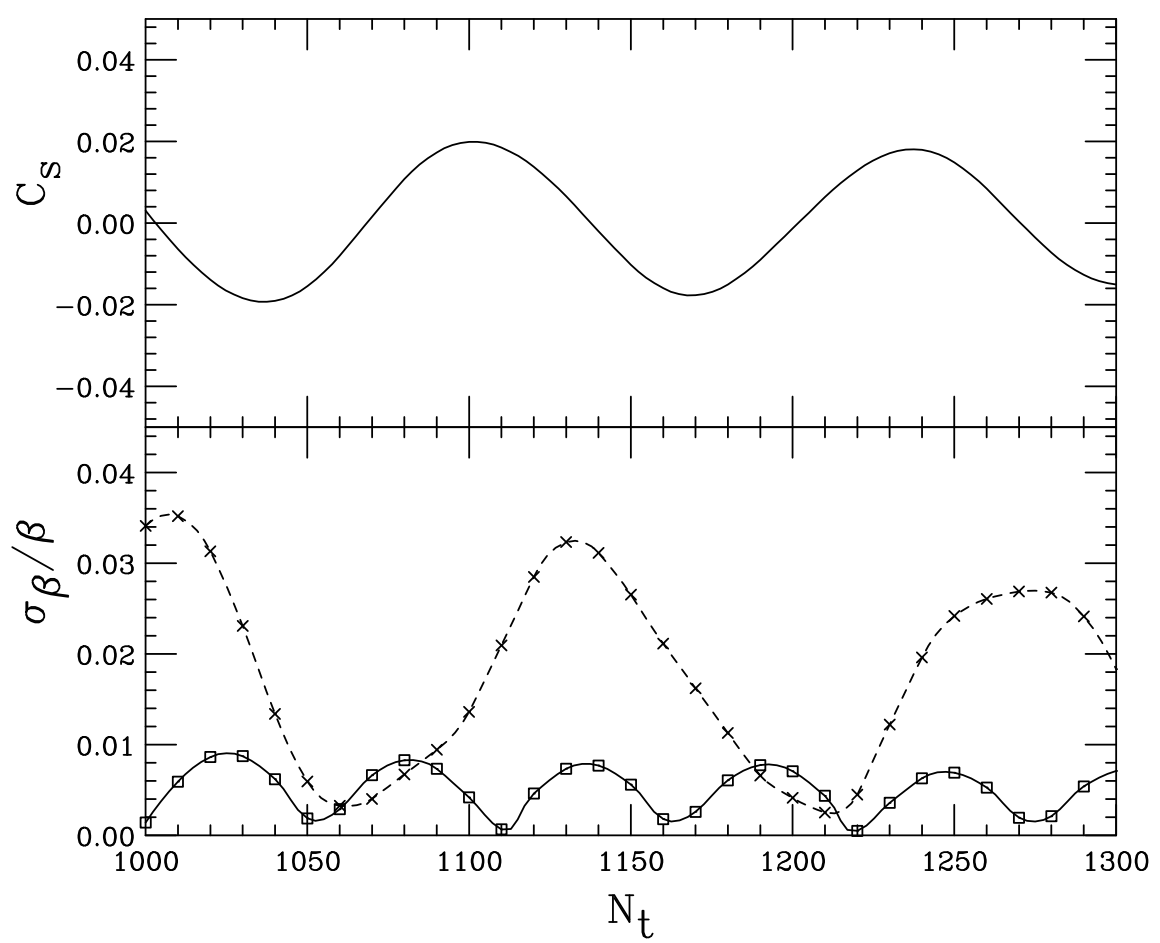

Figure 4.5: Effect of number of the sampled turns $\left(N_{t}\right)$ in the LC model with $\nu_{+}=6.7447$ and $\nu_{-}=6.7372$. Top: the off-diagonal element $C_{s}(1,3)$ of source signals. Bottom: $\sigma_{\beta} / \beta$ vs. $N_{t}$ for ICA (solid) and PCA (dash). 


\subsubsection{Application to tracking data}

To explore the capability of ICA in actual data analysis, the ICA technique is employed to process BPM data produced by tracking programs such as MAD. We simulated betatron motions with linear coupling and verified the correspondence of the ICA betatron modes and the normal modes of Edwards-Teng's theory. We show that the beta functions and phase advances should be derived from spatial patterns of proper normal modes for both transverse planes. The ICA method is also used to isolate the resonance tune lines and their spatial distribution.

\section{Linear coupling}

The tracking data is obtained with the linear Booster model using the TRACK command of the MAD program. The tracking particle starts from section S1 with horizontal position $x_{0}=5 \mathrm{~mm}$, vertical position $z_{0}=5 \mathrm{~mm}$ and the slopes $x_{0}^{\prime}, z_{0}^{\prime}$ are both zeros. Its position at all 48 BPMs are recorded for 1024 turns. The results are supplied to the ICA analysis to obtain four components as described in Eq. (4.15), (4.16). The linear coupling is introduced by setting the skew quadrupoles of the 48 corrector packages to $1 \mathrm{~A}$. The betatron tunes are changed to $\nu_{z}=6.78186$ and $\nu_{x}=6.74463$ without linear coupling by changing the trim quadrupoles. The betatron tunes of the coupled motion are $\nu_{-}=6.78252$ and $\nu_{+}=6.74397$. The ICA obtains the spatial vectors of both the ' + ' and '-' modes. From the discussions that follows Eq. (4.18), we see that we should use the horizontal spatial vectors of the '-' modes to calculate the horizontal beta function and phase advance and the vertical spatial vectors of the '+' modes for vertical beta function and phase advance.

The 4D one-turn map at each BPM is also obtained with the MAD lattice model. From the maps one can derive the parameters $\phi, a, b$ and $d$ and the Courant-Snyder parameters $\alpha_{1,2}, \beta_{1,2}$ according to Ref. [35]. Then we can derive the single particle 
emittance $W_{1,2}$ with the spatial patterns of the normal modes and these parameters using Eq. (4.19) to Eq. (4.22). The result is shown in Fig. 4.6. The fact that $W_{1,2}$ derived from amplitudes of normal modes at all locations are identical proves Eq. (4.19) and Eq. (4.22). The phase advances are also derived from the spatial patterns with Eq. (4.23), (4.24) and compared to model calculation. The difference is negligible, with rms difference below $5 \times 10^{-4} \mathrm{rad}$, compared to average phase advance of $0.85 \mathrm{rad}$ from BPM to BPM.

\section{Nonlinear resonances}

Nonlinear magnetic fields (sextupole or higher order components) can excite resonances of the beam motion that correspond to tune lines which are linear combinations of the two betatron tunes $\nu_{x}$ and $\nu_{z}$. The tunes $m \nu_{x}+n \nu_{z}+p$, where $m, n$ and $p$ are integers, can appear in the spectrum of the turn-by-turn beam motion. When the nonlinear field is not strong enough to cause chaotic behavior, these tunes are discrete on the spectrum and can be separated with the ICA method. We have applied this method to the tracking data obtained with the Booster model. Including excitation of sextupoles and skew quadrupoles, we can easily separate modes associated with resonances such as $\nu_{x} \pm \nu_{z}, 2 \nu_{x}, 2 \nu_{z}, 2 \nu_{x} \pm \nu_{z}$ and $2 \nu_{z}-\nu_{x}$. Fig. 4.7 shows an example of a third order resonance mode corresponding to $-\nu_{x}+2 \nu_{z}-1=0$. The amplitude of nonlinear modes at each location is an indication of the local nonlinear one-turn map. Although the tracking data can be used to construct the Poincaré surface of section [34], the physical meaning of the spatial vector has not been fully understood. Since the signals of higher order resonance in the nominal operational condition of the Fermilab Booster are usually buried under the noise floor, it is not likely that we can study the importance of the nonlinear resonance with experimental turn-by-turn data. The ICA method may provide an alternative approach to the Frequency Map 


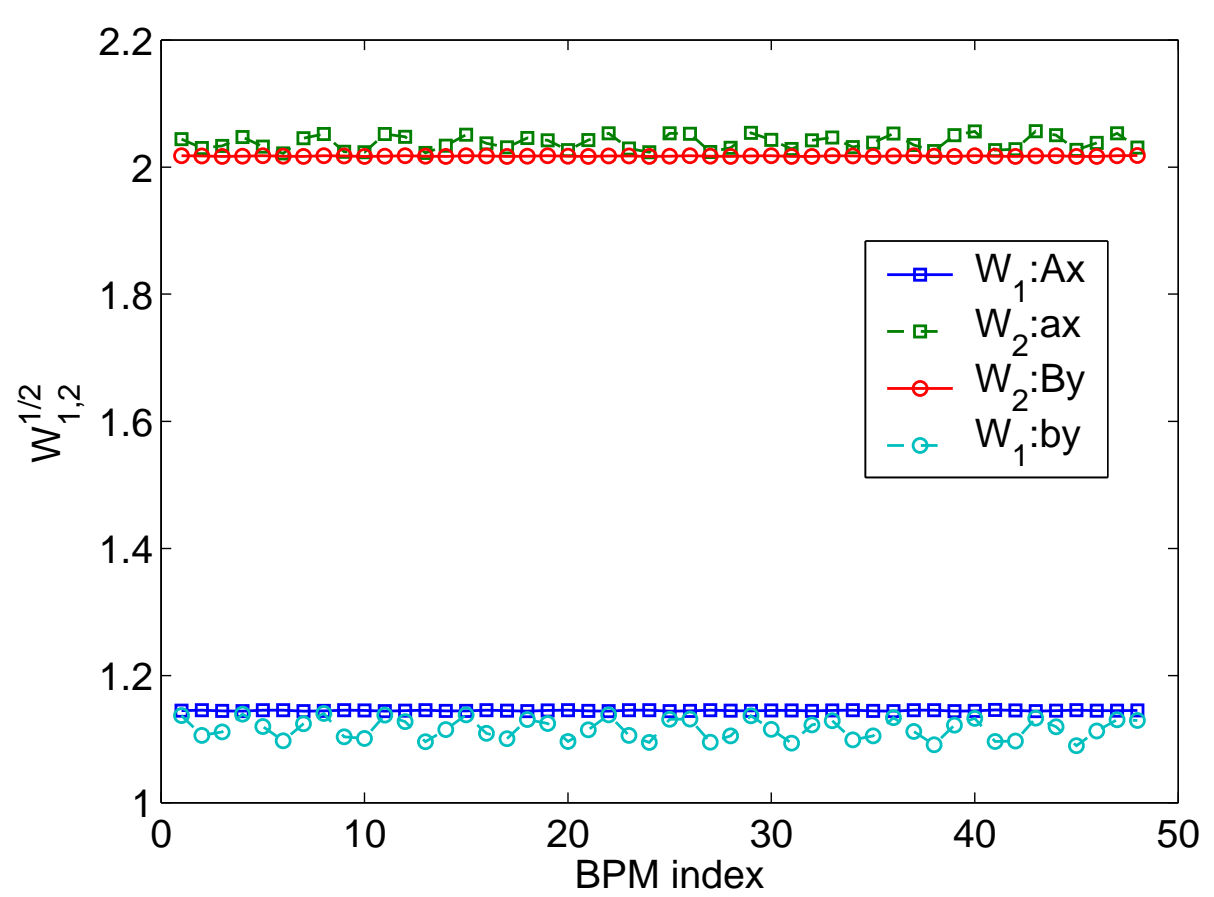

Figure 4.6: (Color) Square root of the single-particle emittance $\sqrt{W_{1,2}}$ derived from Eq. (4.19) to Eq. (4.22) using the spatial vectors of normal modes and the Edwards-Teng parameters from local 4D one-turn maps. "W $\mathrm{W}_{1}: \mathrm{Ax} "$ (blue square solid) is $\sqrt{W_{1}}$ derived from horizontal minus mode; "W $:$ ax" (green square dash) is $\sqrt{W_{2}}$ derived from horizontal plus mode; "W $\mathrm{W}_{2}$ By" (red circle solid) is $\sqrt{W_{2}}$ derived from vertical plus mode; "W $\mathrm{W}_{1}$ :by" (cyan circle dash) is $\sqrt{W_{1}}$ derived from vertical minus mode. 
Analysis (FMA) as suggested in Ref. [36].

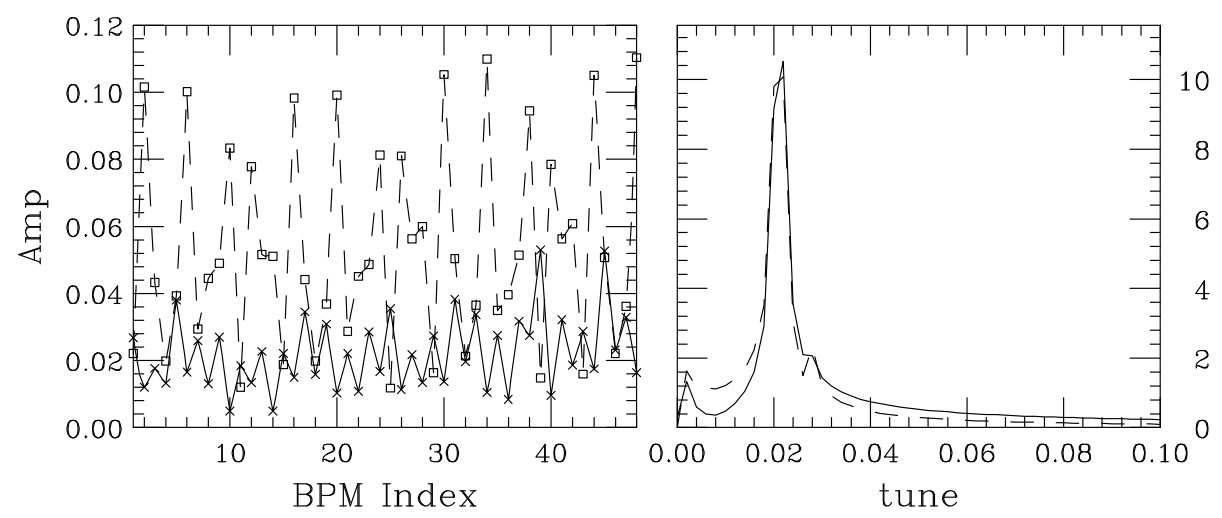

Figure 4.7: Third order resonance signals corresponding to $-\nu_{x}+$ $2 \nu_{z}-1=0$ in tracking data (500 turns) of the Booster. The tune of the signals is 0.02107, while $\nu_{x}=6.65753$, $\nu_{z}=6.83929$. The currents in sextupole families are $\mathrm{SEXTL}=20 \mathrm{~A}, \mathrm{SEXTS}=5 \mathrm{~A}$. Left plot: Amplitude of the resonance signal at horizontal (solid cross) and vertical (dash square) BPMs. Right plot: the FFT spectrum of the two resonance signals.

\subsection{Lattice Modeling with Measured Lattice Func- tions}

The ICA method provides a means to measure the linear optics functions such as beta functions, phase advances and dispersion. These functions can be used to correct the accelerator model (e.g., MAD) by tuning the model to minimize the difference between calculation and measurement. We use the Booster model to illustrate this 
new approach of lattice modeling below. The model parameters to be varied are the quadrupole gradients $K_{1}$ of the 96 main magnets.

We define the merit function

$$
\begin{aligned}
f(\mathbf{q}) & =\chi^{2}=\frac{1}{2} \sum_{i=1}^{240} r_{i}^{2} \\
r_{i} & =\frac{y_{i}(\mathbf{q})-y_{i}^{d}}{\sigma_{i}} \\
\mathbf{y} & =\left(w_{1} \beta_{x}, w_{2} \boldsymbol{\Delta} \psi_{x}, w_{3} \beta_{y}, w_{4} \boldsymbol{\Delta} \psi_{y}, w_{5} \mathbf{D}_{x}\right),
\end{aligned}
$$

where $\mathbf{q}$ is a $96 \times 1$ vector consisting of the body quads corrections (i.e., $\Delta K_{1}$ ), $\beta_{x}$, $\boldsymbol{\Delta} \psi_{x}, \beta_{y}, \boldsymbol{\Delta} \psi_{y}, \mathbf{D}_{x}$ are all row vectors with 48 components at 48 BPM locations, $\mathbf{y}(\mathbf{q})$ and $\mathbf{y}^{d}$ are both $1 \times 240$ vectors containing the model and measured linear optics functions, respectively, $\sigma_{i}$ is the corresponding error estimation of $y_{i}^{d}$ serving as weight in the definition. The additional weights $w_{i}, i=1,2, \cdots, 5$ are used to account for our confidence over the five categories of fitting data. We may set them to $w_{1}=w_{3}=w_{5}=1$ and $w_{2}=w_{4}=4$ to put more weights on the phase advances because they are independent of BPM calibration.

This non-linear least square problem can be solved iteratively by LevenbergMarquardt method $[12,13]$. In each iteration we compute the Jacobian matrix defined as

$$
J_{i j}=\frac{\Delta y_{j}}{\Delta q_{i}}
$$

and solve

$$
\left(\mathbf{J}^{T} \mathbf{J}+\lambda \mathbf{I}\right) \Delta \mathbf{q}=-\mathbf{J}^{T} \mathbf{r}_{0}
$$

to obtain a new set of fitting parameters, where $\mathbf{I}$ is the identity matrix and $\lambda$ is an adjustable non-negative parameter to control the behavior of the algorithm.

This fitting scheme has been applied to MAD simulation data. In one simulation, we put changes $\Delta K_{1}=0.0011 \mathrm{~m}^{-2}$ to six focusing magnets and $\Delta K_{1}=-0.00825 \mathrm{~m}^{-2}$ 
to six defocusing magnets to generate simulation data. The algorithm precisely converges to the expected solution. In more general cases, we find this method also suffers from the correlation between the neighboring magnets as the ORM fitting does. However, it is able to obtain an equivalent lattice model with the same lattice functions used in the fitting.

For the more common synchrotrons which use separate-function magnets, the correlation should be weaker because of the betatron phase advances between the quadrupoles are usually larger. This fitting scheme could be a useful method for lattice modeling. In particular, we believe the combination of the lattice function measurements with the orbit response matrix (ORM) could improve the robustness of fitting for those machines whose ORM fitting also tend to have non-unique solutions. 


\section{Chapter 5}

\section{Application of ICA to the Fermilab Booster}

All 48 BPMs of the Booster are able to digitize their readings turn by turn for the entire cycle. It is very suitable for the analysis of ICA. By applying this technique to experimental turn-by-turn BPM data of the Booster under different settings, we have improved our understanding of the Booster in several ways. We measured the linear lattice functions, including beta functions, betatron phase advances and dispersion function. The measurements were carried out before and after the re-positioning of the second dogleg magnet set (in section L03). For both cases, the measured lattice functions were compared to the model. We observed synchrotron motion in the Booster even though we didn't excite it intentionally. The amplitude of synchrotron motion in the Booster is usually weak. The momentum deviation shows variation in one turn and the distribution is correlated with the rf cavities. We measured the transverse impedance by the betatron tune shifts due to increased beam intensities. Chromaticity was also measured using the tune shift due to radial orbit offset.

Some data sets were taken in the DC mode, which means the beam is kept at 
injection energy by turning off most of the rf cavities. There are about 15,200 turns in a $\mathrm{DC}$ cycle. Other data sets were taken in regular ramping mode, or $\mathrm{AC}$ mode. The ramping cycle contains 20,000 turns. In both cases, the transverse motion is excited by a pinger which kicks the beam suddenly. The Booster pinger is a fast kicker magnet with a transmission line type structure. Its fill time is $20 \mathrm{~ns}$. In $8 \mathrm{GeV}, 1 \mathrm{kV}$ voltage applied to it produces $5.3 \mu \mathrm{r}$ bend [37]. The excitation is applied periodically, usually with an interval of $0.5 \mathrm{~ms}$ or 225 turns at injection. The voltage is $600 \mathrm{~V}$ at $400 \mathrm{MeV}$ and is ramped up to $3.8 \mathrm{kV}$ in $\mathrm{AC}$ mode to compensate the increase of beam rigidity. The pinger causes coherent betatron motion with maximum amplitude of about $0.5 \mathrm{~mm}$. Horizontal and vertical data are always taken and analyzed together. BPM gains obtained from the ORM study were used to correct the raw readings.

Due to unforeseen hardware problems, the raw readings of different BPMs are not synchronized. It is necessary to adjust the turn index until it is correct for every BPM. The burst position corresponding to the time when the pinger is fired is used to attain a coarse match and the fact that the betatron phase advance per period is about 100 degrees is used to determine the actual turn number precisely. The corrector settings, including trim quadrupoles, skew quadrupoles and sextupoles are recorded to construct a "precise" model for comparisons with the measurements.

The experimental results will be presented in separate sections in this chapter. The measurements of linear lattice functions are given in section 5.1. The transverse impedance measurements are presented in section 5.2. The chromaticity measurements are given in section 5.3. The observation of synchrotron motion in AC mode is shown in section 5.4. A brief summary in found in section 5.5. 


\subsection{Linear lattice function measurements}

\subsubsection{Data taken in DC mode}

The DC mode turn-by-turn data were taken in November 2003 after the dogleg magnets in L13 were re-positioned to increase their separation. The beam energy was kept at $400 \mathrm{MeV}$ for $33.3 \mathrm{~ms}$ or a total of 15200 turns. The Linac beam was injected to the Booster for four turns, corresponding to a total charge of 2.0E12 protons per pulse. All skew quadrupoles were turned off. The trim quadrupoles in short straight sections were turned off. Those in long straight sections were set to $0.2 \mathrm{~A}$ as it was necessary to keep the beam stable. The chromaticity sextupoles were set to 8 A for SEXTS and 11 A for SEXTL, respectively.

The pinger was fired horizontally every $0.5 \mathrm{~ms}$. Each pulse lasts $2.0 \mu \mathrm{s}$ or nearly one turn at $400 \mathrm{MeV}$. Because each burst of coherent betatron motion has 225 turns, the entire cycle is divided into small pieces such that each piece corresponds to one burst. The ICA method is applied to each piece. However, pieces of 1000 turns or more are used for analysis in some cases to study processes involving longer time scale.

Clean coherent betatron components were obtained with the ICA method. An example of extracted horizontal betatron modes are given in Fig. 5.1. The time-lag constants are chosen to be $\tau=[0,1,2,3]$. The power spectra of the two modes are shown in Fig. 5.2, which clearly indicate that their spectra are the same. Using Eqs. (4.10), (4.11), the betatron function and phase advance are derived from the spatial patterns. The measurements can be done with every burst of coherent betatron motion caused by the pinger. Using twenty bursts from two data sets taken in two cycles, we have estimated the error bars by the standard deviations of these measurements. The error bars of $\frac{\Delta \beta}{\beta}$ are $6 \%$ on average. The average error bar of phase advance $\Delta \phi$ 
between adjacent BPMs is $0.03 \mathrm{rad}$. The results are compared to model calculations in Fig. 5.3. The difference between the model and the measurement is $10 \%$ in terms of $\frac{\Delta \beta}{\beta}$ and $0.08 \mathrm{rad}$ in terms of $\Delta \phi$. Coherent vertical betatron motion is also excited through linear coupling. But the coupling strength is not very strong (see the lower plots of Fig. 5.1). The vertical betatron motion is also plagued by instability modes. It is not suitable for lattice function measurement.

Dispersion were also measured with DC data. There was an injection energy mismatch in the Booster. This energy error gradually disappeared because of the radial position feedback loop. The time evolution of the momentum deviation corresponds to a horizontal orbit drift. The ICA method can separate such a drift as an independent mode and its spatial patten is proportional to the dispersion function. The evolution of momentum deviation was derived from the temporal pattern. The initial momentum deviation was found to be $-0.4 \times 10^{-3}$, corresponding to an energy error of $\Delta E=-0.08 \mathrm{MeV}$. The results are shown in Fig. 5.4.

\subsubsection{Data taken in AC mode}

AC mode is the normal Booster operation mode. It is very convenient to take turnby-turn BPM data in AC mode. We took turn-by-turn data after the dogleg magnet set at section L13 was re-positioned (during the fall shutdown in 2003) to reduce the dogleg effect and more recently when the dogleg magnets at section L03 were also re-positioned (in fall 2004). The pinger worked horizontally before November 2004 and since then has been set to work vertically.

We first show data taken in April 2004 before the second dogleg was re-positioned. The pinger was set to kick the beam horizontally every $0.5 \mathrm{~ms}$. The beam intensity corresponded to four-turn injection. Because of the linear coupling, coherent betatron motion was also observed in the vertical plane in the first few thousand turns. The 

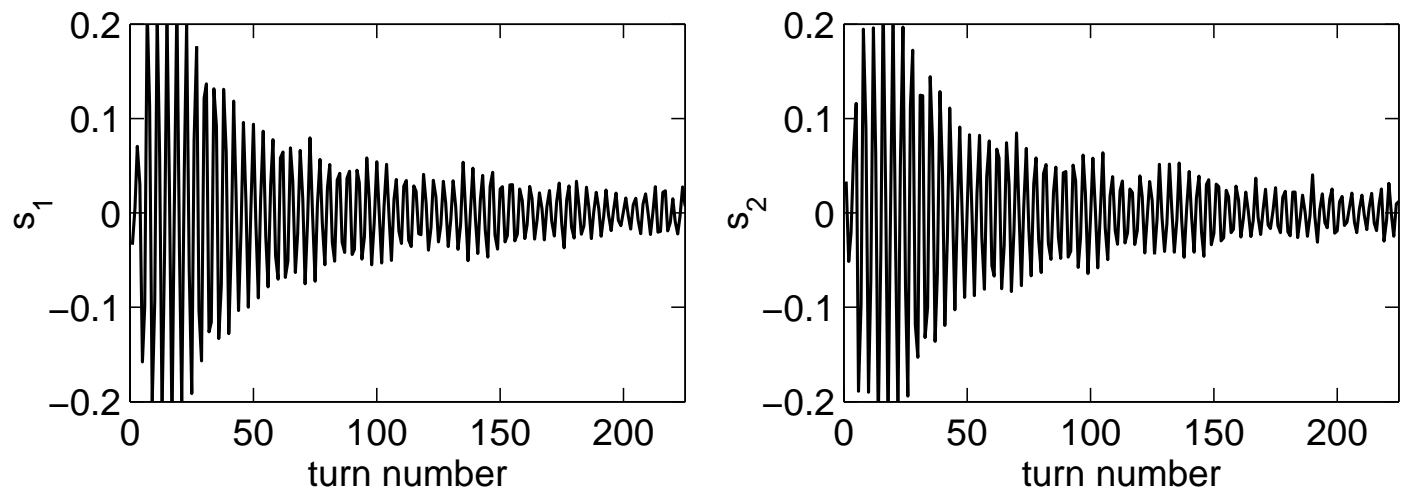

(a) temporal patterns
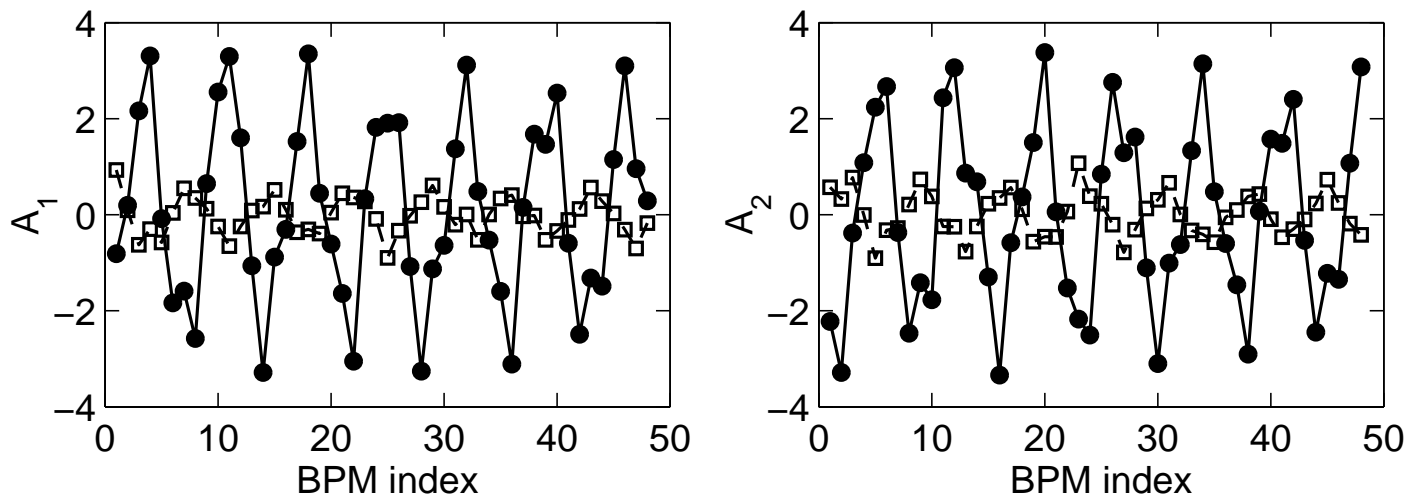

(b) spatial patterns

Figure 5.1: Examples of a pair of betatron modes with DC data at turn 1915:2140. (a) Temporal patterns. (b) The corresponding spatial patterns with both horizontal (solid circle) and vertical (hollow square) BPMs. 


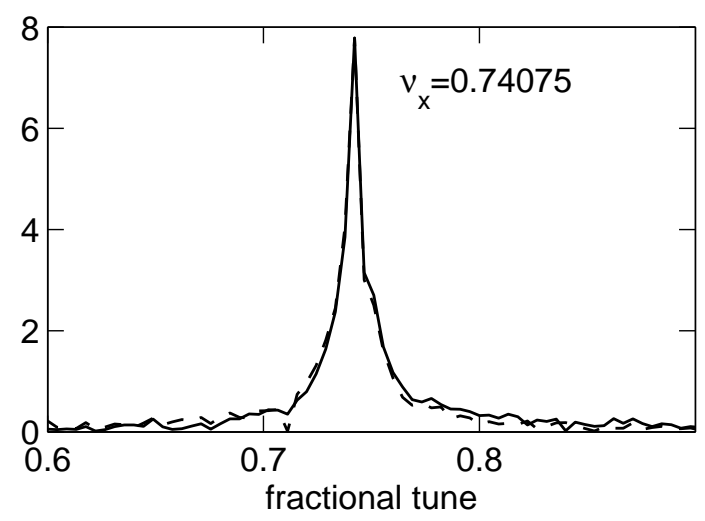

Figure 5.2: FFT spectra of the modes of DC data.

ICA method was used to separate the horizontal and vertical coherent motions, where their temporal and spatial functions of the betatron normal modes can be obtained. Fig. 5.5 shows the temporal patterns of the horizontal ("minus") and vertical ("plus") betatron modes for a burst from turn 2060 to turn $2320^{1}$. It is noticed that in this region the vertical decoherence occurs very slowly and it is not completely damped out before the next burst.

The corresponding spatial patterns of the betatron modes are shown in Fig. 5.6. Fig. 5.6 indicates strong linear coupling between betatron motions of the two transverse planes because it shows that the "plus" mode and the "minus" mode show up in both planes with nearly equal amplitude. The horizontal and vertical beta functions are measured and the results are shown in Fig. 5.7. Error bars are estimated with the standard deviations of results from four data sets. The average error bar of $\Delta \beta / \beta$ is $8 \%$ for horizontal and $17 \%$ for vertical data. The difference of model calculation and measurements in terms of average $\Delta \beta / \beta$ is $15 \%$ for both planes.

\footnotetext{
${ }^{1}$ Since usually the smaller betatron tune is the horizontal tune and the larger tune is vertical for the Booster, we refer the "minus" mode as horizontal mode and the "plus" mode as vertical mode.
} 

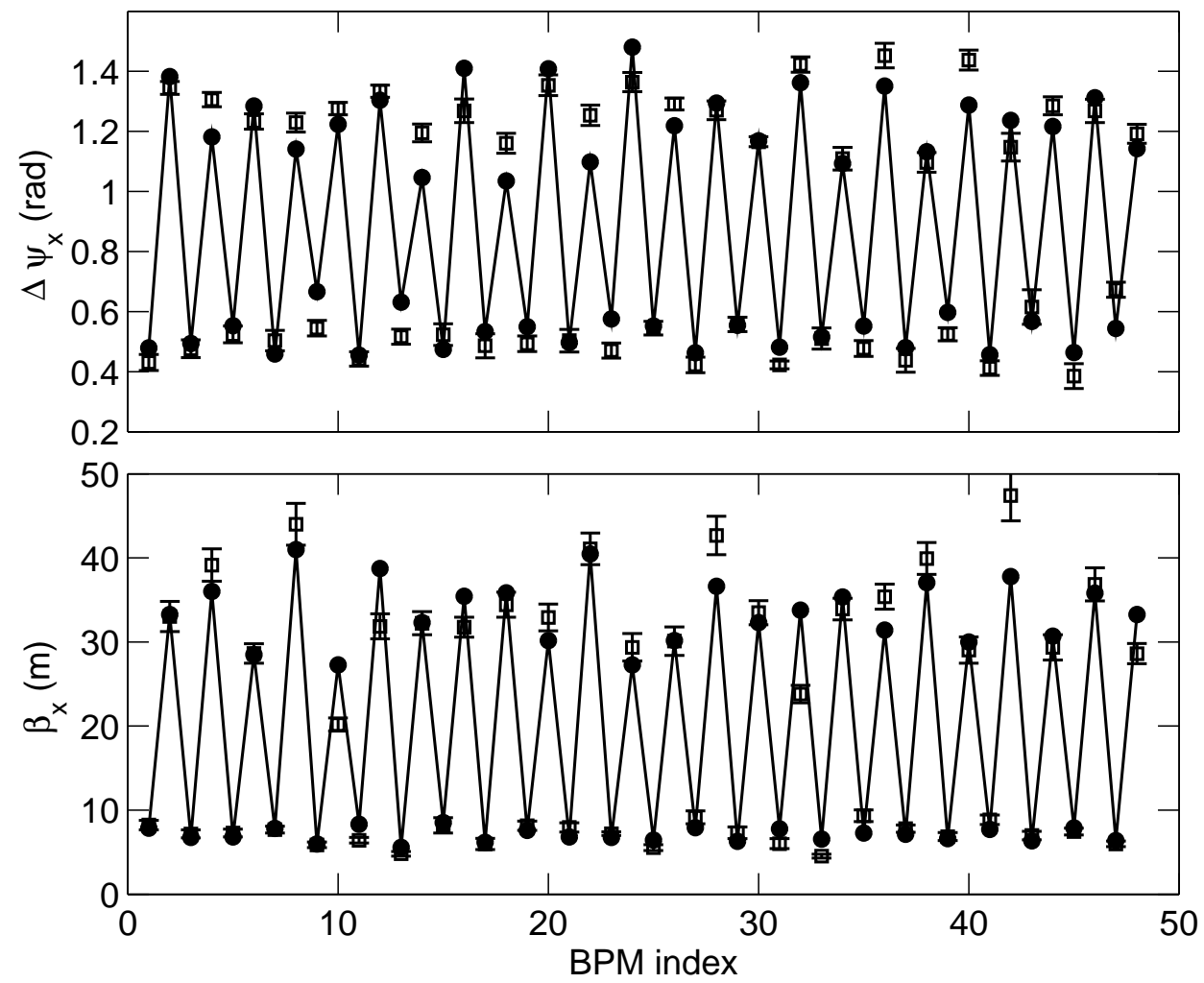

Figure 5.3: The measured (square) horizontal beta function $\beta_{x}$ (bottom) and betatron phase advance between adjacent BPMs (top) are compared to model calculation (solid circle). 

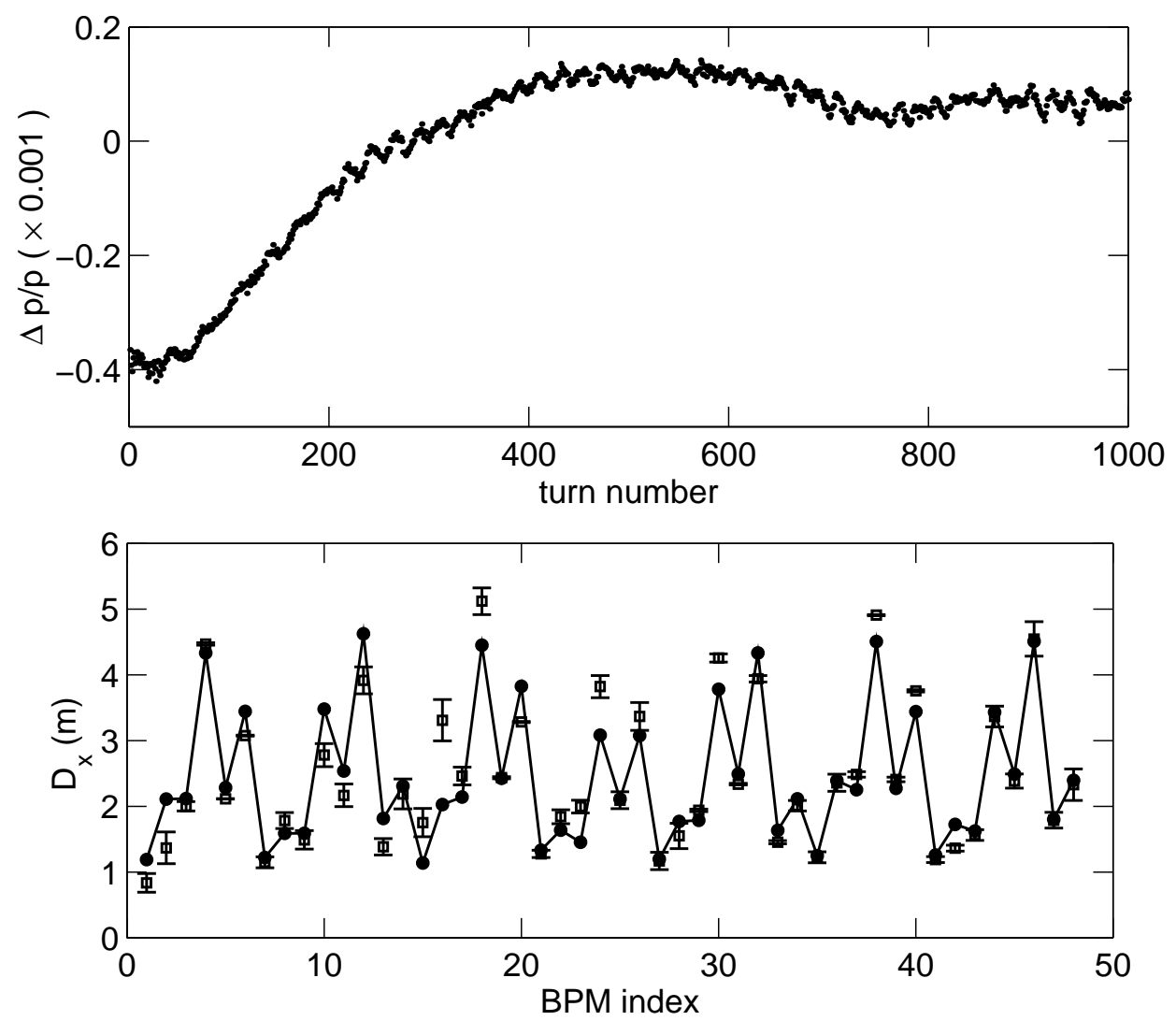

Figure 5.4: Top: the evolution of momentum deviation error after injection. Bottom: the dispersion function measured (square) with the spatial pattern is compared to model calculation (solid circle). 

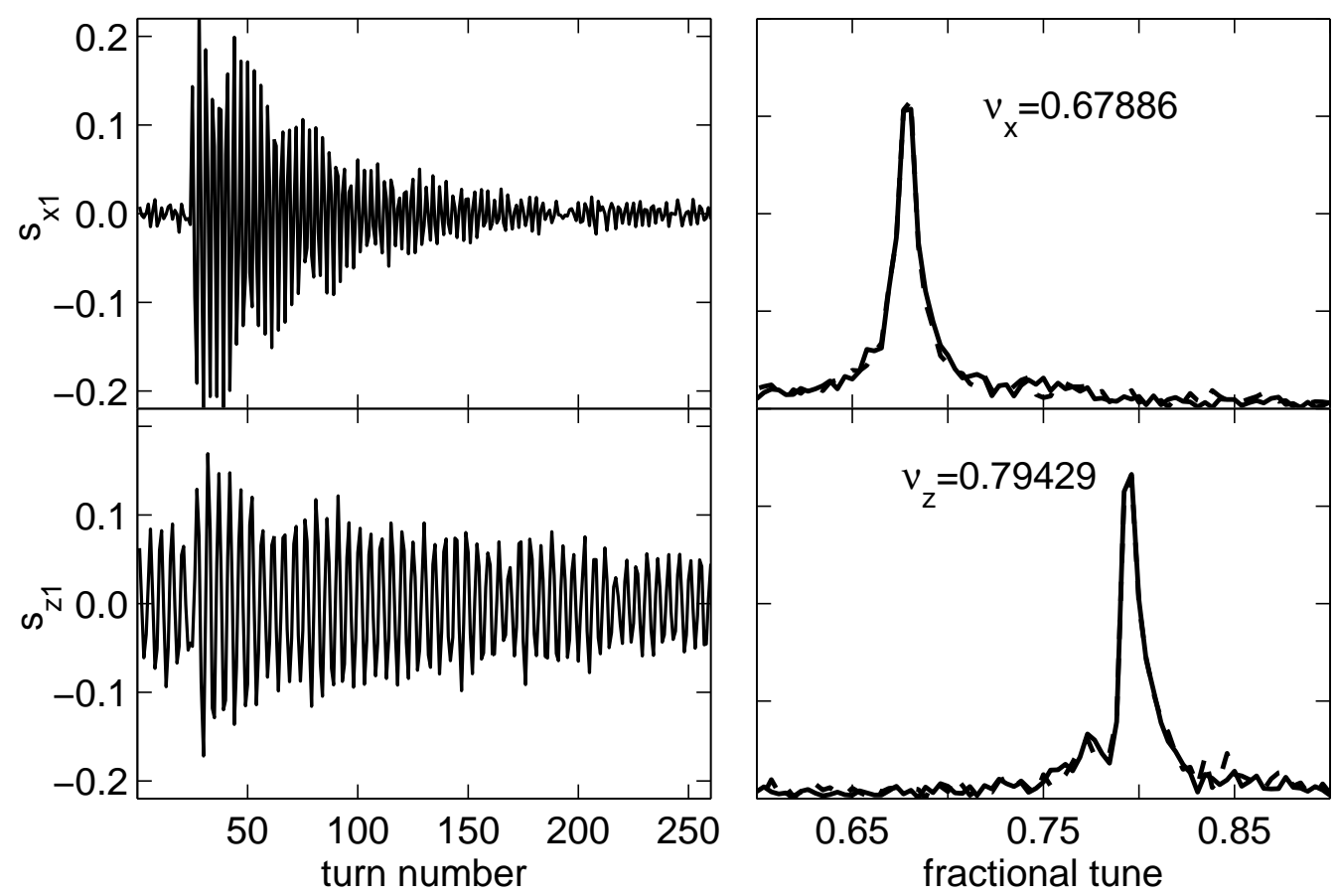

Figure 5.5: Temporal patterns of the betatron modes of AC data for the burst in turn 2060:2320. Top: the temporal pattern of a horizontal betatron mode (left) and the FFT spectra of the two (solid and dash) horizontal betatron modes (right). Bottom: the temporal pattern of a vertical betatron mode (left) and the FFT spectra of the two vertical betatron modes (right). The FFT spectra of the coupled modes are almost identical. 

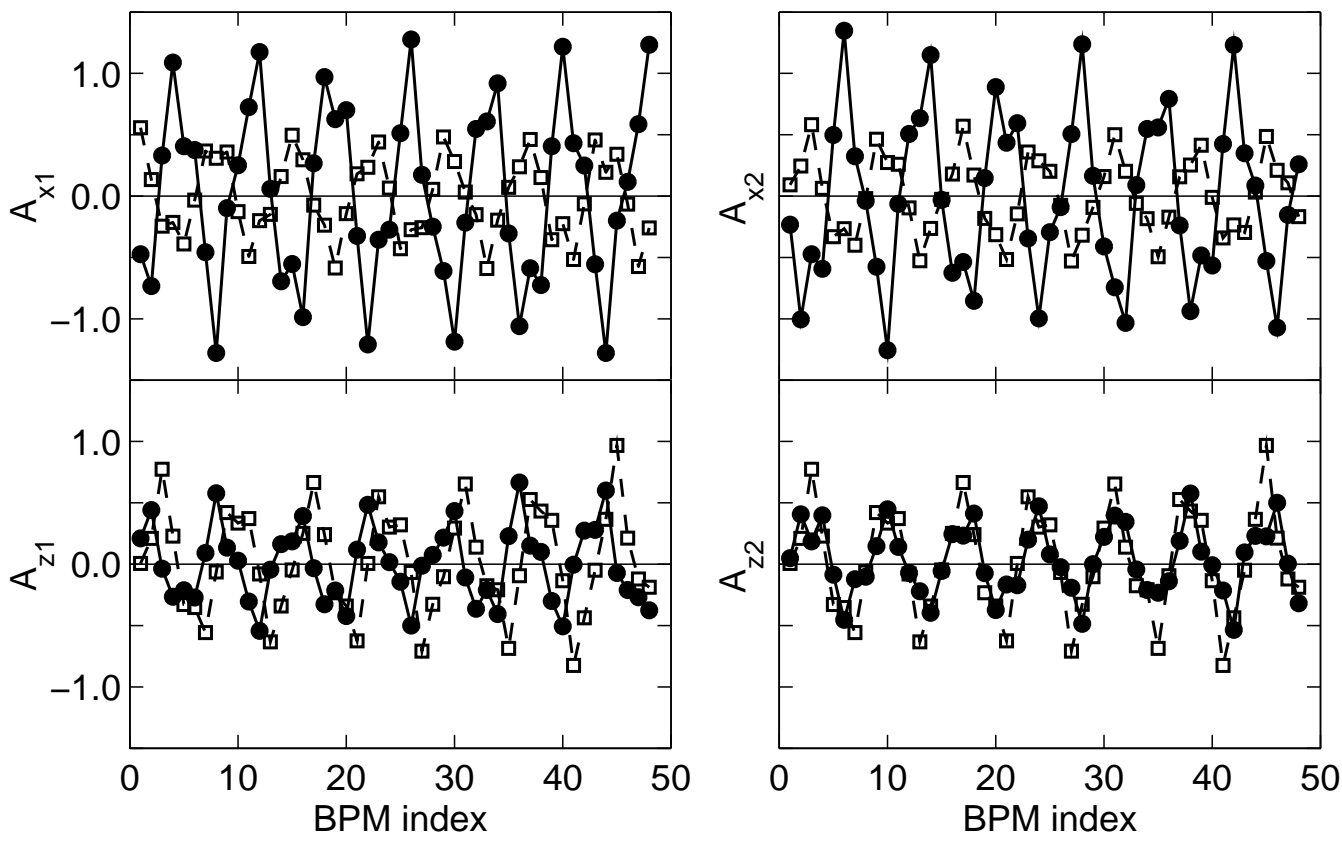

Figure 5.6: Spatial patterns of the betatron modes of AC data for the burst in turn 2060:2320. Top: spatial patterns of the two horizontal betatron modes. Bottom: spatial patterns of the two vertical betatron modes. Both horizontal BPMs (circles) and vertical BPMs (squares) are shown. Note there is strong linear coupling between the two transverse planes. 

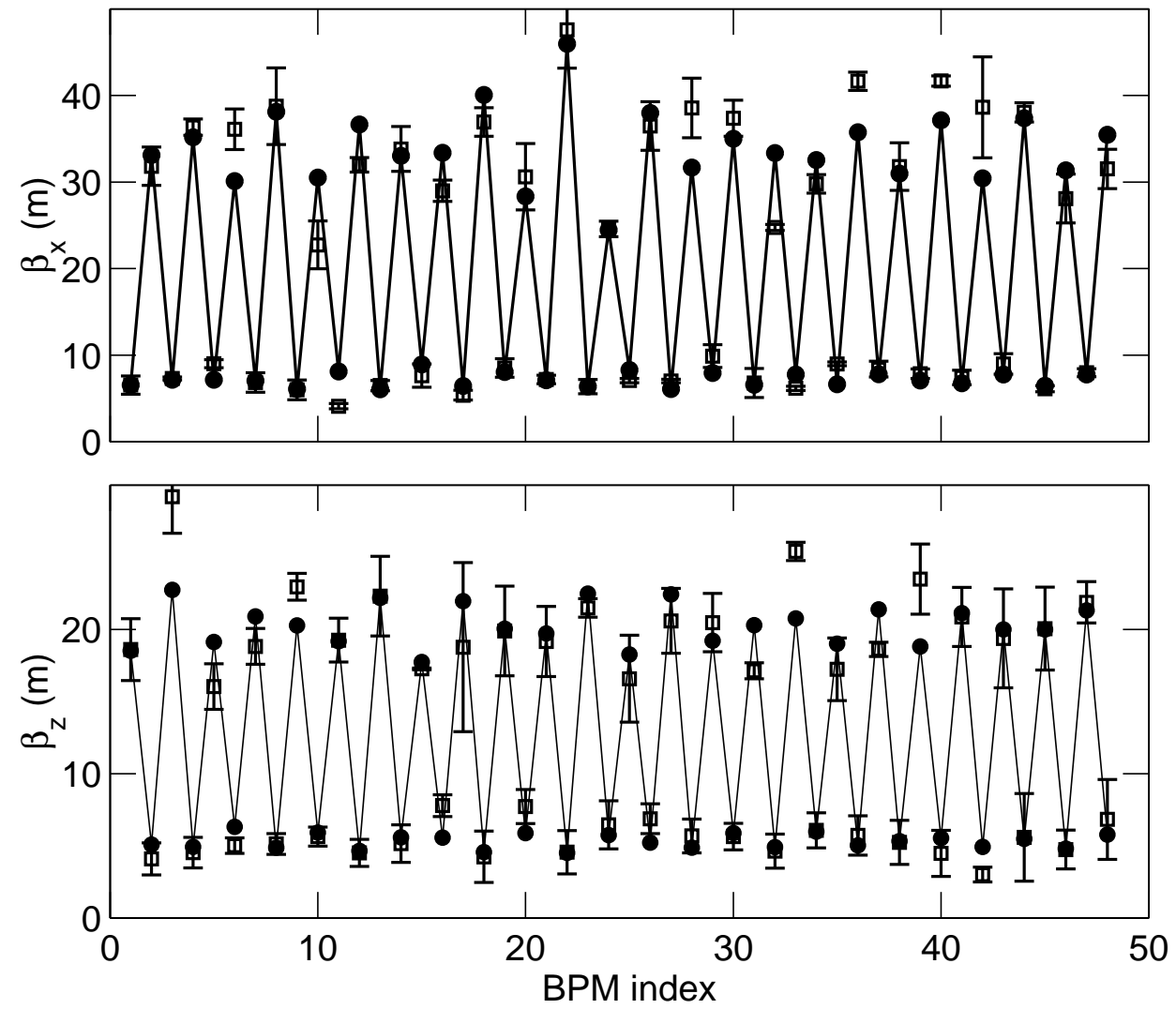

Figure 5.7: The measured beta functions (squares) are compared to model calculation (solid circles) for AC data in April 2004. Top: horizontal beta function. Bottom: vertical beta function. 
The betatron tunes for both transverse planes were derived from the coherent betatron modes for the entire Booster cycle. When beam energy is ramped up, the linear coupling is weakened and the vertical betatron tune is unavailable. The measured tunes are compared to model calculation in Fig. 5.8. The agreement between the measurement and the model is also an indication of the accuracy of the model.

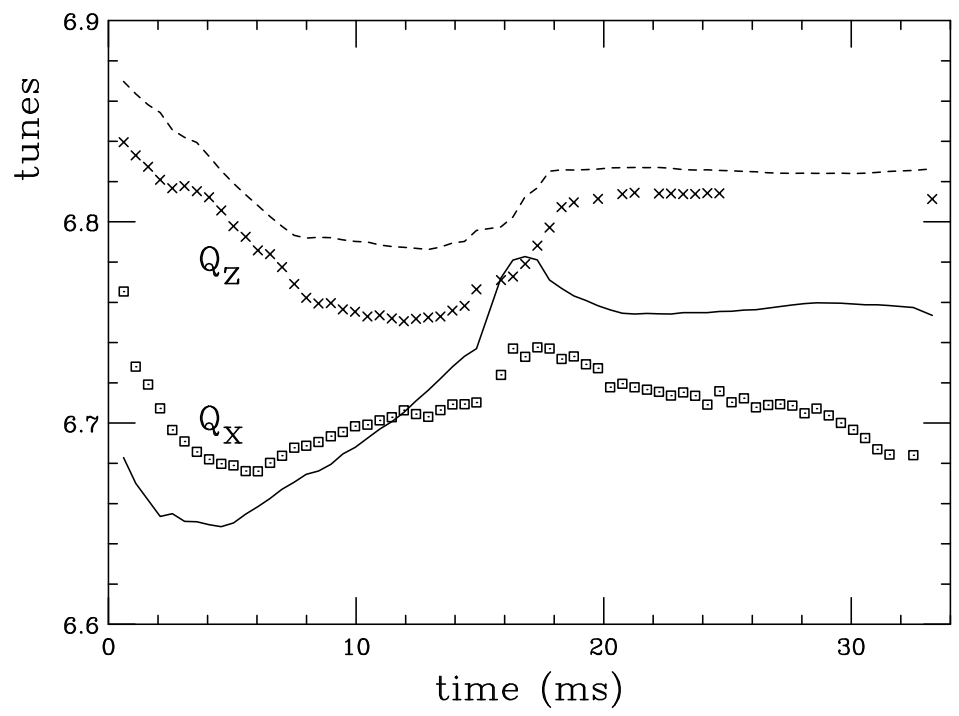

Figure 5.8: The horizontal (squares) and vertical (crosses) betatron tunes in a Booster cycle. Tunes calculated by MAD model are compared to measurements (solid and dash lines).

During the shutdown in fall 2004, the extraction dogleg in section L03 was also stretched out to reduce its perturbation to the lattice. The pinger was configured to kick the beam vertically. Below we show data taken in November 2004 after the shutdown. We set the pinger to be fired with $500 \mathrm{~Hz}$, i.e., every $2 \mathrm{~ms}$ so the burst has longer time to be damped out. The signals are relatively cleaner. The coherent 
betatron modes for the burst start at turn 620 are shown in Fig. 5.9, 5.10. Beta functions and phase advances for both transverse planes are measured. The results are shown in Fig. 5.11. The differences between the model and measurements are characterized by average $\Delta \beta / \beta$, which is $12 \%$ for both horizontal and vertical planes. The average differences of phase advances between the model and measurements are $0.10 \mathrm{rad}$ for the horizontal plane and $0.12 \mathrm{rad}$ for the vertical plane.

The lattice function measurements with turn-by-turn BPM data in different time and experimental setting indicate that the existing lattice model can predict beta function with an average error of $12 \%$ in $\Delta \beta / \beta$ and an error for phase advance from BPM to BPM of about $0.10 \mathrm{rad}$.

\subsection{Measurements of Transverse Impedance}

The betatron tunes in general depend on beam intensity because of the interaction between beam and the accelerator environment. The beam generates an electromagnetic field and the field acts back on the beam. The transverse effects of such interaction are betatron tune shifts and possibly transverse instabilities. The tune shift has an incoherent component which depends on positions of the particles in the beam and has no average effect. The incoherent tune shift only increases the tune spread of the beam and is not observable in BPM data because a BPM records only the motion of the beam centroid. However, the coherent component, which is the average tune shift of the beam, can be measured from BPM turn-by-turn data if the measurement has high accuracy. The ICA method increases accuracy of tune measurements by extracting a clean coherent betatron signal with information from all available BPMs and using advanced tune evaluation method such as the interpolated FFT [31]. We have measured the betatron tune shifts due to increased intensity in half of the Booster cycle and calculated the transverse impedance with the results. 

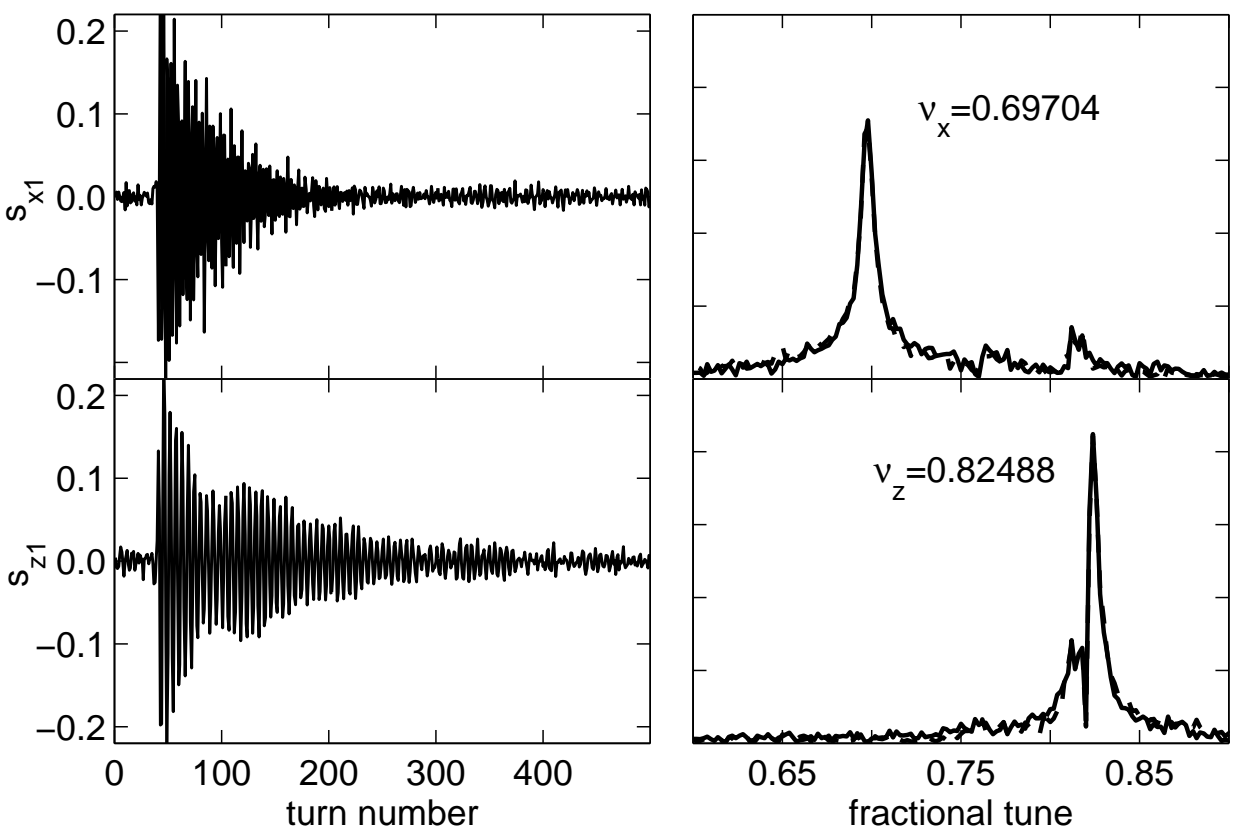

Figure 5.9: Examples of betatron modes of $\mathrm{AC}$ data taken in November 2004 when both doglegs were stretched. The burst corresponds to turns 620:1120. Top: temporal pattern (left) of a horizontal betatron mode and the FFT spectra (right) of the two horizontal modes. Bottom: temporal pattern (left) of a vertical betatron mode and the FFT spectra (right) of the two vertical modes. 

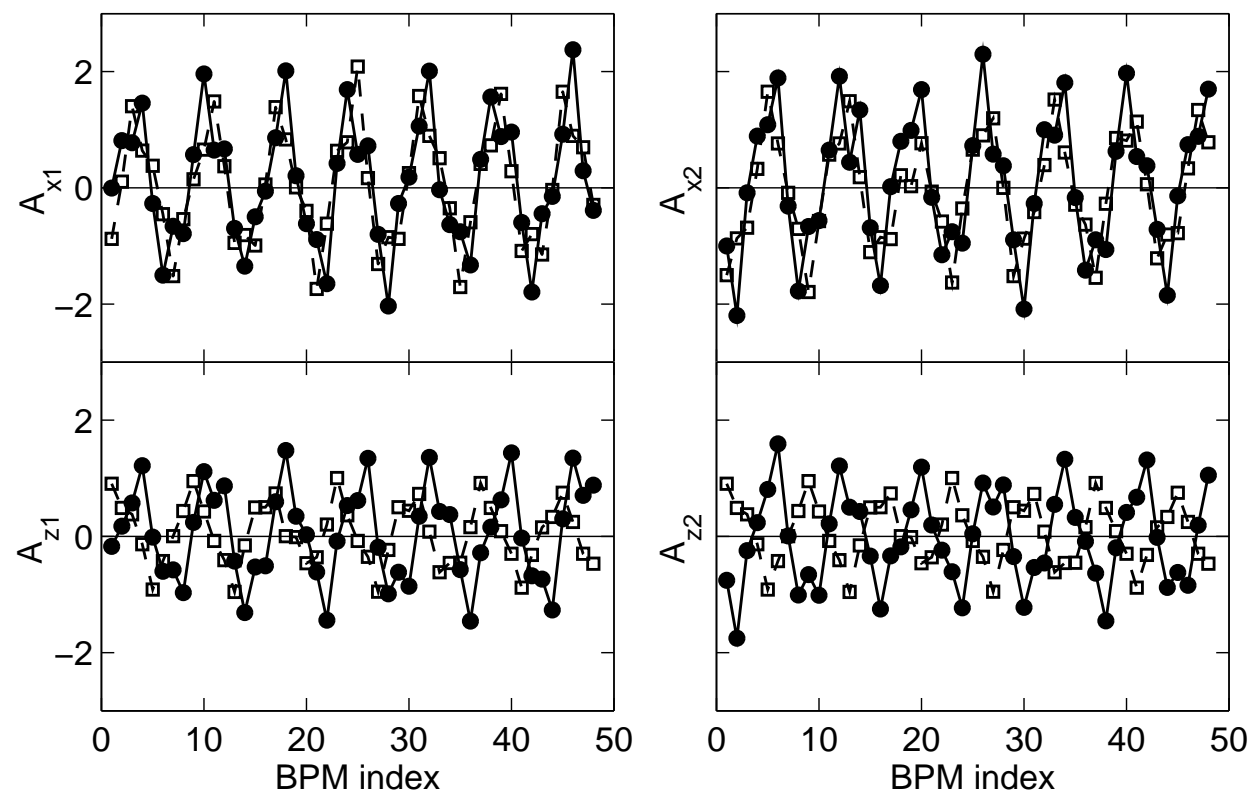

Figure 5.10: Spatial patterns of betatron modes of AC data with two re-positioned doglegs. Top: spatial patterns of the horizontal modes. Bottom: spatial patterns of the vertical modes. Horizontal BPMs (solid circles) and vertical BPMs (hollow squares) are both shown. Note the linear coupling is strong. 

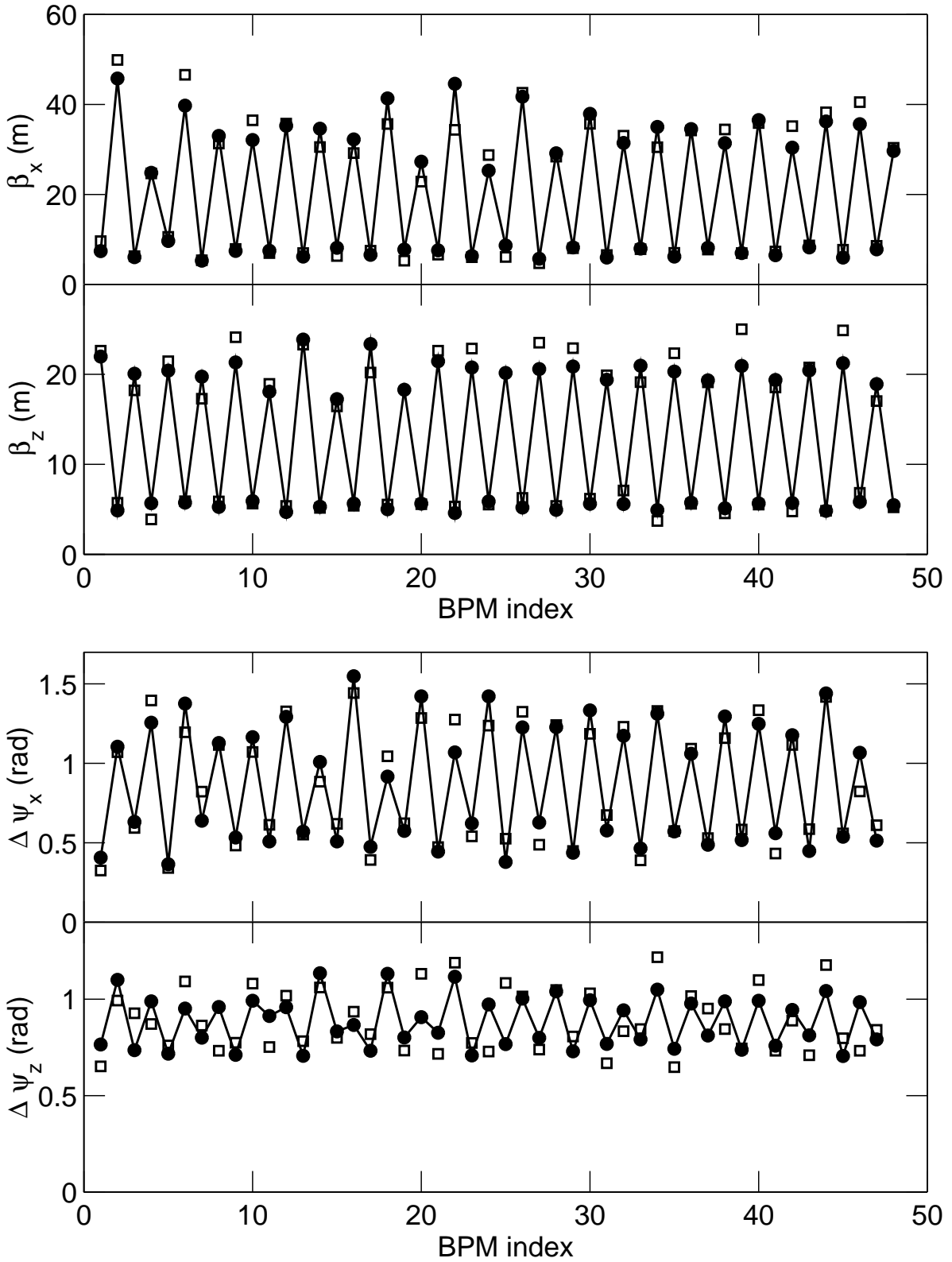

Figure 5.11: Top: the measured beta functions. Bottom: the phase advances between adjacent BPMs. Measurements (squares) are compared to model calculation (circles). 
The measured transverse impedance is compared to the calculations.

Turn-by-turn data were taken for different intensity levels corresponding to 2, 4, 6,8 and 10-turn injection. The pinger was set to be fired every $0.5 \mathrm{~ms}$ with $2.0 \mu \mathrm{s}$ pulse width. The pinger voltage was ramped from $0.6 \mathrm{keV}$ at injection to $3.8 \mathrm{keV}$ near extraction. The pinger kicks the beam vertically.

The vertical tunes before transition for all data sets are plotted in Fig. 5.12. After transition and in a particular location near turn 3120, the betatron signal has some temporal beating which corresponds to two or three peaks in the spectrum. Examples of beating betatron signal are shown in Fig. 5.13. The tunes cannot be uniquely determined from turn-by-turn signal in these regions. The cause of such behavior is not known. The impedance measurement is limited in regions where the vertical tune can be unambiguously obtained.

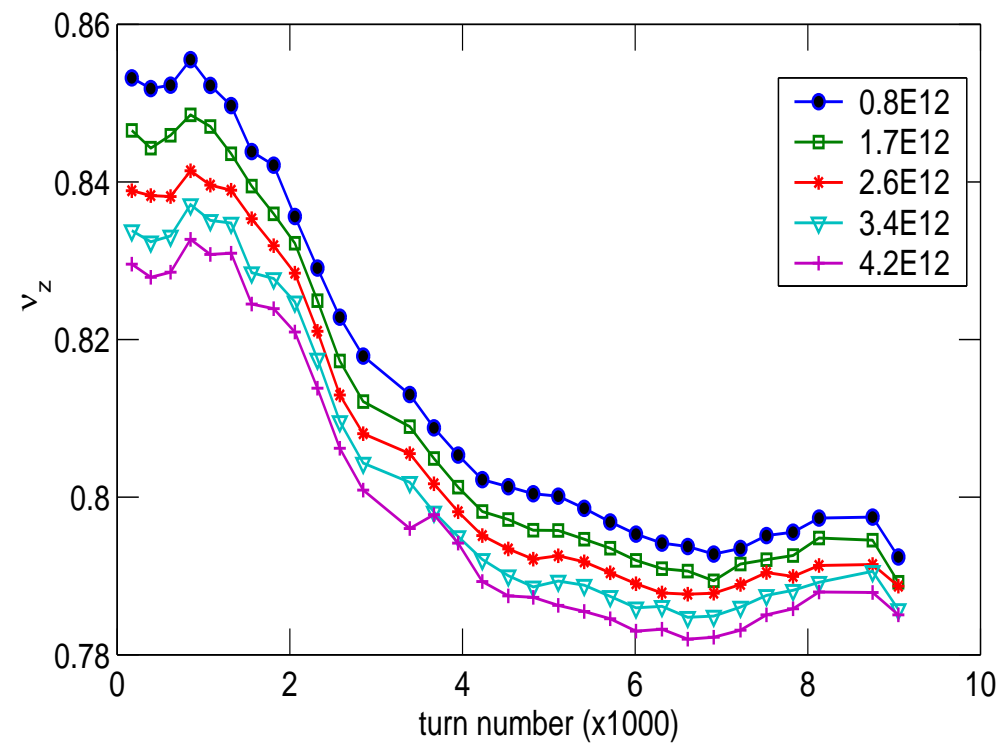

Figure 5.12: (Color) The vertical tunes before transition for various intensity levels. 

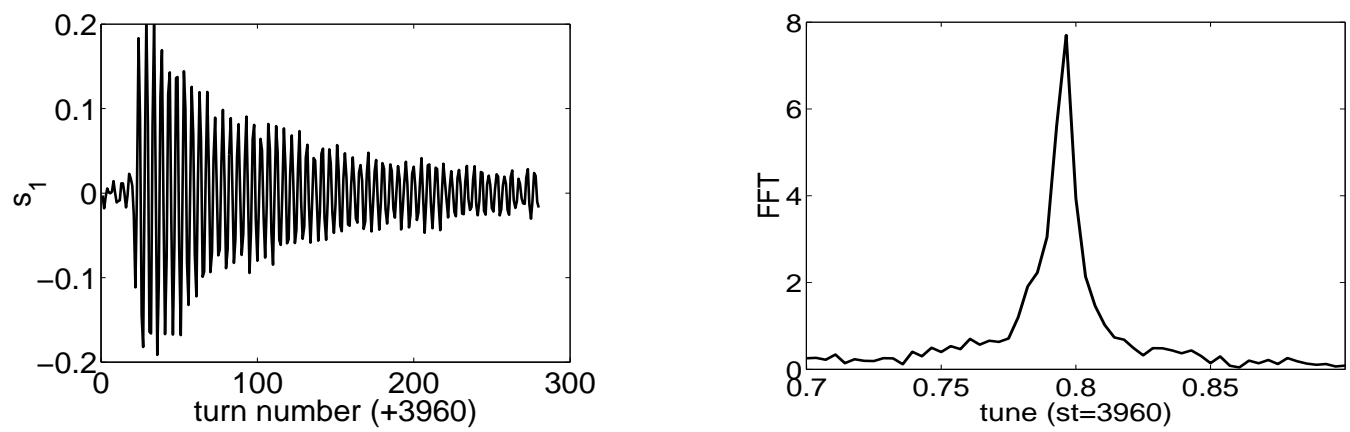

(a) typical before transition (the burst starting at turn 3960)
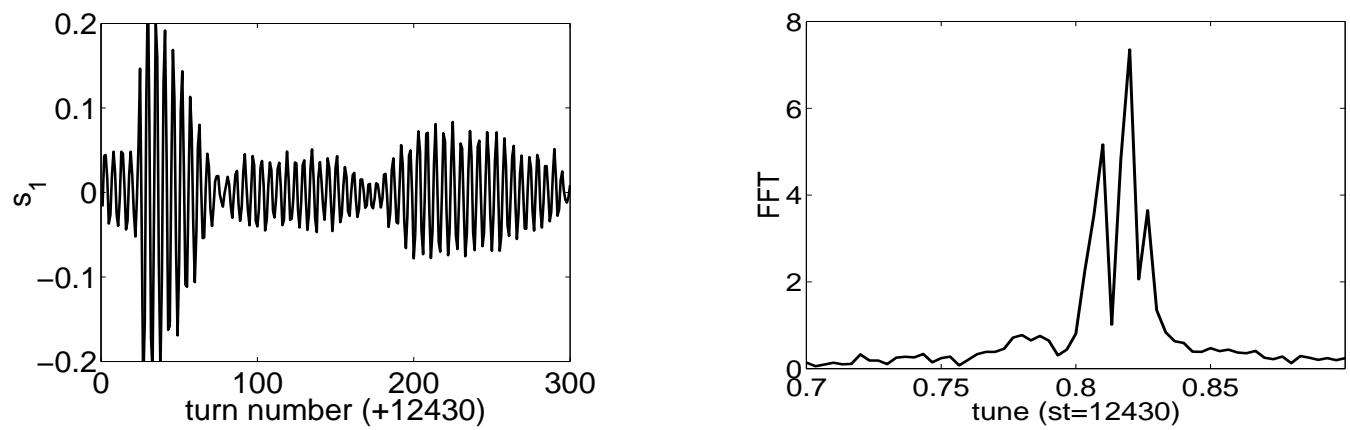

(b) typical after transition (the burst starting at turn 12430)
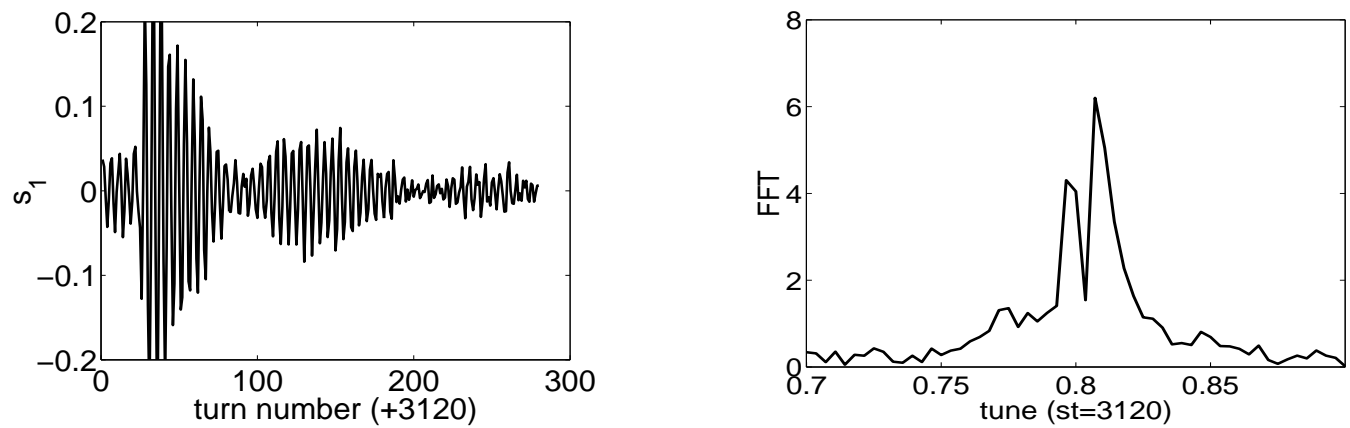

(c) a special case (at turn 3120) before transition.

Figure 5.13: Typical betatron modes before and after transition.

(a) The typical behavior before transition; (b) the typical behavior after transition; (c) the burst starting at 3120 turn is an exception of the typical behavior before transition. 
The betatron tune shift comes from the imaginary part of transverse impedance. The transverse equation of motion for the beam centroid is [1]

$$
\langle\ddot{y}\rangle+\left(\nu_{0} \omega_{0}\right)^{2}\langle y\rangle=\frac{F_{\perp}(t)}{\gamma m}, \quad F_{\perp}(t)=-j \frac{e \beta I Z_{\perp}}{2 \pi R}\langle y\rangle,
$$

where $\omega_{0}$ is angular revolution frequency, $R$ is average ring radius, $I$ is beam current, $Z_{\perp}$ is transverse impedance and the derivative is with respect to time. From Eq. (5.1) one can derive

$$
\left(\nu \omega_{0}\right)^{2}=\left(\nu_{0} \omega_{0}\right)^{2}+\frac{e \beta I\left(j Z_{\perp}\right)}{2 \pi \gamma m R}
$$

and hence

$$
\nu=\nu_{0}-\frac{1}{4 \pi} \frac{e \beta \operatorname{Im} Z_{\perp}}{\gamma m R \nu_{0} \omega_{0}^{2}} I
$$

The above result is for coasting beam. For bunched beam, a form factor that represents the longitudinal distribution of the beam needs to be included. The form factor for a Gaussian beam is $B_{f} / \sqrt{2}$ (see Eq. (6.210) of Ref. [43]), where $B_{f}$ is the bunching factor defined as the ratio of the peak current $\hat{I}$ to the average current $\bar{I}$, i.e.,

$$
\hat{I}=B_{f} \bar{I}, \quad \bar{I}=\frac{N e \beta c}{2 \pi R} .
$$

The depressed tune in Eq. (5.3) can be re-written as

$$
\nu=\nu_{0}-\frac{\operatorname{Im} Z_{\perp}}{Z_{0}} \frac{N r_{0}}{2 \pi \gamma \nu_{0}} \frac{B_{f}}{\sqrt{2}}
$$

where $Z_{0}=377 \Omega$ is vacuum impedance, $r_{0}=1.535 \times 10^{-18} \mathrm{~m}$ is the classical radius of proton and $N$ is the total number of protons in the ring. We can then fit the tunes with respect to intensity to a linear curve

$$
\nu=\nu_{0}+a N, \quad a=-\frac{\operatorname{Im} Z_{\perp}}{Z_{0}} \frac{r_{0}}{2 \pi \gamma \nu_{0}} \frac{B_{f}}{\sqrt{2}}
$$




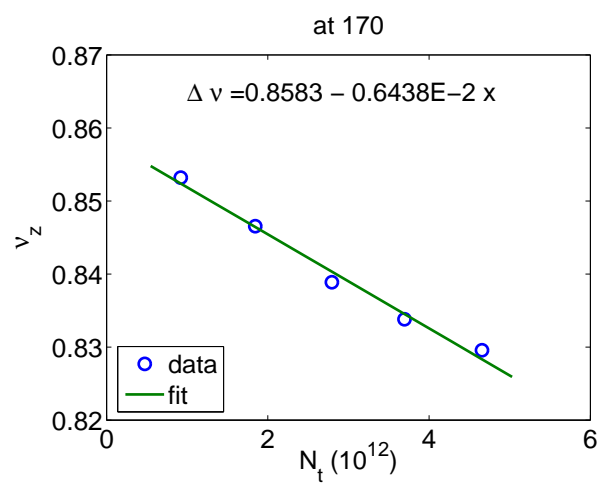

(a) st $=170$

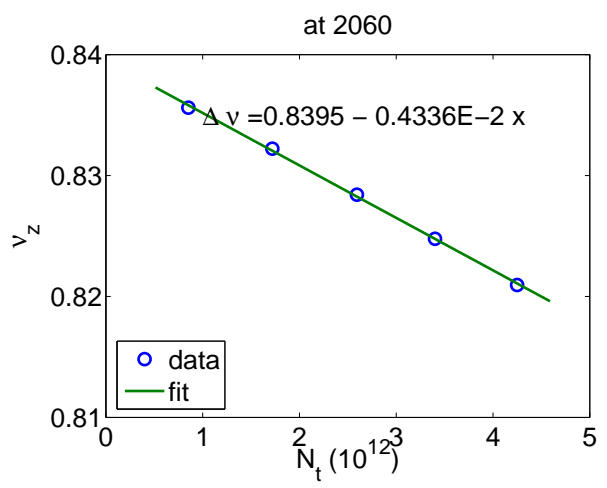

(c) st $=2060$

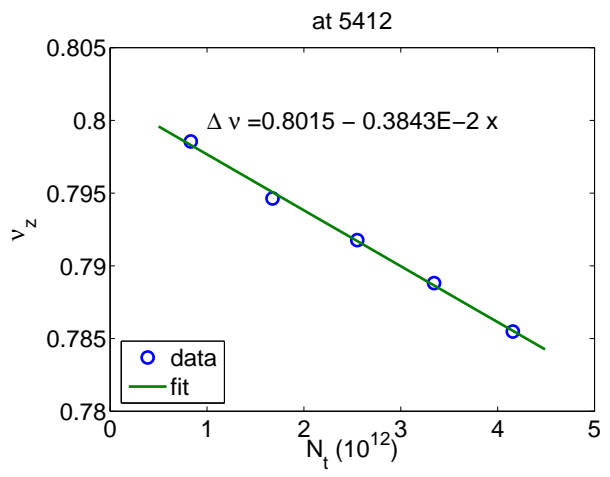

(e) st $=5412$

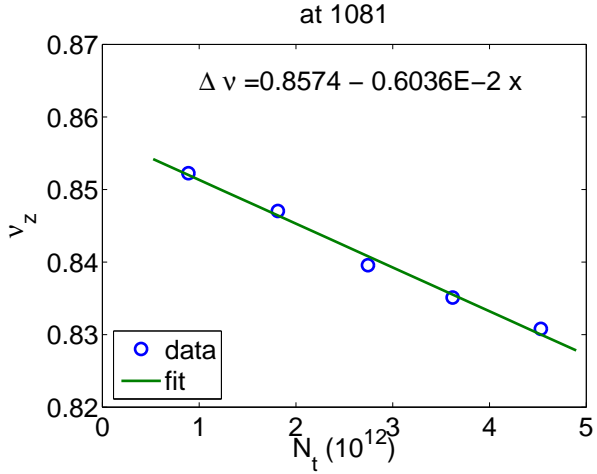

(b) st $=1081$

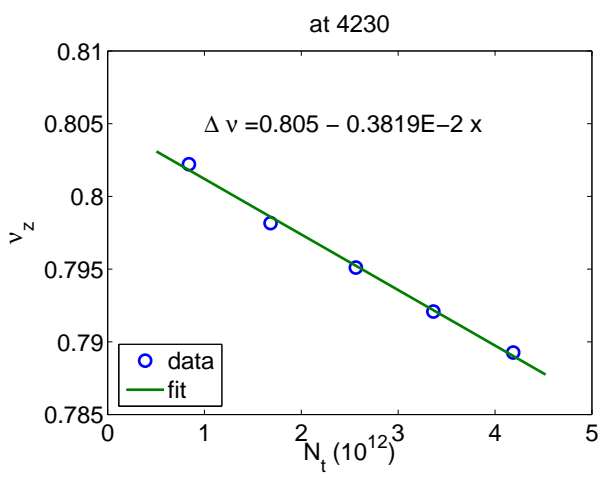

(d) st $=4230$

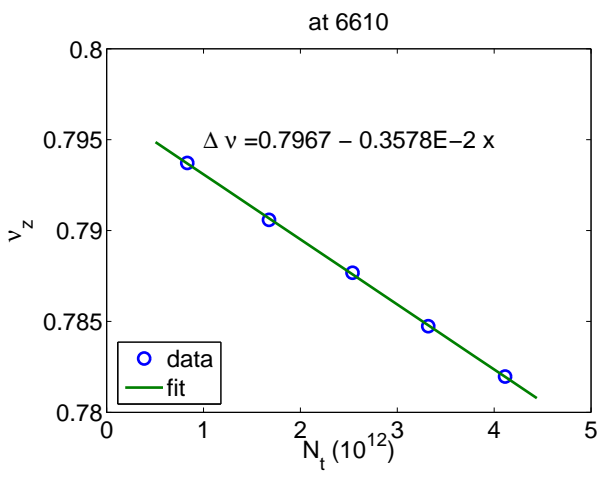

(f) st $=6610$

Figure 5.14: Fitting vertical betatron tune $\nu_{z}$ vs. total number of protons $N$ to linear curves. The fitting curves at different times before transition are shown. 


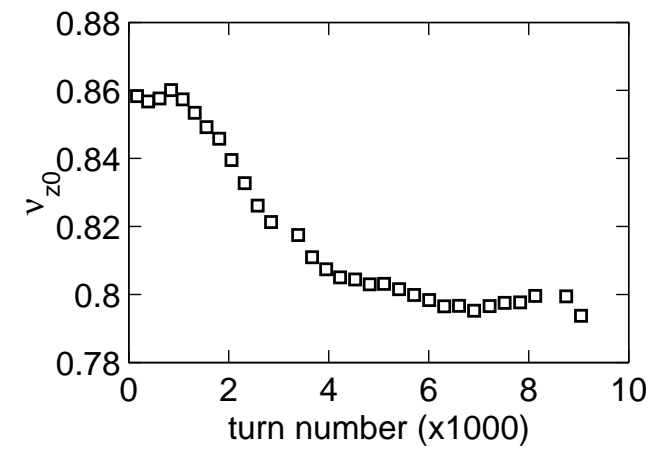

(a) $\nu_{0}$

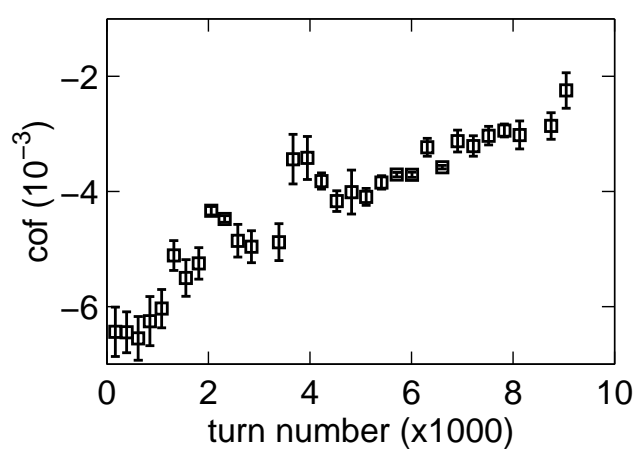

(b) $a$

Figure 5.15: The fitting parameters of vertical de-tuning for the model $\nu=\nu_{0}+a N$ with the number of protons $N$ in units of $10^{12}$. (a) The vertical tune for zero-charge beam, or $\nu_{0}$. (b) The parameter $a$, or the slope of $\Delta \nu_{z}$ vs. $N$. 
at each observation point of the cycle. Some fitting curves are shown in Fig. 5.14. The fitted parameters $\nu_{0}$ and $a$ are shown in Fig. 5.15.

The bunching factor has been obtained through bunch length measurements with beam current signal detected by the resistive wall monitor. The bunching factor is compared to calculation in Fig. 5.16. In the calculation the longitudinal emittance is assumed to be $0.08 \mathrm{eV} \cdot \mathrm{s}$. Using the bunching factor and the fitted slopes, the imaginary part of transverse impedance is derived with

$$
\operatorname{Im} Z_{\perp}=-2 \pi \gamma \nu_{0} \frac{\sqrt{2}}{B_{f}}\left(\frac{Z_{0}}{r_{0}}\right) a .
$$

The result is shown in Fig. 5.17. The value of $\operatorname{Im} Z_{\perp}$ is found to be nearly $50 \mathrm{M} \Omega / \mathrm{m}$ at injection and it decreases to about $15 \mathrm{M} \Omega / \mathrm{m}$ before transition.

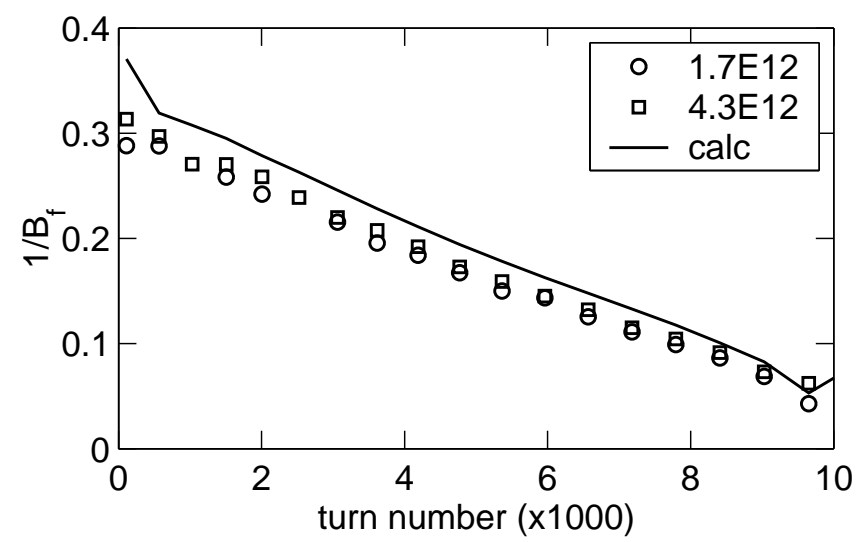

Figure 5.16: Inverse of the measured and calculated bunching factor $\left(1 / B_{f}\right)$ before transition. Measurements are under two intensity levels. The calculation is based on a $95 \%$ longitudinal emittance of $0.08 \mathrm{eV} \cdot \mathrm{s}$.

The transverse impedance comes from various sources, including image charge and image current in vacuum chamber and magnets, resistive wall impedance of the 


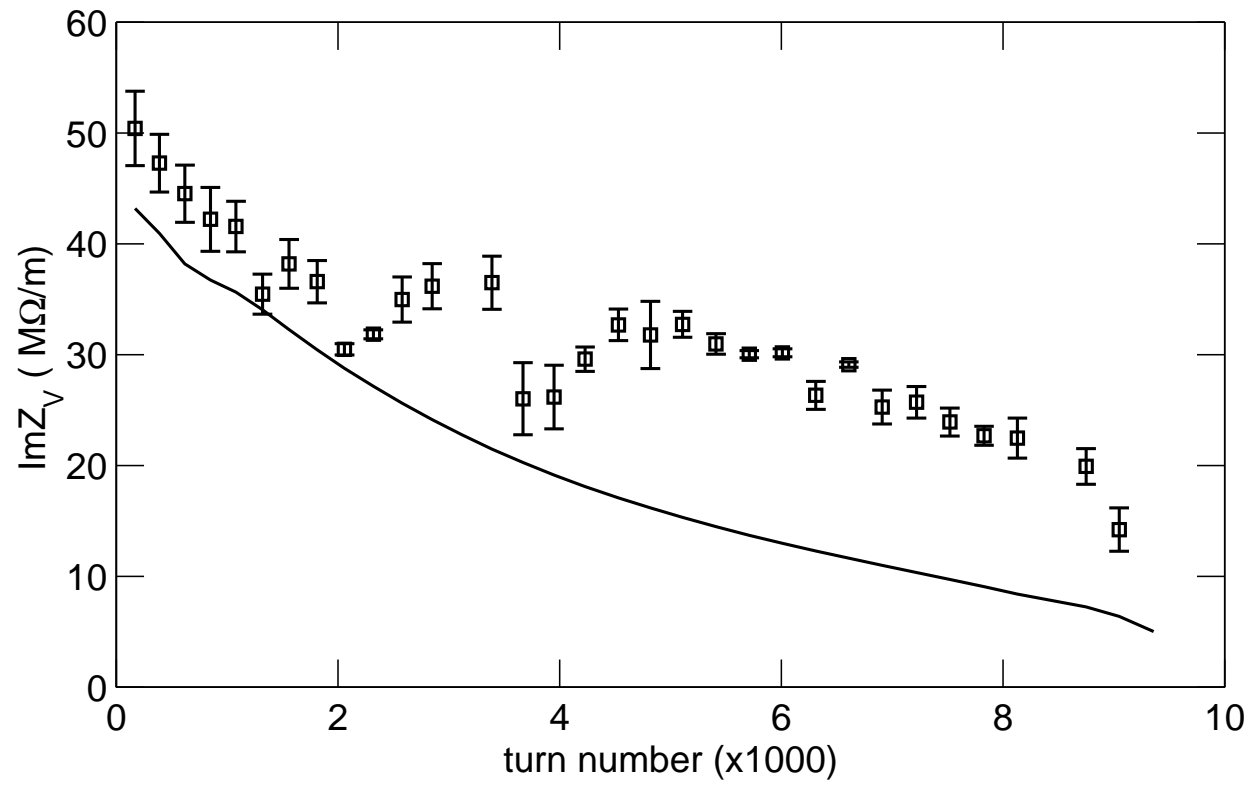

Figure 5.17: The measured imaginary part of transverse impedance $\operatorname{Im} Z_{\perp}$ (square) is compared to its calculated counterpart (solid line). The calculation considers Laslett tune shift due to image currents in magnet poles and image charge in vacuum pipe. 
vacuum chamber and magnets. It is estimated in Ref. [41] that the resistive vacuum chamber of the Booster contributes a very small fraction to the vertical transverse impedance by

$$
Z_{V}(\omega)=(1+j) \frac{0.122}{\sqrt{\omega / \omega_{0}}} \mathrm{M} \Omega / \mathrm{m} .
$$

The resistive wall impedance from the magnet poles which covers $59 \%$ of the ring should also be negligible because the magnets are enclosed with a steel shell which bypasses the surface current. The coherent Laslett tune shift due to the magnetic field and the electric filed is [42]

$$
\Delta \nu_{z}=-\frac{N r_{0} R}{\pi \gamma \nu_{z 0}}\left[\left(\frac{B_{f}}{\gamma^{2} \beta^{2}}+1\right)\left\langle\frac{\xi_{e}}{h^{2}}\right\rangle+\left\langle\frac{\xi_{m}}{h_{m}^{2}}\right\rangle\right]
$$

where $\langle\cdots\rangle$ denotes average over the entire ring, $\xi_{e}$ and $\xi_{m}$ are tune shift coefficients of electric filed and magnetic field, respectively, $2 h$ and $2 h_{m}$ denote the vacuum pipe diameter or magnet pole gap distance. The magnetic field term represents the image current in magnet poles. Considering the different geometry of the F and D magnet, we get

$$
\left\langle\frac{\xi_{m}}{h_{m}^{2}}\right\rangle=\frac{\xi_{m}}{2 \pi R}\left(\frac{L_{F}}{h_{F}^{2}}+\frac{L_{D}}{h_{D}^{2}}\right) \times 48=636.7 \mathrm{~m}^{-2},
$$

using $L_{F}=L_{D}=2.889 \mathrm{~m}, h_{F}=2.08 \mathrm{~cm}, h_{D}=2.86 \mathrm{~cm}$ and $\xi_{m}=\pi^{2} / 16$ for parallel plates. The electric field term is

$$
\left\langle\frac{\xi_{e}}{h^{2}}\right\rangle=\frac{\xi_{e}}{2 \pi R} \sum_{\text {sections }} \frac{L}{h^{2}}=227.4 \mathrm{~m}^{-2}
$$

using $\xi_{e}=1 / 2$ for circular pipe and that the total length of vacuum pipe with radius $2.08 \mathrm{~cm}$ (Long sections and mini straight sections) is $168 \mathrm{~m}$ and the length with radius $5.40 \mathrm{~cm}$ is $28.8 \mathrm{~m}$ (Short sections). The transverse impedance corresponding to Eq. (5.9) can be estimated by comparing it to Eq. (5.5). The result is compared to the measurement in Fig. 5.17. It is seen that the calculation does not completely explain 
the measured impedance. However, the order of magnitude and the trend within the cycle are in good agreement. Other sources could also contribute to the impedance.

\subsection{Chromaticity Measurements}

The high accuracy of betatron tune measurements through the application of the ICA method to turn-by-turn BPM data also provides a convenient way to measure the chromaticity. For the Booster, this can be done by changing the reference radial orbit (i.e., ROF curve) and measuring the corresponding change of betatron tunes. The momentum deviation introduced by changes of the ROF curve is measured by comparing the average orbit shift to the dispersion function calculated by the lattice model.

We carried out such measurements in June 2005. In the experiment the Booster was in regular ramping cycle. The pinger kicks vertically every $1.0 \mathrm{~ms}$, corresponding to burst lengths of 500 to 600 turns. The ROF curve was shifted from one side of the vacuum chamber to the middle and then to the other side while turn-by-turn data were taken at all three positions. The total change of radial orbit was $5 \mathrm{~mm}$ without significant beam loss at both limits. The beam intensity was $0.8 \times 10^{12}$ protons per cycle (2-turn injection). Two data sets were taken, one with the chromaticity sextupoles in short sections (SEXTS) off and the other with SEXTS on. The long section chromaticity sextupoles (SEXTL) were kept on.

The measured tunes are shown in Fig. 5.18. The horizontal tunes can be measured only before $12 \mathrm{~ms}$ in the cycle while there is still considerable linear coupling. The momentum deviation is estimated using

$$
\delta=\left\langle\frac{\Delta x}{D}\right\rangle
$$

where $D$ is the dispersion function obtained from model calculations, $\Delta x$ is the radial 
orbit shift and the sum is over the horizontal BPMs. Taking the middle radial position as the reference, the orbit shift at either inward or outward limit corresponds to momentum deviation of about $\pm 1.2 \times 10^{-3}$ with small variations depending on where it is in the cycle. Fig. 5.18 indicates a clear dependence of the betatron tunes on momentum deviation. The chromaticity, or the slope of betatron tune over momentum deviation can be obtained by a linear fitting with the three data points at each observation point. The results are shown in Fig. 5.19. The error bar of chromaticity measurements is estimated to be $\Delta C= \pm 0.5$, assuming the accuracy of tune measurements is \pm 0.001 and using the fact that we changed momentum deviation by more than $2 \times 10^{-3}$. Chromaticities calculated by the lattice model throughout the cycle using experimental settings are plotted in Fig. 5.20. The natural chromaticities are $C_{z}^{\text {nat }} \approx-7.1$ and $C_{x}^{\text {nat }} \approx-9.2$ for the entire cycle. The chromaticity sextupoles (SEXTL and SEXTS) partially compensate them. The currents of the chromaticity sextupoles in the cycle when data were taken are shown in Fig. 5.21. The effect of the SEXTS sextupoles can be seen from the differences between the dashed and the solid lines in Fig. 5.20. It is also experimentally observed in the chromaticity measurements in Fig. 5.19. The measured horizontal chromaticity reduction due to SEXTS is compared to model calculation in Fig. 5.22. The descending trend in the figure is specified by both the SEXTS current $I_{\text {SEXTS }}$ and the ramping momentum $P$. At $400 \mathrm{MeV}, \Delta I_{\mathrm{SEXTS}}=1 \mathrm{~A}$ causes a change of horizontal chromaticity of $\Delta C_{x}=0.47$, which is consistent with the calculation using Eq. (2.52).

Chromaticities were also measured when both sextupole families were turned off. The results of two measurements in different time (July 29 and August 25, 2005) are shown in Fig. 5.23. In the July 29 measurement, SEXTS was turned off only for the first $12 \mathrm{~ms}$. After that the current $I_{\text {SEXTS }}$ steadily varies from -34 A to -100 A, with $I_{\text {SEXTS }} P(t=0) / P(t) \approx-10 \mathrm{~A}$. It is noted that the chromaticity measurements in ramping cycles (AC mode) presented above do not agree to an earlier measurement 

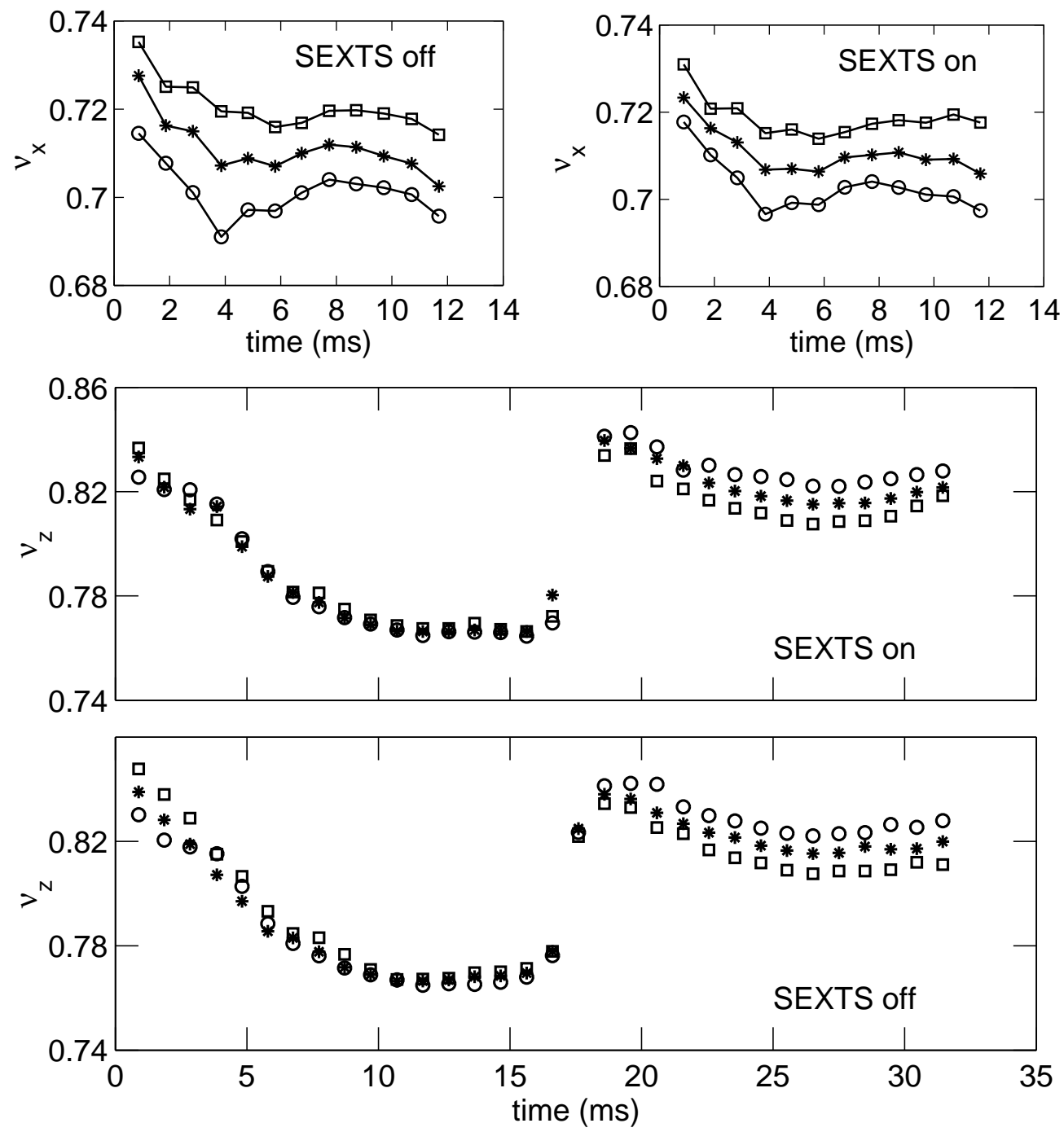

Figure 5.18: The betatron tunes of the chromaticity measurement. Top : horizontal tune $\nu_{x}$ when SEXTS is on or off. Middle and bottom: vertical tune $\nu_{z}$ when SEXTS is on or off. The three curves represent radial beam position at the outward limit (squares), the center (stars) and the inward limit (circles). 


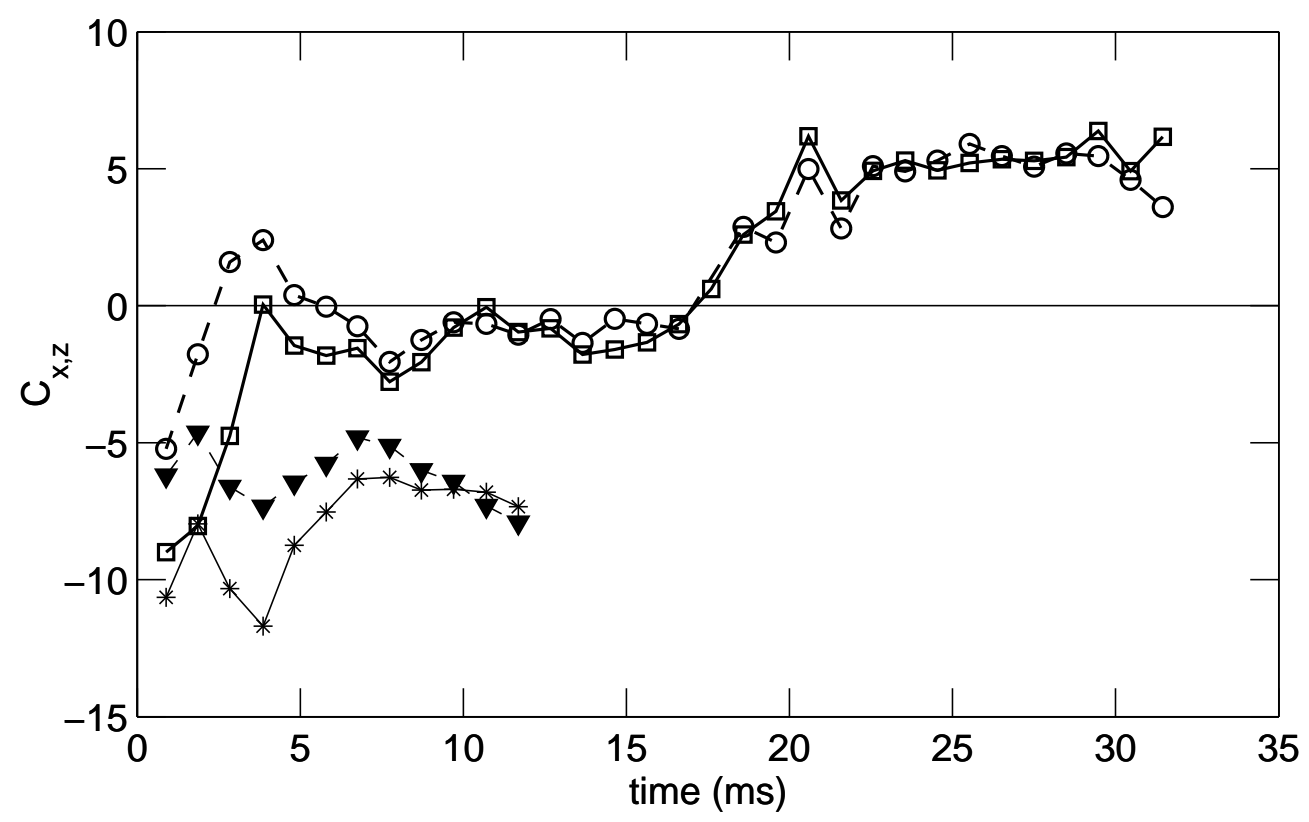

Figure 5.19: The measured horizontal chromaticity $C_{x}$ when SEXTS is on (triangles) or off (stars), and the measured vertical chromaticity $C_{z}$ when SEXTS is on (dash, circles) or off (solid, squares). The error bar is estimated to be \pm 0.5 . 

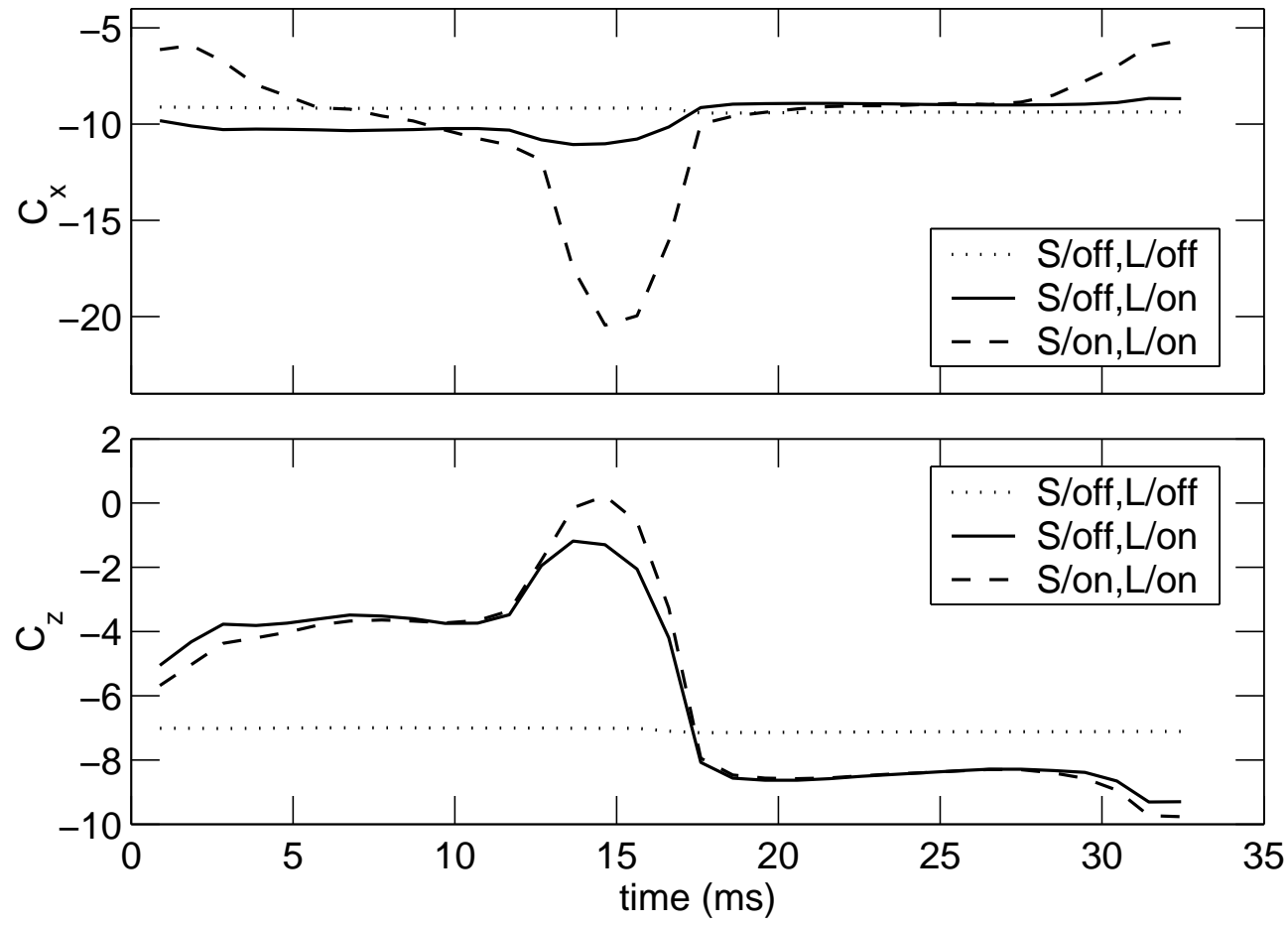

Figure 5.20: Chromaticities calculated with the lattice model using experimental settings. Top: horizontal chromaticity $C_{x}$. Bottom: vertical chromaticity $C_{z}$. The natural chromaticities (dotted), chromaticities with SEXTL on but SEXTS off (solid) and with both SEXTL, SEXTS on (dashed) are compared. 


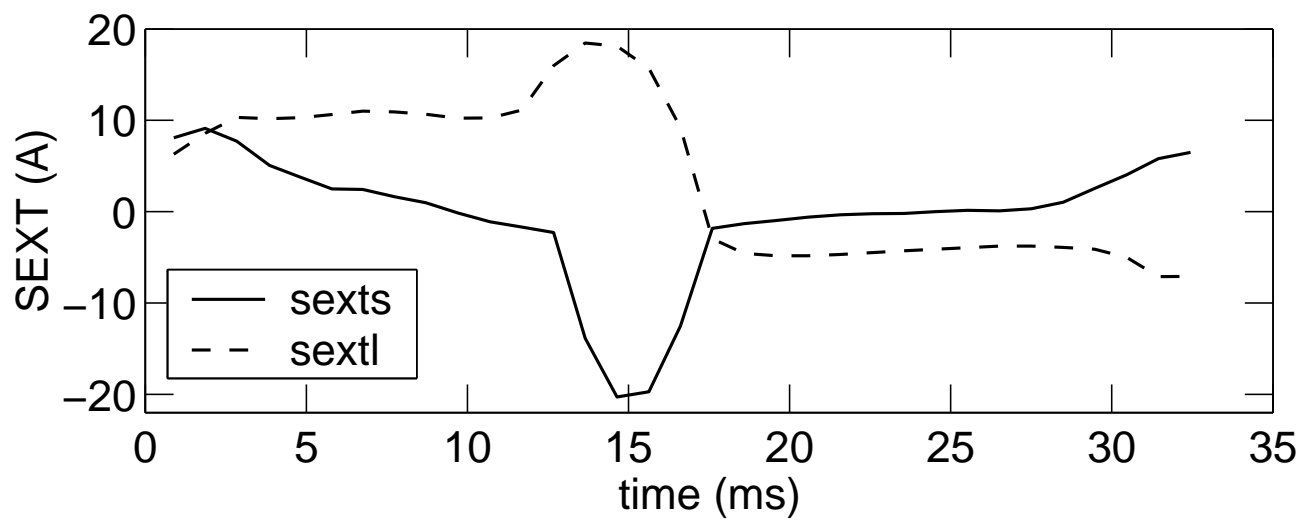

Figure 5.21: The currents of SEXTL and SEXTS in the cycle (for the June 2005 data), corrected to $400 \mathrm{MeV}$ equivalence by multiplying the factor $P(t=0) / P(t)$.

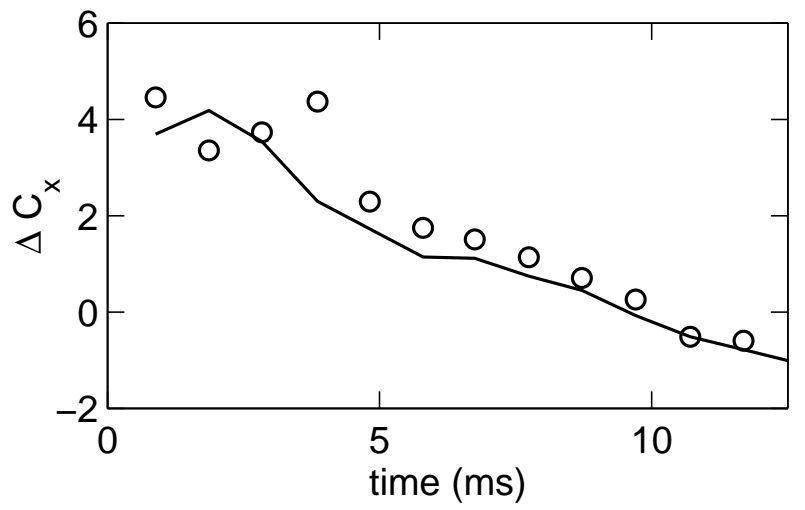

Figure 5.22: Horizontal chromaticity reduction $\Delta C_{x}$ due to SEXTS sextupoles. Model calculation (solid line) is compared to measurement (circle). 


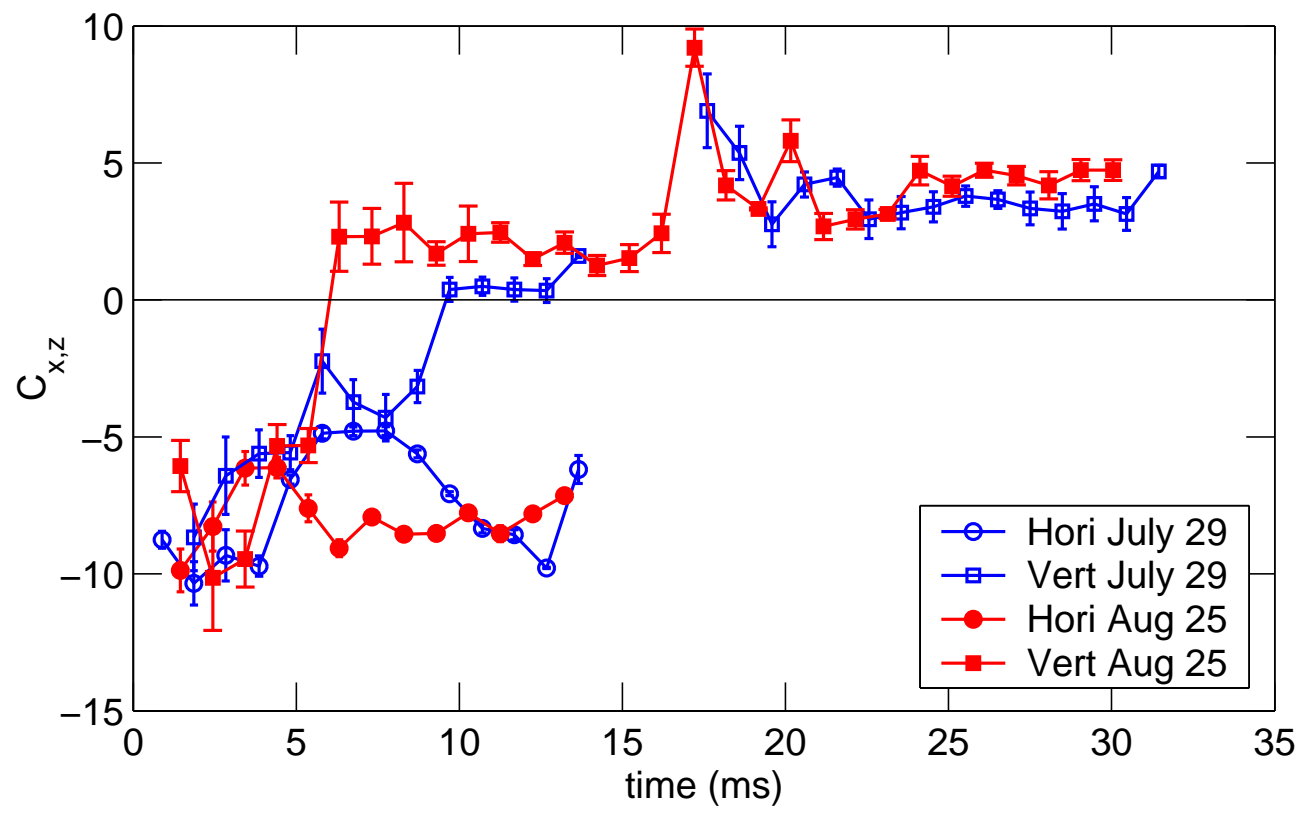

Figure 5.23: (Color) Horizontal and vertical chromaticities measured in AC cycles when both SEXTL, SEXTS sextupoles were turned off. Note SEXTS was turned off only for the first $12 \mathrm{~ms}$ for July 29 measurement. 
Table 5.1: Chromaticity measurement in DC cycles [38].

\begin{tabular}{|c|c|c|c|c|}
\hline Index & SEXTL(A) & SEXTS $(\mathrm{A})$ & $C_{x}$ & $C_{z}$ \\
\hline 1 & 0 & 0 & -21.7 & 11.6 \\
2 & 10 & 10 & -10.7 & 7.7 \\
3 & -10 & -10 & -33.7 & 15.1 \\
4 & 0 & -10 & -33.1 & 13.2 \\
\hline
\end{tabular}

which was conducted in fixed energy cycles (400 MeV, DC mode) [38]. The result of the DC measurement is listed in Table 5.1. Most noticeably the vertical chromaticity $C_{z}$ is positive in DC measurement and negative in the beginning of a ramping cycle (where the beam energy is also $400 \mathrm{MeV}$ ). The magnitude of $C_{x}, C_{z}$ in DC measurement is also much larger than the $\mathrm{AC}$ measurement and the natural chromaticity of the Booster. Table 5.1 shows that a change of SEXTS current of 1 A can raise the $C_{x}$ by 1.14 in $400 \mathrm{MeV}$, which is about twice of the value calclated with the lattice model and magnet specification. Since the DC measurement was done two years ago, we may need to re-do the measurement to verify that the machine status has not changed, even though we think there was no upgrade that could have changed the chromaticities much since then.

The ICA method for chromaticity measurement remarkably increases the accuracy and reduces the need of human interaction with the console. The chromaticity in the entire cycle is obtained in one shot, which saves a tremendous amount of time.

\subsection{Observation of Synchrotron Motion}

In turn-by-turn BPM data of ramping cycles, we have observed the synchrotron motion. The synchrotron signal is usually very weak because we didn't excite synchrotron 
motion intentionally ${ }^{2}$. Unlike what we have predicted in chapter 4.2 .2 , there are not only one but two or more synchrotron modes. Their spatial patterns often do not resemble the dispersion function. Even though the Booster's dispersion functions are all positive, the spatial patterns crosses zero. Figure 5.24 shows the spatial and temporal functions of two synchrotron modes from turn 3001 to turn 3400. The behavior of the spatial pattern of synchrotron modes is a consequence of the energy variation from BPM to BPM in one revolution. And such energy variation could come from (1) beam energy gain and loss by the longitudinal damper or (2) mis-matched ramping curves of the energy gain in rf cavities and the dipole fields. The momentum deviation is estimated to be about $5 \times 10^{-5}$ corresponding to energy deviation of $50 \mathrm{keV}$. This agrees well with a recent estimate of the one-turn energy loss of about $60 \mathrm{keV}$ [40]. Since the beam energy changes rapidly by about $340 \mathrm{keV}$ in one revolution, each BPM can see different momentum deviation. Figure 5.27 shows the spatial function divided by the dispersion function. This can be thought of as the amplitude of the off-momentum coordinate at each BPM location.

To better understand the synchrotron modes, we define the mode function

$$
\Delta p / p(s, t)=\frac{1}{D(s)}\left(A_{s 1}(s) s_{1}(t)+A_{s 2}(s) s_{2}(t)\right),
$$

where $D(s)$ is the dispersion function calculated with the model. The function $\Delta p / p(s, t)$ from turn 3001 to 3050 is shown in Fig. 5.26, where each square box corresponds to one turn in Booster. Note that the mode function has an energy deviation amplitude on the order of $50 \mathrm{keV}$, while the one turn energy gain at turn 3001 is about $340 \mathrm{keV}$, i.e. the average energy gain of each $\mathrm{RF}$ cavity is about $20 \mathrm{keV}$ or $40 \mathrm{keV}$ per pair.

The synchrotron tune throughout the cycle has been derived from the temporal patterns of the synchrotron modes. The result is compared to calculation in Fig. 5.25.

\footnotetext{
${ }^{2}$ The radial orbit is controlled by a feedback system. The rf phase cannot be suddenly changed.
} 


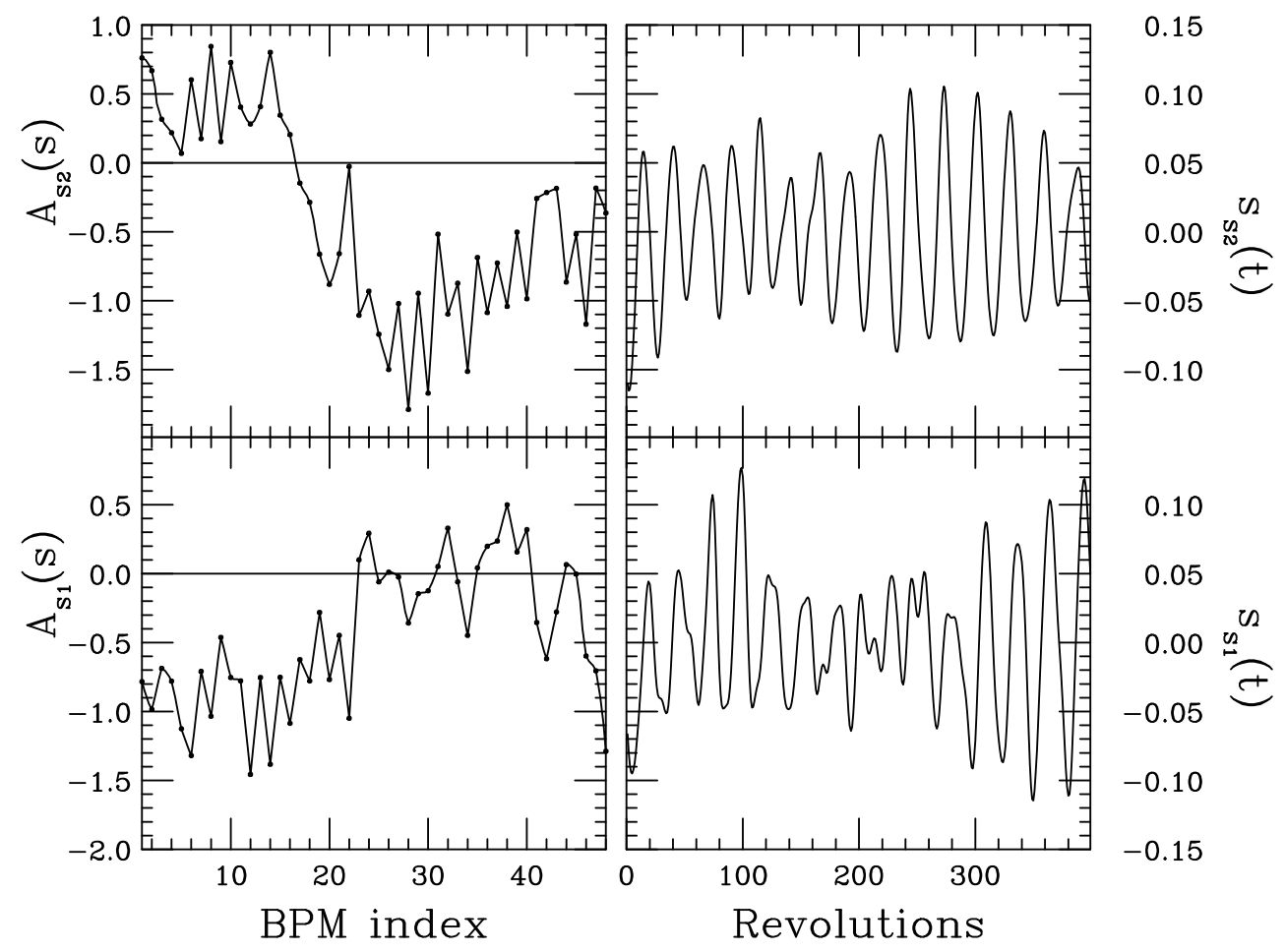

Figure 5.24: Examples of synchrotron modes in the Booster ramping cycle. The turn range is 3001 to 3400 (see Fig. 5.26). 
In the calculation the total rf voltages $V$ is based on typical recorded operation values (i.e., the RFSUM curve) and the synchronous phase is derived from the RFSUM and the ramping momentum curve of the Booster. The synchronous phase derived this way is smaller than the actual value in the beginning of the cycle. So the calculated synchrotron tune is larger than the measurement in this region as shown in Fig. 5.25.

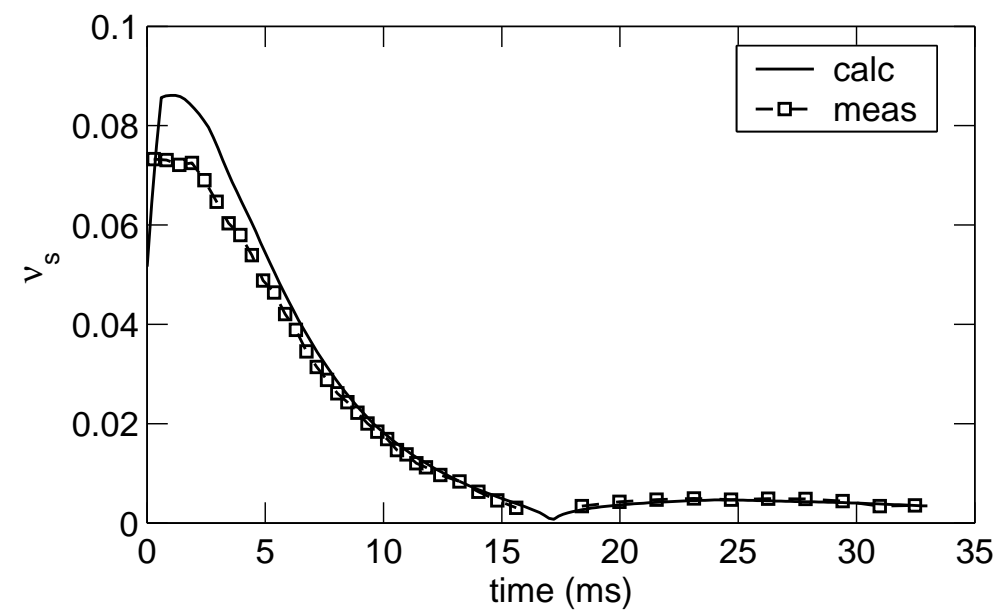

Figure 5.25: The measured synchrotron tune in a Booster cycle is compared to calculation. The discrepancy near injection comes from the incorrect synchronous phase used in calculation.

\subsection{Summary}

In this chapter we described the application of the ICA method to turn-by-turn data taken with the Fermilab Booster. It is used to measure the linear lattice functions in constant-energy mode (DC mode) and in regular ramping mode (AC mode). For both cases, moderate agreement between the existing model and the measurement is found. 


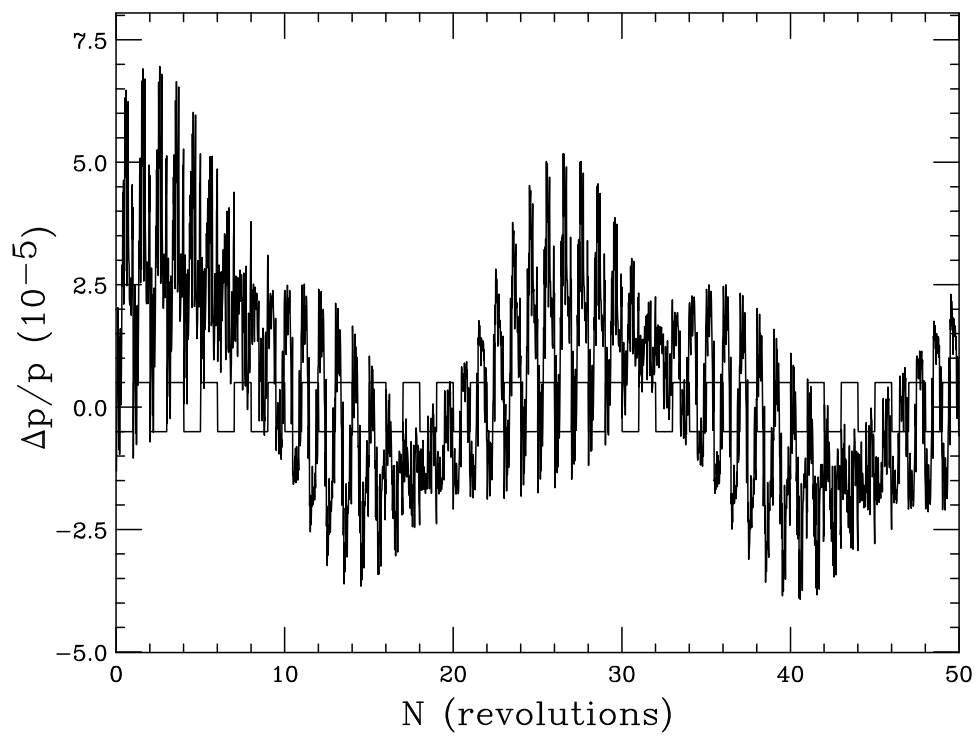

Figure 5.26: The variation of momentum deviation from BPM to BPM for 50 turns starting at turn 3001. Each square box represents one revolution. 


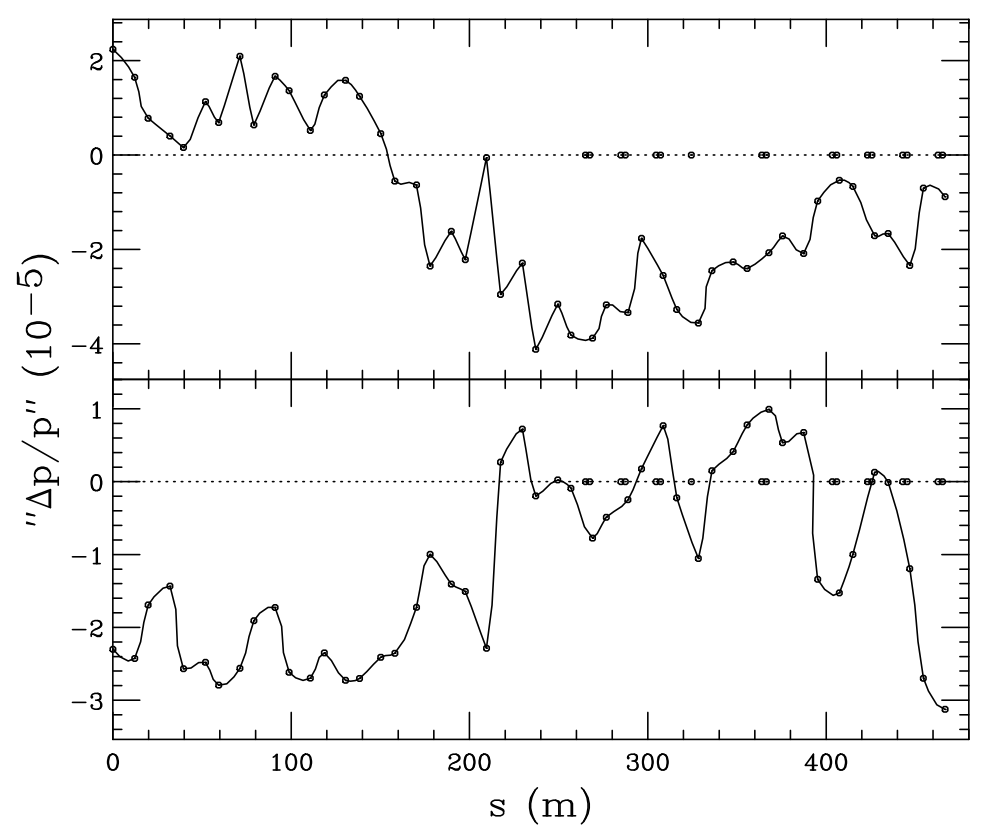

Figure 5.27: The spatial function of the synchrotron modes shown in Fig. 5.24 divided by the dispersion function. The resulting function can be thought of as the amplitude of the off-momentum deviation at each BPM location. The locations of rf cavities are shown as dots on the horizontal axis. 
The difference is about $12 \%$ in terms of average $\Delta \beta / \beta$. Using the high-accuracy tune measurement capability of the ICA method, we have measured the dependence of the betatron tunes on the beam intensity and beam momentum deviation, from which we derived the transverse impedance of the Booster and also the chromaticity. The synchrotron motion is also observed in turn-by-turn BPM data. The small energy variation between the BPMs can be seen in the spatial patterns of the synchrotron modes.

Since BPM turn-by-turn data is very easy to take and useful information can be derived from it with the ICA method, we can expect this method to find applications in other synchrotrons. For example, interest of using ICA has grown in RHIC and Tevatron studies. 


\section{Chapter 6}

\section{Emittance Growth}

In this chapter emittance data measured by the Booster ionization profile monitor (IPM) are studied. In order to analyze these data, we built the lattice model with experimental settings of ramping parameters to calculate the lattice functions. We studied the vertical emittance growth behavior in different stages of a Booster cycle and its relation to the beam intensity. The transverse and longitudinal components in the horizontal beam width are separated by a fitting model which makes use of the different scaling rules of basic accelerator physics. From these separated components we derived the rms momentum width and horizontal emittance growth behavior. We also tried to understand the beam width oscillation after transition by extracting relevant parameters through a fitting model. We analyzed the post-transition horizontal beam size oscillation based on a model where the longitudinal phase space mismatch has resulted from non-adiabatic motion in the transition energy crossing. We also estimated the space charge effect during the transition energy crossing. A space charge stopband correction approach is proposed as a means to reduce the emittance growth due to space charge effect. 


\subsection{The Emittance Measurements}

IPM data for Booster (under event 17) were taken with various intensity levels on Feb. 3, 2005. The injected-turn numbers were varied from 2 to 18 . The gate of the ionization profile monitor is about $1 \mu \mathrm{s}$, or the profile is the average of about 52 bunches. The experimental conditions for all data sets were the same as the normal operations. The corrector package settings were recorded to build a realistic lattice model for lattice function calculations.

The profile data at each turn is fit with a Gaussian plus polynomial model [45],

$$
p(y)=a+b y+A \exp \left(-\frac{\left(y-y_{0}\right)^{2}}{2 \sigma_{y}^{2}}\right),
$$

where $y$ is the transverse coordinate of a micro-strip and $p$ is the reading of the strip. The parameter $\sigma_{y}$ and $y_{0}$ are the half rms width and central position of the beam, respectively. The calibration routine with linear parameterization is used to compensate the systematic error of beam width due to space charge [7]. The calibration makes significant corrections for high intensity and small beam width cases. Thus the calibration is much more important for the horizontal beam profile, because $\beta_{x} \approx 6.5 \mathrm{~m} \ll \beta_{z} \approx 20.5 \mathrm{~m}$ and the dispersion $D_{x}=1.8 \mathrm{~m}$ at the IPM location.

The rms beam width $\sigma_{y}$ in Eq. (6.1) is related to the emittance by

$$
\epsilon_{y, \mathrm{rms}}=\frac{\sigma_{y}^{2}}{\beta_{y}},
$$

where $\epsilon_{\mathrm{rms}}$ is rms emittance. The $95 \%$ emittance $\epsilon_{0.95}$ is $\epsilon_{0.95}=6 \epsilon_{\mathrm{rms}}$. These relations can be applied to vertical data directly. For horizontal data, the distribution of momentum deviation also contribute to the beam width. Assuming that the horizontal transverse phase-space distribution is un-correlated to the longitudinal phase-space distribution, we obtain

$$
\left\langle x^{2}\right\rangle=\left\langle x_{\beta}^{2}\right\rangle+D^{2}\left\langle\delta^{2}\right\rangle \quad \text { or } \quad \sigma_{x}^{2}=\beta_{x} \epsilon_{x, \mathrm{rms}}+D^{2} \sigma_{\delta}^{2}
$$


where $\langle\cdots\rangle$ denotes ensemble average, $\delta=\Delta p / p, D$ is the dispersion at the IPM and $x_{\beta}$ is the betatron part of the horizontal orbit, $\epsilon_{x, \mathrm{rms}}$ is the rms horizontal emittance, and $\sigma_{\delta}$ is the rms momentum width. The transverse emittances decrease as the beam momentum is ramped up due to adiabatic damping. The normalized emittance defined as $\epsilon_{\mathrm{n}}=\beta \gamma \epsilon$ is supposed to be conserved if non-conservative mechanisms are absent.
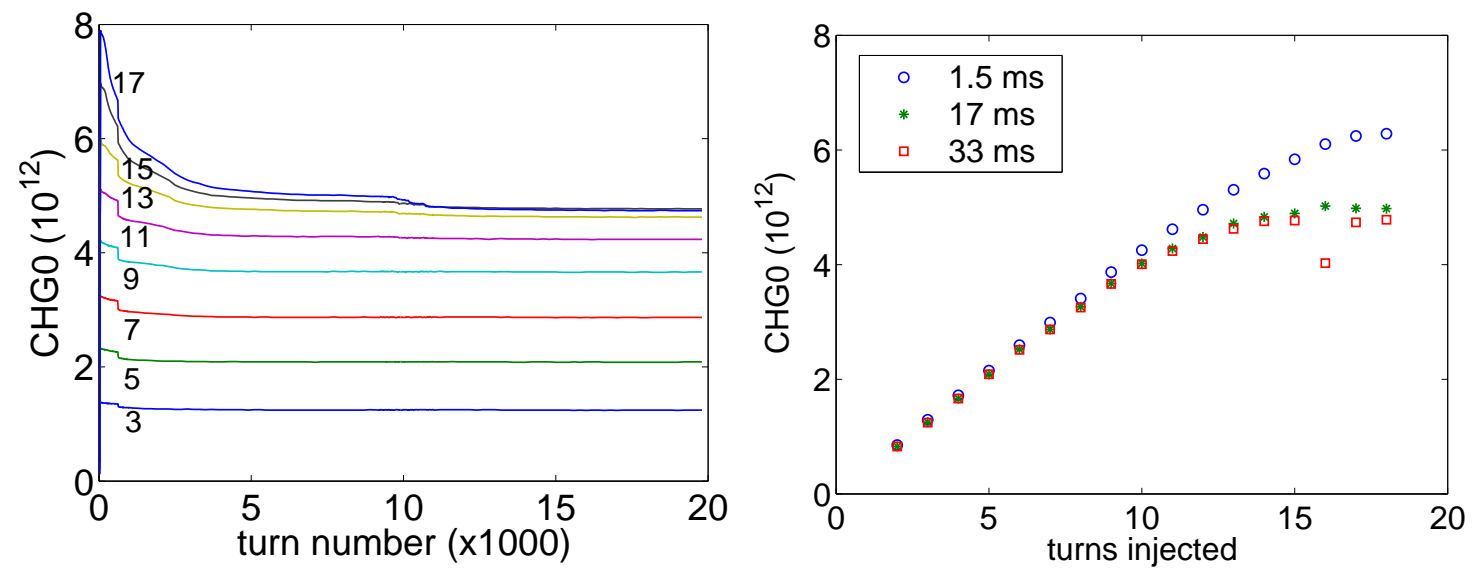

Figure 6.1: Left: total charge (CHG0, in $\left.10^{12}\right)$ for data sets with $3,5,7$, etc. injection-turns. Note that the notch of beam loss at about turn-600 is created intentionally by kicking 3 bunches out to facilitate the extraction to the Main Injector. Right: CHG0 after the notch (1.5 ms), right before transition $(17 \mathrm{~ms})$ and at extraction (33 ms) for all data sets. Note the 16-turn injection data set lost beam at transition accidentally.

The charge signals were taken along with IPM data. Fig. 6.1 shows the total charge for some data sets and the total charge at three characteristic points of the cycle for all 
data sets. Note that small loss occurs at high injection-turn after transition crossing. Beam-loss becomes very severe at injection turns larger than 12 .

The transverse space charge effect is characterized by the generalized space charge perveance, defined as

$$
K_{s c}=2 N r_{0} /\left(\beta^{2} \gamma^{3}\right),
$$

where $N$ is number of protons per unit length and $r_{0}=1.5347 \times 10^{-18} \mathrm{~m}$. Taking a Gaussian beam distribution with $N=N_{\mathrm{B}} /\left(\sqrt{2 \pi} \sigma_{s}\right)$, where $\sigma_{s}$ is the longitudinal rms bunch length, we plot the space charge perveance for a Booster cycle for 10-turn injection in Fig. 6.2.

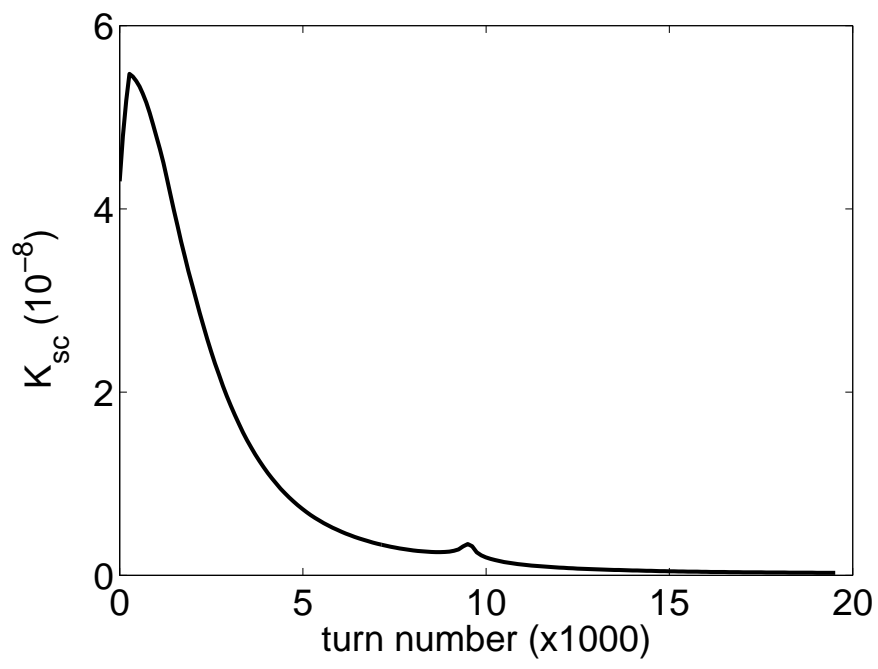

Figure 6.2: The space charge perveance parameter for an entire Booster ramping cycle for the beam intensity of 10-turn injection (total charge of 4.1E12). The bunch length is derived from the total rf voltage (RFSUM) of the Booster cycle while the $95 \%$ longitudinal emittance is assumed to be $0.08 \mathrm{eV}$-s. 
For emittance calculation in the entire cycle, we build the lattice model at $1 \mathrm{~ms}$ interval in the cycle according to the actual beam energy and experimental lattice setting. The lattice functions such as beta functions $\beta_{x}, \beta_{z}$, dispersion function $D$ and transition gamma $\gamma_{t}$ anywhere in the cycle are calculated with interpolation. Fig. 6.3 shows the change of these lattice functions in the cycle at IPM location.

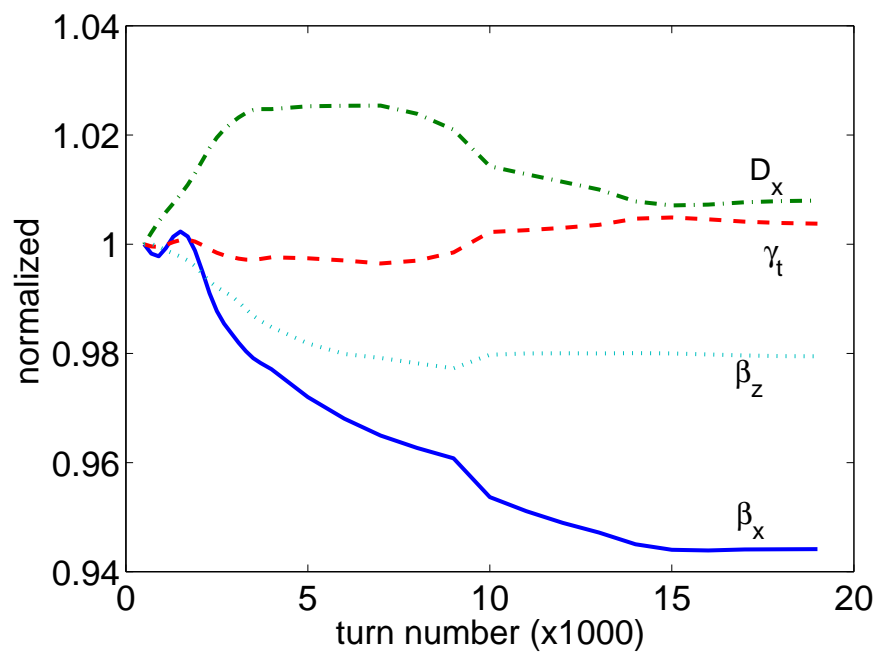

Figure 6.3: Lattice functions $\beta_{x}, \beta_{z}, D_{x}$ at the IPM location and the transition $\gamma_{t}$ normalized by the values at $1 \mathrm{~ms}$, where $\beta_{x 0}=6.5 \mathrm{~m}, \beta_{z 0}=20.5 \mathrm{~m}, D_{x 0}=1.8 \mathrm{~m}$ and $\gamma_{t 0}=5.48$. The change of these lattice functions arises from the effect of the DC dogleg magnets and the trim quadrupoles.

The turn-by-turn beam width signal from the IPM is usually noisy. Since we are interested only in the average behavior, it is useful to filter out the high-frequency noise. We apply a 40-points low-pass digital filter with tune threshold 0.1 to remove such noise. The effects of the filter are shown in Fig. 6.4 as an example for the 
vertical mean-square beam size in the vertical plane. Fig. 6.5 shows the properties of the digital filter.

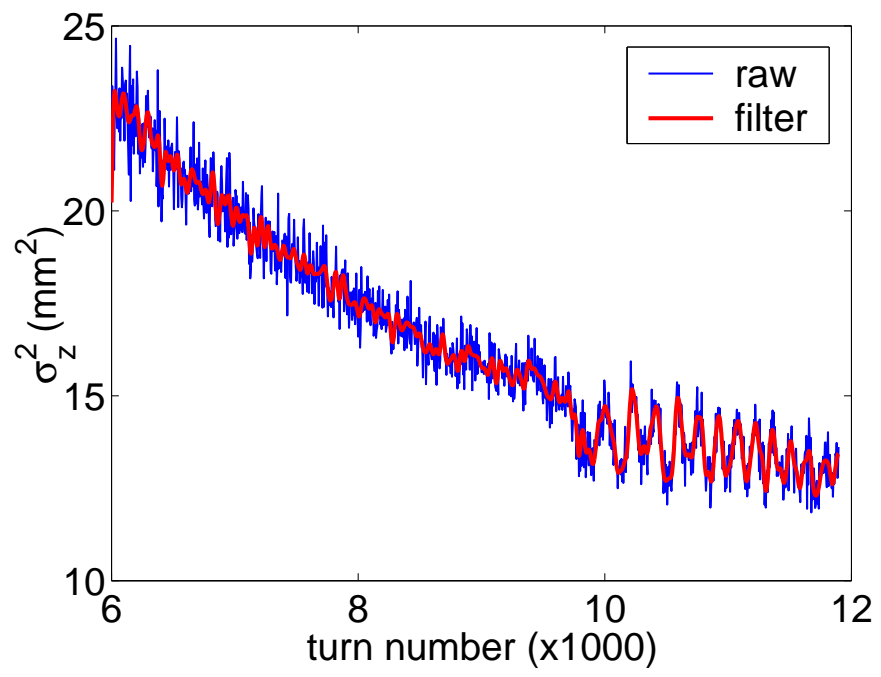

Figure 6.4: The raw and filtered $\sigma_{z}^{2}$ for 10-turn injection are shown for comparison.

\subsection{The Vertical Emittance}

Since the transverse emittances are adiabatically damped as the momentum increases, it is more convenient to study the normalized emittance. In this section we study the vertical emittance because it is free of the complication of longitudinal distribution. The normalized vertical emittance is calculated using the actual vertical beta function $\beta_{z}$ of the time of the cycle. Fig. 6.6 shows the vertical rms emittance for two data sets with 4 injection-turns or 12 injection-turns respectively. We note the emittance starts with the same level $(2 \pi$-mm-mrad) but follows different growth pattern for different intensities. 

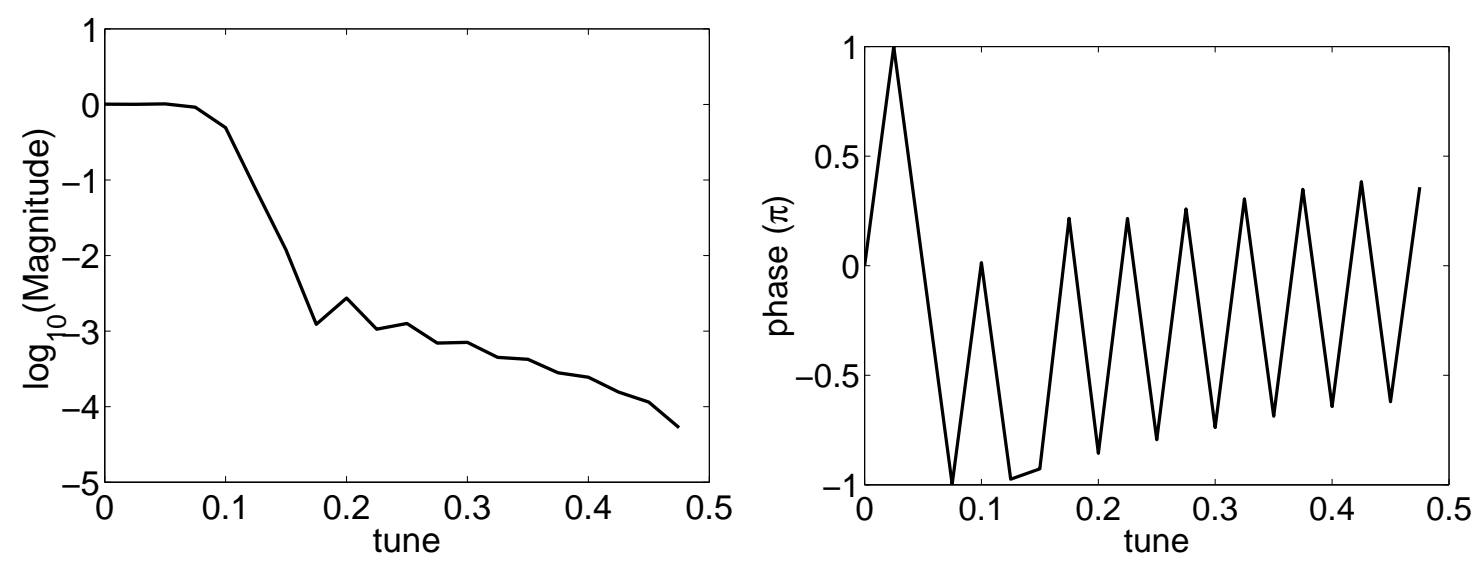

Figure 6.5: The magnitude (left plot) and phase (right plot) properties of the digital filter.

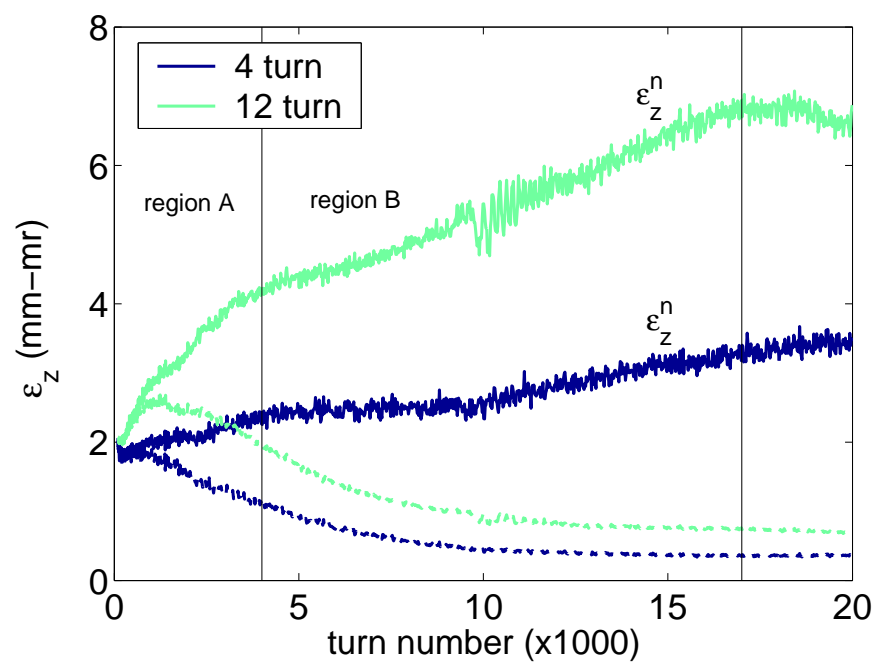

Figure 6.6: The vertical rms emittance (dashed) and the normalized rms emittance (solid) in the entire cycle for 4-turn and 12-turn injection. 
The normalized emittance grows rapidly in the first 4000 turns because of the space charge effect and the growth rate is nonlinear and highly dependent on beam intensities. For the later part of the cycle (4001-17000 turns), except close to extraction, the growth is slow and linear. The cause of the later emittance growth is not likely the space charge effect because the relativistic gamma is large in this region. Possible growth mechanisms are residual gas scattering, intra-beam scattering, or other mechanisms. We will treat the two growth periods separately with the first period (region A) contains from turn 70 after injection to turn 4000 and the second period (region B) contains from turn 4001 to turn 17000 .

\subsubsection{Linear growth region}

The emittance growth in region B can be roughly considered as linear. However some data-sets may lose beam during the transition energy crossing and some have posttransition oscillation. We fit the pre-transition and post-transition regions to linear curves separately. The fitting curves are shown in Fig. 6.7.

The growth rate of emittance defined by

$$
\alpha_{z}=\frac{\Delta \epsilon_{z}^{n}}{\Delta N}
$$

where $N$ is number of turns, is calculated with the slopes of the linear curves. The growth rates are shown in Fig. 6.8. The vertical normalized emittance grows about 1 $\pi$-mm-mrad in $10^{4}$ turns for normal working cycles of the Booster. The growth rate seems to be proportional to the injection-turns.

The growth rate due to residual gas scattering is estimated to be $\Delta \epsilon^{n} /\left(\epsilon^{n} \Delta t\right)=$

$0.008 \mathrm{~s}^{-1}$ at the vacuum pressure of $100 \mathrm{nTorr}$, which is about $1.2 \times 10^{-4}$ in $15 \mathrm{~ms}$ (or 10000 turns). Thus the emittance growth can not be caused by the residual gas small-angle scattering. The growth rate due to the intrabeam scattering is estimated 

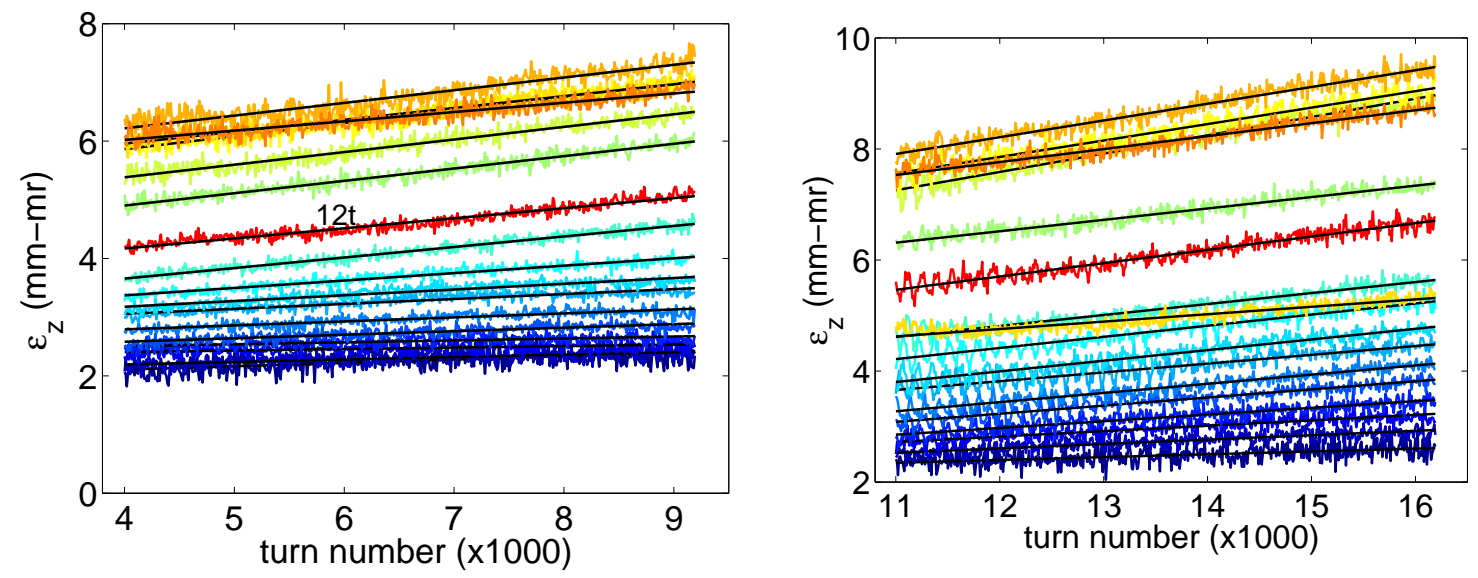

Figure 6.7: Left: (Color) The normalized vertical rms emittance from turn 4001 to turn 9200 for all data sets with 2turn injection to 18 turn injection. Right: (Color) The normalized vertical rms emittance from turn 11001 to turn 16200 for all data sets with 2-turn injection to 18turn injection. Note 16-turn data set lost a big fraction of beam at transition and thus has smaller emittance here. 


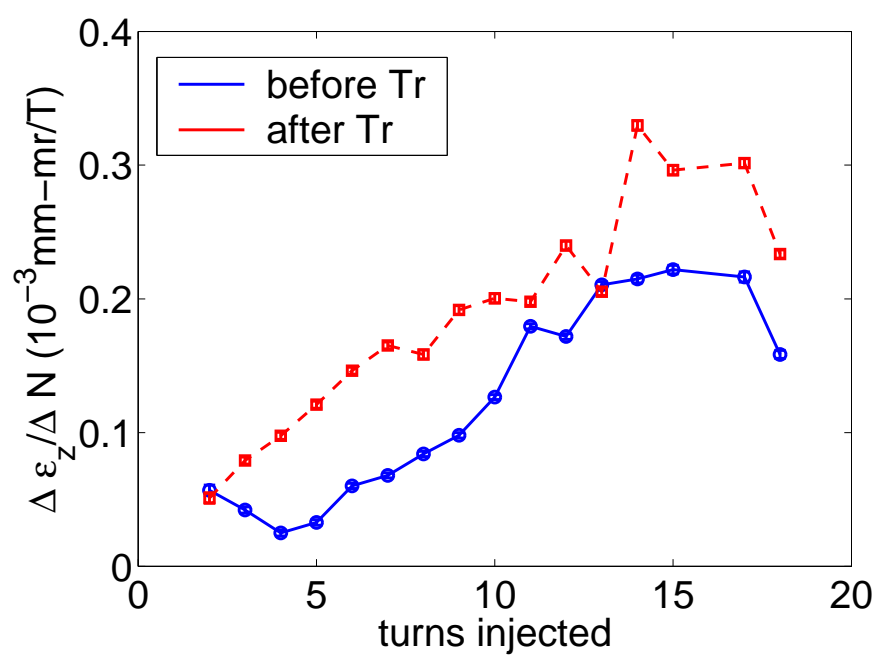

Figure 6.8: (Color) The vertical emittance growth rate before and after transition for all data sets.

to be less than $\Delta \epsilon^{n} / \epsilon^{n} \Delta t \leq 0.001 \mathrm{~s}^{-1}[47]$. It is difficult to see why the growth rate of the emittance is as large as $1 \pi$-mm-mrad in $10^{4}$ turns.

\subsubsection{Space charge dominated region}

Space charge effects play an important role in the first 4000 turns. Fig. 6.9 shows the normalized vertical rms emittance in region A for all data sets. Note that with 12 injection-turns or less, the emittance growth is relatively mild. The growth behavior for these data sets follows a similar pattern, which could be modeled. However, for 14 turns or more injection, the emittances grew violently in the first few hundreds of turns and ended up with much higher values at turn 4000 than the other cases with less intensity. The 13-turn injection data set is like an intermediate behavior. It follows the pattern of the first kind at the beginning but starts blowing up from about turn 1500 . 


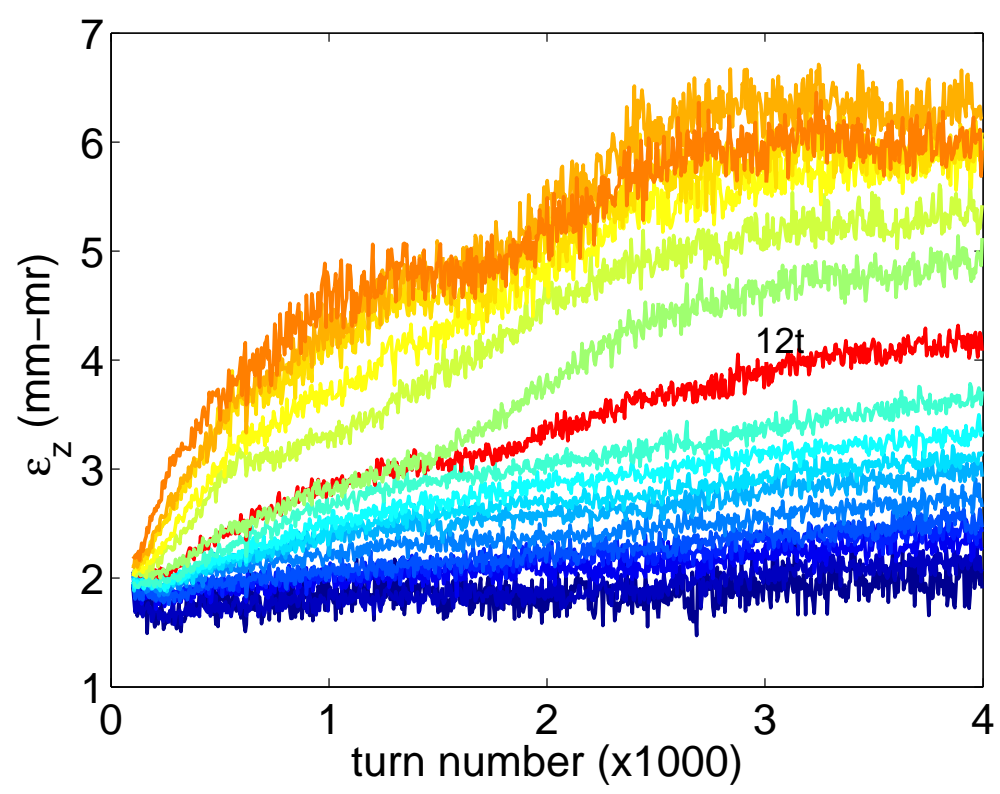

Figure 6.9: (Color) The normalized vertical rms emittance from turn 70 to turn 4000 for all data sets with 2-turn injection to 18-turn injection. Note the RED curve is for 12-turn injection which marks the border of two kinds of emittance growth behavior. 
Emittance dilution due to space charge is well-known. However, the exact emittance growth mechanism is unclear. Thus the high space charge cases are certainly much more difficult to analyze. A popular belief is that the emittance dilution has resulted from the half-integer stopband of the betatron motion. The stopband width is proportional to the space charge perveance $K_{s c}$. It is tantalizing to find a relation between the space charge parameter and the growth rate for the low space charge end.

Let the instantaneous growth rate be proportional to $K_{s c}$ :

$$
\frac{d \epsilon}{d t}=b_{1}+b_{2} K_{s c}
$$

The normalized emittance can then be expressed as

$$
\epsilon=a_{0}+b_{1} t+b_{2} \int_{0}^{t} K_{s c} d t^{\prime}
$$

where $a_{0}$ denotes initial normalized emittance, $b_{1}$ denotes linear growth rate and $b_{2}$ denotes space charge induced growth rate. We can fit $\epsilon_{z}^{n}$ to this model. Some fitting curves are shown in Fig. 6.10. The fitting parameters are shown in Fig. 6.11. The fitted linear growth rate is the same as the before-transition curve shown in Fig. 6.8. The space charge dependent growth rate $b_{2}$ is nearly constant for all data sets because the intensity has been absorbed by the perveance parameter $K_{s c}$.

An alternative model is based on Eq. (6.16) of Ref. [48], which leads to,

$$
\left(\epsilon_{x}^{n}\right)^{2}=a_{0}\left(1+b_{1} t+b_{2} \int_{0}^{t} K_{s c} d t^{\prime}\right) .
$$

This model describes emittance growth due to initial non-equilibrium distribution of the beam. The fitting result has very similar features as the model of Eq. (6.6). 

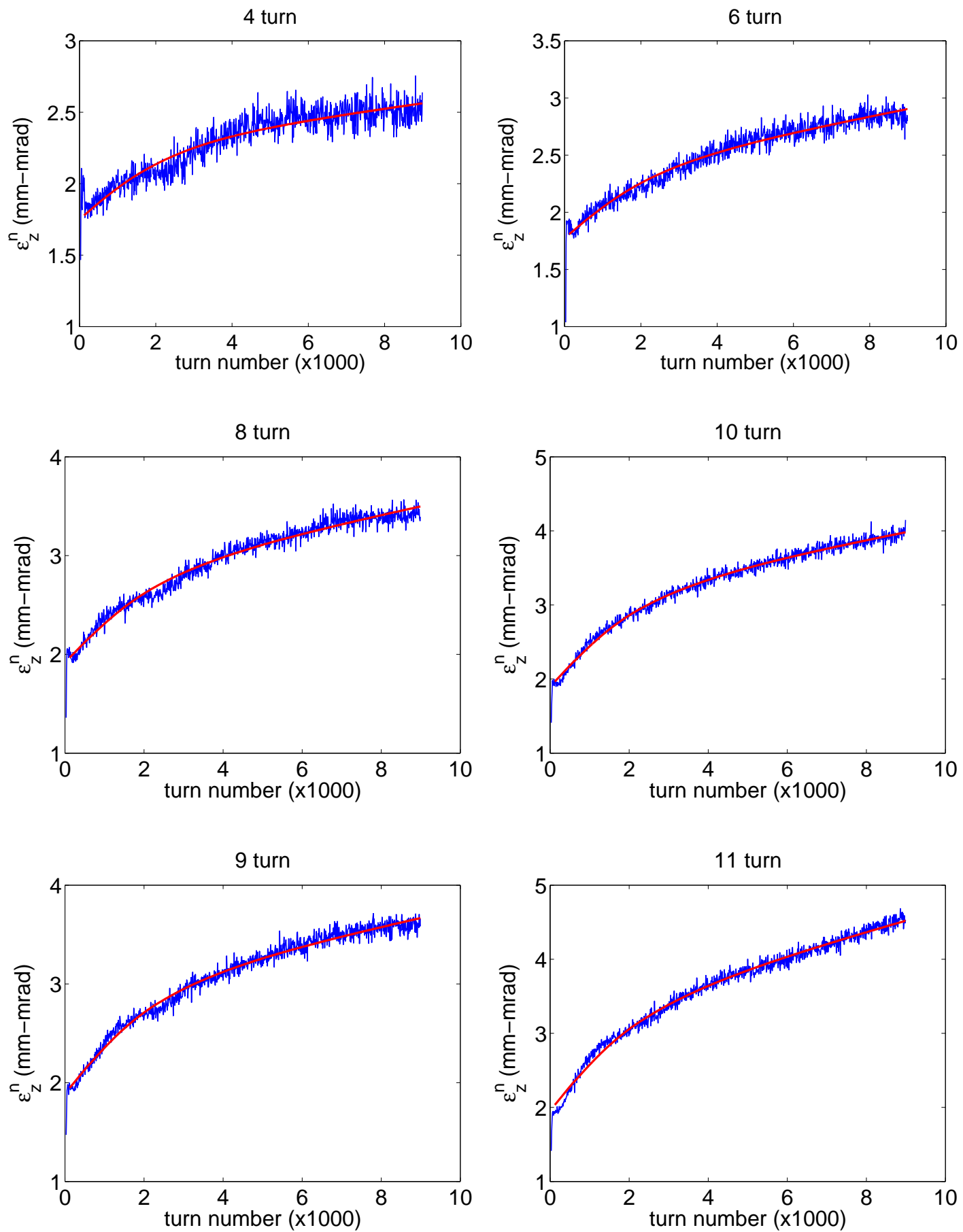

Figure 6.10: The fitting curves of model Eq. (6.6) for some data sets with various turns injected. 

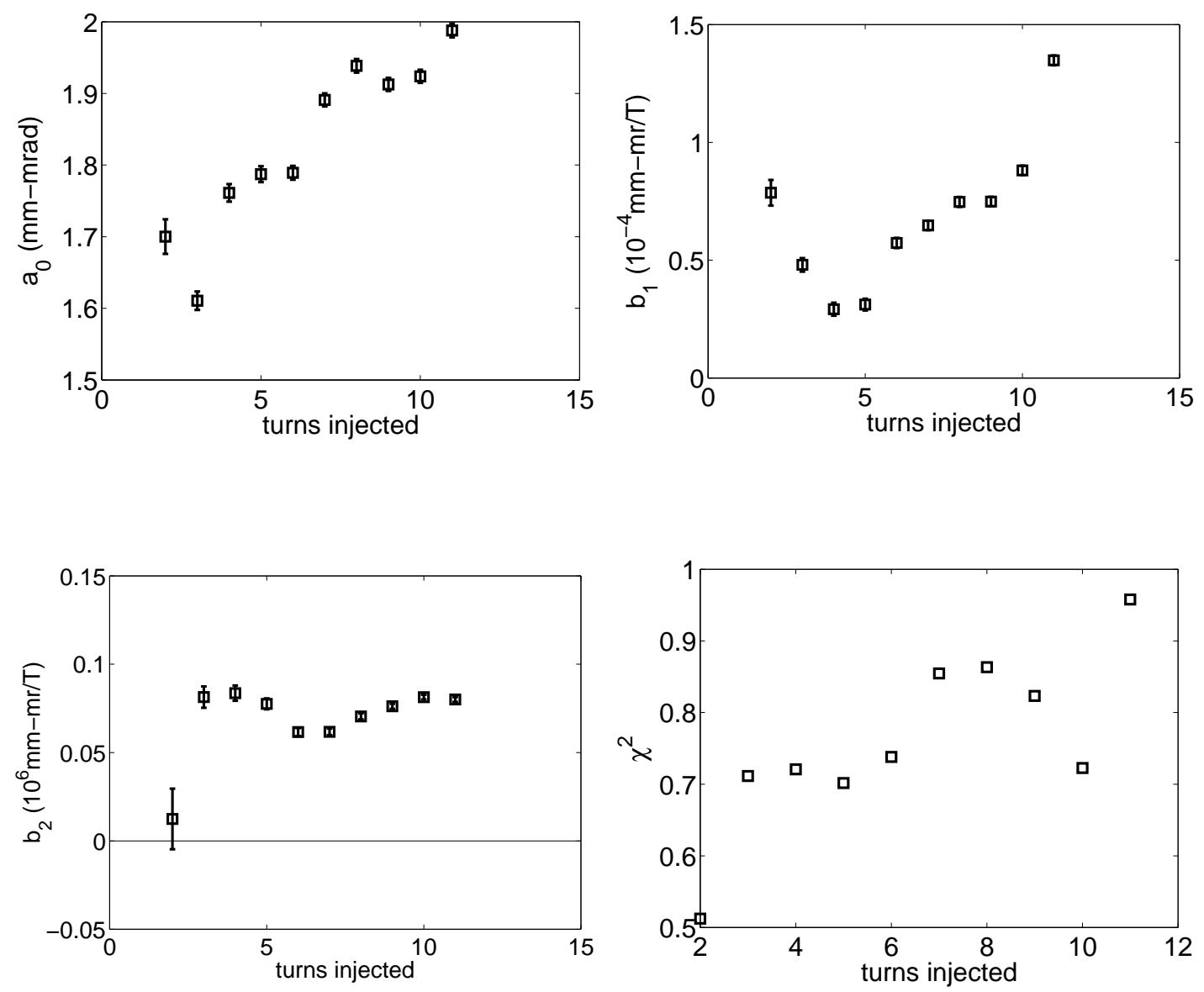

Figure 6.11: Top left: parameter $a_{0}$, initial vertical normalized emittance. Top right: parameter $b_{1}$, linear growth rate. Bottom left: parameter $b_{2}$, space charge dependent growth rate. Bottom right: the residual $\chi^{2}$, normalized by noise sigma and number of data points. 


\subsection{Horizontal Emittance}

The horizontal beam width reflects both the horizontal emittance and the longitudinal off-momentum distribution as seen in Eq. (6.3). Fig. 6.12 shows $\sigma_{x}^{2}$ for 4-turn and 12-turn injection for the entire ramping cycle. The off-momentum width is increased around transition because the bunch length is shortened. The beam width starts oscillating after transition because of the longitudinal phase space mismatch.

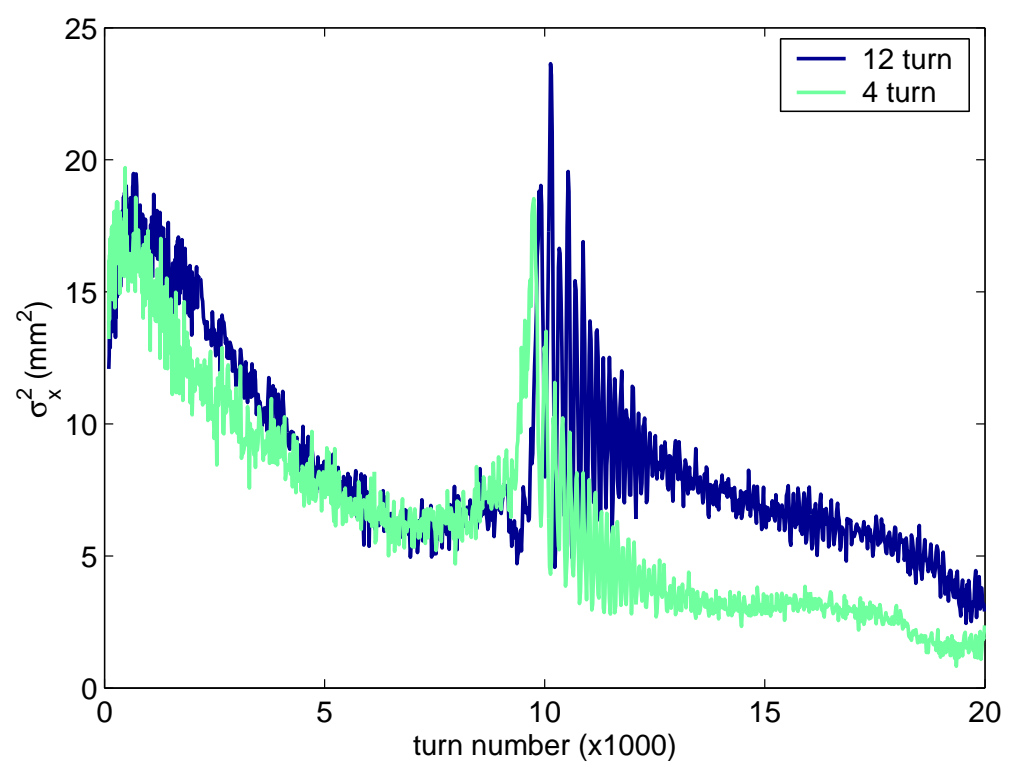

Figure 6.12: (Color) $\sigma_{x}^{2}$ for 4-turn and 12-turn injection. The beam widths below and above transition energy shows adiabatic damping, while the beam width is enhanced at the transition energy region due to the increase of the momentum width.

Since the transverse horizontal emittance and the momentum width have different scaling property as a function of the beam momentum, we cannot calculate the normalized horizontal emittance directly unless we can isolate and remove the contri- 
butions of the off-momentum distribution. In the following, we use the fact that the energy scaling rules of the betatron emittance and the off-momentum variables are different to separate the contribution of the betatron and off-momentum components.

\subsubsection{Below transition energy}

One way to obtain the transverse betatron component in $\sigma_{x}^{2}$ is to subtract the "known"

off-momentum width component, i.e. $\sigma_{x, \beta}^{2}=\sigma_{x}^{2}-D^{2} \sigma_{\delta}^{2}$. We can do so by assuming the beam fills up the rf bucket during the adiabatic capture at injection. The bunch shape then follows the evolution of the rf bucket as a matched beam, which can be determined knowing the rf voltage $V$ and $\mathrm{rf}$ synchronous phase $\phi_{s}$. Fig. 6.13 shows the rf voltage (RFSUM), recorded with console program during the experiment, and the rf synchronous phase, calculated by the energy gain per turn according to the magnetic field ramp. Fig. 6.14 shows the rf bucket area (left plot), the rms momentum width and bunch length (right plot) calculated by assuming that the phase space area of a bunch to be $0.08 \mathrm{eV}$-s. The longitudinal component in $\sigma_{x}^{2}$ can then be calculated with $D^{2} \sigma_{\delta}^{2}$.

Another way is to make use of the difference of the scaling rules of the transverse and longitudinal components with respect to the rf voltate and the beam momentum. Both terms of Eq. (6.3) change in the cycle as the momentum is ramped up. However, they change in different scaling rules. The scaling rules are shown in Eq. (6.8)

$$
\sigma_{x}^{2}=\beta_{x} \epsilon_{\mathrm{rms}}+D^{2} \sigma_{\delta}^{2}=\beta_{x} \frac{\epsilon_{\mathrm{rms}}^{n}}{\beta \gamma} \frac{\beta_{x 0}(\beta \gamma)_{0}}{\beta_{x 0}(\beta \gamma)_{0}}+D^{2} \sigma_{\delta 0}^{2} \frac{\sigma_{\delta}^{2}}{\sigma_{\delta 0}^{2}}=a A(t)+b B(t)
$$

with

$$
\begin{aligned}
& a=\epsilon_{\mathrm{rms}}^{n} \frac{\beta_{x 0}}{\beta_{0} \gamma_{0}}, \quad A(t)=\frac{\beta_{x}}{\beta_{x 0}} \frac{\beta_{0} \gamma_{0}}{\beta \gamma} \\
& b=D^{2} \sigma_{\delta 0}^{2}, \quad B(t)=\frac{\gamma_{0} \sqrt{\gamma_{0}\left|\eta_{0}\right| / V_{0}\left|\cos \phi_{s 0}\right|}}{\gamma \sqrt{\gamma|\eta| / V\left|\cos \phi_{s}\right|}}
\end{aligned}
$$




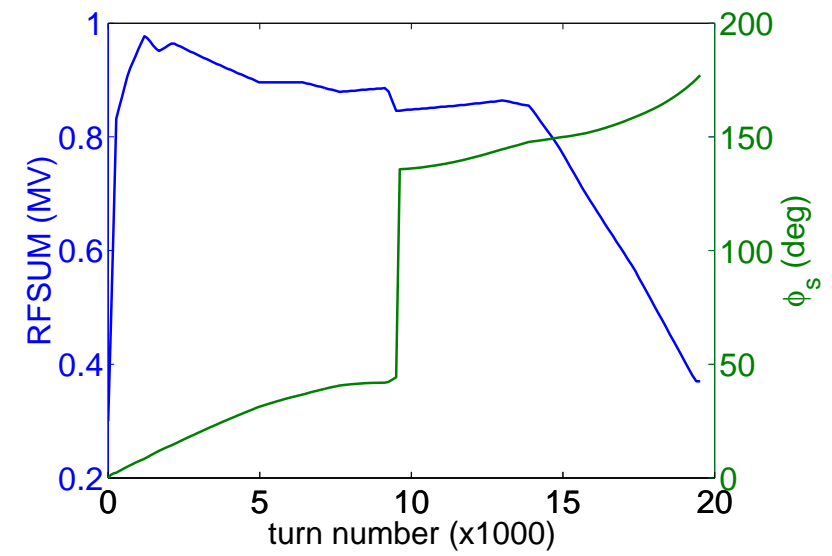

Figure 6.13: (Color) The rf voltage $V$ (RFSUM) and the synchronous phase $\phi_{s}$.
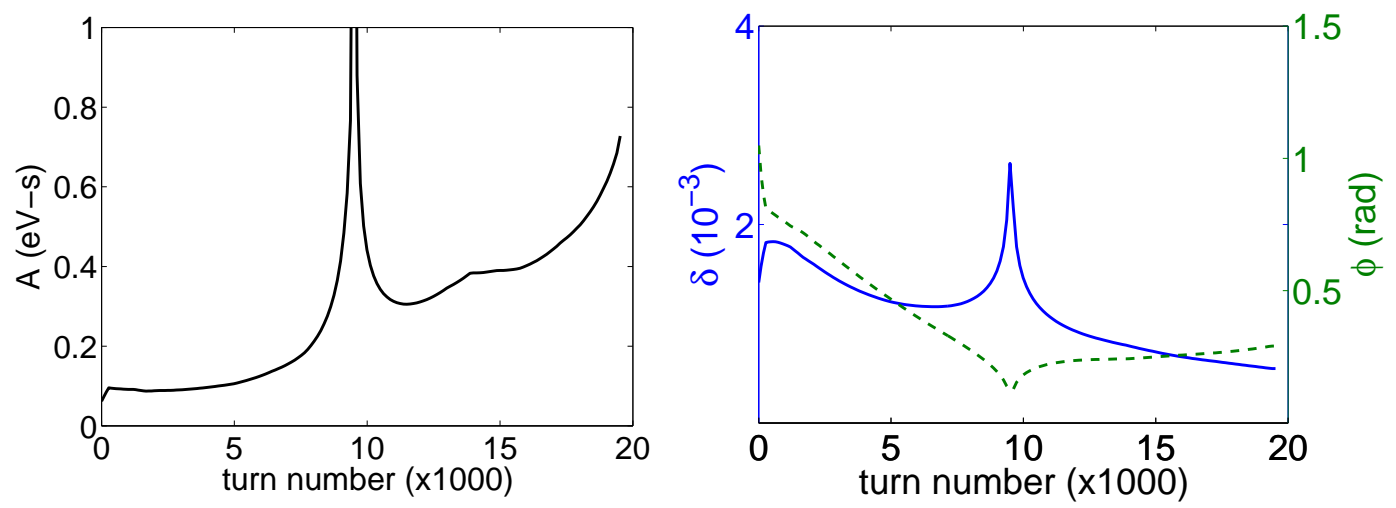

Figure 6.14: Left: The bucket area throughout the Booster cycle. The bucket area is about $0.08 \mathrm{eV}$-s at the beginning of the ramping cycle. Right: (Color) The rms momentum width $\sigma_{\delta}$ and rms bunch length $\sigma_{\phi}$, assuming phase space area $A=0.08 \mathrm{eV}$-s. The rms value is obtained by dividing the maximum $\delta$ and $\phi$ by $\sqrt{6}$. 
where we have used the scaling rule of $\sigma_{\delta} \sim V^{1 / 4}|\eta|^{-1 / 4} \gamma^{-3 / 4}[1]$. The scaling rules of $A(t)$ and $B(t)$ are shown in Fig. 6.15.

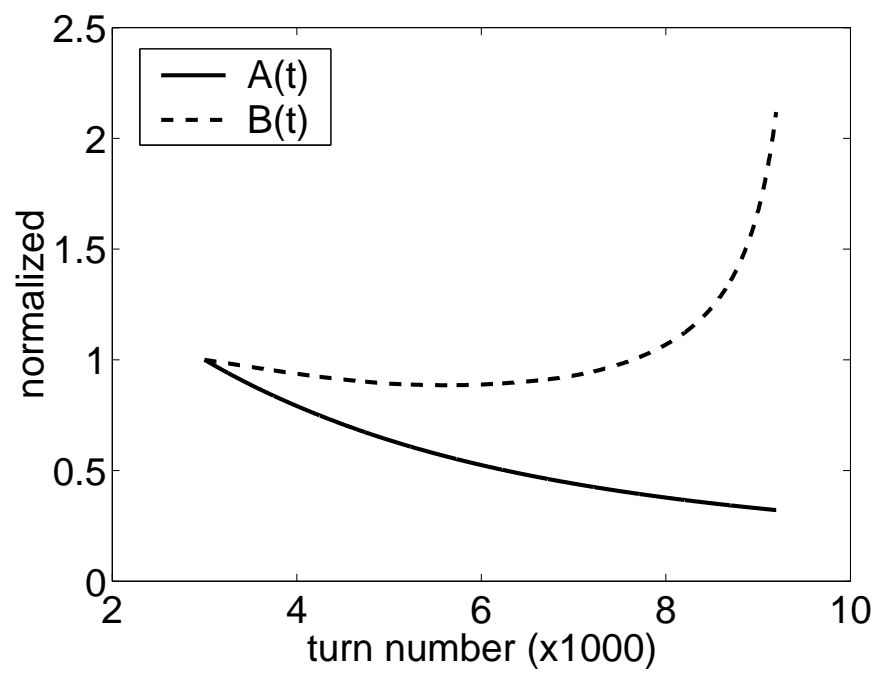

Figure 6.15: Scaling function $A(t)$ and $B(t)$, obtained with realistic Booster lattice model and rf parameters.

The normalized horizontal emittance can be considered as growing linearly if it has the same behavior as the vertical emittance, so we further assume $a=a_{0}+a_{1} t$. We assume $b=b_{0}$ is a constant, i.e. the longitudinal phase space area is preserved. We can fit the horizontal width with

$$
\sigma_{x}^{2}=\left(a_{0}+a_{1} t\right) A(t)+b_{0} B(t)
$$

To avoid the nonlinear emittance blow-up in the first several milliseconds of the cycle and the non-adiabatic region near transition, we fit $\sigma_{x}^{2}$ from turn 3001 to turn 9200 to the model of Eq. (6.11) to obtain constant parameters $a_{0}, a_{1}$ and $b_{0}$ for each data set and turn these parameters to horizontal emittance or rms momentum width according to Eqs. (6.9), (6.10). Some fitting curves are shown in Fig. 6.16.

This model does not describe the data of high-intensity cycles very well because 

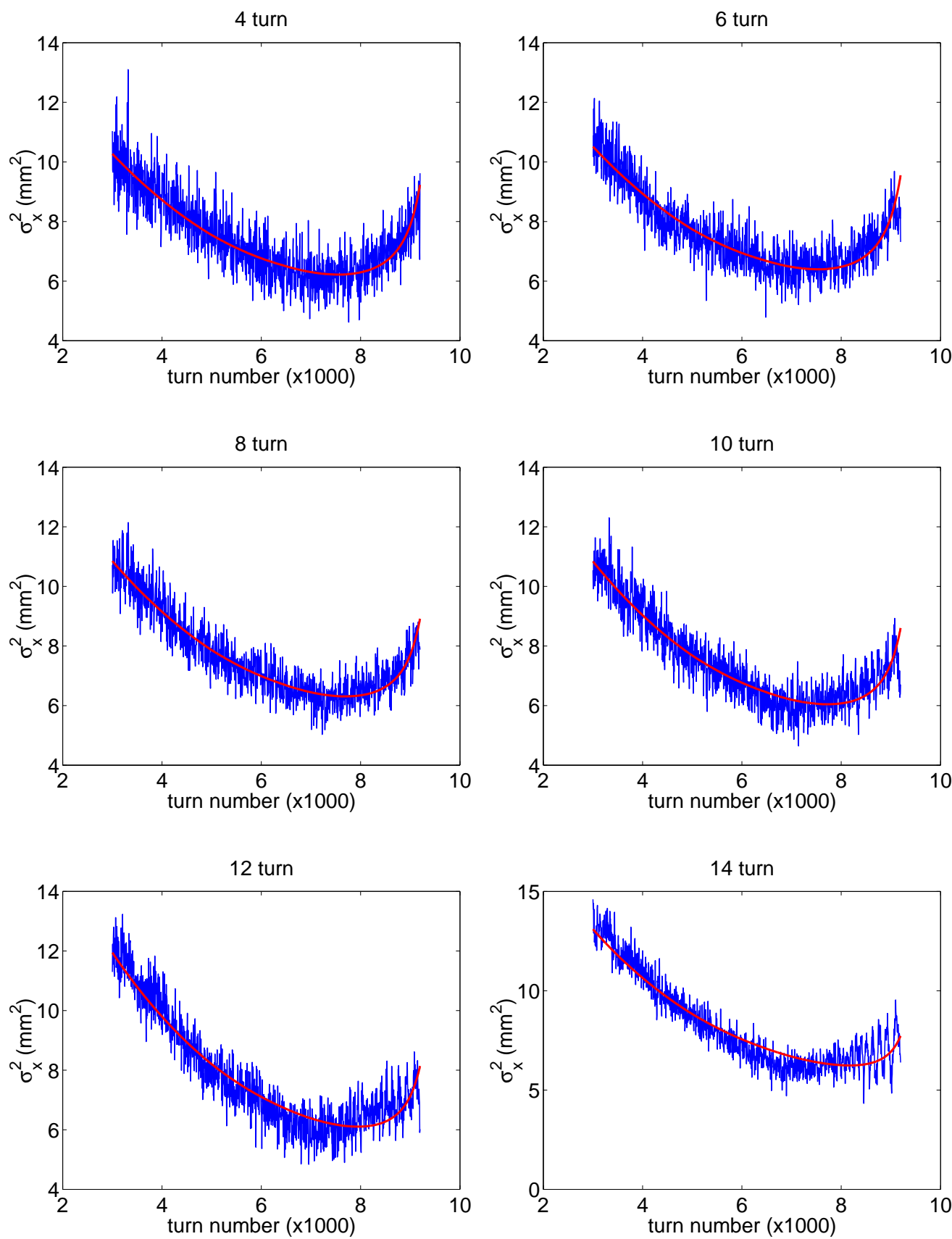

Figure 6.16: The fitting curves of Eq. (6.11) for some data sets with various turns injected. 
the calibration result of the linear parameterization scheme deviates from the actual beam size for high intensity beams (See Fig. 2 of Ref. [7]). The horizontal beam size $\sigma_{x}$ (see Fig. 6.12) drops from $3.5 \mathrm{~mm}$ (at turn 3001 ) to $2.5 \mathrm{~mm}$ (near turn 7000 ) in this region. For a beam of $\sigma_{x}=3 \mathrm{~mm}$ with total charge of $4 \mathrm{E} 12$ (i.e., 10-turn injection), the calibration error is as large as 5\%. As the beam size shrinks due to adiabatic damping, the deviation gets even more severe. Such deviation destroys the scaling laws in the calibrated data and leads to unreasonable fitting results. Hence we show the results only for data sets with 10-turn injection or less. The resulting normalized emittance, its growth rate and the rms momentum width are shown in Fig. 6.17. The standard deviations of the noises in $\sigma_{x}^{2}$, along with the covariance matrix of the fitting, are used to estimate the error bars of these parameters.

The results give reasonable values of emittances and rms momentum widths at turn 3001 . The horizontal normalized emittances are found to be about $2 \pi$-mmmrad. This value is about the same as that of the initial vertical emittance (see $a_{0}$ of Fig. 6.11). The growth rate, defined as

$$
\alpha_{x}=\frac{\Delta \epsilon_{x}^{n}}{\Delta N}
$$

is about $0.8 \pi$-mm-mrad per $10^{4}$-revolutions. The horizontal growth rate is on the same level as the vertical growth rate shown in Fig. 6.8. The rms momentum width is about $1.0 \times 10^{-3}$, which is smaller than the value $1.4 \times 10^{-3}$ as predicted in Fig. 6.14. The momentum width for these data sets were nearly equal, indicating that particles almost fill up the rf buckets at injection.

We have measured the momentum spread using the resistive wall monitor signal with a high resolution scope for 4-turn and 11-turn injection. The recorded peaks on the beam current signal are fitted to elliptic model [46] to derive the bunch lengths. The bunch lengths are turned to momentum spread according to the phase space ellipses. The results are shown in Fig. 6.18. The rms momentum spread at turn 3001 

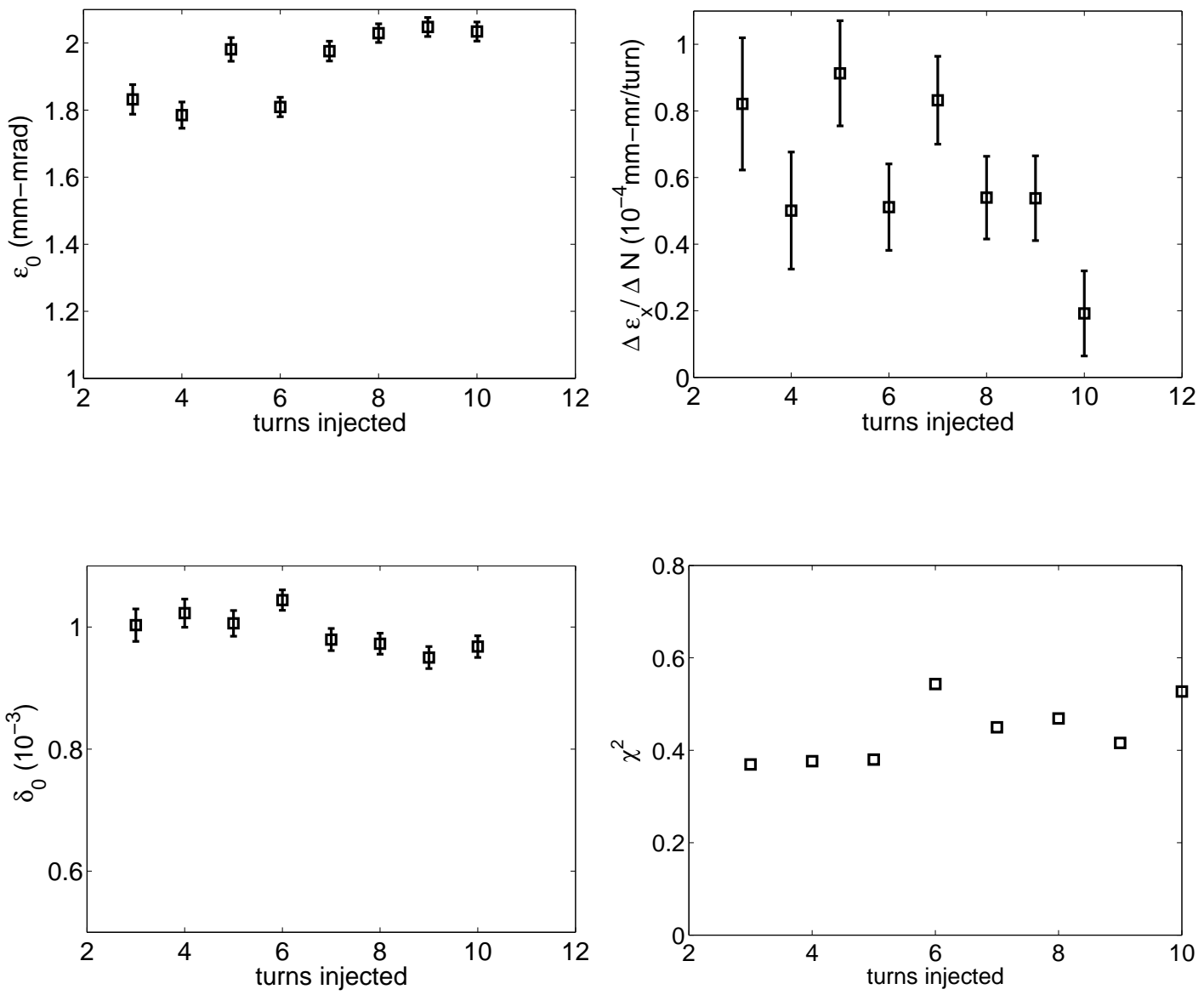

Figure 6.17: Top left: the fitted normalized horizontal emittance at turn $3001 \epsilon_{0}=a_{0} \frac{\beta_{0} \gamma_{0}}{\beta_{x 0}}$. Top right: the horizontal emittance growth rate defined as $\alpha_{x}=\frac{\Delta \epsilon_{x}^{n}}{\Delta N}$. Bottom left: the fitted rms momentum width $\sigma_{\delta}=\sqrt{b_{0}} / D_{0}$ at turn 3001 . Bottom right: the normalized residual $\chi^{2}$ for the fitting cases. 
is about $1.25 \times 10^{-3}$.
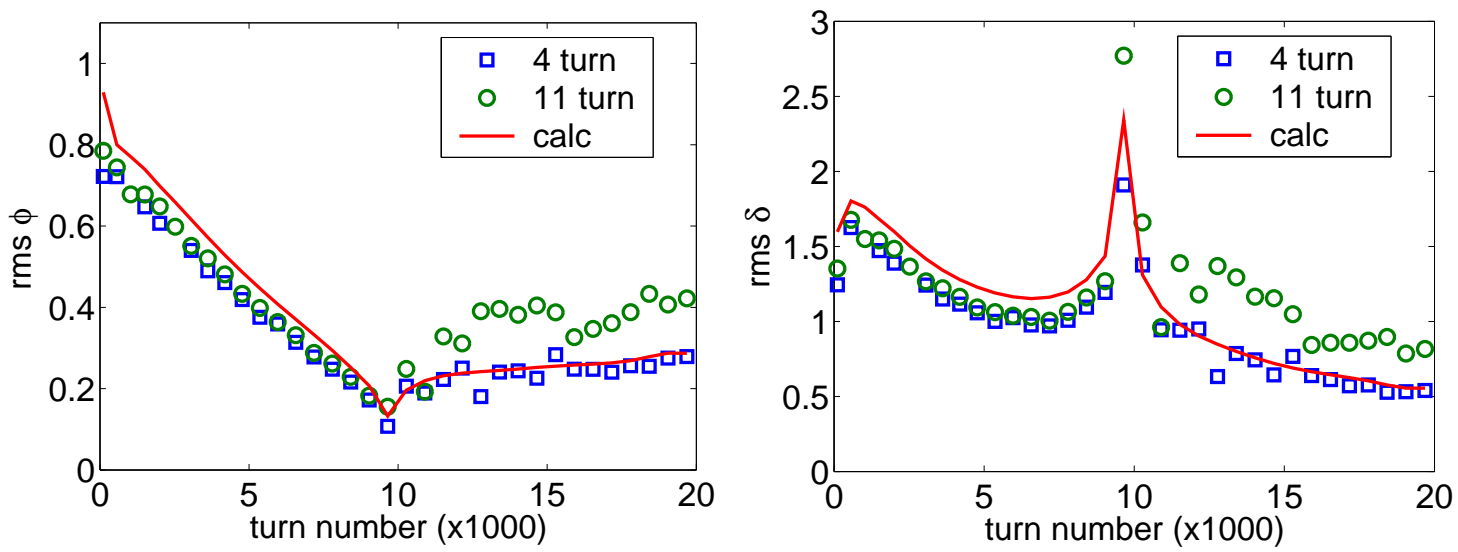

Figure 6.18: (Color) The measured rms bunch length $\sigma_{\phi}$ (left) and rms momentum width $\sigma_{\delta}$ (right) for 4-turn and 11turn injection are compared to calculations assuming phase space area $0.08 \mathrm{eV}$-s. The measurements were taken on April 29, 2005 under event 14.

\subsubsection{Across the transition energy}

The rms momentum width starts to grow rapidly as the beam gets near transition so that $\eta$ becomes small. The bunch shape cannot follow the rf bucket when it is very close to transition and the longitudinal motion is non-adiabatic. The adiabatic time and nonlinear time for Booster are estimated to be [1]

$$
\begin{aligned}
\tau_{\mathrm{ad}} & =\left(\frac{\pi \beta^{2} m c^{2} \gamma_{\mathrm{T}}^{4}}{\dot{\gamma} \omega_{0}^{2} h e V\left|\cos \phi_{\mathrm{s}}\right|}\right)^{1 / 3} \approx 0.20 \mathrm{~ms}, \\
\tau_{\mathrm{nl}} & =\gamma_{\mathrm{T}}^{3} \frac{\eta_{1}}{2 \dot{\gamma}} \hat{\delta}=\gamma_{\mathrm{T}} \frac{\frac{3}{2} \beta_{0}^{2}+\gamma_{\mathrm{T}}^{2} \alpha_{1}}{2 \dot{\gamma}} \hat{\delta} \approx 0.07 \mathrm{~ms},
\end{aligned}
$$


where we use $\dot{\gamma} \approx 392 \mathrm{~s}^{-1}, \alpha_{1}=1.0$, and the maximum rms momentum width at transition is obtained by using the non-adiabatic formula

$$
\left.\hat{\delta}\right|_{\gamma=\gamma_{\mathrm{T}}}=\frac{\gamma_{\mathrm{T}}}{3^{1 / 6} \beta \tau_{\mathrm{ad}} \Gamma\left(\frac{2}{3}\right)}\left(\frac{2 \mathcal{A}}{3 m c^{2} \dot{\gamma}}\right)^{1 / 2} \approx 0.502 \frac{\gamma_{\mathrm{T}}}{\beta \tau_{\mathrm{ad}}}\left(\frac{\mathcal{A}}{m c^{2} \dot{\gamma}}\right)^{1 / 2} .
$$

Here $\mathcal{A}$ is the rms phase space area of the beam in eV-s. The total growth due to nonlinear longitudinal motion is $G \approx \exp \left\{\frac{2}{3}\left(\tau_{\text {nl }} / \tau_{\text {ad }}\right)^{3 / 2}\right\} \approx 1.14$ [1]. Thus the phase space growth due to the nonlinear motion is about $14 \%$, which is small.

\subsubsection{Microwave instability}

The beam near transition energy can also suffer microwave instability. The emittance growth factor can be estimated as $G=\exp (S)$, where [49]

$$
S \approx \frac{n \omega_{0} N_{\mathrm{B}}^{2} e^{2}\left|Z_{\|} / n\right|^{2} \gamma_{\mathrm{T}}^{3}}{64 \pi^{2} \sigma_{\delta} \mathcal{A}^{2} \dot{\gamma}}
$$

where $\mathcal{A}=\pi \sigma_{E} \sigma_{t}$ is the rms phase space area in (eV-s), and $n=R / b \approx 1500$ is the mode number. Assuming a broadband impedance of $\left|Z_{\|}\right| / n \approx 20 \Omega$ and $N_{\mathrm{B}}=6 \times 10^{10}$, we find $G \approx 1.39$.

\subsubsection{Bunch mismatch oscillations in the synchrotron phase space}

After passing the non-adiabatic region near transition, the particles in the beam bunches start to follow the ellipses in the phase space again. However, the phase space shape of the bunches are mismatched to the ellipses. Hence the bunch starts to tumble in the bucket at the rate of synchrotron tune, which causes the rms momentum width of the beam to oscillate at the rate twice of the synchrotron tune. 
Let $\delta_{1}, \delta_{2}$ be the maximum and minimum rms momentum width, which are connected by the matched ellipses [1]

$$
\delta_{2}=\frac{\nu_{s}}{h \eta} \frac{\tilde{\mathcal{A}}}{\delta_{1}}
$$

where $\tilde{\mathcal{A}}=\pi \delta_{1} \phi_{1}$ is the rms phase space area. The extrema of horizontal beam width are related by

$$
\sigma_{x, \max }^{2}-\sigma_{x, \min }^{2}=D^{2}\left(\delta_{1}^{2}-\delta_{2}^{2}\right)
$$

We can identify $\hat{\delta}$ in Eq. (6.13) as $\delta_{1}$. The phase space area $\mathcal{A}$ (in $\mathrm{eV}$-s) in Eq. (6.13) is related with the phase space area $\tilde{\mathcal{A}}$ of Eq. (6.15) by

$$
\tilde{\mathcal{A}}=\frac{\omega_{0}}{\beta^{2} E} \mathcal{A}
$$

Combining Eqs. (6.13), (6.15) and (6.16), we can solve for $\delta_{1}, \delta_{2}$ and the phase space area $\mathcal{A}$ from the oscillation magnitude of $\sigma_{x}^{2}$.

The post-transition beam width oscillations may also arise from the mis-match across transition due to longitudinal space charge effect as pointed out by Sorensen [50]. In a linearized approximation, the longitudinal Hamiltonian around the transition energy region is

$$
H(\phi, \delta) \approx \frac{h \eta \omega_{0}}{2} \delta^{2}-\frac{\omega_{0} e}{4 \pi \beta^{2} E}\left[V \cos \phi_{\mathrm{s}}-\frac{h e c g_{0} Z_{0} N_{\mathrm{B}}}{2 \gamma^{2} R \sigma_{\phi}^{3}}\right]\left(\phi-\phi_{s}\right)^{2},
$$

where $h$ is the harmonic number, $g_{0}=1+2 \ln \frac{b}{a}$ is the geometric factor, $Z_{0}$ is the impedance of vacuum, $N_{\mathrm{B}}$ is the number of particles per bunch, and $R$ is the meanradius of the synchrotron. Note that the space charge force has de-focusing effect below transition energy $\left(\cos \phi_{s} \geq 0\right)$ and focusing effect above the transition energy $\left(\cos \phi_{s} \leq 0\right)$. It causes sudden change of the shape of matched ellipse and thus the mismatch between the beam bunch and the ellipse. The space charge effect can be 
estimated by evaluating the effective voltage of space charge relative to the $\mathrm{rf}$ focusing force $V \cos \phi_{s}$, or

$$
\frac{h e c g_{0} Z_{0} N_{\mathrm{B}}}{2 \gamma^{2} R \sigma_{\phi}^{3}} \frac{1}{V \cos \phi_{\mathrm{s}}}
$$

This parameter is shown in Fig. 6.19 for 10 injection-turns with a phase space area of $0.08 \mathrm{eV}$-s. The result shows that the maximum space charge voltage (at transition) is about $10 \mathrm{kV}$, or only $1.7 \%$ of the rf voltage at the time.

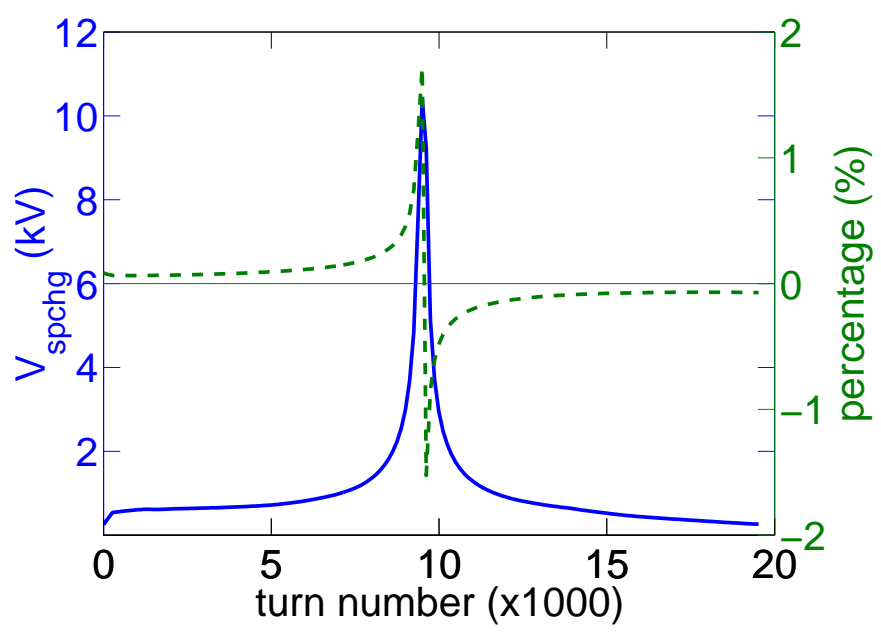

Figure 6.19: The effective space charge voltage $V_{\text {spchg }}($ solid) and the ratio of $V_{\text {spchg }} /\left(V_{r f} \cos \phi_{s}\right)$ (dashed).

It is commonly believed that the post-transition bunch length oscillation is due to the space charge potential which causes rf potential well distortion and induces bunch length mis-match. However, the post-transition data in the next section shows that the mismatch factor is about $\delta_{1} / \delta_{2} \approx 4.8$, which is nearly independent of the injection-turn. Thus the bunch shape mismatch due to the non-adiabatic motion during the transition crossing is the main cause of bunch length oscillation above the transition energy. This agrees with our space charge voltage estimation in Fig. 6.19. 


\subsubsection{Above transition energy}

The horizontal beam width oscillation can be seen in Fig. 6.12. After examining the oscillation pattern of $\sigma_{x}^{2}$ we found it can be fitted with a model

$$
\sigma_{x}^{2}(t)=a+b t+c t^{2}+A \exp (-\alpha t) \cos \left[2 \pi\left(f_{1} t+f_{2} t^{2}\right)+\chi\right]
$$

where $t$ is turn number. We apply this fitting model to data from turn 10501 to turn 13500 (transition is at turn 9500, but we avoid the jerk in the first 1000 turns). Examples of fitting curves are shown in Fig. 6.20. Fig. 6.21 and 6.22 show the fitting
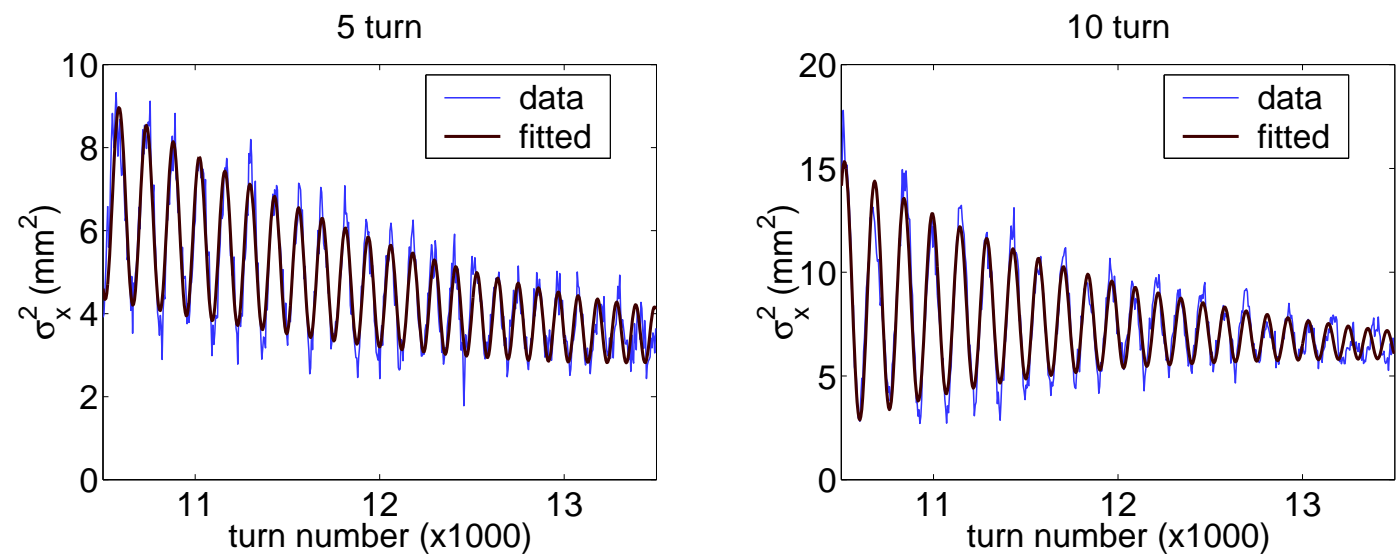

Figure 6.20: Left: Fit $\sigma_{x}^{2}$ to the model of Eq. (6.20) for 5 turns injection. Right: Fit $\sigma_{x}^{2}$ to the model of Eq. (6.20) for 10 turns injection.

parameters for all data sets. The parameters are plotted with respect to number of turns injected, conversion to total charge can be found in Fig. 6.1. The oscillation tune is 0.0065 at 10500 turn $(18.6 \mathrm{~ms})$ for 4-turn injection cycle, which is twice of the synchrotron tune $\nu_{s}=0.0034$ measured from turn-by-turn data at $18.4 \mathrm{~ms}$ with the same intensity.

The oscillatory part of $\sigma_{x}^{2}$ comes only from the longitudinal distribution. The 

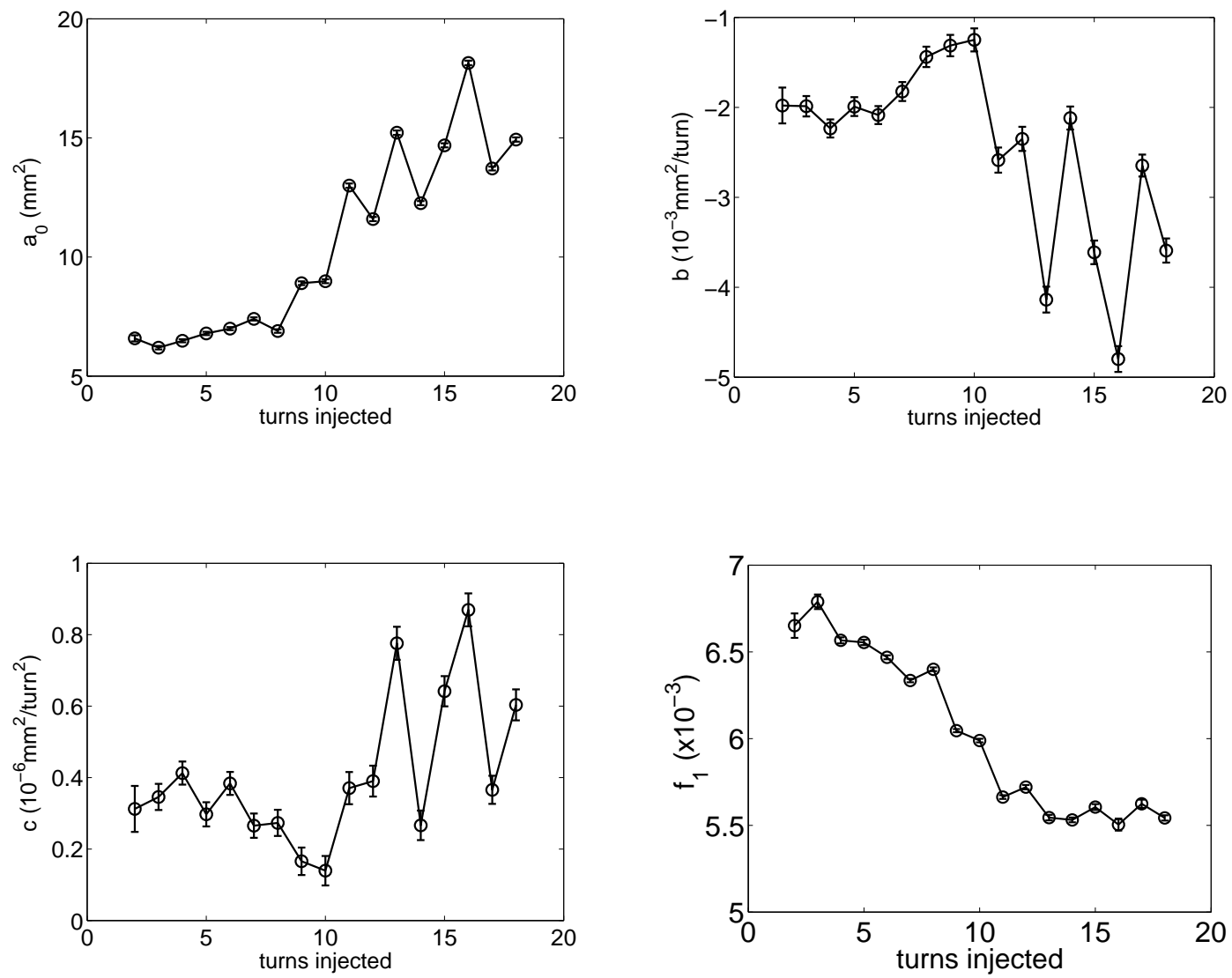

Figure 6.21: The fitting parameters of the post-transition horizontal beam size $\sigma_{x}^{2}$. Parameter $a\left(\mathrm{~mm}^{2}\right)$ at top left; parameter $b\left(\mathrm{~mm}^{2} /\right.$ turn $)$ at top right; parameter $c$ $\left(\mathrm{mm}^{2} / \mathrm{turn}^{2}\right)$ at bottom left; and the oscillation tune $f_{1}(1 /$ turn $)$ at bottom right. 

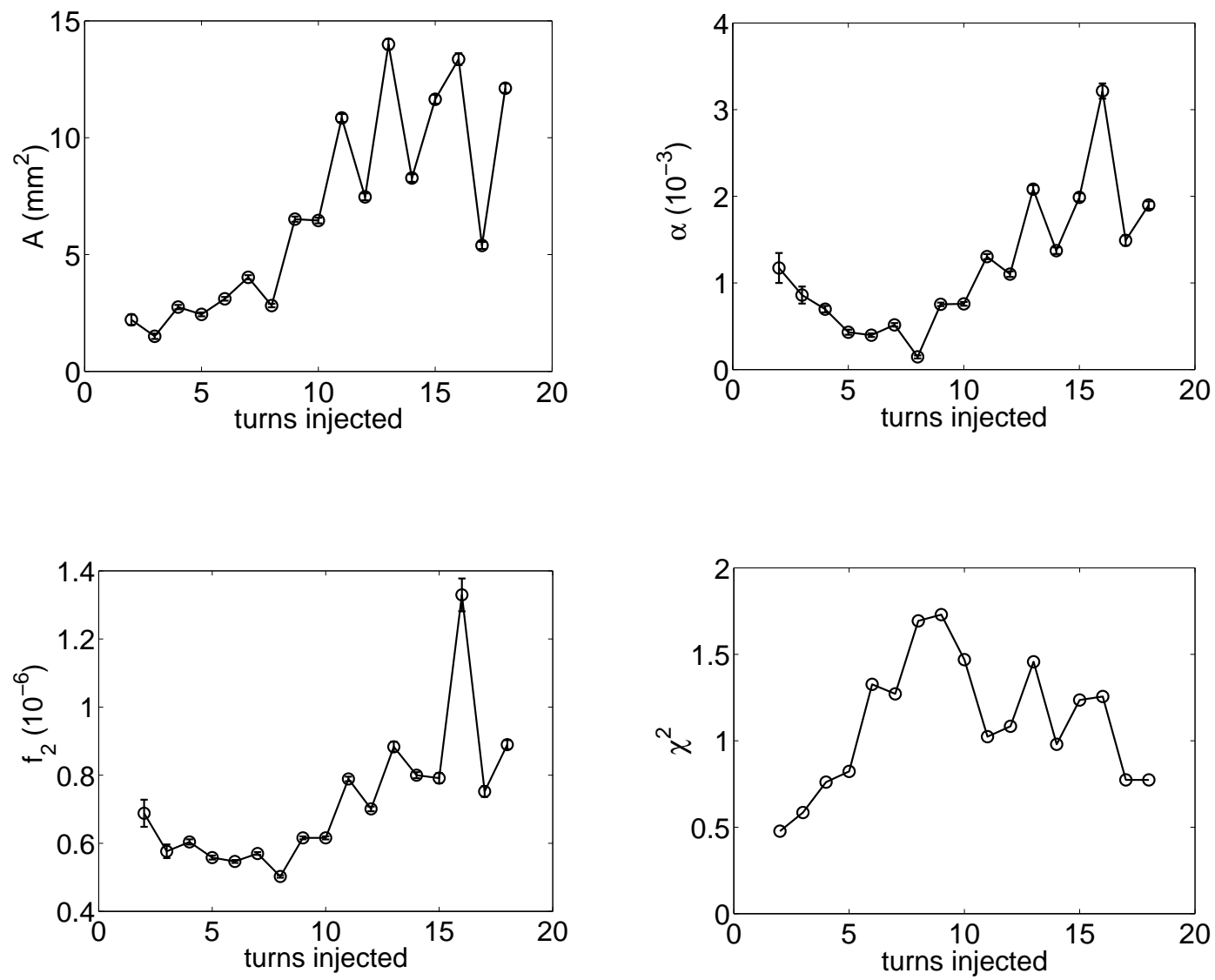

Figure 6.22: The fitting parameters of the post-transition oscillation of $\sigma_{x}^{2}$. Top left: the oscillation amplitude $A\left(\mathrm{~mm}^{2}\right)$; top right: the damping rate $\alpha(1 /$ turn $)$; bottom left: the drifting rate of oscillation tune $f_{2}$ $\left(1 /\right.$ turn $\left.^{2}\right)$; bottom right: the residual $\chi^{2}$ normalized by noise sigma and number of data points. 
resulting oscillation amplitude $A$ can be used to solve for phase space area by

$$
2 A=\sigma_{x, \max }^{2}-\sigma_{x, \min }^{2}
$$

Employing Eqs. (6.13), (6.15) and (6.16), we can self-consistently solve the mismatched motion. The resulting $\delta_{1}$ and $\delta_{2}$ are shown in Fig. 6.23.

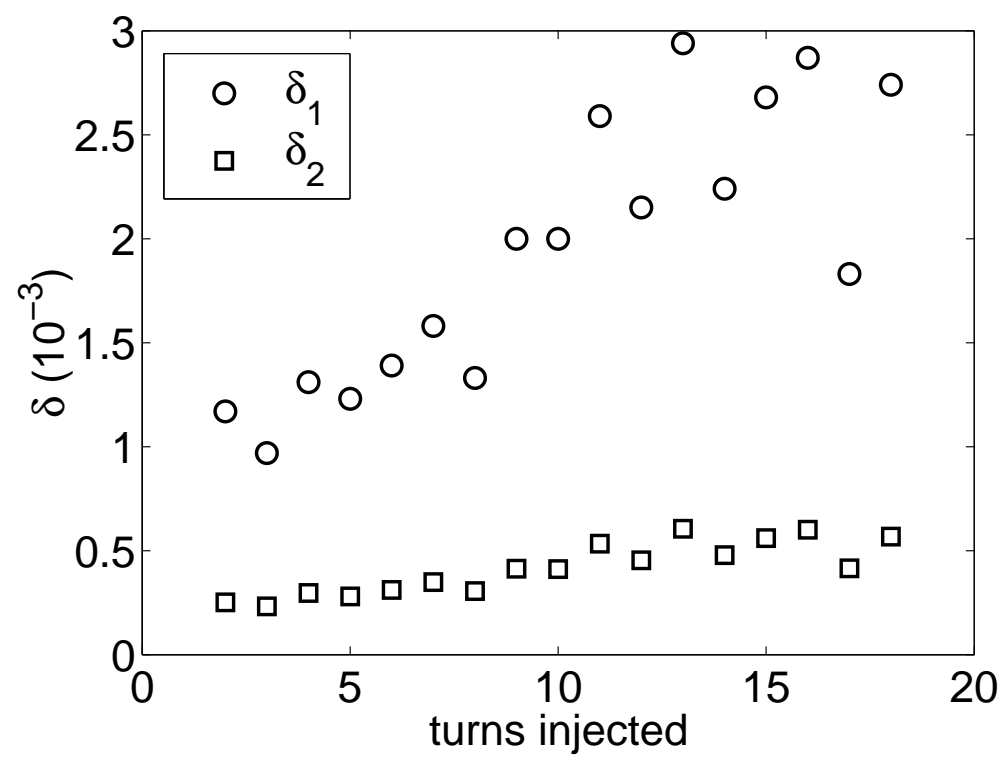

Figure 6.23: The maximum and minimum rms momentum width $\delta_{1}$ and $\delta_{2}$ calculated from the oscillation amplitude of $\sigma_{x}^{2}$

The non-oscillatory part

$$
\sigma_{x, \text { static }}^{2}=a+b t+c t^{2}
$$

is composed of transverse component and the static component of the momentum width. It can also be decomposed to the transverse and longitudinal components. Knowing the maximum $\left(\delta_{1}\right)$ and minimum $\left(\delta_{2}\right)$ of rms momentum width, the average 
value $\bar{\delta}$ can be calculated

$$
\bar{\delta}_{\mathrm{osci}}=\sqrt{\frac{\delta_{1}^{2}+\delta_{2}^{2}}{2}}
$$

where subscript "osci" indicates $\bar{\delta}$ is derived from the oscillation component of $\sigma_{x}^{2}$. Because the transition energy affects mainly the longitudinal motion, it is reasonable to assume that the transverse emittance will keep growing in the same manner as in the pre-transition region. The vertical emittance growth across transition (Fig. 6.6) suggests the same picture. Thus we have

$$
\begin{aligned}
\sigma_{x, \text { static }}^{2} & =\beta_{x} \epsilon_{x, \mathrm{rms}}+D^{2} \bar{\delta}^{2}=\frac{\beta_{x}}{\beta \gamma} \epsilon_{x, \mathrm{rms}}^{n}+D^{2} \bar{\delta}^{2} \\
& =\frac{\beta_{x}}{\beta \gamma} \epsilon_{x 0}^{n}\left(1+\alpha_{x} t\right)+D^{2} \bar{\delta}^{2}
\end{aligned}
$$

where $\epsilon_{x, \mathrm{rms}}^{n}$ is normalized rms emittance and $\alpha_{x}$ is horizontal emittance growth rate. The scaling rule is $\bar{\delta} \sim|\eta|^{-1 / 4} \gamma^{-3 / 4}$, neglecting the rf voltage $V$ factor which is constant in the concerned region. The scaling rule does not include the phase space dilution from the smearing of the mismatch bunch. By subtracting the predicted transverse component $\beta_{x} \epsilon_{x, \text { rms }}$ from $\sigma_{x \text {,static }}^{2}$, the rms momentum width can also be calculated by

$$
\bar{\delta}_{\text {static }}=\frac{\sqrt{\sigma_{x}^{2}-\beta_{x} \epsilon_{x, \mathrm{rms}}}}{D}
$$

where $D$ is the dispersion function at the IPM location. Using the growth rate obtained with the pre-transition fitting to predict $\epsilon_{x, \text { rms }}$ at turn 10501, we have calculated $\bar{\delta}_{\text {static }}$ for all data sets, which are compared to $\bar{\delta}_{\text {osci }}$ obtained with Eq. (6.22) in Fig. 6.24. It is seen that the two methods produce consistent results. 


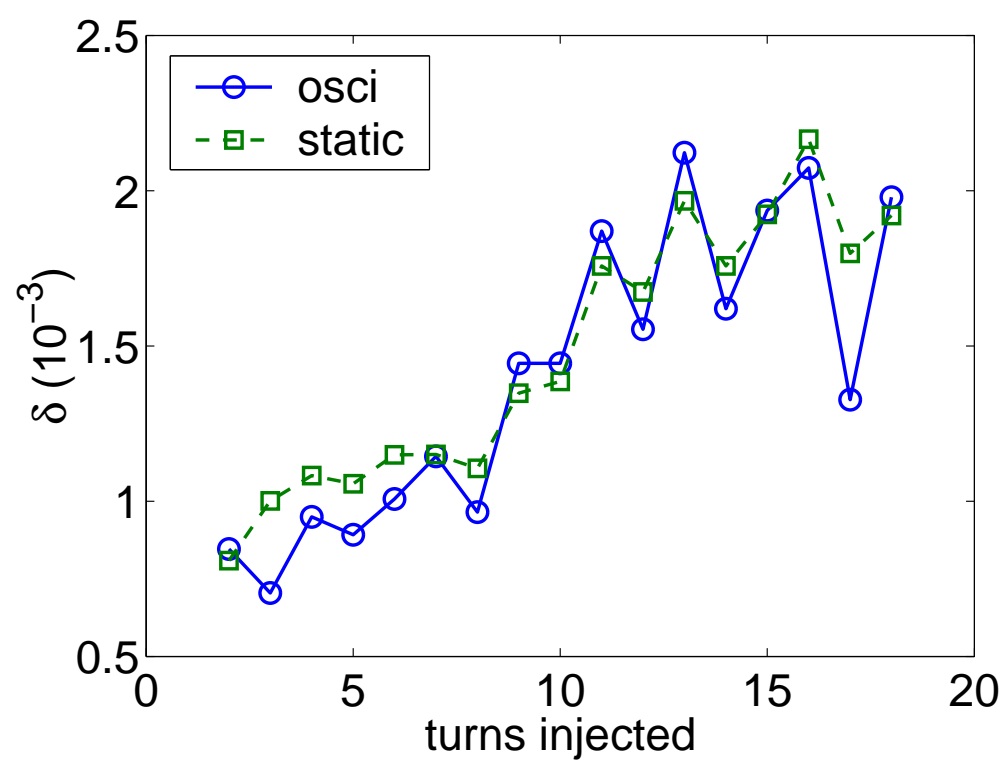

Figure 6.24: The average rms momentum width $\bar{\delta}$ obtained with two methods, Eq. (6.24) ("static") or Eq. (6.22) ("osci"). 


\subsection{Emittance Dilution and Other Observations}

\subsubsection{Effect of $\mathrm{rf}$ cavities at non-zero dispersive locations}

A mechanism that has not been studied carefully for emittance growth results from rapid energy gain in rf cavities that are located at non-zero dispersive region. The horizontal position of a particle is given by

$$
x=x_{\beta}+D \frac{\Delta E}{\beta^{2} E}, \quad x^{\prime}=x_{\beta}^{\prime}+D^{\prime} \frac{\Delta E}{\beta^{2} E},
$$

where $\left(x_{\beta}, x_{\beta}^{\prime}\right)$ are the betatron coordinates, and $\left(D, D^{\prime}\right)$ are the dispersion function. Particles gain energy in rf cavities. As the energy of a particle is gained in a cavity, the changes of the betatron coordinates are

$$
\Delta x_{\beta}=-D \frac{u}{\beta^{2} E}, \quad \Delta x_{\beta}^{\prime}=-D^{\prime} \frac{u}{\beta^{2} E},
$$

where $u$ is the energy gain at the cavity. The centroid of the beam is constantly and coherently excited by the betatron excitation. The bottom figure shows the betatron motion of the centroid of the beam for 400 revolutions with linear coupling.

We assume that the dipole magnets are ramped linearly, while the beam receives energy at cavities with energy $u$ in each cavity. The energy gain is about $400 \mathrm{keV}$ per revolution in the Booster. We define the fractional momentum deviation between the beam momentum $p_{\text {beam }}$ and the "magnet-momentum" $p_{\text {mag }}$ (the momentum associated with the magnetic field) as

$$
\frac{\Delta p}{p} \equiv \frac{p_{\text {beam }}-p_{\text {mag }}}{p_{\text {beam }}}
$$

The top plot of Fig. 6.25 shows that the beam momentum constantly oscillates against the momentum associated with the magnetic field. The effect of the coherent betatron oscillation on the beam emittance deserves a careful separate study. 

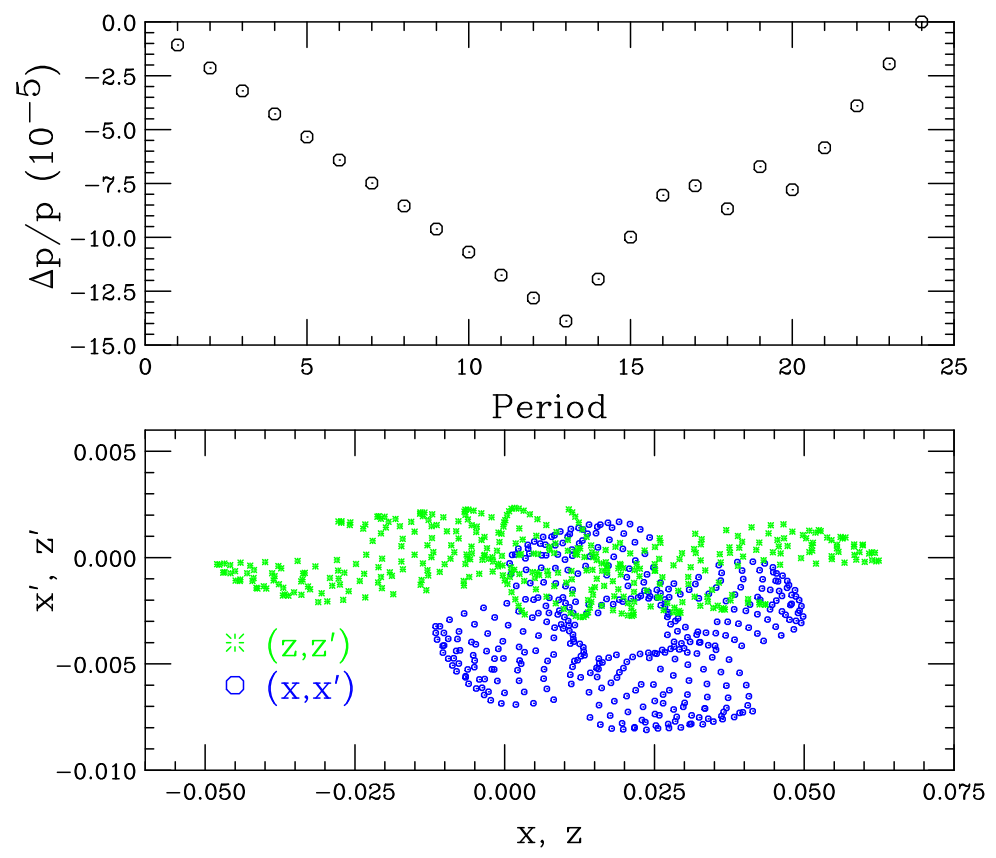

Figure 6.25: Bottom: The coherent betatron motion of the beam excited by the energy gain in each cavity. A linear coupling with a focal length of about $200 \mathrm{~m}$ is included in attaining the coherent betatron motion in the vertical plan. The units for $(x, z)$ and $\left(x^{\prime}, z^{\prime}\right)$ are $\mathrm{mm}$ and $\mathrm{m}$-rad respectively. Top: The fractional momentum deviation of the beam with respect to the momentum associated with the dipole magnetic field. 


\subsubsection{Other observations}

Other interesting observations in our measurements include the horizontal beam width oscillation before transition as can be seen in the bottom right subplot (14-turn injection) of Fig. 6.16 and the post-transition vertical beam width oscillations. The vertical emittance post-transition oscillations are found to be out of phase with the horizontal emittance by $\pi$, which is not understood. These observations are left for future exploration.

\subsection{Space Charge Half-integer Stopband Correc- tion}

It is well known that the space charge effect causes emittance growth in low energy synchrotrons. This is observed in our measurements with the Booster as shown in Fig. 6.9. The emittance grows fast for low-energy and high-intensity beams, which is a clear sign of space charge effect. This process can now be simulated with several tracking codes which use the particle-in-cell (PIC) model. However it is still not understood theoretically. Ref [1] suggests a possible mechanism of the space charge induced emittance growth. It relates emittance growth to resonant excitations of the envelope oscillation of mismatched beam. Based on this model, we speculate that emittance growth would be reduced if we adjust the lattice model to cancel the resonant envelope stopband integral. This approach is called the space charge stopband correction.

In this section we describe a method to correct the resonant stopband integrals and demonstrates it with the Fermilab Booster model. We first briefly summarize the theory of resonant envelope oscillation and stopband integrals as in Ref [1]. 


\subsubsection{Envelope oscillation and half-integer stopband}

We use a KV beam to illustrate the envelope oscillation theory. The envelope equation of a $\mathrm{KV}$ beam is given by

$$
R_{\mathrm{b}}^{\prime \prime}+k(s) R_{\mathrm{b}}-\frac{\epsilon^{2}}{R_{\mathrm{b}}^{3}}-\frac{K_{\mathrm{sc}}}{R_{\mathrm{b}}}=0,
$$

where $k(s)$ is the focusing function, $\epsilon$ is beam emittance, $R_{\mathrm{b}}$ is the beam envelope radius $\left(R_{\mathrm{b}}=\sqrt{\beta(s) \epsilon}\right.$ for matched beam), $K_{\mathrm{sc}}$ is the space charge perveance parameter and $\beta(s)$ is the betatron amplitude function. Making Floquet transformation with

$$
R=\frac{R_{\mathrm{b}}}{\sqrt{\beta(s) \epsilon}}, \quad \phi=\frac{1}{\nu} \int_{0}^{s} \frac{d s}{\beta(s)},
$$

where $\nu$ is the betatron tune, we transform the envelope equation to

$$
\ddot{R}+\nu^{2} R-\frac{\nu^{2}}{R^{3}}-\frac{\nu^{2} \beta(s) K_{\mathrm{sc}}}{\epsilon R}=0
$$

with new time-coordinate $\phi$.

Now we consider the space charge term as a small perturbation. We can expand the actual envelope radius around its unperturbed counterpart to $R=1+r+\Delta$ with a static ( $\phi$-independent) term $\Delta$ and an oscillatory ( $\phi$-dependent) term $r$. Expanding the space-charge factor

$$
\frac{\nu \beta(s) K_{\mathrm{sc}}}{2 \epsilon}=\xi_{\mathrm{sc}}\left(1+\sum_{n=1}^{\infty} q_{n} \cos \left(n \phi+\chi_{n}\right)\right)
$$

into Fourier series, with

$$
\begin{aligned}
\xi_{\mathrm{sc}} & =\frac{1}{2 \pi} \oint \frac{\nu \beta K_{\mathrm{sc}}}{2 \epsilon} d \phi=\frac{K_{\mathrm{sc}} C}{4 \pi \epsilon}, \\
\xi_{\mathrm{sc}} q_{n} & =\frac{1}{\pi} \oint \frac{\nu \beta K_{\mathrm{sc}}}{2 \epsilon} \cos \left(n \phi+\chi_{n}\right) d \phi,
\end{aligned}
$$

where $C$ is the circumference of the ring. Inserting the Fourier expanded factor into the envelope equation, we get $\Delta=\xi_{\mathrm{sc}} / 2 \nu$ and

$$
\ddot{r}+\left(4 \nu^{2}-4 \nu \xi_{\mathrm{sc}}\right) r \approx 2 \nu \xi_{\mathrm{sc}} \sum_{n=1}^{\infty} q_{n} \cos \left(n \phi+\chi_{n}\right) .
$$


Eq. (6.33) shows that the envelope radius can be resonantly excited by the space charge perturbation if the envelope tune $\nu_{\mathrm{env}}=2 \nu-\xi_{\mathrm{sc}}$ is close to an integer. In that case we get

$$
r \approx \frac{2 \nu \xi_{\mathrm{sc}} q_{n}}{-n^{2}+\left(4 \nu^{2}-4 \nu \xi_{\mathrm{sc}}\right)} \cos \left(n \phi+\chi_{n}\right)
$$

with integer $n \approx \nu_{\text {env }}$.

When the envelope is resonantly excited, the betatron phase space mismatch becomes severe. Some particles can be left out of the beam core which then lead to emittance dilution. An efficient way to reduce the emittance growth is thus to reduce the resonant stopband integral $\xi_{\mathrm{sc}} q_{n}$.

\subsubsection{Stopband correction}

Rewriting Eq. (6.32) into the form

$$
\begin{aligned}
q_{n} & =\frac{1}{\pi} \frac{K_{\mathrm{sc}}}{2 \epsilon \xi_{\mathrm{sc}}} \oint \cos \left(n \phi+\chi_{n}\right) d s \\
& =\frac{2}{C} \oint \cos \left(n \phi+\chi_{n}\right) d s,
\end{aligned}
$$

we see that the stopband arises only from the non-uniform spatial distribution of betatron phase advance. Since the trim quadrupoles perturb the betatron amplitude function as well as the betatron phase advance around the ring, the stopband integrals can be corrected by properly adjusting the trim quadrupoles.

The perturbation to beta function due to trim quadrupoles is

$$
\frac{\Delta \beta}{\beta}=-\frac{\nu_{0}}{2} \sum_{p} \frac{j_{p}}{\nu_{0}^{2}-(p / 2)^{2}} e^{j p \phi}
$$

where $j_{p}$ is the $p$ 'th half-integer stopband integrals for quadrupoles defined as

$$
j_{p}=\frac{1}{2 \pi} \oint k(s) \beta(s) e^{-j p \phi} d s=\frac{1}{2 \pi} \sum_{i}[k \Delta l]_{i} \beta_{i} e^{-j p \phi_{i}}
$$




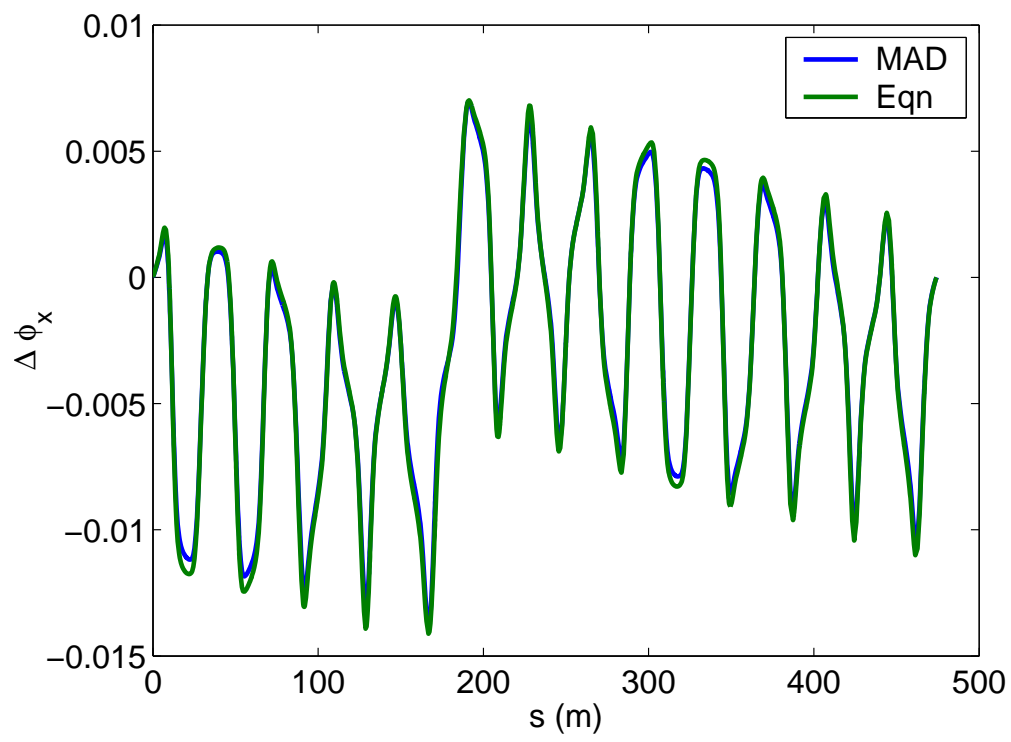

Figure 6.26: (Color) Comparison of $\Delta \phi_{x}$ due to an increase of QS-9 by $0.5 \mathrm{~A}$ with MAD calculation and Eq. (6.38) (using 20 harmonics). Eq. (6.38) is verified.

and the perturbation to phase advance is

$$
\begin{aligned}
\Delta \phi & =\frac{1}{\nu_{0}} \Delta\left(\int_{0}^{L} \frac{d s}{\beta}\right)=-\frac{1}{\nu_{0}} \int_{0}^{L} \frac{\Delta \beta}{\beta} \frac{d s}{\beta} \\
& =-\int_{0}^{\phi} \frac{\Delta \beta}{\beta} d \phi^{\prime}=\frac{\nu_{0}}{2} \sum_{p} \frac{j_{p}}{\nu_{0}^{2}-(p / 2)^{2}} \int_{0}^{\phi} e^{j p \phi^{\prime}} d \phi^{\prime} \\
& =\frac{\nu_{0}}{2} \sum_{p} \frac{j_{p}}{\nu_{0}^{2}-(p / 2)^{2}} \frac{1}{j p}\left(e^{j p \phi}-1\right) .
\end{aligned}
$$

Eq. (6.38) is verified by comparing $\Delta \phi$ due to one trim quad with MAD calculation and the equation. The comparison is shown in Fig 6.26. 
Thus the perturbation to the stopband integral $q_{n}$ is

$$
\begin{aligned}
\Delta q_{n} & =-\frac{2 n}{C} \oint \sin \left(n \phi+\chi_{n}\right) \Delta \phi d s \\
& =\frac{n \nu}{2 C} \sum_{p} \frac{j_{p}}{\nu^{2}-(p / 2)^{2}} \frac{1}{p} \oint\left(e^{j\left(n \phi+\chi_{n}\right)}-e^{-j\left(n \phi+\chi_{n}\right)}\right)\left(e^{j p \phi}-1\right) d s \\
& =-\frac{\nu}{2\left(\nu^{2}-(n / 2)^{2}\right)}\left(j_{n} e^{-j \chi_{n}}+j_{-n} e^{j \chi_{n}}\right),
\end{aligned}
$$

where in the last step we keep only terms with $p= \pm n$. For each trim quadrupole, $j_{ \pm n}=\frac{1}{2 \pi}[k \Delta l] \beta e^{\mp j n \phi}$, hence the correction of the resonant stopband integral due to the $i$ 'th trim quadrupole is

$$
\Delta q_{n}^{i}=-\frac{\nu}{\nu^{2}-(n / 2)^{2}} \frac{[k \Delta l]_{i} \beta_{i}}{2 \pi} \cos \left(n \phi_{i}+\chi_{n}\right) .
$$

Eq. (6.40) is also verified with the Booster lattice. For example, with the operation setting when data in Fig. 6.9 were taken the model gives $q_{x, 13}=0.1494$ and $\Delta q_{x, 13}^{S 1}=$ 0.0866 for a change of $1.0 \mathrm{~A}$ of trim quadrupole S1. Reducing the current of trim quadrupole S1 by $0.2 \mathrm{~A}$ and re-evaluating the stopband integral we get $q_{x, 13}=0.1338$. The change of $q_{x, 13}$ is -0.0156 , compared to $0.0866 \times(-0.2)=-0.0173$. For the same setting, $q_{z, 14}=0.1208$ and $\Delta q_{z, 14}^{L 2}=0.0619$. Reducing trim quadrupole L2 by $0.2 \mathrm{~A}$ brings $q_{z, 14}$ down to 0.1079 , which also verifies the equation.

In practice, we want to correct stopband integrals for both the horizontal and vertical planes. And it is desirable to compensate the two harmonics nearest to $\nu_{\mathrm{env}}$. For example, for the Booster, we want to compensate $q_{x, 13}, q_{x, 14}, q_{z, 13}$ and $q_{z, 14}$. A working correction scheme needs to consider all these harmonics. It can be done by defining a merit function which include all 4 harmonics

$$
f=q_{x, 13}^{2}+q_{x, 14}^{2}+q_{z, 13}^{2}+q_{z, 14}^{2}
$$

with

$$
q_{y, n}=q_{y, n 0}+\sum_{i} \Delta q_{n}^{i} \Delta I_{i},
$$


where $y$ stands for $x$ or $z$ and $\Delta I_{i}$ is the change of the $i$ 'th trim quadrupole. Using the gradient of function $f$, it is very easy to reduce it, along with all related harmonics. The stopband correction for the Booster is described in the next sub-section.

\subsubsection{Stopband correction for the Booster}

For the Booster, the nominal betatron tunes are $\nu_{x}=6.7$ and $\nu_{z}=6.8$, which make the 13rd and 14th harmonics most dangerous. Unfortunately, these harmonics are unusually large, as shown in Fig. 6.27 (left), which shows the stopband integrals with the recorded setting for data in Fig. 6.9. It is seen that $q_{x, 13}$ and $q_{z, 14}$ are much higher than other harmonics.

Using the stopband correction method described in the last sub-section, we have successfully reduced these stopband integrals to a very low level. The corrected result is shown in Fig. 6.27 (right) which shows that $q_{x, 13}$ and $q_{z, 14}$ are down to 0.01. The harmonics before and after correction are also shown in Table 6.1. If the envelope oscillation is indeed the cause of emittance growth, such correction of envelope stopband would dramatically improve the beam performance. The correction setting only changes half of the trim quadrupoles by up to $0.4 \mathrm{~A}$.

Table 6.1: The concerned harmonics before and after correction

\begin{tabular}{|l|l|l|l|l|}
\hline & $q_{x, 13}$ & $q_{x, 14}$ & $q_{z, 13}$ & $q_{z, 14}$ \\
\hline initial & 0.1494 & 0.0954 & 0.0207 & 0.1208 \\
corrected & 0.0095 & 0.0316 & 0.0058 & 0.0129 \\
\hline
\end{tabular}

A big concern in practice is of course the sensitivity of the calculated correction 

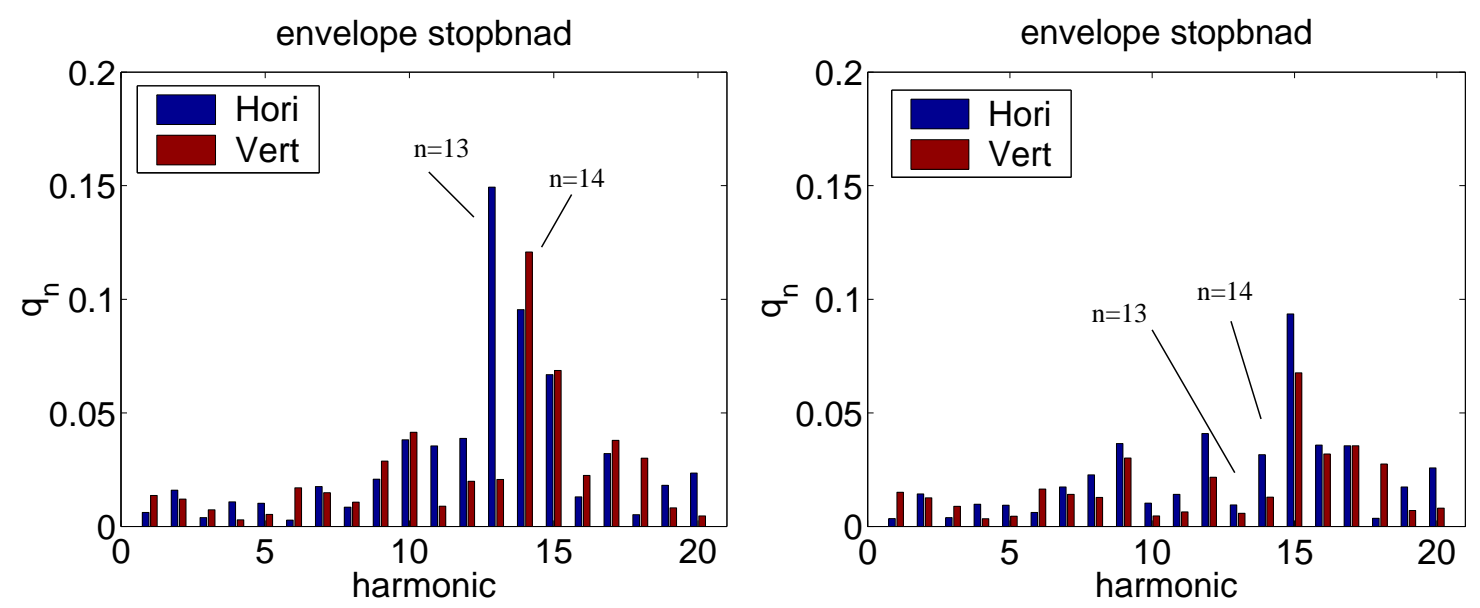

Figure 6.27: Left: the envelope stopband integrals for the Booster with recorded operation settings. Right: the corrected envelope stopband integrals.

setting. The maximum sensitivity is estimated by

$$
\left|\frac{\Delta q_{n}^{i}}{\Delta I_{i}}\right| \leq\left|\frac{\nu}{\nu^{2}-(n / 2)^{2}} \frac{[k \Delta l]_{i} \beta_{i}}{2 \pi}\right|
$$

according to Eq. (6.40). Using $\nu_{x}=6.7, k \Delta l=0.0068 \mathrm{~m}^{-1}$ for $1 \mathrm{~A}$ at $400 \mathrm{MeV}$, $\beta_{x}=34.0 \mathrm{~m}$ in average at short sections, we get a maximum change of $q_{x, 13}$ of 0.094 by 1 A change of current of a short section trim quadrupole. Using $\nu_{z}=6.8, \beta_{z}=21.0 \mathrm{~m}$ at long sections in average, the maximum sensitivity is 0.056 by $1 \mathrm{~A}$ change of a long section trim quadrupole. Such sensitivity level are within the controllable range in experiments. However, one has to have an accurate lattice model to find a meaningful correction setting. We expect that the model should be accurate enough to assure the error of the calculated lattice functions (beta function and phase advance) are within the error level due to $1 \mathrm{~A}$ change of one trim quadrupole. This is beyond the model accuracy we currently have. 


\subsection{Summary}

We have measured the Booster beam profile under various beam intensity levels. The normalized vertical emittance has different growth behavior in the first several milliseconds and later in the cycle. Our findings can be summarized as follows.

1. The normalized vertical emittance starts at about $2 \pi$-mm-mrad for all intensity levels. The emittance grows rapidly in the first 3000 turns where the space charge effect is important. The first-stage emittance growth is particularly important when the beam intensity is larger than 12-turn injection. For 13-turn injection or higher intensity, emittance grows rapidly in the first few hundreds of turns in a manner that is far different from the lower intensity data sets. Such observation implies a intensity threshold beyond which the emittance growth mechanism changes suddenly. A possible half integer stopband compensation for the space charge effects is contemplated. It is based on the theory that the emittance growth of space-charge dominated beams occurs through resonant envelope oscillations due to mismatch. We propose a stopband correction scheme to compensate the space charge half-integer stopband width as a measure to alleviate emittance growth and beam loss. Although high accuracy of the lattice model is required to calculate the working point of correction, the successful application of the approach is foreseeable.

2. Later in the cycle, the emittance grows linearly with a growth rate of about $1 \pi$-mm-mrad in $10^{4}$ turns. Both the intra-beam scattering and the beam-gas scattering growth rates are too small to explain this linear emittance growth rate. We note that the beam is constantly and coherently excited by the energy gain in passing through the cavity. Can this excitation combined with beam decoherence produce the linear emittance growth? It deserves a careful study. 
3. The horizontal beam width is composed of both the transverse and the longitudinal phase space distribution of the beam. Making use of the fact that the two components possess different time dependence, we have separated them by fitting $\sigma_{x}^{2}$ to three terms with different scaling rules. Such fitting works well for data sets with less than 10-turn injection when the calibration error is small. The fitting results yield horizontal normalized emittance and the rms momentum width. The growth rate of the normalized emittance is found to be about $0.8 \pi$-mm-mrad in $10^{4}$ turns.

4. The horizontal beam width starts to oscillate with frequency twice the synchrotron tune (quadrupole mode) after transition. This oscillation comes from the mismatch between beam bunch and longitudinal phase space ellipse. The mismatch was commonly believed to be due to the longitudinal space charge effect which is de-focusing before transition and focusing after transition. However, our estimation of space charge effect indicates it is relatively small (below $2 \%$ of the rf voltage). This is consistent with our modeling result which assumes the mismatch comes only from non-adiabatic motion near transition. We can fit the bunch shape mismatch with the phase shape distortion due to non-adiabatic motion near transition. We extracted the oscillatory and non-oscillatory parts of $\sigma_{x}^{2}$ by fitting it to a model with second-order polynomial plus damped oscillation with linear phase shift rate. The maximum and minimum rms momentum deviation are estimated using the oscillation amplitude of $\sigma_{x}^{2}$. The non-oscillatory part is separated to horizontal emittance and static (average) rms momentum deviation by assuming the horizontal normalized emittance grows linearly as in the region before transition. The estimated average rms momentum widths with both the oscillatory or non-oscillatory parts are consistent. The post-transition bunch length (and beam size) oscillation is a possible cause of beam loss at 
transition. We would like to point out that the bunch shape mis-match can be compensated by rf voltage modulation at a frequency slightly larger than twice the synchrotron frequency [51]. It is estimated that a voltage modulation depth of about $3 \%$ of the total rf cavity voltage, i.e. about $24 \mathrm{kV}$, is sufficient. 


\section{Chapter 7}

\section{Conclusions and Discussions}

In this thesis we studied the machine lattice of the Fermilab Booster with beambased measurements in order to understand the beam loss. The orbit response matrix (ORM) was used to calibrate the existing lattice model. In spite of the difficulties due to the nature of the machine, we have managed to extract an equivalent lattice model and obtained consistent results on the gradient errors of the Booster. The independent component analysis is introduced as a beam diagnostics method for synchrotron BPM turn-by-turn data. It proved to be a powerful tool through simulation studies and the application to data taken at the Booster. Emittance growth in the Booster is also studied and an approach to cure the early emittance growth is proposed.

We have applied the standard ORM approach to calibrate the lattice model of the Booster. However it did not yield sensible results. The fitting solution was not unique. The gradient errors were found to be larger than $10 \%$ of nominal gradients with spurious symmetric pattern between the fitted model parameters of adjacent magnets. This was because these adjacent magnets perturb the lattice in very similar patterns so that the ORM does not have the necessary information to distinguish them. Suitable constraints were introduced to remove the ambiguity of the model parameters which 
then led to a robust, unique, equivalent lattice model as represented by the ORM data. Even though the individual gradient parameters cannot be determined, simple combinations (sums) of them are preserved by the constrained fitting scheme, i.e., the combinations of the fitted parameters reflect that of the real machine. It is found the gradient errors are generally within $1 \%$ of the nominal gradient values for the focusing magnets, which is the design tolerance. There is a clear linear correlation between the gradient errors and the horizontal beam orbit offsets. This is strong evidence that the gradient errors we found are due to sextupole focusing of the body sextupole components of the main magnets.

Multiple BPM turn-by-turn data taken while coherent motion of the beam is excited contains a vast amount of information on the machine. Data mining plays an important role in uncovering the useful information. In this study, the independent component analysis (ICA) is first introduced to analyze synchrotron BPM turn-byturn data. The readings of the BPMs are considered as linear mixtures of several independent source signals plus random noise. The source signals are assumed to be narrow-band signals with non-overlapping spectra. Consequently the time-lagged covariance matrices of the signals are diagonal. The ICA method isolates the independent sources by jointly diagonalizing the time-lagged covariance matrices. The betatron motion, synchrotron motion and other potential perturbation sources can then be identified and studied separately. From the spatial patterns of the extracted betatron motion, beta functions and betatron phase advances can be measured. The temporal patten is a clean signal which can be used to measure the betatron tunes to high accuracy with advanced tune evaluation method such as the interpolated FFT. The separated synchrotron motion can be used for dispersion function measurement and also for the study of machine properties that are related with the longitudinal motion. Compared to the earlier method, the model independent analysis (MIA) $[16,17,18]$, the ICA method has the same effect of noise reduction because both 
methods use principal component analysis (PCA) to identify and attain the nontrivial components. However, the ICA method gains better ability in mode separation by making use of additional information, i.e., the statistical independence of the source signals. The ICA method has the capability to separate weak perturbation signals that are present in BPM data. It is robust over the contamination of bad BPMs, which makes it suitable for online application. Another advantage of the ICA method is that it deals with linear coupling properly while MIA does not [17]. The ICA method isolates the coupled betatron motion into normal modes as stated in Edwards-Teng's parameterization theory [35]. This improves the accuracy of beta function and phase advance measurements. Simulation studies have been carried out to verify and explore the capability of the ICA method.

We have applied the ICA method to data taken at the Booster for various studies. The linear lattice functions were measured and the results were used to verify the existing lattice model. It is found that the discrepancy of horizontal and vertical beta function, measured with average $\Delta \beta / \beta$, is about $12 \%$. The average difference of phase advance from BPM to BPM between the model and the measurement is about $0.10 \mathrm{rad}$. The imaginary part of transverse impedance $\operatorname{Im} Z_{\perp}$ of the Booster is measured through the tune shifts due to increased beam intensity. The result of $\operatorname{Im} Z_{\perp}$ is nearly $50 \mathrm{M} \Omega / \mathrm{m}$ at injection and falls approximately with the $1 / B_{f}$ curve. The measured transverse impedance is partially explained by the image current in magnet poles and the image charge in vacuum pipes. The chromaticity throughout the Booster cycle is also measured through the tune shifts due to changes of the radial position of the beam. Synchrotron motion is observed in the BPM data. The synchrotron oscillation is found to be very weak. However, the synchrotron tune signal is obtained in the entire cycle.

The emittance growth provides insights into the beam loss mechanism. The transverse emittance is studied via data taken with the ion profile monitor (IPM) for the 
entire Booster cycle, especially in the region after injection and across transition. A linear emittance growth rate as large as $1 \pi$-mm-mrad per $10^{4}$ turns for both transverse planes is found for a Booster cycle with normal operation intensity. It is far larger than the estimated growth rates due to either intrabeam scattering or background gas scattering. Coherent horizontal kicks the beam receives from discrete energy gain at the rf cavities and subsequent decoherence could provide an explanation. The emittance growth in space-charge-dominated region is also studied. It is observed that the emittance grows violently above a certain intensity threshold in the first few hundreds turns. Below the threshold the growth rate is mild and can be described by a growth model that is linear with respect to the space charge perveance parameter. The transverse emittance and longitudinal momentum spread contributions to the horizontal beam sizes are separated with a fitting model that exploits the different scaling rules of the two components. The results are consistent with other observations, including a separate longitudinal momentum spread measurements. The longitudinal phase space dilution due to non-linear longitudinal motion at transition and the microwave instability are estimated. The post-transition quadrupole mode oscillations of horizontal beam size due to longitudinal phase space mismatch are studied. It is found that the longitudinal space charge effect is not as important as commonly believed. The phase space mismatch is explained by considering only the non-adiabatic longitudinal motion near transition. The space charge half-integer stopband correction, an approach to cure the space-charge-induced emittance growth of low-energy synchrotron beams is proposed.

This study can be continued in several directions to expand the application of the data analysis method and to gain further insights of the Booster to benefit its operation and reduce beam losses.

In the current ORM study, the linear correlation between the fitted gradient errors and the natural horizontal orbit shifts due to cycling is examined. It is possible to 
study the sextupole components deliberately by creating local orbit bumps between different ORM data sets.

The ICA method in this study is suitable only for synchrotron turn-by-turn data because time correlation of the source signals is assumed. It cannot be applied to linac pulse-by-pulse data as the PCA-based MIA. However, the ICA method is a broad category of data analysis techniques. The ICA methods that are based on the non-gaussianity of the latent variables could be used for linac pulse-by-pulse data because the underlying physical variables are statistically independent and very likely obey non-gaussian distributions.

In the application of the ICA method to the Booster we have measured the imaginary part of the transverse impedance. Its source is not fully understood yet. We need to carefully consider the contribution of the resistive wall impedance of the laminated magnets, which have currently been neglected. The image charge effect in magnets poles also needs to be considered. It is desirable to study the real part of the transverse impedance using transverse instability which can be observed in DC mode operation.

We have tried the approach of reducing emittance growth with space charge resonance stopband correction in the Booster. There was no clear effect when the calculated correction setting is dialed in on the console. However, this does not mean this approach would not work. We believe the negative result is due to the accuracy of the model we used for correction setting. The calculation is not good enough because the sextupole focusing effect is very difficult to predict or control. In future experiments, two measures could be taken to obtain better results. One may take a complete ORM data set and use it to calibrate the lattice model and use the calibrated model for correction setting calculation. A better approach is to combine the trim quadrupoles into a few groups and fine-tune the settings of these groups with knobs to scan over a large area of the parameter space. It seems possible to devise a scheme to organize 
the knobs and use a tuning strategy to find the working point corresponding to the best correction setting.

The post-transition bunch length oscillation can be reduced by rf voltage modulation. Simulation studies are needed to learn the modulation parameters which can effectively damp out the oscillation. 


\section{Appendix A}

\section{Booster kinematic parameters}

The ramping parameters such as beam energy, beam velocity, rf frequency, etc. can be derived from Eq. (1.1). Knowing that the kinetic energy is $400 \mathrm{MeV}$ at injection and $8 \mathrm{GeV}$ at extraction, we have

$$
\frac{P}{m_{0} c}=5.24537-4.22833 \cos (30 \pi t)
$$

which leads to other ramping parameters. The phase slip factor $\eta$ is obtained using nominal transition gamma $\gamma_{T}=5.446$. Some parameters are shown in Fig. A.1, A.2 and are listed in Table A.1. Typical rf operation setting and relevant parameters (e.g., bucket area) can be found in chapter 6 . 

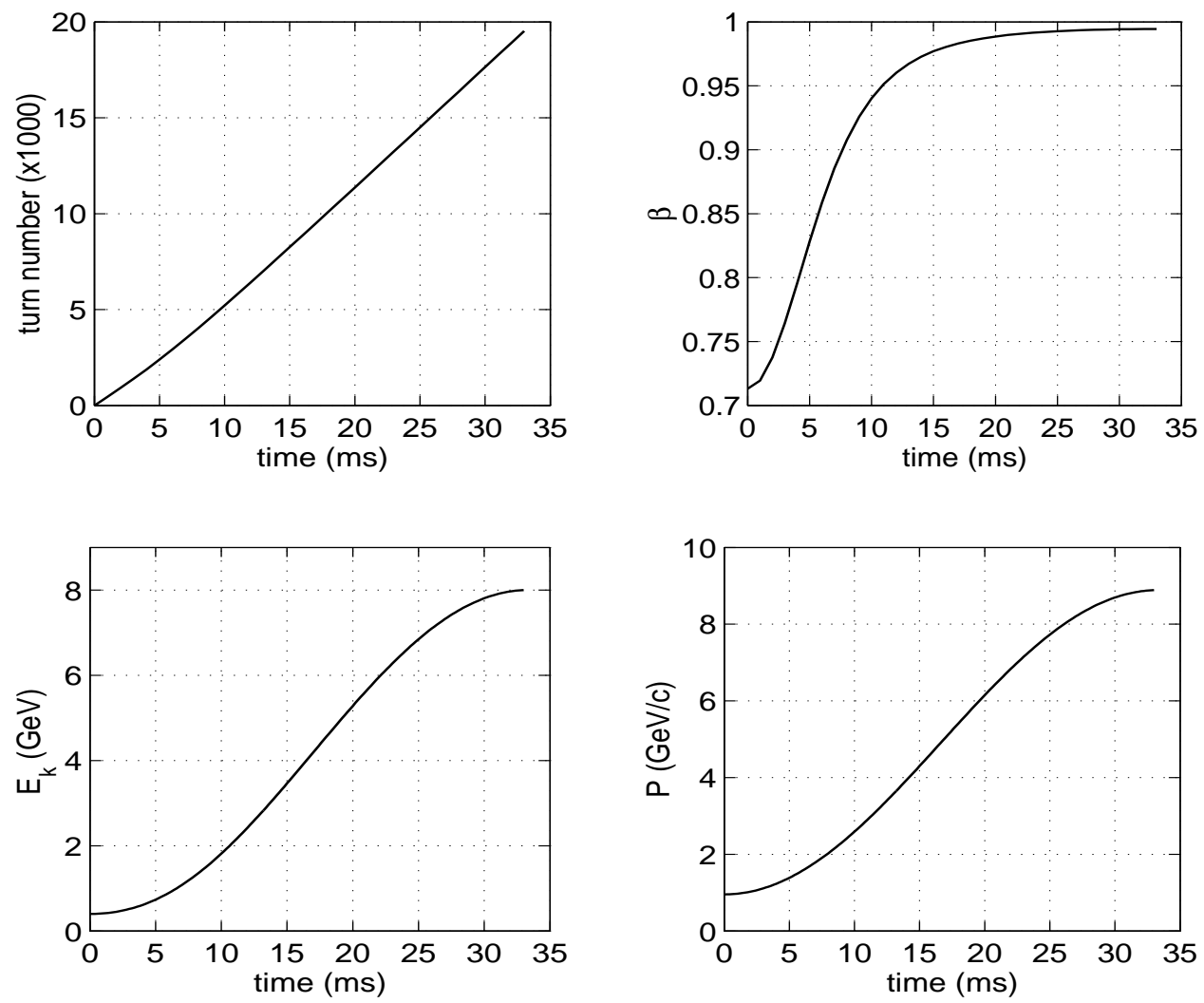

Figure A.1: The ramping kinematic parameters of the Booster.

Top left: turn number vs. cycling time. Top right: relativistic $\beta$ of the beam. Bottom left: the kinetic energy $E_{k}(\mathrm{GeV})$. Bottom right: the beam momentum $P(\mathrm{GeV} / \mathrm{c})$. 

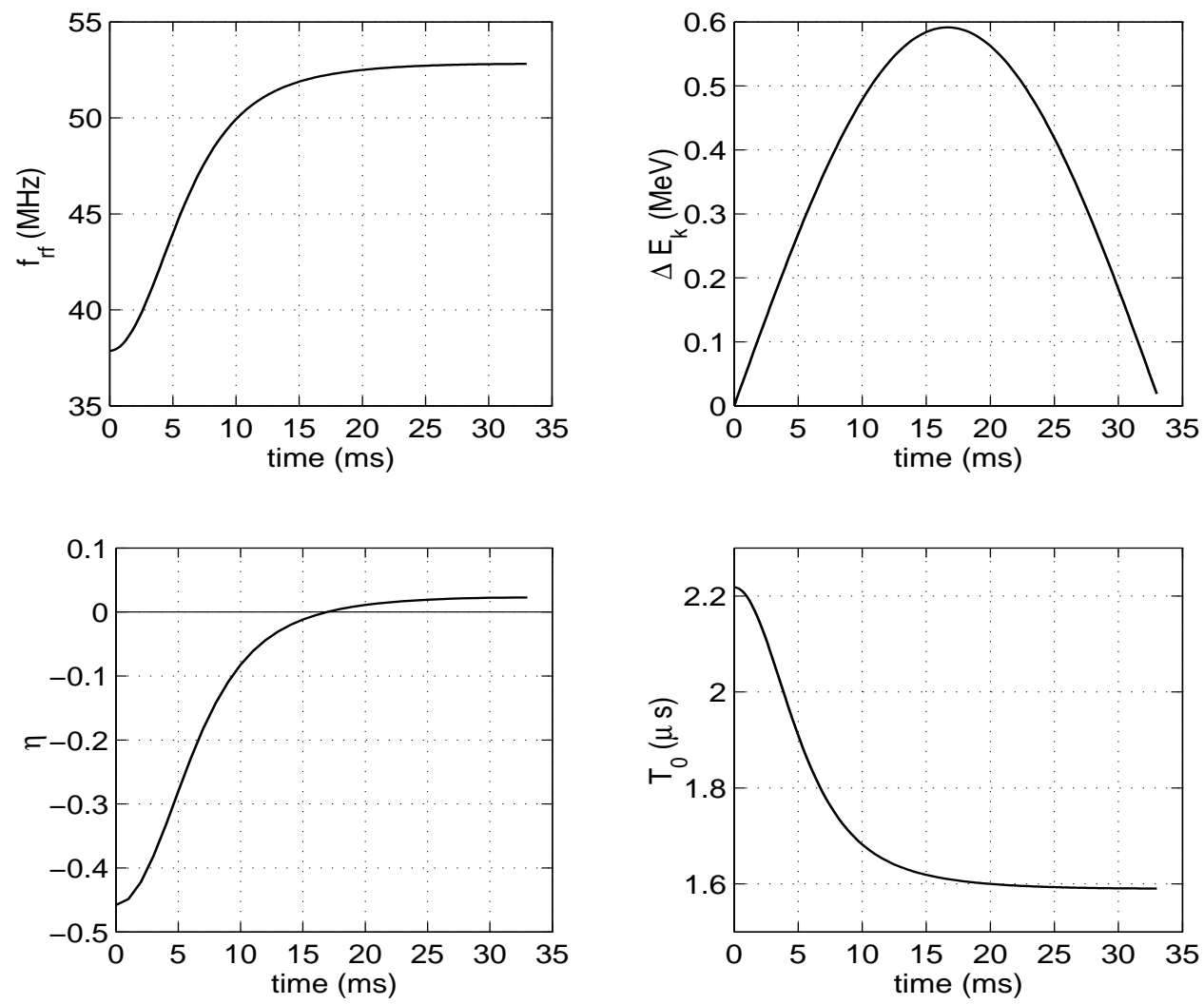

Figure A.2: The ramping kinematic parameters of the Booster continued. Top left: rf frequency $(\mathrm{MHz})$. Top right: energy gain per revolution $\Delta E_{k}$. Bottom left: the phase slip factor $\eta$. Bottom right: the revolution time $T_{0}(\mu \mathrm{s})$. 


\begin{tabular}{|c|c|c|c|c|c|c|c|c|c|}
\hline time $(\mathrm{ms})$ & turn & $\beta$ & $\gamma$ & $E_{k}(\mathrm{GeV})$ & $P(\mathrm{GeV} / \mathrm{c})$ & $\eta$ & $f_{\mathrm{rf}}(\mathrm{MHz})$ & $T_{0}(\mu \mathrm{s})$ & $\Delta E_{k}(\mathrm{MeV})$ \\
\hline 0 & 0 & 0.7131 & 1.4263 & 0.4000 & 0.9543 & -0.4578 & 37.8677 & 2.2183 & 0.0000 \\
\hline 1 & 452 & 0.7194 & 1.4398 & 0.4126 & 0.9719 & -0.4487 & 38.2063 & 2.1986 & 0.0557 \\
\hline 2 & 912 & 0.7375 & 1.4806 & 0.4510 & 1.0245 & -0.4224 & 39.1643 & 2.1448 & 0.1108 \\
\hline 3 & 1386 & 0.7642 & 1.5505 & 0.5165 & 1.1118 & -0.3822 & 40.5851 & 2.0697 & 0.1650 \\
\hline 4 & 1879 & 0.7958 & 1.6512 & 0.6110 & 1.2329 & -0.3331 & 42.2597 & 1.9877 & 0.2177 \\
\hline 5 & 2392 & 0.8282 & 1.7844 & 0.7360 & 1.3867 & -0.2803 & 43.9837 & 1.9098 & 0.2685 \\
\hline 6 & 2926 & 0.8587 & 1.9510 & 0.8923 & 1.5719 & -0.2290 & 45.6001 & 1.8421 & 0.3169 \\
\hline 7 & 3477 & 0.8854 & 2.1509 & 1.0799 & 1.7868 & -0.1824 & 47.0179 & 1.7866 & 0.3625 \\
\hline 8 & 4044 & 0.9077 & 2.3830 & 1.2976 & 2.0295 & -0.1424 & 48.2042 & 1.7426 & 0.4049 \\
\hline 9 & 4624 & 0.9258 & 2.6454 & 1.5438 & 2.2979 & -0.1092 & 49.1658 & 1.7085 & 0.4436 \\
\hline 10 & 5214 & 0.9402 & 2.9356 & 1.8161 & 2.5896 & -0.0823 & 49.9301 & 1.6824 & 0.4785 \\
\hline 11 & 5812 & 0.9515 & 3.2506 & 2.1117 & 2.9020 & -0.0609 & 50.5309 & 1.6624 & 0.5091 \\
\hline 12 & 6416 & 0.9604 & 3.5872 & 2.4275 & 3.2324 & -0.0440 & 51.0011 & 1.6470 & 0.5351 \\
\hline 13 & 7026 & 0.9673 & 3.9420 & 2.7604 & 3.5777 & -0.0306 & 51.3691 & 1.6352 & 0.5565 \\
\hline 14 & 7639 & 0.9727 & 4.3114 & 3.1070 & 3.9349 & -0.0201 & 51.6580 & 1.6261 & 0.5728 \\
\hline 15 & 8255 & 0.9770 & 4.6917 & 3.4638 & 4.3009 & -0.0117 & 51.8860 & 1.6189 & 0.5841 \\
\hline 16 & 8874 & 0.9804 & 5.0793 & 3.8275 & 4.6725 & -0.0050 & 52.0669 & 1.6133 & 0.5903 \\
\hline
\end{tabular}




\begin{tabular}{|c|c|c|c|c|c|c|c|c|c|}
\hline time (ms) & turn & $\beta$ & $\gamma$ & $E_{k}(\mathrm{GeV})$ & $P(\mathrm{GeV} / \mathrm{c})$ & $\eta$ & $f_{\mathrm{rf}}(\mathrm{MHz})$ & $T_{0}(\mu \mathrm{s})$ & $\Delta E_{k}(\mathrm{MeV})$ \\
\hline 17 & 9497 & 0.9831 & 5.4704 & 4.1944 & 5.0462 & 0.0003 & 52.2114 & 1.6088 & 0.5911 \\
\hline 18 & 10119 & 0.9853 & 5.8613 & 4.5612 & 5.4188 & 0.0046 & 52.3276 & 1.6053 & 0.5868 \\
\hline 19 & 10742 & 0.9871 & 6.2483 & 4.9243 & 5.7870 & 0.0081 & 52.4217 & 1.6024 & 0.5772 \\
\hline 20 & 11367 & 0.9886 & 6.6279 & 5.2805 & 6.1475 & 0.0110 & 52.4983 & 1.6001 & 0.5625 \\
\hline 21 & 11992 & 0.9897 & 6.9965 & 5.6263 & 6.4972 & 0.0133 & 52.5610 & 1.5981 & 0.5428 \\
\hline 22 & 12618 & 0.9907 & 7.3507 & 5.9587 & 6.8328 & 0.0152 & 52.6125 & 1.5966 & 0.5183 \\
\hline 23 & 13245 & 0.9915 & 7.6874 & 6.2746 & 7.1515 & 0.0168 & 52.6550 & 1.5953 & 0.4892 \\
\hline 24 & 13872 & 0.9922 & 8.0033 & 6.5710 & 7.4504 & 0.0181 & 52.6901 & 1.5942 & 0.4557 \\
\hline 25 & 14499 & 0.9927 & 8.2957 & 6.8454 & 7.7269 & 0.0192 & 52.7190 & 1.5934 & 0.4182 \\
\hline 26 & 15127 & 0.9932 & 8.5620 & 7.0952 & 7.9784 & 0.0201 & 52.7428 & 1.5926 & 0.3770 \\
\hline 27 & 15755 & 0.9935 & 8.7995 & 7.3181 & 8.2029 & 0.0208 & 52.7622 & 1.5920 & 0.3324 \\
\hline 28 & 16383 & 0.9938 & 9.0064 & 7.5121 & 8.3982 & 0.0214 & 52.7779 & 1.5916 & 0.2849 \\
\hline 29 & 17011 & 0.9940 & 9.1806 & 7.6756 & 8.5626 & 0.0219 & 52.7903 & 1.5912 & 0.2349 \\
\hline 30 & 17640 & 0.9942 & 9.3206 & 7.8069 & 8.6947 & 0.0222 & 52.7997 & 1.5909 & 0.1828 \\
\hline 31 & 18268 & 0.9944 & 9.4251 & 7.9050 & 8.7933 & 0.0225 & 52.8065 & 1.5907 & 0.1290 \\
\hline 32 & 18897 & 0.9944 & 9.4932 & 7.9689 & 8.8576 & 0.0226 & 52.8108 & 1.5906 & 0.0741 \\
\hline 33 & 19525 & 0.9945 & 9.5243 & 7.9981 & 8.8869 & 0.0227 & 52.8127 & 1.5905 & 0.0186 \\
\hline
\end{tabular}




\section{Bibliography}

[1] S. Y. Lee, "Accelerator Physics", second edition (World Scientific, Singapore, 2004).

[2] Booster staff and E. L. Hubbard, ed, "Booster Synchrotron", Fermilab report TM-405, 1973.

[3] Bruce Worthel, "Booster rookie book", Fermilab internal report, 1998.

[4] E. Prebys, C. Ankenbrandt, W. Chou, A. Drozhdin, P. Kasper, J. Lackey, N. Mokhov, W. Pellico, R. Tomlin and R. Webber, "Increasing the intensity of the Fermilab Booster", PAC 2003, Portland, Oregon.

[5] A. Drozhdin, The Booster lattice model in MAD file. It can be found at http://lattices.fnal.gov/aid_repository/fileinfo.php?lattices/booster/official/ design/nbooster.lat.

[6] X. Huang, S. Ohnuma, "Compensation of dogleg effect in Fermilab booster", Fermilab Technical Memo TM-2225.

[7] J. Amundson, J. Lackey, P. Spentzouris, G. Jungman and L. Spentzouris, "Calibration of the Fermilab Booster ionization profile monitor", Phys. Rev. ST Accel. Beams 6, 102801 (2003) 
[8] J. Safranek, "Experimental Determination of Storage Ring Optics Using Orbit Response Measurements", Nucl. Instr and Meth. A, 388, 27 (1997).

[9] H. Grote and F.C. Iselin, The MAD Program, Version 8.1, User's Reference Manual, CERN/SL/90-13(AP) (1991).

[10] M. Borland, "Users Manual for elegant", available online at http://www.aps.anl.gov/asd/oag/manuals/ elegant_ver14.1/elegant.html

[11] Applications of the ORM method for NSLS, ALS, PEP-II, APS, etc. are reported.

[12] J. Nocedal and S. J. Wright, "Numerical Optimization", (Springer, 1999)

[13] W. H. Press, S. A. Teukolsky, W. T. Vetterling, B. P. Flannery, "Numerical Recipes in C - The Art of Scientific Computing", second edition, (Cambridge University Press, 1992)

[14] S. Shukla, a console program to take orbit response data for the Booster developed in 1997.

[15] Jim Lackey, private communications.

[16] J. Irwin, C.X. Wang, and Y.T. Yan, "Model-Independent Beam Dynamics Analysis", Phys. Rev. Lett. 82, 1684 (1999); Chun-xi Wang, Ph.D. thesis, unpublished (Stanford University, 1999).

[17] Chun-xi Wang, Vadim Sajaev, and Chih-Yuan Yao, "Phase advance and $\beta$ function measurements using model-independent analysis", Phys. Rev. ST Accel. Beams 6, 104001 (2003).

[18] Y.T. Yan, Y. Cai, F-J. Decker, J. Irwin, J. Seeman, S. Ecklund, M. Sullivan, J. Turner, U. Wienands, "PEP-II Beta Beat Fixes with MIA", SLAC-PUB-10369 (2003). 
[19] X. Huang, S. Y. Lee, E. Prebys, and R. Tomlin, "Application of Independent Component Analysis for Beam Diagnosis", Proc. PAC05, Knoxville, TN (2005)

[20] X. Huang, S. Y. Lee, E. Prebys, and R. Tomlin, "Application of independent component analysis to Fermilab Booster", Phys. Rev. ST Accel. Beams 8, 064001 $(2005)$

[21] Aapo Hyvarinen, Juha Karhunen, Erkki Oja, "Independent Component Analysis", (John Wiley \& Sons, New York, 2001)

[22] S. Choi, A. Cichocki and Y. Denville, "Differential decorrelation for nonstationary source separation", in Proc. of ICA, pp. 319-322, (San Diego, California, December 9-13, 2001).

[23] J-F. Cardoso, "The three easy routes to independent component analysis; contrast and geometry", in Proc. of the ICA 2001 workshop, San Diego, Dec. 2001.

[24] D. T. Pham, P. Garat, and C. Jutten, "Separation of a mixture of independent sources through a maximum likelihood approach", in Signal Processing VI, Theories and Applications, 771-774, Brussels, Belgium, Aug. 1992.

[25] L. Tong, R. Liu, V.C. Soon, Y.F. Huang, "Indeterminacy and identifiability of blind identification", IEEE Trans Circuits Syst I 38(5), 499-509, ( 1991)

[26] A. Belouchrani, K. Abed-Merain, J.F. Cardoso, and E. Moulines, "A blind source separation technique using second order statistics", IEEE Trans. Signal Processing, 45, 434-444, (1997)

[27] D. T. Pham and J-F. Cardoso, "Blind separation of instantaneous mixtures of non-stationary sources", IEEE Trans. Signal Processing, 49, 1837-1848, (2001) 
[28] J. F. Cardoso and A. Souloumiac, "Jacobi angles for simultaneous diagonalization", SIAM J. Mat. Anal. Appl., 17, 161, (1996).

[29] S. Choi, A. Cichocki, A Belouchrani, "Blind Separation of Second-order Nonstationary and Temporally Colored Sources", Journal of VLSI Signal Processing, 32, 93 (2002).

[30] A. Belouchrani and A. Cichocki, "Robust whitening procedure in blind source separation context", Electronics Letters, 36, 2050-2051, Nov. 2000.

[31] R. Bartolini, A. Bazzani, M. Giovannoizzi, W. Scandale and E. Todesco, "Tune Evaluation in Simulations and Experiments", CERN SL/95-84 (1984).

[32] J. F. Cardoso and A. Souloumiac, "Blind beam forming for non Gaussian signals", IEE Proceedings-F, 140(6), 362-370, 1993.

[33] A. Hyvarinen and E. Oja, "A fast fixed-point algorithm for independent component analysis", Neural Computation, 9(7), 1483-1492, 1997

[34] M. Ellison et al., "Experimental measurements of Invariant surface near a 2D nonlinear resonance", Phys. Rev. E50, 4051 (1994); S.Y. Lee et al., Phys. Rev. Lett., 67, 3768 (1991); D.D. Caussyn et al., Phys. Rev. A46, 7942 (1992); M. Syphers, et al., Phys. Rev. Lett. 71, 719 (1993); D. Li et al., Phys. Rev. E48, R1638 (1993); H. Huang et al., Phys. Rev. E48, 4678 (1993); Y. Wang, et al., Phys. Rev. E49, 1610 (1994); S.Y. Lee, Phys. Rev. E49, 5706 (1994).

[35] D.A. Edwards and L.C. Teng, "Parametrization of linear coupled motion in periodic systems", IEEE Trans. Nucl. Sci. 20(3), 885-889 (1973).

[36] J. Laskar, "Frequency analysis for multi-dimensional systems, Global dynamics and diffusion", Physica D,67,257 (1993) 
[37] Jim Lackey, private communications.

[38] R. Tomlin, W. Chou, F. Ostiguy, "Booster Chromaticity Measurement", Nov. 11, 2003, unpublished.

[39] X. Yang, J. MacLachlan, "Booster Synchrotron Frequency Below Transition", Fermilab Technical Memo TM-2239

[40] X. Yang, J. MacLachlan, "Energy Loss Estimates at Several Beam Intensities in the Fermilab Booster", Fermilab Technical Memo TM-2244

[41] K. Y. Ng, "Coupling impedance of laminated magnets", Fermilab Physics Note FN-0744.

[42] K. Y. Ng, "Betatron tune shifts and Laslett image coefficients", Fermilab Technical Memo TM-2152.

[43] Alex W. Chao, "Physics of collective beam instabilities in high energy accelerators", (John Wiley \& Sons, Inc, 1993, New York)

[44] X. Huang, S.Y. Lee, Eric Prebys, Chuck Ankenbrandt, "Fitting the Fully Coupled ORM for the Fermilab Booster", Proc. PAC05, Knoxville, TN (2005).

[45] Code from James Amundson was used to fit the profile data and calibrate the beam width.

[46] S. Ohnuma, "Elliptic distribution in longitudinal phase space", Fermilab Technical Memo TM-1381-A, 1986

[47] See e.g. A. Piwinski, "Intrabeam Scattering", CERN-92-01, p. 226 (1992).

[48] M. Reiser, "Theory and design of charged particle beams", (Wiley-Interscience, 1994) 
[49] S.Y. Lee and J.M. Wang, Proceedings of the Particle Accelerator Conference 1985, p. 2323 (1985).

[50] A. Sorensen, Particle Accelerators 6, 141 (1975).

[51] M.H. Wang, and S.Y. Lee, "RF Voltage Modulation and Coupled Bunch Instabilities", Journal of Applied Physics, 92, 555 (2002). 


\section{Xiaobiao Huang}

\section{Education}

Indiana University, Bloomington, 8/2001 - 9/2005, Ph.D. in Physics

Tsinghua University, Beijing, 9/1994 - 7/1999, B.S. in Modern Applied Physics,

B.E. in Computer Technology and Application

\section{Honors and Awards}

IUB Physics Departmental Award (2004), passed PhD qualifying exam at IUB

Physics Department with distinction (2002), Excellent Graduate of Tsinghua

University (1998), Ye Qisun Scholarship (1998), University-level Excellence

Scholarship (1997), Dongshi Dongfang Scholarship (1996), Excellent Student of

Grade 94 Scholarship (1995), Excellent freshman of Grade 94 Scholarship (1994)

\section{Work \& Research Experiences}

- 3/2003 - Present, Fermi National Accelerator Laboratory, Thesis work on Beam Diagnosis and Lattice Modeling of the Fermilab Booster

- 7/1999 - 6/2001, Zhongxing Telecom Co., Shenzhen, China, Hardware R\&D Engineer

- 9/1998 - 7/1999, Information Laboratory, Department of Computer Science \& Technology, Tsinghua University, Thesis work on Human Face Locating and Tracking Based on Stereo Vision

- 1/1998 - 7/1998, Institute of Physics, Chinese Academy of Science, Thesis work on Experimental Research on Photo-refractive Effect of Semi-insulating Multiple Quantum Wells 